\title{
Optical and Near Infrared studies of the photometric structure and starburst activity of Blue Compact Dwarf Galaxies
}

\author{
Dissertation \\ zur Erlangung des Doktorgrades \\ der Mathematisch-Naturwissenschaftlichen Fakultäten \\ der Georg-August-Universität zu Göttingen
}

vorgelegt von

Kai Gerhard Noeske

aus

Darmstadt

Göttingen 2003 
D 7

Referent: Prof. Dr. Klaus Fricke

Korreferentin: Priv. Doz. Dr. Uta Fritze-von Alvensleben

Tag der mündlichen Prüfung: 31.3.2003 


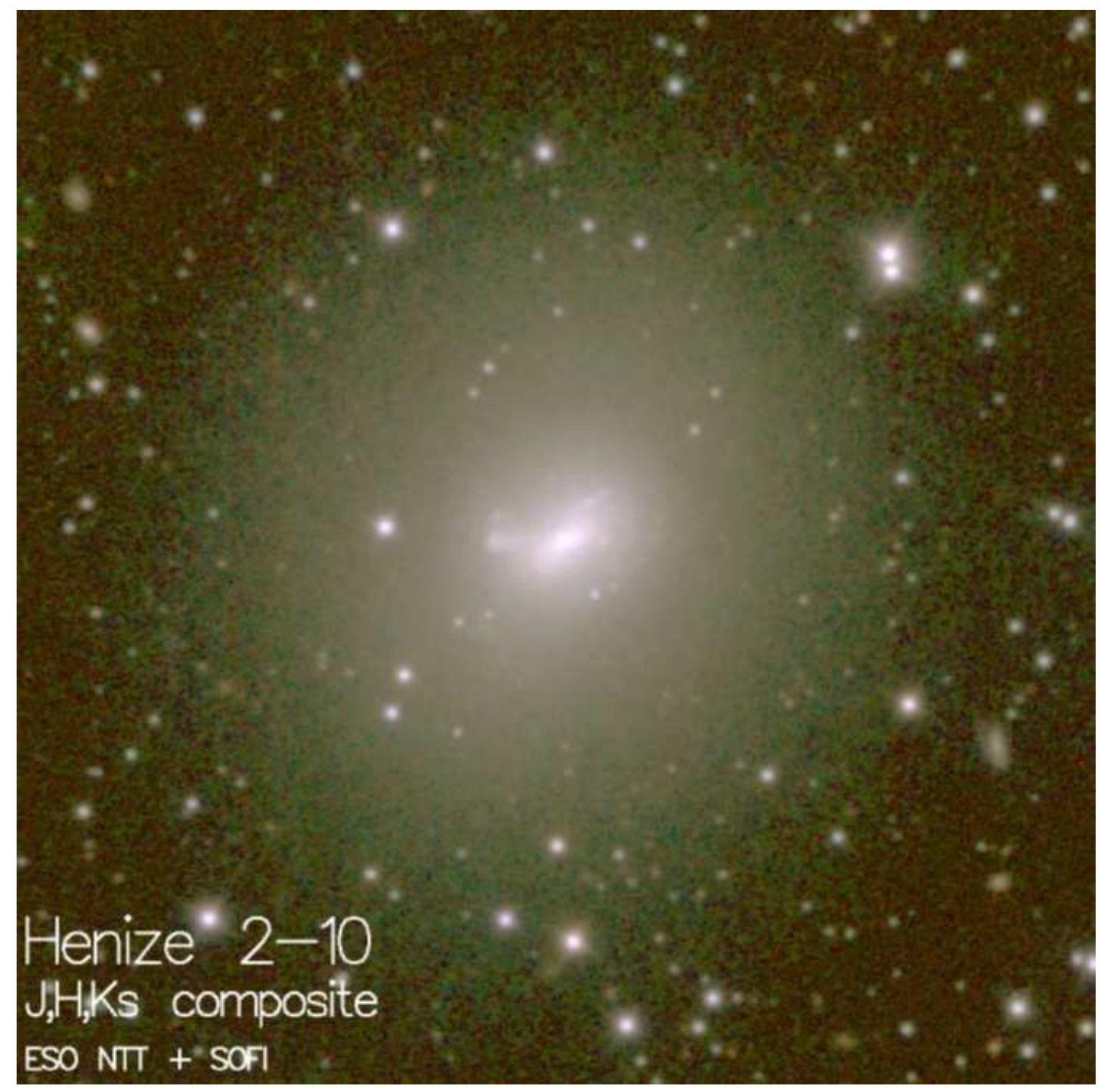

Für Polis und meine Eltern 



\section{Danksagung}

Zuerst möchte ich Prof. Dr. Klaus Fricke herzlich danken, der mir diese Arbeit ermöglicht und mich als mein Doktorvater betreut und auf vielfältige Weise unterstützt hat.

Besonders danke ich Dr. Polychronis Papaderos für alles das, was er in den vergangenen 6 Jahren für mich getan und mich gelehrt hat. Vor allem danke ich ihm für die wertvollen Gespräche, für seine aufbauenden Worte und hilfreiche Kritik, und für seine Hilfe und Freundschaft.

Herzlich bedanken möchte ich mich auch bei Dr. Luz Marina Cairós für ihre Zusammenarbeit, ihre Hilfe, und für ihre Gelassenheit, die in arbeitsreichen Zeiten immer sehr hilfreich war.

Dr. Uta Fritze-v. Alvensleben danke ich herzlich für die freundliche Übernahme der Korreferats dieser Arbeit und viele lehrreiche Gespräche. Ihr und ihrer Arbeitsgruppe möchte ich außerdem für die freundliche Bereitstellung und Hilfe bei der Anwendung des GALEV-Evolutionssyntheseprogramms danken.

Dr. Yuri Izotov und Dr. Natalya Guseva danke ich für die freundliche und lehrreiche Zusammenarbeit während ihrer zahlreichen Aufenthalte in Göttingen. Dankbar bin ich auch Dr. José Vílchez, Dr. Rafael Guzmán, Dr. Trinh X. Thuan, Dr. Regina SchulteLadbeck, Dr. Jorge Iglesias-Páramo und Dr. Armando Gil de Paz für ihre Gastfreundschaft.

Ingo Berentzen, Peter Weilbacher und Jens Bicker danke ich für ihren unentbehrlichen Einsatz bei der Betreuung der Rechner.

Meinen Zimmerkollegen Peter Anders, Jens Bicker, Thomas Lilly und Jochen Schulz danke ich für ihre nette Gesellschaft, die dafür gesorgt hat, daß ich mich buchstäblich zu Hause fühlen konnte. Ebenfalls danken möchte ich den Mitarbeiterinnen und Mitarbeitern der Sternwarte, die zu einer freundlichen Atmosphäre beigetragen haben.

Besonders lieben Dank schulde ich Katja Hosang für ihre Geduld und ihr liebevolles Verständnis, und dafür, daß sie mich mit jeder ihrer Sommersprossen immer wieder daran erinnert, wie schön die Sterne wirklich funkeln können.

Insbesondere möchte ich meinen Eltern Olaf und Isolde Noeske danken, die mir mein Studium ermöglicht und mir immer zur Seite gestanden haben, und meinen Geschwistern Christiane und Jens, die immer für mich da waren. 



\section{Contents}

\section{A Introduction}

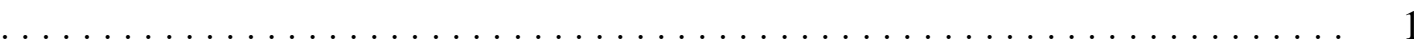

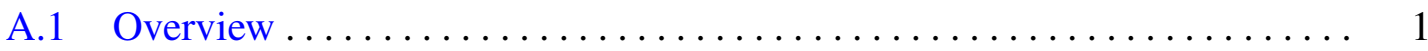

A.2 Blue Compact Dwarf galaxies (BCDs): classification criteria and

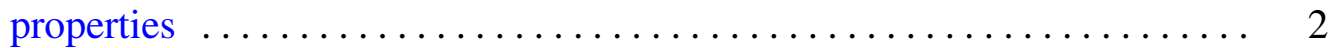

A.2.1 Relevance of BCDs to the understanding of dwarf galaxy evolution 3

A.2.2 Extremely metal-deficient BCDs ................. 4

A.3 Morphological classification of BCDs; "cometary" objects ......... 4

A.4 The stellar low surface brightness host galaxy $\ldots \ldots \ldots \ldots \ldots \ldots \ldots .5$

A.4.1 Dynamical importance of the stellar LSB host and scenarios of

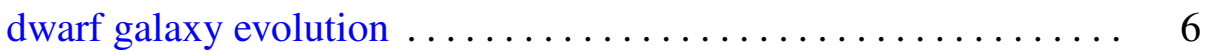

A.4.2 Relations between the stellar LSB host and star-forming activity in dwarf galaxies . . . . . . . . . . . . . . 7

A.4.3 Motivation of Near Infrared Observations ................ 7

A.5 Environmental influences on the star-forming activity and evolution of

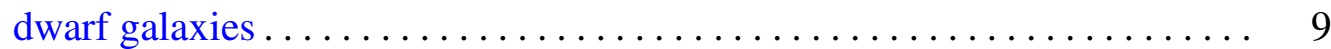

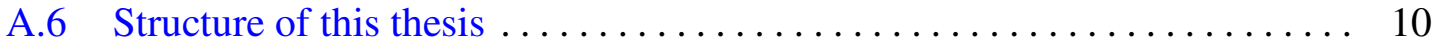

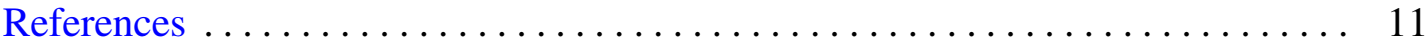

B Summary of the individual research projects and results

B.1 Near Infrared studies of the stellar host galaxy and starburst activity in

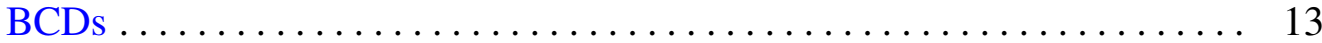

B.1.1 New insights into the structure of the stellar host galaxy $\ldots \ldots \ldots 13$

B.1.2 Relations between the stellar LSB host and star-forming activity .. 14

B.2 The relation of the "cometary" morphological class of BCDs to typical

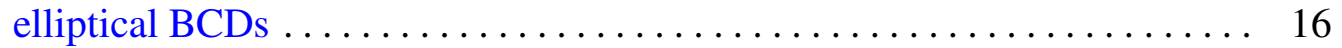

B.3 Environmental studies of star-forming dwarf galaxies . . . . . . . . . . 17

References .............................................. 19

C Scientific impact of this work and outlook on future research

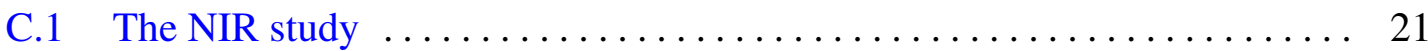


C.2 The relations between the stellar host galaxy and starburst activity . . . . 21

C.3 The question of Dark Matter in BCDs .................... 22

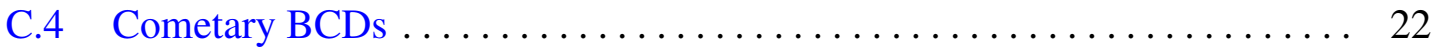

C.5 Faint companions in the close environment of star-forming dwarf galaxies 23

I New insights to the photometric structure of Blue Compact Dwarf Galaxies from deep Near-Infrared studies: I. Observations, surface photometry and decomposition of surface brightness profiles

K.G. Noeske, P. Papaderos, L.M. Cairós, K.J. Fricke . . . . . . . . . . . . 25

I.1 Introduction. ................................... 26

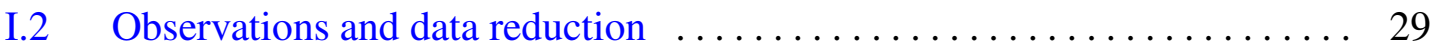

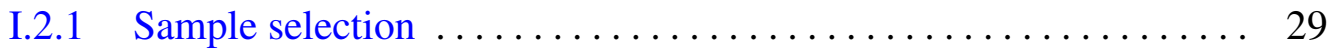

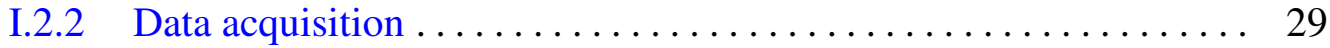

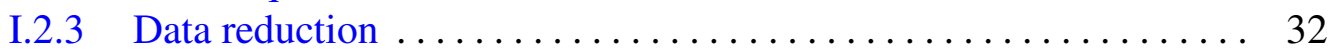

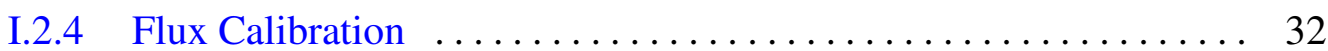

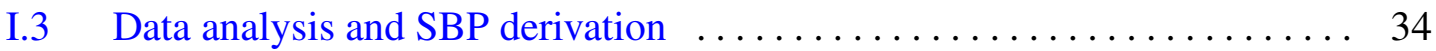

I.3.1 Derivation of surface brightness and color profiles ......... 34

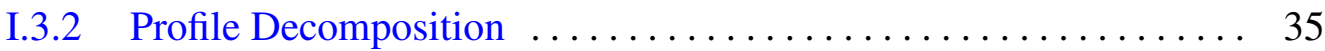

I.3.3 Aperture photometry and total magnitudes .............. 41

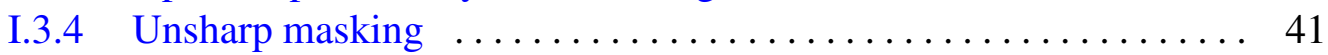

I.3.5 Colors of the underlying LSB host galaxy $\ldots \ldots \ldots \ldots \ldots \ldots \ldots 4$

I.4 Results and discussion of individual objects $\ldots \ldots \ldots \ldots \ldots \ldots \ldots \ldots . \ldots \ldots$

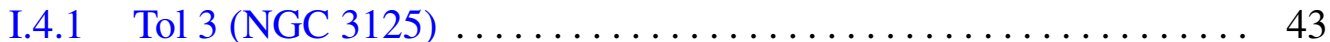

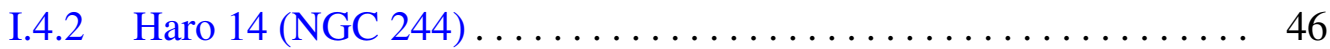

I.4.3 UM $461 \ldots \ldots \ldots \ldots \ldots \ldots \ldots \ldots \ldots \ldots \ldots \ldots \ldots \ldots \ldots \ldots$

I.4.4 Henize $2-10($ ESO $495-G 21) \ldots \ldots \ldots \ldots \ldots \ldots \ldots \ldots \ldots \ldots . . \ldots 49$

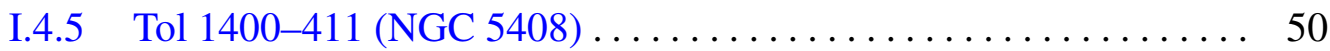

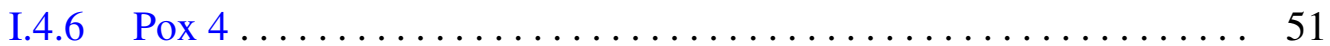

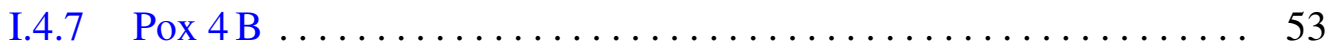

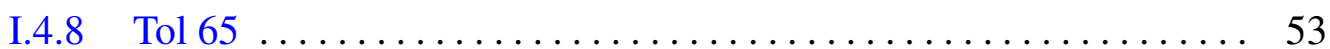

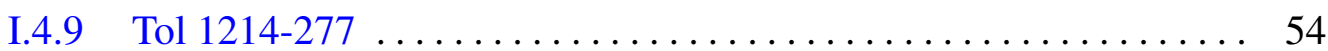

I.4.10 Mkn 178 (UGC 6541) . . . . . . . . . . . . . . . . . . 56

I.4.11 Mkn 1329 (IC 3589/91, UGC 7790, VCC 1699) . . . . . . . . . . . 57

I.4.12 IC4662 (ESO 102-G014, He 2-269) .................. 58

I.4.13 UM 488 (Mkn 1304, SBS 1139+006, UGC 6665) . . . . . . . . . . 60

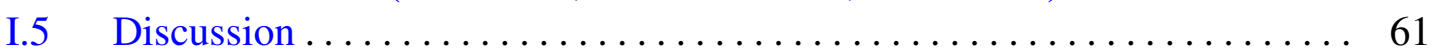

I.5.1 The shape of NIR surface brightness profiles $\ldots \ldots \ldots \ldots \ldots \ldots 61$

I.5.2 Hints to a centrally flattening exponential distribution in the underlying stellar LSB component? ............... 62

I.5.3 Fitting the LSB component $\ldots \ldots \ldots \ldots \ldots \ldots \ldots \ldots \ldots \ldots \ldots$

I.5.4 The colors of the stellar LSB host galaxy $\ldots \ldots \ldots \ldots \ldots \ldots . \ldots 6$

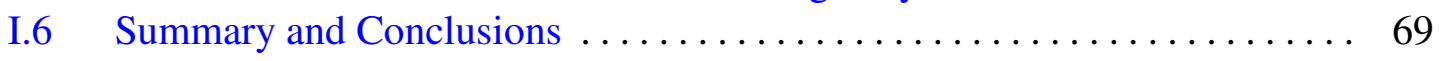

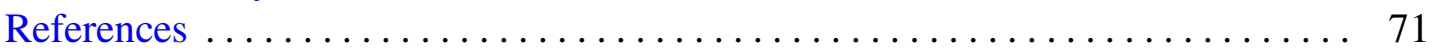


II New insights to the photometric structure of Blue Compact Dwarf Galaxies from deep Near-Infrared studies: II. The second sample of northern BCDs

K.G. Noeske, P. Papaderos, L.M. Cairós, K.J. Fricke ... . . . . . . . . . . 75

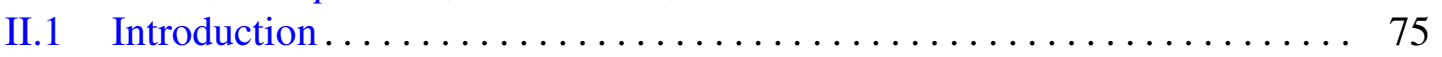

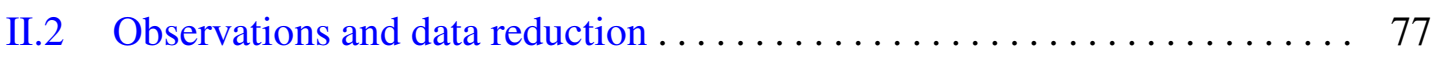

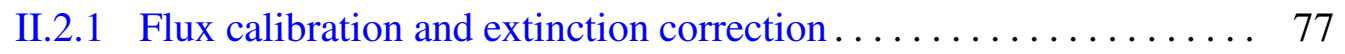

II.3 Surface photometry and profile decomposition $\ldots \ldots \ldots \ldots \ldots \ldots \ldots \ldots$

II.3.1 Colors of the underlying LSB host galaxy $\ldots \ldots \ldots \ldots \ldots \ldots . \ldots 1$

II.3.2 Unsharp masking technique \& aperture photometry . . . . . . . 84

II.4 Results and discussion of individual objects $\ldots \ldots \ldots \ldots \ldots \ldots \ldots \ldots$

II.4.1 Mkn 314 (NGC 7468, UGC 12329) . ................ 84

II.4.2 Mkn 209 (UGCA 281, I Zw 36, Haro 29) . . . . . . . . . . . . . 86

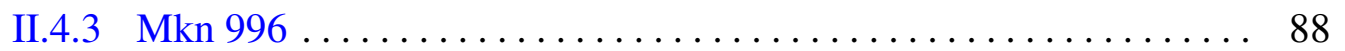

II.4.4 Mkn 370 (NGC 1036, UGC 02160) . . . . . . . . . . . . . 89

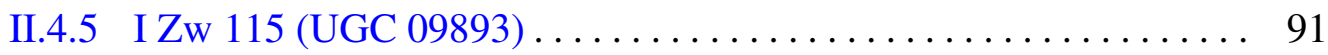

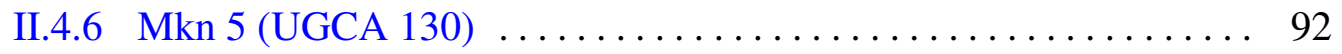

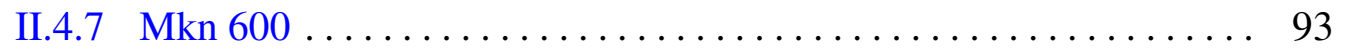

II.4.8 NGC $6789($ UGC 11425$) \ldots \ldots \ldots \ldots \ldots \ldots \ldots \ldots \ldots \ldots . \ldots \ldots . \ldots \ldots$

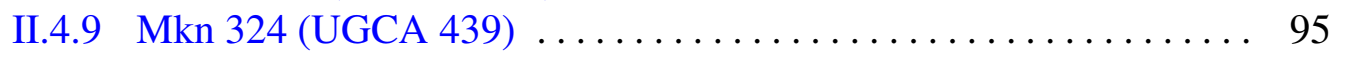

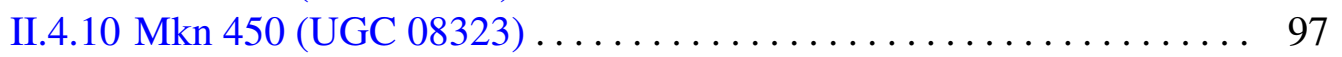

II.4.11 NGC 5058 (UGC 08345, MKN 786) . . . . . . . . . . . . . 98

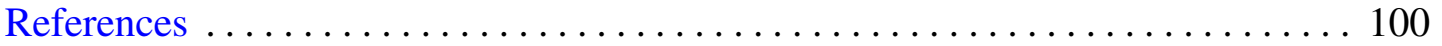

III New insights to the photometric structure of Blue Compact Dwarf Galaxies from deep Near-Infrared studies: III. The photometric structure of the stellar host galaxy and its relations to star-forming activity

K.G. Noeske, P. Papaderos, L.M. Cairós, K.J. Fricke . . . . . . . . . . . . . . 103

III.1 Introduction . . . . . . . . . . . . . . . . . . . . . . . . . . . . . . . 104

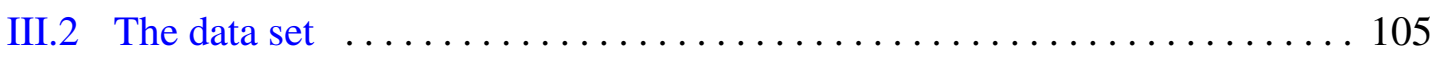

III.3 The photometric structure of the LSB host of BCDs . . . . . . . . . . 107

III.3.1 Central surface brightness and exponential scale length . . . . . . 107

III.3.2 The dependence of the structural properties of the LSB host on its

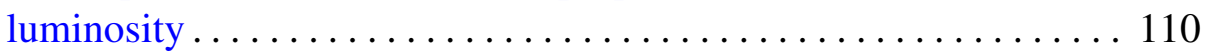

III.4 Inwards-flattening exponential (type $\mathrm{V}$ ) $\mathrm{SBP}_{\mathrm{LSB}} \ldots \ldots \ldots \ldots \ldots \ldots \ldots \ldots \ldots$

III.4.1 Frequency of type V SBP $\mathrm{LSB}_{\mathrm{L}}$ and relation to the BCD morphology 112

III.5 Relations between the structure of the LSB host and the properties of the

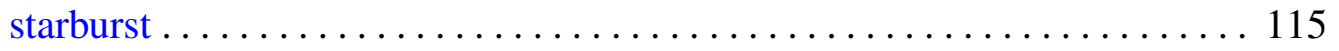

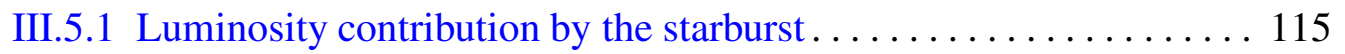

III.5.2 A tentative link between the structure of the stellar host and the occurrence and extent of burst-like star formation in typical BCDs . 117

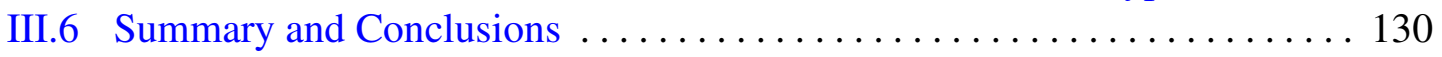

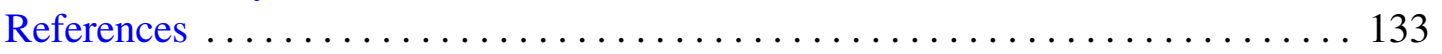


IV The Cometary Blue Compact Dwarf Galaxies Mkn 59 and Mkn 71:A Clue to Dwarf Galaxy Evolution?

K.G. Noeske, N.G. Guseva, K.J. Fricke, Y.I. Izotov, P. Papaderos, T.X. Thuan . . . 135

IV.1 Introduction. . . . . . . . . . . . . . . . . . . . . . . . . . . . . . 136

IV.2 Data acquisition and processing . . . . . . . . . . . . . . . 139

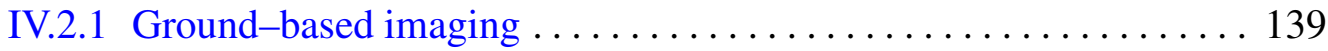

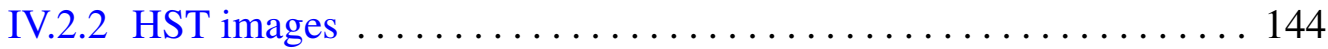

IV.2.3 Spectroscopic observations and data reduction $\ldots \ldots \ldots \ldots \ldots 146$

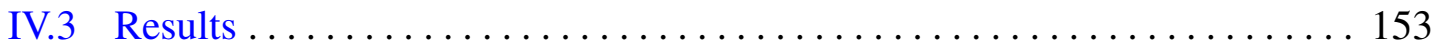

IV.3.1 Structural properties of the host galaxies $\ldots \ldots \ldots \ldots \ldots \ldots \ldots 153$

IV.3.2 Heavy element and line intensity distributions . . . . . . . . 155

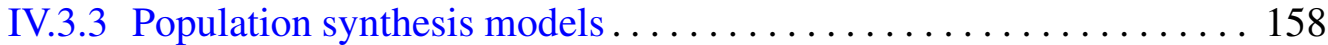

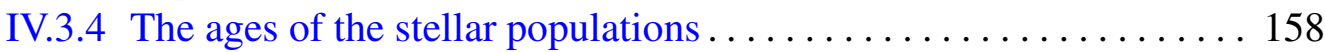

IV.4 Discussion . . . . . . . . . . . . . . . . . . . . . . . . . 163

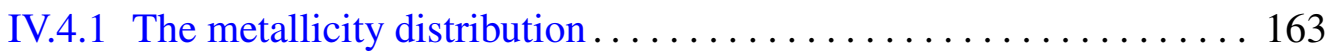

IV.4.2 Morphology vs. structural properties and age . . . . . . . . . 164

IV.4.3 An elongated structure of high gas density? . . . . . . . . . 165

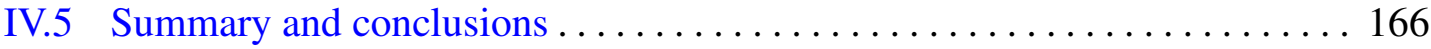

References ........................................ 168

V The Environment of Star-Forming Dwarf Galaxies: The Role Of Dwarf Companion Objects

K.G. Noeske,J. Iglesias-Páramo, J.M. Vílchez, K. J. Fricke . . . . . . . . . . . 173

V.1 Introduction.................................. 173

V.2 Sample Selection ................................. 174

V.2.1 The Long-Distance Sample . . . . . . . . . . . . . . . 175

V.3 Companion Search, Criteria for Isolation and Interaction . . . . . . . . 175

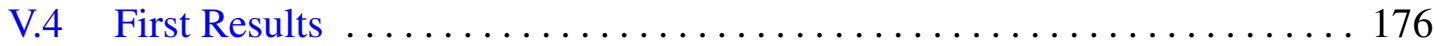

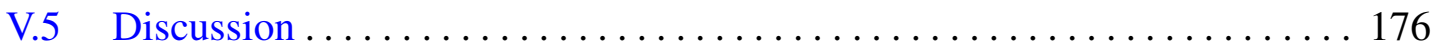

V.5.1 Environment and Bias Effects against Dwarf Companions . . . . . 176

V.5.2 Environmental Effects on Star Formation ................. . 178

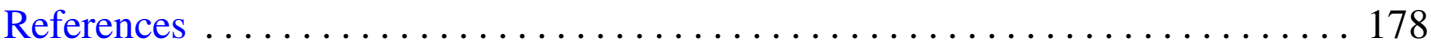

VI On faint companions in the close environment of star-forming dwarf galaxies: Possible external star formation triggers?

K.G. Noeske, J. Iglesias-Páramo, J.M. Vílchez, P. Papaderos, K.J. Fricke . . . . 181

VI.1 Introduction................................. 181

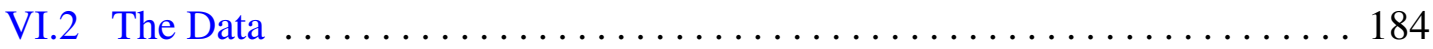

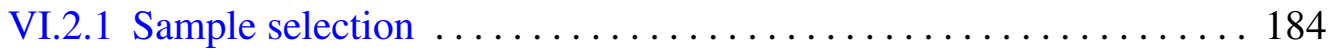

VI.2.2 Search for companion objects $\ldots \ldots \ldots \ldots \ldots \ldots \ldots \ldots \ldots \ldots \ldots$

VI.2.3 Distances and correction for Virgo Cluster infall . . . . . . . . . 188

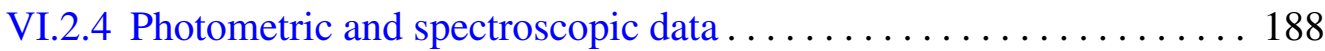

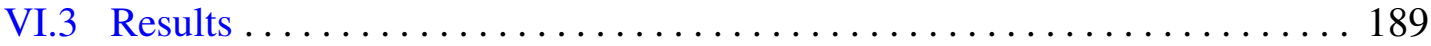

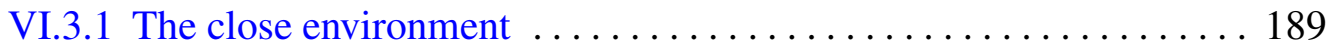

VI.3.2 Star Formation and Environment . . . . . . . . . . . . . . . . . . . . 192 
VI.4 Discussion .......................................... 194

VI.4.1 Frequency, distribution and properties of the companion candidates 194

VI.4.2 Close dwarf companions as starburst triggers? . . . . . . . . . . 195

VI.5 Summary and Conclusions .......................... 196

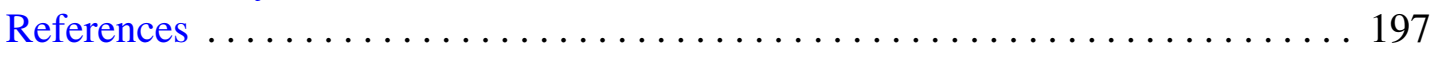





\section{Introduction}

\section{A.1 Overview}

The studies presented within this thesis aim at an improved understanding of the nature and starburst-driven evolution of Blue Compact Dwarf (BCD) Galaxies. These are gas-rich dwarf galaxies which display strong star-forming activity ("starbursts"). In view of current research on galaxy evolution, BCDs are considered important to the understanding of starburst activity, and of the starburst-driven evolution of dwarf and low-mass galaxies.

The investigations described in this work focus on the structure of the stellar components, the star-forming activity, and the environment of BCDs, as well as on the physical background of their morphological variety.

A major part of this thesis is dedicated to the study of the old stellar low-surface brightness (LSB) host galaxy of BCDs (Papers I-III). The structure of this component holds important information on the origin and impact of star-forming activity in dwarf galaxies, and on the dynamics and evolution of such systems. Previous studies of the stellar LSB host, carried out in visible wavelengths, were hampered by the dominant emission of the ongoing starburst. The present study analyses for the first time Near Infrared (NIR) images of a larger sample of BCDs, which are affected by the starburst emission to a lesser degree and therefore allow to study the stellar host with a better precision than in optical bands. The derived results provide new insights into the structure of the old and young stellar components in BCDs, and are discussed in view of proposed evolutionary scenarios of dwarf galaxies and the origin of starburst activity of BCDs.

A second study (Paper IV) focuses on the morphological variety of BCDs, the origin of which has not yet been systematically investigated. The dominant morphological class of BCDs in the local Universe displays an elliptical, evolved stellar host galaxy with centrally concentrated star-forming activity. Those objects are well-studied, at variance to the less frequent "cometary" $\mathrm{BCDs}$, in which strong star-forming activity takes place towards the end of an elongated stellar host galaxy. A first detailed investigation of two prototypical cometary BCDs is presented, and supplemented by literature data on other cometary objects. Cometary and elliptical BCDs are compared with respect to the structural properties and stellar ages of their host galaxies. The origin of the remarkable morphology of star-forming activity in cometary BCDs is discussed. The question is raised whether these objects may represent a distinct evolutionary stage of BCDs, or whether elliptical and cometary BCDs follow distinct evolutionary paths, determined by differing initial properties or different environments. 
Further attention is given to the study of environmental influences on the starforming activity and evolution of dwarf galaxies (Papers V and VI). BCDs reside at typically large distances to the next luminous neighbor galaxy, and do not appear to be significantly influenced by such distant luminous companions. Recent studies point, however, to the presence of numerous faint companion galaxies in the close environment of star-forming dwarf galaxies, the detection of which is frequently hindered by their low luminosities. As an alternative to gravitational perturbations by luminous galaxies, such faint companions at small distances have also been considered to significantly influence the star-forming activity of dwarf galaxies. This hypothesis is followed up in the present thesis, using a large sample of star-forming dwarf galaxies and improved galaxy catalogs to search for companions. Observational selection effects against faint companions are investigated. To moderate such selection effects, a sample of nearby star-forming dwarf galaxies is selected. Therefrom, the frequency of faint companions at close distances to the sample objects is constrained, and their possible influence on star-forming activity in dwarf galaxies is assessed.

The following introductory sections give an outline of the current state of knowledge on BCDs, and of their relevance to the research fields of dwarf galaxy evolution and starburst activity. Unsolved questions which motivated the present work are presented, as well as their relevance to actual research on the structure, dynamics, starforming activity and evolution of dwarf galaxies.

This introduction is intended to provide a summarizing overview. More detailed introductions into the topics followed up in this thesis are given in the individual papers (Sect. 1 of Papers I, IV \& VI). These Sections also contain more extensive literature reviews and references.

\section{A.2 Blue Compact Dwarf galaxies (BCDs): classification criteria and properties}

Dwarf Galaxies are extragalactic systems of low mass $\left(M \lesssim\right.$ few $\left.10^{9} M_{\odot}\right)$ and luminosity (absolute blue magnitude $>-18$ ), which form the most frequent galaxy class in the local Universe. In the local group of galaxies, where observational selection effects against such faint systems are comparably small, 36 dwarf galaxies are currently known, compared to only 3 luminous galaxies (see, e.g., Zwicky 1957, Binggeli \& Cameron 1991, van den Bergh et al. 2000).

Dwarf galaxies span a wide range of physical properties and morphologies, by which several subclasses are defined. Dwarf Ellipticals (dEs) or spheroidals (dSphs) are gas-poor objects which show an evolved, low-surface-brightness (LSB) stellar population with no appreciable signs of current star formation. Such dwarf galaxies are predominantly found in regions of high galaxy density, such as groups or clusters of galaxies. Gas-rich, star-forming dwarf galaxies, which populate on average regions of lower galaxy density, are classified into dwarf irregulars (dIs) and Blue Compact Dwarf Galaxies (BCDs). Both classes of objects have generally high gas mass fractions $(\sim 30 \%$, with individual fractions ranging from a few percent to $>90 \%$; see 
Thuan 1991 or Thuan \& Martin 1981). BCDs and dIs are mostly metal-poor, with heavy element mass fractions of the ionized gas ranging from 1/50 to $\sim 1 / 3$ of the solar value $\left(Z_{\odot}\right)$, and a mean value of the order of $1 / 10 Z_{\odot}$ (Kunth \& Sargent 1986). Within their stellar LSB host galaxies, dIs display a wide-spread star-forming activity at a low amplitude, with typical star formation rates of a few $10^{-3}$ - few $10^{-2}$ solar masses per year (e.g. Gallagher 1998), which in many cases may have proceeded at comparable intensities over a Hubble time (e.g. Hunter 1997). BCDs, on the other hand, undergo intense star-forming activity (few $10^{-2}->1$ solar masses per year, Thuan 1991), concentrated towards the center of an evolved stellar LSB host galaxy. This strong starforming activity in BCDs is generally believed to occur in short-lived events of $\lesssim 10^{7}$ yr, so-called starbursts, separated by longer quiescent episodes of several $10^{8}-10^{9} \mathrm{yr}$ (Thuan 1991, Elmegreen et al. 1996). Recent studies have alternatively suggested that BCDs may undergo prolonged episodes of moderately enhanced star-forming activity (e.g. Schulte-Ladbeck et al. 2001), rather than short-lived bursts.

To date, no standardized selection criteria have been defined for BCDs. Due to their star-forming activity, the spectra of BCDs are dominated by strong narrow emission lines and steep UV continua, similar to those of extragalactic H II regions. BCDs selected in spectroscopic surveys are therefore also referred to as "H II galaxies" (Salzer et al. 1989a, Terlevich et al. 1991, Telles \& Terlevich 1997). The samples by Thuan \& Martin (1981) include an additional morphological selection criterion, requiring a compact optical size $(<1 \mathrm{kpc})$ on photographic survey plates. In view of the nowadays available deeper images, this criterion describes a compact size of the high-surface brightness star-forming regions. Marlowe et al. (1999) have subsequently shown that BCDs, dwarf H II galaxies and morphologically selected "Blue Amorphous Galaxies" are likely to form a common physical class of objects, sharing a similar structure in their LSB host galaxies, chemical abundances of warm interstellar medium, and starforming activity.

\section{A.2.1 Relevance of BCDs to the understanding of dwarf galaxy evolution}

In the light of currently discussed theories of hierarchical cosmic structure formation, much importance is attached to dwarf galaxy research (e.g. Chiu et al. 2001, Dalcanton \& Craig 2001, van den Bosch \& Swaters 2001). Tests for such theories can be performed by means of a complete observational census of the dwarf galaxy population. Furthermore, elaborate studies of nearby dwarf galaxies may yield clues to processes operating in early pre-galactic low-mass building blocks at high redshift (see Sect. 1 of Paper I for references). However, to derive such constraints from present-day dwarf galaxies, it will be necessary to also understand the processes responsible for the variety of their observed properties.

For the above mentioned issues, BCDs are particularly interesting. Despite their ages of typically several Gyr, as demonstrated through the detection of an old stellar LSB host galaxy by Loose \& Thuan (1986), these systems are gas-rich and metaldeficient as described above, in resemblance to the properties assumed in unevolved galaxies in the early Universe. Their strong star-forming (SF) activity makes BCDs 
nearby testbeds to study the occurrence of starburst activity in low mass galaxies. Equally important is the possibility to explore how starburst events affect the spectrophotometric and chemodynamical evolution of such systems (see, e.g., Sect. A.4.2). Currently, the conditions and mechanisms that lead to starburst activity in dwarf galaxies remain a subject of debate. Various processes have been suggested, such as stochastic self-propagating star formation (Gerola et al. 1980), or the influence of a compact stellar component (Sect. A.4.2). Apart from such internal mechanisms, also interactions between a dwarf galaxy and its environment (Sect. A.5) are considered as possible triggering agents of starburst activity. A better understanding of such issues is essential for exploring the evolutionary pathways proposed to interrelate BCDs and other types of local dwarf galaxies, dEs and dIs, as well as their possible progenitors at higher redshift (see Sect. 1 of Paper I for details).

\section{A.2.2 Extremely metal-deficient BCDs}

Among the BCDs in the local Universe, a small fraction $(\lesssim 1 \%)$ of objects has been found to be extremely deficient of heavy elements, with an oxygen abundance $<1 / 20$ of the value measured in the solar neighbourhood (see Izotov \& Thuan 1999 and references therein). Such objects are of special cosmological interest. Their chemically unevolved state allows for close observational approaches, and narrow constraints, to the primordial element abundance ratios $\left(\mathrm{e} . \mathrm{g}{ }^{4} \mathrm{He} / \mathrm{H}\right)$, which can serve to derive constraints to cosmological parameters (Peimbert \& Torres-Peimbert 1974, Pagel et al. 1992, Izotov et al. 1997b).

It has been proposed that such extremely metal-poor BCDs may represent young galaxies in the local Universe, which started their star-forming activity less than $\sim 100 \mathrm{Myr}$ ago. This hypothesis was based on production timescales of heavy elements in evolving stellar populations, and on the absence of a conspicuous old stellar population in these galaxies, other than in the main class of less metal-rich BCDs (e.g. Izotov \& Thuan 1999, Fricke et al. 2001 and references therein). In spite of controversial discussions (e.g. Östlin 2000, Östlin \& Kunth 2001, Papaderos 1998, Papaderos et al. 2002, Hunt et al. 2003), no consensus has yet been reached about the evolutionary status of such young galaxy candidates. Nevertheless, these unevolved objects offer environments which hold clues to processes of galaxy formation and evolution in the early Universe.

\section{A.3 Morphological classification of BCDs; “cometary” objects}

Loose \& Thuan (1986) developed a classification scheme for BCDs which describes both the morphology of their star-forming regions and their stellar LSB host galaxy. A similar classification into objects with extended irregular and compact regular host galaxies was proposed by Telles et al. (1997). Also the morphology-and luminositybased classification for dwarf and luminous emission line galaxies by Salzer et al. (1989a) has been applied to star-forming dwarf galaxies. 
Loose \& Thuan (1986) found that $\sim 80-90 \%$ of their sample consists of $\mathrm{iE}-$ and nE-classified objects. Such BCDs show, respectively, an irregular (iE) or nuclear (nE) starburst component superposed on a smooth elliptical LSB host galaxy with optical colours pointing to ages of several Gyr (Loose \& Thuan 1986, Papaderos et al. 1996a, Telles \& Terlevich 1997, Cairós et al. 2001a, Bergvall \& Östlin 2002). In contrast, the few members of the rare "i0" class of BCDs examined so far have been found to be among the extremely metal-deficient young galaxy candidates (e.g. Izotov et al. 1997b, Papaderos et al. 2002) presented above, apparently lacking a smooth stellar underlying component that would have required previous episodes of SF to be built. The class of iI BCDs, characterized by both irregular LSB components and starburst regions, has not yet been studied systematically. Among those, the "iI,C" ("cometary") BCDs (Loose \& Thuan 1986) are particularly intriguing. The cometary appearance of these objects, which contribute about $10 \%$ of the local BCD population, is caused by a bright starforming complex at the one end of an elongated stellar body (Paper IV, Sect. 1). This morphology of the star-forming regions is in contrast to typical iE/nE BCDs, where starburst activity is centrally concentrated.

Irregular morphological types may show systematically lower metal abundances, larger velocity dispersions and higher excitation parameters of the ionized gas in their star-forming regions (Salzer et al. 1989b, Telles et al. 1997) than regular objects. Systematic comparisons of, e.g., the stellar host galaxies and neutral gas components for different morphological types have not yet been undertaken. The physical relations between various morphological types of star-bursting dwarf galaxies are therefore to date not understood. The various morphologies may, e.g., represent a large range of intrinsic physical parameters of the host galaxies, different triggering and propagation mechanisms of the starburst, or may reflect different evolutionary states (Paper IV, Sect. 1).

\section{A.4 The stellar low surface brightness host galaxy}

Despite much previous effort, the current knowledge on dwarf galaxy evolution is still too fragmentary to allow for a commonly accepted, unifying scenario. One cornerstone to advances in this field, as suggested by preceding work, is the investigation of the unresolved LSB host galaxy. This old stellar component is invariantly found in all local dwarf galaxies, except for the extremely rare i0 BCDs (Loose \& Thuan 1986). The LSB host dominates the surface brightness and color distribution in a BCD's outskirts, and accounts typically for $\sim 1 / 2$ of its total $B$ band luminosity (Papaderos et al. 1996b). The spectrophotometric properties of the old stellar populations form the fossile record of the history of star-forming activity of a dwarf galaxy, allowing to place, e.g., constraints on the system's formation age. Due to its higher mass-to-light ratio, the LSB host provides the bulk of the stellar mass of a BCD. Therefore, the determination of its structural properties and dynamics is crucial for at least two central topics of dwarf galaxy research: the evolutionary connections between different types of dwarf galaxies, and the regulation of their SF activities. 


\section{A.4.1 Dynamical importance of the stellar LSB host and scenarios of dwarf galaxy evolution}

Dwarf galaxies share a variety of basic physical parameters, such as similar masses, luminosities, optical extents and morphologies, and generally low metal abundances (see Sect. A.2). Therefore, as detailed in Sect. 1 of Paper I, various evolutionary scenarios have been suggested to account for the coexistence of gas-poor dEs/dSphs, gas-rich, moderately star-forming dIs and star-bursting BCDs. Such scenarios include, e.g., processes of gas infall and gas loss, different possibilities of the triggering of a starburst event, and either favor, or argue against, evolutionary transitions between different classes of dwarf galaxies (cf. Davies \& Phillipps 1988, Papaderos et al. 1996b, Meurer et al. 1998, Marlowe et al. 1999, and references therein).

Recent structural analyses revealed that BCDs, dIs and dEs do not simply differ by their gas content and star-forming activity, contrary to what had been assumed over the past two decades. Instead, typical elliptical BCDs show a systematically higher central concentration of their stellar mass than dIs and dEs. On average, the central surface brightnesses of their LSB hosts are brighter by $\sim 1.5 \mathrm{mag}$, and their exponential scale lengths are smaller by a factor of $\sim 2$, than those found for dIs and dEs (Papaderos et al. 1996b, Marlowe et al. 1999). In a similar way, the surface densities of the neutral gas component of BCDs are by a factor of $\sim 5$ higher than those of dIs (e.g., van Zee et al. 2001).

Evolutionary transitions between BCDs and other dwarf galaxies require therefore a redistribution of gaseous and stellar mass on global scales. First studies of the kinematics of the neutral gas in few compact dwarf galaxies (e.g. Meurer et al. 1998 and references therein) led to the assumption that Dark Matter dominates their dynamics on all spatial scales. Should that be the case, then a significant mass redistribution is not possible, regardless of the energy released by a starburst event. Hence, the hypothesis of Dark Matter dominance rules out evolutionary transitions between BCDs and dIs/dEs. Recent observations (see Sect. 1 of Paper I, or e.g. Gil de Paz et al. 2002) and various evidence of different nature (Papaderos 1998) suggest, however, that BCDs may frequently not be dominated by Dark Matter within their optical extent. Under this condition, significant changes of the stellar mass distribution are to be expected, as a reaction to changes of the gravitational potential on global scales. The latter may be introduced by processes which change the dynamical state of the gas component, e.g., a large-scale gas infall from the H I halo. Similarly, the energy input of young massive stars and supernovae during the starburst may heat and accelerate the gas component of a BCD, leading to a global expansion of the gaseous and stellar matter, and possibly transforming a compact BCD into a more diffuse dI. Either of the latter two scenarios has been presented and critically discussed in Papaderos et al. (1996b).

Evidently, precise information on the total mass of the stellar LSB host and its radial distribution is a prerequisite to modeling the total mass distribution and dynamics of BCDs, with the aim to distinguish between the latter dynamic scenario of dwarf galaxy evolution, and the former static one. The same information is also important as input for hydrodynamical models of starburst-driven outflows of metal-enriched gas. Such 
processes, referred to e.g. as "galactic winds" or "blowout", are considered important to the chemical evolution of dwarf galaxies, and were found to depend strongly on a galaxy's gravitational potential (e.g. De Young \& Heckman 1994, Mac Low \& Ferrara 1999, Silich \& Tenorio-Tagle 2001).

In either case, the radial distribution of the stellar LSB mass provides to date the best clue to the dynamics of the stellar mass in BCDs. Stellar kinematics of the LSB host have to date not been published, and would require high-resolution spectroscopy outside the star-forming regions, i.e. at very low surface brightnesses $\left(\mu_{B} \gtrsim 24.5 \mathrm{mag} / \operatorname{arcsec}^{2}\right)$.

\section{A.4.2 Relations between the stellar LSB host and star-forming activity in dwarf galaxies}

Apart from its importance to evolutionary scenarios, there is evidence that the stellar LSB component strongly influences star-forming activity in dwarf galaxies (Paper I, Sect. 1 \& Paper III, Sect. 5.2). Papaderos et al. 1996b reported that both the luminosity and the spatial extent of starburst activity in BCDs are closely related to the luminosity of the stellar host galaxy. The starburst strength of BCDs, as reflected on the soft X-ray emission observed by ROSAT, was found to increase towards higher central densities of the stellar LSB host (Papaderos 1998). For dIs, Hunter et al. (1998) found a close correlation between the surface densities of star formation activity and evolved stars.

Such lines of evidence strongly suggest that the stellar mass may significantly influence the gravitational potential within which the gas collapses to form stars. The influence of the stellar mass on the star-forming activity in dwarf galaxies provides therefore further support for the assumption that these objects are not Dark Matterdominated within their optical radius, as outlined in the previous subsection.

\section{A.4.3 Motivation of Near Infrared Observations}

To summarize, the exploration of dwarf galaxies requires further observational constraints to the structural properties and dynamics of their stellar LSB hosts. A precise determination of their structural and dynamic properties (e.g. radial surface brightness, mass density and color distribution, geometry) is a prerequisite to the understanding of their SF activity and evolution.

Information on the radial stellar mass density distribution of the stellar host is typically derived through surface photometry studies. Using various algorithms (for BCDs, see e.g. Papaderos et al. 1996a and references therein, Papaderos et al. 2002, or Paper I of the present thesis), a galaxy's two-dimensional intensity distribution is transformed into an azimuthally averaged, i.e. one-dimensional radial intensity distribution ("surface brightness profile"). From this intensity profile, the intrinsic radial luminosity density (and hence mass density, cf. Paper III, Sect. 5.2) distribution can be inferred by solving the equation of radiative transfer, with typically a number of simplifying assumptions.

For BCDs, such studies of the host galaxy are complicated by the emission from the centrally concentrated starburst. As mentioned above, the stellar host galaxy contains 
practically the entire stellar mass of a $\mathrm{BCD}$, as can be inferred from the high mass-tolight ratio of its old stellar populations. The young stellar populations, formed during current and recent episodes of star-forming activity, contribute on average one half of the $B$ band light of such systems, but comprise at most few percent of their stellar mass (cf., e.g., Krüger et al. 1995). Consequently, physically meaningful studies of $\mathrm{BCDs}$ require a decomposition of their total radial intensity profiles into those of either stellar component.

For this purpose, an empirical fitting function, typically an exponential, is applied to model the light distribution of the stellar host galaxy. A subtraction of this host galaxy model from the observed total intensity profile of the BCD yields, in addition, the radial light distribution of the starburst (see Paper I, Sect. 3.2).

Such an approach is, however, not easily performed. Especially at visible wavelengths, where the current knowledge on the LSB hosts of BCDs has been derived, the contribution of the starburst allows to constrain the LSB host model only at large galactocentric radii. In, e.g., the $B$ band, the starburst component contributes significantly to the observed emission down to a surface brightness level of $\gtrsim 24 \mathrm{~B}$ mag/arcsec ${ }^{2}$, or typically within the $\gtrsim 2$ inner exponential scale lengths of the host galaxy. Only at larger radii, where the starburst emission from young stars and extended ionized gas becomes negligible, the LSB host can be studied directly. However, to understand e.g. its influence on star-forming activity, it is essential to determine its intensity distribution also within small radii. This information is needed to constrain the gravitational potential of the stellar LSB component within the location of active star formation. As noted above, it is a common practice to extrapolate the model of the LSB host as obtained in its outer, unpolluted regions towards its center. However, there is observational evidence which suggests that this procedure is not always adequate. For some BCDs, the LSB hosts show a central deviation from the exponential slope typically derived in their outskirts (Papaderos et al. 1996a, Papaderos et al. 1999, Vennik et al. 2000, Fricke et al. 2001).

A promising way to alleviate the problem of the centrally concentrated, dominant starburst emission is to extend studies to the Near Infrared (NIR). At these wavelengths, the young stellar populations and the ionized gas contribute a smaller fraction to the total emission of the galaxy than in the optical (see Paper I, Sect. 1). The strength of this effect can be estimated using the models developed by Krüger et al. (1995) for starbursts in evolved dwarf galaxies. Assuming a stellar mass fraction of the burst of $\sim 1 \%$, typical for BCDs, the fractional contribution of the starburst to the integral light is $\gtrsim 4$ times lower in the $K$ band $(\lambda=2.2 \mu \mathrm{m})$ than in the $B$ band $(\lambda=0.4 \mu \mathrm{m})$. The models also predict that the fractional contribution of the ionized gas emission is $\sim 2-3$ times lower in $K$ than in $B$. In optical images, this extended ionized gas contributes an important fraction to a BCD's line-of-sight emission out to large radii (cf. e.g. Cairós et al. 2001b, Papaderos et al. 2002, Gil de Paz et al. 2002). The starburst emission becomes therefore negligible at smaller radii in the NIR than at optical wavelengths.

NIR images do not only allow to study the structural properties of a BCD's stellar LSB host towards smaller radii. In combination with optical data, they further allow to derive optical-NIR colors of the host galaxy (e.g. $B-J$ ). Such colors, corresponding 
to a long spectral baseline, allow for more precise constraints on the formation history of the stellar host than optical colors alone. (cf. Paper I, Sect. 1) .

A further benefit of combining optical and NIR observations comes from the wavelength dependence of dust extinction, which is e.g. in the $V$ band $(\lambda=0.55 \mu \mathrm{m})$ by a factor of $\approx 9$ larger than in $K$. Within the star-forming regions of BCDs, where irregularly distributed dust is known to be present (e.g. Cairós et al. 2001b, Lisenfeld et al. 2002), NIR data allow for the detection of regions obscured in the optical, and therefore provide a more complete view of the spatial occurrence of star formation. Recent results (e.g. Gil de Paz et al. 2000b) highlight the importance of such data for the general understanding of the starburst phenomenon in BCDs. In addition, combined optical-NIR intensity ratio maps (color maps) allow to study the distribution of dust in the star-forming regions, its spatial correlation with star formation processes, and to constrain its mass (e.g., Brosch \& Loinger 1991).

\section{A.5 Environmental influences on the star-forming activity and evolution of dwarf galaxies}

The long-term spectrophotometric evolution of star-forming dwarf galaxies does not seem to be regulated by their intrinsic properties, such as radial mass distribution of the luminous and dark matter, alone. Also environmental influences are likely to be important, as suggested by the fact that quiescent dEs are predominantly found in high density environments, while BCDs generally populate regions of low density of luminous galaxies, concentrated towards voids or void boundaries (Salzer 1989, Lindner et al. L96, see Paper VI of this thesis for details). To what degree the various environmental factors are capable of influencing, e.g., a dwarf galaxy's star-forming activity remains a subject of debate (cf. e.g. Vílchez 1995 and Pustilnik et al. 2001 for reviews, or see Paper VI of this thesis).

Interaction processes due to movement within dense $\left(\sim 10^{-3} \mathrm{~cm}^{-3}\right)$ intracluster gas, such as ram-pressure stripping of the gas component or shock-induced star formation (Vílchez 1995), can be largely ruled out in low-density environments. Similarly, massive companions which might act as starburst triggers are rare in the environment of BCDs. If present, such luminous companion galaxies mostly reside at large projected distances to BCDs, and show on average no significant influence on their star-forming activity (Campos-Aguilar \& Moles 1991, Telles \& Terlevich TT95).

Other studies have raised the question whether low-mass companions, optically faint or purely gaseous objects, in the close environment of star-forming dwarf galaxies may be frequent triggering agents of their starburst activity. Low-mass companions at short distances can not only exert strong tidal forces, but may also influence starburst activity by donating gas or merging with the $\mathrm{BCD}$, as suggested by observations of some luminous BCDs (Östlin et al. 2001). Surveys of faint stellar or gaseous companions in the close environment of BCDs and dIs suggest that such companions may be frequent, but mostly undetectable at large distances(Taylor et al. 1995, 1996, Lindner et al. L96, Pustilnik et al. 2001, or see Papers V and VI). The influence of such compan- 
ions on the star-forming activity of BCDs has, however, not yet been systematically studied (see Paper VI).

\section{A.6 Structure of this thesis}

This thesis deals with several of the aforementioned questions concerning Blue Compact Dwarf Galaxies. The different research projects that were carried out in the context of this work are presented in six papers, which form the chapters I-VI of this thesis.

Papers I-III describe a first systematic Near-Infrared study of the photometric structure of BCDs, addressing in particular the structure of the stellar LSB host galaxy, and its relation to star-forming activity in BCDs. A systematic investigation of the morphological subclass of cometary BCDs, and the relation of such objects to the class of typical elliptical BCDs, is presented in Paper IV. In Papers V and VI, an environmental study of star-forming dwarf galaxies is summarized, concentrating on the frequency of faint companion galaxies and their influence on star-forming activity in gas-rich dwarf galaxies.

The following chapter B gives a summary of the studies described in Papers I-VI. Chapter $\mathrm{C}$ highlights the impact of specific results of this thesis on currently debated questions in the field of dwarf galaxy research. This chapter also gives an outlook on future research projects which may be of particular importance to the topics investigated within this work. 


\section{References}

Paper I: Noeske, K.G., Papaderos, P., Cairós, L.M. \& Fricke, K.J. 2003a, A\&A, submitted Paper II: Noeske, K.G., Papaderos, P., Cairós, L.M. \& Fricke, K.J. 2003b, A\&A, in preparation

Paper III: Noeske, K.G., Papaderos, P., Cairós, L.M. \& Fricke, K.J. 2003c, A\&A, in preparation

Paper IV: Noeske, K. G., Guseva, N. G., Fricke, K. J., Izotov, Y. I., Papaderos, P. \& Thuan, T. X. 2000, A\&A, 361, 31

Paper V: Noeske, K. G., Iglesias-Páramo, J., Vílchez, J. M. \& Fricke, K. J. 2001b, Ap\&SS, 276, 577

Paper VI: Noeske, K. G., Iglesias-Páramo, J., Vílchez, J. M., Papaderos, P. \& Fricke, K. J. 2001a, A\&A, 371, 806

Bergvall, N. \& Östlin, G. 2002, A\&A, 390, 891

Binggeli, B. \& Cameron, L. M. 1991, A\&A, 252, 27

Brosch, N. \& Loinger, F. 1991, A\&A, 249, 327

Cairós, L. M., Caon, N., Papaderos, P., Noeske, K. G., Vílchez, J. M., García Lorenzo, B., Muñoz-Tuñon, C. 2002a, submitted

Cairós, L. M., Caon, N., Vílchez, J. M., González-Pérez, J. \& Muñoz-Tuñón, C. 2001b, ApJS, 136, 393

Cairós, L. M., Vílchez, J. M., González Pérez, J., Iglesias-Páramo, J. \& Caon, N. 2001a, ApJS, 133, 321

Campos-Aguilar, A., Moles, M. 1991, A\&A, 241, 358

Chiu, W. A., Gnedin, N. Y., \& Ostriker, J. P. 2001, ApJ, 563, 21

Dalcanton, J. J. \& Hogan, C. J. 2001, ApJ, 561, 35

Davies, J. I. \& Phillipps, S. 1988, MNRAS, 233, 553

De Young, D. S. \& Heckman, T. M. 1994, ApJ, 431, 598

Elmegreen, B. G., Elmegreen, D. M., Salzer, J. S. \& Mann, H. 1996, ApJ, 467, 579

Fricke, K. J., Izotov, Y. I., Papaderos, P., Guseva, N. G. \& Thuan, T. X. 2001, AJ, 121, 169

Gallagher, J. S. 1998, The Magellanic Clouds and Other Dwarf Galaxies, Eds. T. Richtler \& J. M. Braun, Shaker Verlag, Aachen, p. 25

Gerola, H., Seiden, P. \& Schulmann, L. 1980, ApJ, 242, 517

Gil de Paz, A., Silich, S. A., Madore, B. F., Sánchez Contreras, C., Zamorano, J. \& Gallego, J. 2002, ApJ, 573, L101

Gil de Paz, A., Zamorano, J. \& Gallego, J. 2000b, A\&A, 361, 465

Hunt, L. K., Thuan, T. X., \& Izotov, Y. I. 2003, ApJ, in press

Hunter, D. 1997, PASP, 109, 937

Hunter, D.A., Elemegreen, B.G. \& Baker, A.L. 1998, ApJ 493, 595

Izotov, Y.I., Thuan, T.X., 1999, ApJ 511, 639

Izotov, Y. I., Thuan, T. X. \& Lipovetsky, V. A. 1997a, ApJS, 108, 1

Izotov, Y. I., Lipovetsky, V. A., Chaffee, F. H., Foltz, C. B., Guseva, N. G. \& Kniazev, A. Y. 1997b, ApJ, 476, 69

Krüger, H., Fritze-v. Alvensleben, U. \& Loose, H.-H. 1995, A\&A, 303, 41

Kunth, D. \& Sargent, W. L. W. 1986, ApJ, 300, 496

Lindner, U., Einasto, M., Einasto, J., Freudling, W., Fricke, K., Lipovetsky, V., Pustilnik, S., Izotov, Y., Richter, G. 1996, A\&A, 314, 1

Lisenfeld, U., Israel, F. P., Stil, J. M. \& Sievers, A. 2002, A\&A, 382, 860

Loose, H. H. \& Thuan, T. X. 1986, Star Forming Dwarf Galaxies and Related Objects, 73 
Mac Low, M. \& Ferrara, A. 1999, ApJ, 513, 142

Marlowe, A. T., Meurer, G. R. \& Heckman, T. M. 1999, ApJ, 522, 183

Meurer, G. R., Staveley-Smith, L. \& Killeen, N. E. B. 1998, MNRAS, 300, 705

Östlin, G. 2000, ApJ, 535, L99

Östlin, G., Amram, P., Bergvall, N., Masegosa, J., Boulesteix, J. \& Márquez, I. 2001, A\&A, 374,800

Östlin, G. \& Kunth, D. 2001, A\&A, 371, 429

Pagel, B. E. J., Simonson, E. A., Terlevich, R. J. \& Edmunds, M. G. 1992, MNRAS, 255, 325

Papaderos, P. 1998, PhD Thesis, University of Göttingen

Papaderos, P., Fricke, K. J., Thuan, T. X., Izotov, Y. I. \& Nicklas, H. 1999, A\&A, 352, L57 (P99)

Papaderos, P., Izotov, Y.I., Thuan, T.X., Noeske, K.G., Guseva, N.G. \& Fricke, K.J. 2002, A\&A, 393, 461

Papaderos, P., Loose, H.-H., Thuan, T.X. \& Fricke, K.J. 1996a, A\&AS, 120, 207

Papaderos, P., Loose, H.-H., Fricke, K. J., Thuan, T. X. 1996b, A\&A,314, 59

Peimbert, M. \& Torres-Peimbert, S. 1974, ApJ, 193, 327

Pustilnik, S.A., Kniazev, A.Y., Ugryumov, A.V. 2001, A\&A, 373, 24

Salzer, J.J. 1989, ApJ, 347, 152

Salzer, J.J., McAlpine, G.M. \& Boroson, T.A. 1989a, ApJS, 70, 447

Salzer, J.J., McAlpine, G.M., Boroson, T.A. 1989b, ApJS, 70, 479

Schulte-Ladbeck, R. E., Hopp, U., Greggio, L., Crone, M. M. \& Drozdovsky, I. O. 2001, AJ, 121, 3007

Silich, S. \& Tenorio-Tagle, G. 2001, ApJ, 552, 91

Taylor, C.L., Brinks, E., Grashius, R.M., Skillman, E.D. 1995, ApJS, 102, 189

Taylor, C.L., Thomas, D.L., Brinks, E., Skillman, E.D. 1996, ApJS, 107, 143

Telles, E. \& Terlevich, R. 1995, MNRAS, 275, 1 (TT95)

Telles, E. \& Terlevich, R. 1997b, MNRAS, 286, 183

Telles, E., Melnick, J. \& Terlevich, R. 1997, MNRAS, 288, 78

Terlevich, R., Melnick, J., Masegosa, J., Moles, M. \& Copetti, M.V.F. 1991, A\&AS, 91, 885

Thuan, T.X. 1991, Massive Stars in Starbursts, Eds. C. Leitherer, N.R., Walborn, T. M. Heckman, C. A. Norman, Cambridge University Press, p. 183

Thuan, T.X. \& Martin, G. 1981, ApJ, 247, 823

van den Bergh, S. 2000, PASP, 112, 529

van den Bosch, F. C. \& Swaters, R. A. 2001, MNRAS, 325, 1017

van Zee, L., Salzer, J. J. \& Skillman, E.D. 2001, AJ, 122, 121

Vennik, J., Hopp, U., \& Popescu, C. C. 2000, A\&AS, 142, 399

Vílchez, J.M. 1995, AJ, 110, 1090

Zwicky, F. 1957, Morphological Astronomy, Springer Verlag, Berlin, Heidelberg, New York 


\section{Summary of the individual research projects and results}

\section{B.1 Near Infrared studies of the stellar host galaxy and starburst activity in BCDs}

The central topic of this thesis is a first deep Near Infrared (NIR) imaging study of a larger sample of BCDs, presented in Papers I - III. Aiming at an improved separation of old and young stellar populations with respect to previous studies in visible wavelengths, deep images in the $J, H$ and $K$ broad band filters were observed for a sample of $30 \mathrm{BCDs}$ and 3 similar objects (Papers I and II).

For each of the sample galaxies, morphological information and photometry as derived from the latter NIR images are presented, in particular for the star-forming regions. These data are briefly discussed in view of information which was previously published for the respective galaxy.

The limiting surface brightness of the data set allows throughout to unambiguously detect and analyze the radial intensity distribution of the old stellar low-surface brightness (LSB) host galaxy of these objects. The latter information is required to decompose the derived surface brightness profiles (SBPs) into the light contributions of the young and old stellar populations (Paper I, Sect. 3.2). Above all, the radial intensity distribution of the stellar LSB host galaxy holds relevant information for the understanding of the starburst activity, dynamics and evolution of BCDs.

The present work provides new information on the LSB host, in particular close to its center, owing to the lower line-of-sight intensity contribution of the central starburst in the NIR than in optical wavelengths. These results are analyzed with respect to the global structure of the LSB host and its relation to the star-forming activity of gas-rich dwarf galaxies, as detailed in Paper III. A summary of this analysis is given in the following sections.

\section{B.1.1 New insights into the structure of the stellar host galaxy}

NIR SBPs of the LSB host galaxies agree at large galactocentric radii with those from optical studies in the $B$ band, showing also an exponential intensity decrease, with compatible scale lengths $\left(\alpha_{E}\right)$ and extrapolated central surface brightnesses $\left(\mu_{E, 0}\right)$. Some objects display in $B$ a less concentrated host galaxy structure, with smaller $\alpha_{E}$ and fainter $\mu_{E, 0}$. These differences are mostly attributable to residuals from extranuclear star-forming activity in the $B$ band, and to possibly non-exponential surface 
brightness profiles of the LSB host $\left(\mathrm{SBP}_{\mathrm{LSB}}\right)$, fitted over differing radii in $B$ and in the NIR (Paper III, Sect. 3.1 \& Fig. 2). The relations between the structural parameters $\mu_{E, 0}$ and $\alpha_{E}$ of the stellar host and its luminosity $\left(M_{\mathrm{E}}\right)$, found in the $B$ band, are reproduced by the NIR data (Paper III, Sect. 3.2). These results confirm the systematically more compact structure of the stellar hosts of BCDs, compared to dwarf irregular (dI) and dwarf elliptical (dE) galaxies, which had previously been found in the $B$ band.

Frequently, the NIR data reveal evidence for a flattening of the exponential intensity profile of the LSB component at small to intermediate radii, i.e. within few exponential scale lengths $\alpha_{E}$ of the stellar host (Paper I, Sect. $3.2 \&$ Fig. 15). Such profiles (type V SBPs, Binggeli \& Cameron 1991) have rarely been detected in the LSB component of BCDs at optical wavelengths, where the relative flux contribution of the starburst, being stronger than in the NIR, can readily hide a possible central intensity depression in the underlying LSB host. Type V SBP $\mathrm{LSB}_{\mathrm{LB}}$ are found for $55 \pm 16 \%$ of the sample galaxies. This first estimate of the frequency of type V SBPs in BCDs should be considered an indicative lower limit only. The data suggest that a considerable fraction of type $\mathrm{V}$ $\mathrm{SBP}_{\mathrm{LSB}}$ with a relatively small central flattening may remain undetected also in the present NIR study, being hidden by the starburst emission (Paper III, Sect. 4.1.1).

The physical origin of such type V LSB profiles in BCDs and dwarf galaxies in general has not yet been studied. The adoption of an empirical fitting function, a modified exponential (Papaderos et al. 1996a), allows however to approximate and quantitatively study such profiles, and to decompose the total SBPs in a physically meaningful way (Paper I, Sect. 3.2).

For most type $\mathrm{V} \mathrm{SBP}_{\mathrm{LSB}}$, the relative depression $q$ with respect to the central intensity extrapolated from the outer exponential regime $\left(\mu_{E, 0}\right)$ shows a tight relation to their cutoff radius $b$ (if the latter is normalized to the outer exponential scale length $\alpha_{E}$ ) within which flattening becomes significant. Neither $b$ nor $q$ show systematic dependences on the structure or luminosity of the LSB host. However, $b$ and $q$ are found to be non-linearly related to each other (Paper III, Sect. 4.1.2).

For those galaxies with a type $\mathrm{V} \mathrm{SBP} \mathrm{LSB}_{\text {in }}$ which was undetected in the $B$ band, a decomposition by means of the commonly used exponential fit to the host galaxy may result in an overestimation of the host galaxy flux by a factor of the order of 1.5 . The starburst light fraction, and hence the predicted amount of fading after the end of starburst activity, is correspondingly underestimated in such cases (Paper III, Sect. 5.1).

\section{B.1.2 Relations between the stellar LSB host and star-forming activity}

In Paper III, an attempt is presented to enlighten the empirical relations between the properties of the starburst activity of a dwarf galaxy, and those of its stellar host galaxy. For this purpose, a subsample of typical BCDs, probably not significantly influenced by interactions with their environment, is selected. For these objects, constraints to their radial stellar mass distributions are derived, following clues from previous empirical studies which have pointed to a probably important influence of this quantity.

Based on these stellar mass distributions, the existence of a critical stellar luminosity density of the LSB host $\left(l_{\text {crit }}\right)$ of the order $0.2 L_{\odot, J} \mathrm{pc}^{-3}$ (corresponding to a critical 
stellar mass density $\rho_{\text {crit }} \sim 0.4 M_{\odot} \mathrm{pc}^{-3}$ ) is hypothesized, above which burst-like SF activity becomes possible, but is not forced (Paper III, Sect. 5.2.1).

The empirical scale length - luminosity $\left(\alpha_{E}-M_{\mathrm{E}}\right)$ and central surface brightness luminosity $\left(\mu_{E, 0}-M_{\mathrm{E}}\right)$ relations of the stellar host (Papaderos et al. 1996b, Marlowe et al. 1999, Salzer \& Norton 1999) of BCDs strongly support this hypothesis. The upper envelopes and linear fits to these relations follow lines of constant central density of the stellar host galaxy. The hypothesis of the existence of $l_{\text {crit }}$ for burst-like star formation allows therefore to analytically predict the empirical $\left(\alpha_{E}-M_{\mathrm{E}}\right)$ and $\left(\mu_{E, 0}-\right.$ $M_{\mathrm{E}}$ ) relations of the stellar hosts of BCDs, on the assumption of stellar host galaxies with an exponential surface brightness distribution and a roughly spheroidal geometry (Paper III, Sect. 5.2.2 \& Fig. 6).

The hypothesis of a threshold density can also account for the relations between the extent of star-forming activity and the luminosity $M_{\mathrm{E}}$ of the stellar hosts (Papaderos et al. 1996b). For a stellar host with given structural parameters, the galactocentric radius where the luminosity density equals $l_{\text {crit }}$ can be derived, i.e. the radius within which the star-forming activity should be confined. Such calculations are found to be capable of predicting the observed relation between the starburst radius and $M_{\mathrm{E}}$, and the relation between the fractional extent of the starburst within the LSB host and $M_{\mathrm{E}}$ (Paper III, Sect. 5.2.4 \& Fig. 7).

The spread of the latter relations, and of the $\left(\alpha_{E}-M_{\mathrm{E}}\right)$ and $\left(\mu_{E, 0}-M_{\mathrm{E}}\right)$ relations, can be explained by the intrinsic range of structural parameters of stellar hosts of BCDs. The $\left(\alpha_{E}-M_{\mathrm{E}}\right)$ and $\left(\mu_{E, 0}-M_{\mathrm{E}}\right)$ relations suggest the existence of an upper limit of the central stellar mass density of dwarf galaxies. This might reflect an intrinsic property of the dynamics of such systems. Alternatively, increasingly intense starburst activity towards higher central mass densities of the stellar hosts might regulate the central matter density of BCDs (Paper III, Sect. 5.2.3).

A comparison of dIs and BCDs shows that their separation in the $\left(\alpha_{E}-M_{\mathrm{E}}\right)$ and $\left(\mu_{E, 0}-M_{\mathrm{E}}\right)$ parameter spaces occurs along a certain interval of central densities of their stellar hosts, close to $l_{\text {crit }}$ (Paper III, Sect. 5.2.3). The systematically more compact LSB host structure of BCDs with respect to dIs (Papaderos et al. 1996b, Marlowe et al. 1999, Salzer \& Norton 1999) may therefore be understood as a manifestation of this density threshold $l_{\text {crit }}$ above which burst-like star formation can occur. Gasrich dwarf galaxies with a sufficiently dense stellar host galaxy are apparently capable of sustaining burst-like star formation, by which they are classified as BCDs. Those objects which fall short of fulfilling this criterion do largely experience a low-level mode of star-formation, and are therefore classified as dIs. In particular, literature data show that those star-forming dwarfs with the highest mass densities of their stellar hosts are almost throughout classified as BCDs. The sparsity of gas-rich quiescent objects with a comparably compact LSB structure suggests that BCDs obtain a less compact host galaxy structure after the termination of a starburst episode. This result supports a dynamic evolutionary scenario, in which a BCD can undergo a redistribution of its gaseous mass, due to the energy release of strong star-forming activity. This will then initiate an adiabatic expansion of the stellar component. A static, Dark Matterdominated evolutionary scenario is only tenable on the assumption that compact BCDs 
undergo strong star formation activity over several $10^{9} \mathrm{yr}$, with at most short less active phases (Paper III, Sect. 5.2.3).

The luminosity ratios of the starburst and the stellar host, $L_{S B} / L_{\mathrm{E}}$, show a large spread, with mean values of $\sim 0.6$ and $\sim 1.8$ in the $J$ and $B$ bands, respectively, but display no significant correlation with the luminosity of the stellar host (Paper III, Sect. 5.1). A follow-up study of this result suggests that in a major fraction of BCDs the luminosity density of the starburst, averaged over the starburst volume, is approximately proportional to the central mass density of the stellar host galaxy (Paper III, Sect. 5.2.5).

The results presented in Paper III strongly suggest that the stellar mass density of the host galaxy is a key parameter of physical processes which majorly influence starforming activity. In this respect, a modified Jeans-criterion in the presence of a compact stellar background (Loose \& Fricke 1980, Loose et al. 1982) is proposed as an appealing scenario (Paper III, Sect. 5.2.6). This mechanism relates the maximum density of individual star-forming clouds, prior to their collapse and the onset of star formation, to the average density of the underlying stellar background. On simple assumptions, this scenario appears capable of accounting for the empirical dependences of starburst activity on the mass density of the stellar host, suggested by the present investigation.

\section{B.2 The relation of the "cometary" morphological class of BCDs to typical elliptical BCDs}

An extensive study of two prototypical cometary ("iI,C") BCDs, Markarian 59 and Markarian 71, is presented in Paper IV. Imaging and spectroscopic data from groundbased observations, and archival Hubble Space Telescope (HST) images, are analyzed by a variety of methods (Paper IV, Sect. 2). The results are jointly discussed with respect to the morphology of iI,C BCDs, as well as their relation to typical elliptical BCDs.

The radial intensity distributions of the underlying stellar LSB host galaxy of both iI,C BCDs can be approximated by an exponential fitting law. The derived central surface brightnesses and exponential scale lengths point to a moderately compact structure of the stellar hosts, intermediate between those of typical iE/nE BCDs and more diffuse dIs and dEs (Paper IV, Sect. 3.1 \& Fig. 9).

From long-slit spectra, average oxygen abundances of $12+\log (\mathrm{O} / \mathrm{H})=8.0$ and $12+\log (\mathrm{O} / \mathrm{H})=7.8$ are inferred for the warm interstellar medium of Markarian 59 and Markarian 71, respectively. The abundances of various elements show only small spatial variations, both in the vicinity of the starburst regions, and along the elongated host galaxy, suggesting an efficient mixing of heavy elements (Paper IV, Sect. 3.2).

To put constraints on the ages of the stellar host galaxies, long-slit spectra are corrected for nebular emission and subsequently analyzed by means of population synthesis models. In combination with observed emission line equivalent widths, and $B-V$ color magnitude diagrams from HST images, the solutions yield a most probable age of $\lesssim 2$ Gyr for the host galaxy of either BCD. Even when model uncertainties are taken 
into account, ages higher than 4 Gyr for Markarian 59 and 3 Gyr for Markarian 71 are implausible (Paper IV, Sect. 3.4).

The resemblance of both latter cometary BCDs, regarding the ages and structural properties of their stellar host galaxies, is followed up through a comparison to other objects of the same morphological class (Paper IV, Sect. 4.2). Those cometary BCDs for which suitable comparison data were previously published show similar properties. The peculiar starburst morphology of such objects may therefore be related to specific physical properties of the host galaxies, rather than be fortuitous. Hypotheses which appear worth being followed up are:

(i) Cometary BCDs are relatively young objects, with ages not exceeding a few Gyr, thus systematically younger than the typical BCDs of type $\mathrm{iE} / \mathrm{nE}$. If true, the development of iI,C morphology may represent an advanced evolutionary stage of a young $\mathrm{BCD}$, before it gradually attains characteristics of old, evolved $\mathrm{iE} / \mathrm{nE}-\mathrm{BCDs}$.

(ii) The stellar host galaxies of iI,C BCDs, except for those of metal-deficient young galaxy candidates, are moderately compact. With respect to their surface brightnesses and exponential scale lengths, they may form intermediate cases between typical compact $\mathrm{iE} / \mathrm{nE}$ BCDs and more diffuse dIs/dEs.

Both the location of starburst activity, and signatures of propagating star-forming activity along the elongated stellar bodies of iI,C BCDs, suggest that the surface density distribution of cold gas in these systems resembles their optical morphology. Published H I maps support this assumption (Paper IV, Sect. 4.3).

\section{B.3 Environmental studies of star-forming dwarf galaxies}

Papers V and VI concentrate on the environment of star-forming dwarf galaxies. Results from recent studies have pointed to the presence of numerous companions of low intrinsic luminosity in the close environment of star-forming dwarfs, and have raised the question whether such faint companions can significantly influence the starforming activity of a dwarf galaxy.

As detailed in Paper V, a sample of 142 BCDs and other star-forming dwarf galaxies is selected. For a sample of 90 distant objects with heliocentric recession velocities $c z>2000 \mathrm{~km} \mathrm{~s}^{-1}$, the large-scale environment is searched for companions, using the NASA Extragalactic Data Base. The results show that only $50 \%$ of the sample galaxies can be considered isolated, in the sense that no companions are found within a projected linear separation $s_{p}<1 \mathrm{Mpc}$, and a projected recession velocity difference of $\Delta c z<500 \mathrm{~km} \mathrm{~s}^{-1}$ (Paper V, Sect. 4). Those probably more strongly isolated objects are compared to the total distant sample with respect to their star-forming activity. The distributions of the $\mathrm{H} \beta$ emission line equivalent widths and integral $(B-V)$ colors of either sample, respectively reflecting current and recent star-forming activity, show no significant difference. Companion search results are compared for subsamples in different recession velocity intervals, and show a strong bias against dwarf companions at large distances (Paper I, Sect. $5.1 \& 5.2$ ). 
These results are followed up in Paper VI. Concentrating on the close environment of star-forming dwarfs, a maximum projected linear separation $s_{p}<100 \mathrm{kpc}$, and a maximum recession velocity difference to the sample galaxies of $\Delta c z<500 \mathrm{~km} \mathrm{~s}^{-1}$ are adopted to select likely close companions. Among the found close companions, $>80 \%$ are classified as dwarf galaxies $\left(\mathbf{M}_{B}>-18\right)$. Beyond distances corresponding to a recession velocity $c z>4000 \mathrm{~km} \mathrm{~s}^{-1}$ ( $D \gtrsim 50 \mathrm{Mpc}$ ), such objects are found to be absent in the NASA Extragalactic Data Base, reflecting the observational bias against faint galaxies at large distances. The investigation of a nearby subsample ( $c z<$ $2000 \mathrm{~km} \mathrm{~s}^{-1}$ ), to moderate this observational bias, reveals close dwarf companions for approximately $30 \%$ of the star-forming dwarf galaxies under study. This fraction must be considered a lower limit to the true value, given the limited completeness of the available data set for faint companions (Paper VI, Sect. 3.1.2 \& 4).

The recession velocity differences between the star-forming dwarfs and their identified companions amount typically to $\lesssim 250 \mathrm{~km} \mathrm{~s}^{-1}$. Their frequency rises towards lower velocity separations. This trend has similarly been reported for dwarf companions of luminous spiral galaxies, and suggests a physical association between the star-forming dwarfs and their companions (Paper VI, Sect. 4 \& Fig. 1).

The $B-V$ colours and the $\mathrm{H} \beta$ equivalent widths show no significant differences, if compared for objects with and without a possible close companion. However, the available statistics do not allow to conclude whether star-forming activity is regulated by internal processes only. It is pointed out that comparisons of the above mentioned indicators of star-forming activity suffer from considerable intrinsic scatter which, along with the insufficient completeness of companion search catalogs, may render small systematic differences undetectable (Paper VI, Sect. $3.2 \& 4$ ). 


\section{References}

Paper I: Noeske, K.G., Papaderos, P., Cairós, L.M. \& Fricke, K.J. 2003a, A\&A, submitted Paper II: Noeske, K.G., Papaderos, P., Cairós, L.M. \& Fricke, K.J. 2003b, A\&A, in preparation

Paper III: Noeske, K.G., Papaderos, P., Cairós, L.M. \& Fricke, K.J. 2003c, A\&A, in preparation

Paper IV: Noeske, K. G., Guseva, N. G., Fricke, K. J., Izotov, Y. I., Papaderos, P. \& Thuan, T. X. 2000, A\&A, 361, 31

Paper V: Noeske, K. G., Iglesias-Páramo, J., Vílchez, J. M. \& Fricke, K. J. 2001b, Ap\&SS, 276, 577

Paper VI: Noeske, K. G., Iglesias-Páramo, J., Vílchez, J. M., Papaderos, P. \& Fricke, K. J. 2001a, A\&A, 371, 806

Binggeli, B. \& Cameron, L. M. 1991, A\&A, 252, 27

Loose, H. \& Fricke, K. 1980, Astrophys. Letters, 21, 65

Loose, H. H., Kruegel, E. \& Tutukov, A. 1982, A\&A, 105, 342

Marlowe, A. T., Meurer, G. R. \& Heckman, T. M. 1999, ApJ, 522, 183

Papaderos, P., Loose, H.-H., Thuan, T.X. \& Fricke, K.J. 1996a, A\&AS, 120, 207

Papaderos, P., Loose, H.-H., Fricke, K. J., Thuan, T. X. 1996b, A\&A,314, 59

Salzer, J. J. \& Norton, S. A. 1999, ASP Conf. Ser. 170: The Low Surface Brightness Universe, 253 



\section{Scientific impact of this work and outlook on future research}

\section{C.1 The NIR study}

The first systematic structural study of BCDs in the Near Infrared has revealed new details of their photometric structure, which had remained largely undiscovered in optical wavelengths.

Of particular relevance is the previously unexpected discovery of inwards-flattening exponential (type V) surface brightness profiles in more than $50 \%$ of BCDs. The occurrence of such profiles in an appreciable fraction of BCDs imposes important new observational constraints to the radial mass distribution of the stellar LSB component. This information is of prime importance to studies of the star-forming activity and evolutionary processes in dwarf galaxies. Also a correct estimation of the photometric fading of these systems after the termination of star-forming activity depends critically on the detection of type $\mathrm{V}$ profiles. This information will be relevant to studies of the future evolution of BCDs, and of their relation to quiescent dwarf elliptical galaxies (Paper I, Sect. 5.2; Paper III, Sect. 4.1 \& 5.1).

The pilot study of type $\mathrm{V}$ profiles, carried out in this thesis, emphasizes the importance of future deep NIR studies of local star-forming dwarf and compact galaxies. It may be equally relevant to study similar objects at higher redshifts, "Faint Blue Galaxies" or "Compact Narrow Emission Line Galaxies", at rest-frame NIR wavelengths. Studies of possible type V profiles may aid to constrain the future photometric fading of such systems, and to understand their currently debated relations to quiescent galaxies in the local Universe. The apparent faintness and small angular size of these compact starburst galaxies at high redshift poses a challenge to observations, to be met by large ground-based telescopes in combination with adaptive optics, and space observatories.

\section{C.2 The relations between the stellar host galaxy and starburst activity}

The results inferred from the NIR data have allowed to gain new clues to the origin and regulating mechanisms of starburst activity in BCDs, issues which are to date not understood and actively debated (Paper III, Sect. 5). Investigations presented within this thesis provide strong support for the previous hypothesis that the occurrence and 
properties of starburst activity are closely related to the stellar mass density of the host galaxy. Empirically found relations between the properties of the starburst and those of the host galaxy may for the first time be consistently explained, on the hypothesis that burst-like star-forming activity is restricted to regions where the stellar mass density of the LSB host galaxy exceeds a certain threshold value.

The identification of the stellar mass density as a regulating agent of burst-like star formation in gas-rich dwarfs holds the promise of a better understanding of such systems. As was discussed in the present work, the interplay between the mass distribution and star-forming activity in dwarf galaxies is likely a key process of their evolution, governing their manifold physical properties and their evolutionary relations among each other.

Future detailed studies, using spectroscopic data of individual star-forming regions, and NIR data to constrain the mass density of the stellar host galaxy, will allow to further elucidate the physical mechanisms of starburst activity in dwarf galaxies. Above all, such data will provide important quantitative constraints to the theory of star formation in such objects, and in particular to models of their starburst-driven evolution.

\section{C.3 The question of Dark Matter in BCDs}

The present work lends circumstantial support to the view that BCDs may not be entirely dominated by Dark Matter within their Holmberg radius. Instead, the gaseous and stellar mass may dominate the dynamics of such systems within their optical extent.

In particular, the dependence of the starburst activity on the mass density of the stellar host galaxy strongly suggests that stellar matter dominates the potential within which gas collapses to form stars (Paper III, Sect. 5.2.7). Similarly, a non Dark Matterdominated, dynamic scenario of dwarf galaxy evolution can most plausibly account for the lack of quiescent dwarf galaxies with very compact stellar host galaxies (Paper III, Sect. 5.2.3).

In agreement with recently published results, these lines of evidence emphasize the importance of studies of the stellar host galaxies of BCDs, to improve the understanding of the dynamics and evolution of these systems. More detailed information on the dynamics of BCDs may be obtained from detailed kinematical studies of their stellar hosts, by means of high-resolution spectroscopy of stellar absorption lines. Since such a project can only be performed outside the influence of star-forming regions, at surface brightness levels $\mu \gtrsim 24.5 \mathrm{Bmag} / \mathrm{arcsec}^{2}$, and will therefore be very demanding in terms of observing time even at the largest currently available telescopes.

\section{C.4 Cometary BCDs}

The finding that cometary BCDs may systematically differ from typical elliptical BCDs, regarding the ages and structure of their host galaxies, awaits further confirmation (Paper IV, Sect. $4.2 \&$ 4.3.1). This result suggests, however, that a comparison 
of different morphological subclasses of BCDs may be rewarding, holding clues to their possible evolutionary relations and differing mechanisms of star-forming activity. Such comparisons, using homogeneous data sets for larger samples of objects, are currently planned. Among other data, the NIR images analyzed in this thesis will be employed for this purpose.

In addition, interferometric studies of the gas component of cometary BCDs may allow to assess the intrinsic processes regulating SF in such intriguing, yet relatively frequent objects.

\section{C.5 Faint companions in the close environment of star-forming dwarf galaxies}

The identification of companions in the close environment of star-forming dwarf galaxies has readdressed the question whether gravitational interactions can be partly responsible for the onset of their star-forming activity. During previous studies, interaction-induced star-forming activity had been dismissed for most of these objects, due to the absence of luminous companions (Paper VI, Sect. 4 \& 5). Future deep spectrophotometric surveys, such as SLOAN, will be helpful to closely constrain the actual frequency of faint companions, and their influence on star-forming dwarf galaxies. However, for a deeper understanding of the latter issue, a theoretical treatment of dwarf galaxy interactions may be indispensable.

The evidence suggesting that star-forming dwarf galaxies may frequently be physically associated with other dwarf galaxies is particularly intriguing. Such a result, if further confirmed and quantified, may hold new information on the formation mechanisms of dwarf galaxies, and may also be interesting to theoretical studies of cosmological structure formation. 



\title{
New insights to the photometric structure of Blue Compact Dwarf Galaxies from deep Near-Infrared studies $^{0}$
}

\section{Observations, surface photometry and decomposition of surface brightness profiles}

\author{
K.G. Noeske, P. Papaderos, L.M. Cairós, K.J. Fricke \\ Universitäts-Sternwarte, Geismarlandstraße 11, D-37083 Göttingen, Germany
}

Summary. We have analyzed deep Near Infrared (NIR) broad band images for a sample of Blue Compact Dwarf Galaxies (BCDs), observed with the ESO NTT ${ }^{1}$ and Calar Alto ${ }^{2} 3.6 \mathrm{~m}$ telescopes. The data presented here allows for the detection and quantitative study of the extended stellar low-surface brightness (LSB) host galaxy in all sample BCDs. NIR surface brightness profiles (SBPs) of the LSB host galaxies agree at large galactocentric radii with those from optical studies, showing also an exponential intensity decrease and compatible scale lengths. At small to intermediate radii (within 1-3 exponential scale lengths), however, the NIR data reveals for more than one half of our sample BCDs evidence for a significant flattening of the exponential profile of the LSB component. Such profiles (type V SBPs, Binggeli \& Cameron 1991) have rarely been detected in the LSB component of BCDs at optical wavelengths, where the relative flux contribution of the starburst, being stronger than in the NIR, can readily hide a possible central intensity depression in the underlying LSB host. The structural properties, frequency and physical origin of type V LSB profiles in BCDs and dwarf galaxies in general have not yet been subject to systematic studies. Nevertheless, the occurrence of such profiles in an appreciable fraction of BCDs would impose important new observational constraints to the radial mass distribution of the stellar LSB component, as well as to the photometric fading of these systems after the termination of star-forming activities. We test the suitability of two empirical fitting functions, a modified exponential distribution (Papaderos et al. 1996a) and the Sérsic law, for the systematization of the structural properties of BCD host galaxies which show a type $\mathrm{V}$ intensity distribution. Either function has been found to satisfactorily fit a type $\mathrm{V}$ distribution. However, it is argued that the practical applicability of Sérsic fits to the LSB emission of BCDs is limited by the extreme sensitivity of the achieved solutions to, e.g., small uncertainties in the sky subtraction and SBP derivation. We find that most of the sample BCDs show in their stellar LSB host galaxy optical-NIR colors indicative of an evolved stellar population with subsolar metallicity. Unsharp-masked NIR maps reveal numerous morphological details and indicate in some cases, in combination with optical data, appreciable non-uniform dust absorption on a spatial scale as large as $\sim 1 \mathrm{kpc}$.

\footnotetext{
${ }^{0}$ This paper has been submitted in this form to Astronomy \& Astrophysics.

${ }^{1}$ European Southern Observatory, program ID 65.N-0318(A)

${ }^{2}$ German-Spanish Astronomical Center, Calar Alto, operated by the Max-Planck-Institute for Astronomy, Heidelberg, jointly with the Spanish National Commission for Astronomy.
} 


\section{I.1 Introduction}

The star-formation history and chemodynamic evolution of Blue Compact Dwarf (BCD) galaxies are central issues in the contemporary dwarf galaxy research. In spite of being old in their vast majority, BCDs resemble in many aspects unevolved lowmass galaxies in the early Universe. They are gas-rich (H I mass fraction of typically $>30 \%)$ and metal-deficient $\left(Z_{\odot} / 50 \lesssim Z \lesssim Z \odot / 3\right)$ extragalactic systems, undergoing intense, spatially extended star-forming (SF) activity. Such properties are believed to have been common among young low-mass objects at high to intermediate redshift, such as pre-galactic building blocks (Lowenthal et al. 1997, Hirashita et al. 2000, Fujita et al. 2001) or the progenitors of the present-day dwarf spheroidals (e.g. Babul \& Rees 1992; Guzmán et al. 1998). BCDs are therefore convenient nearby laboratories to study at high spatial resolution the impact of collective star formation on the spectrophotometric and chemodynamic properties of these distant and faint extragalactic sources. Moreover, they are important testbeds for deducing constraints to cosmological parameters, such as the primordial ${ }^{4} \mathrm{He}$ abundance ratio, and to monitor the synthesis and dispersal of heavy elements in a nearly pristine environment (Peimbert \& Torres-Peimbert 1974, Pagel et al. 1992, Izotov et al. 1997).

The understanding of the origin and implications of the starburst phenomenon in BCDs is necessary for elucidating evolutionary pathways of dwarf galaxies (DGs) in general. Are BCDs active phases in the lifetime of dormant dwarf irregulars (dIs) and do the latter fade to dwarf ellipticals (dEs) once their gas reservoir has been depleted (see e.g. Lin \& Faber 1983, Thuan 1985, Silk et al. 1987, Davies \& Phillipps 1988)? What is the role of the environment (e.g., Babul \& Rees 1992, Pustil'nik et al. 2001) and of Dark Matter (DM; Dekel \& Silk 1986, Ferrara \& Tolstoy 2000), and does the latter invariably dominate the mass within the Holmberg radius of a BCD (Papaderos et al. 1996b; hereafter P96b)? Do most BCDs undergo intermittent bursts or rather prolonged periods of elevated star formation (Vallenari \& Bomans 1996, Noguchi 2001, Rieschick \& Hensler 2001, Schulte-Ladbeck et al. 2001)? Despite much previous effort, the observational evidence available thus far is still too fragmentary to allow for unambiguous answers to the aforementioned questions.

Recent studies suggest, however, that key information for assessing DG evolution can be inferred from studies of the stellar low-surface brightness (LSB) host galaxy of these systems. In BCDs, the LSB component, underlying the SF regions, has first been disclosed through deep CCD imaging by Loose \& Thuan (1986, hereafter LT86), and has in the following been confirmed and further studied by various authors (cf., e.g., the list given later in this Section). This extended stellar host was found to account for $\sim 1 / 2$ of the light inside the $25 B$ mag/ $\square$ " isophote (P96b, Salzer \& Norton 1999), and to typically dominate the intensity and color distribution of BCDs for $\mu \gtrsim 24.5 \mathrm{~B}$ $\mathrm{mag} / \square^{\prime \prime}$. Such an evenly distributed, evolved stellar component is observed in all types of DGs, except for the extremely rare type of i0 BCDs in the classification scheme of LT86. Its red colors and smooth appearance in the main class of $\mathrm{iE} / \mathrm{nE}$ BCDs (LT86) indicate that these systems are several Gyr old, gas-rich DGs, having not been forming stars at the presently large rate throughout their lifetime. Different lines of evidence, 
outlined in the following, suggest that elaborate studies of the structural and kinematic properties of the LSB component are fundamental to assess at least two central issues of DG research: the evolutionary connections between DGs and the regulation of the SF process in these systems.

According to the standard evolutionary hypothesis for dwarf galaxies, dIs, dEs and BCDs differ basically by their gas content and the amplitude of their ongoing SF activity (Thuan 1985, Davies \& Phillipps 1988). One would therefore expect that, on average, the evolved stellar LSB host in all these three main DG classes is indistinguishable from one another with respect to its structural properties. However, P96b and subsequent authors (Patterson \& Thuan 1996, Marlowe et al. 1997, Salzer \& Norton 1999, see also Papaderos et al. 2002) found that, at equal absolute magnitude, the stellar LSB component of $\mathrm{iE} / \mathrm{nE}$ BCDs is systematically more compact than other types of DGs. P96b interpreted this structural disparity as the result of adiabatic contraction of the stellar LSB component of BCDs in response to a large-scale gas inflow, preceding the ignition of a starburst in a BCD. Quite interestingly, subsequent interferometric H I studies have shown that the gaseous halo of BCDs is by at least a factor of two more centrally concentrated than in dIs (van Zee et al. 1998,2001; see also Simpson et al. 2000), lending circumstantial support to the latter hypothesis. Whether or not such observational constraints can be accounted for by the dynamical evolution of the stellar and gaseous matter in DGs (P96b) or, alternatively, an extraordinarily dense Dark Matter halo, being particular to BCD galaxies (Meurer et al. 1998) awaits to be investigated by numerical 3D-simulations. These are also needed to explore a possible connection between the LSB morphology and the evolutionary state of BCDs, as proposed by Noeske et al. (2000).

The evolved LSB component contains, due to its high $M / L$, the bulk of the stellar matter in a BCD. Thus, provided that DM does not dominate within the optical radius, it forms, together with the gaseous component, the graviational potential within which SF activity occurs. It is therefore worth exploring whether the structural properties of the LSB component influence the SF process in a BCD. P96b found a trend for the fractional surface area of the SF component of BCDs to decrease with increasing LSB luminosity. For a constant $M / L$, this trend translates into a mass-morphology connection for BCDs: SF activity in more massive BCDs occurs mainly in the inner part of the LSB component, leading to a $\mathrm{nE}$ morphology and surface brightness profiles (SBPs) sometimes superficially resembling a de Vaucouleurs law. Conversely, SF activity in low-mass BCDs is spread over a larger portion of the LSB host, resulting in an $\mathrm{iE}$ morphology and SBPs possessing a conspicuous plateau feature (cf. Papaderos et al. 1996a, hereafter P96a) at intermediate intensity levels.

Our current knowledge of the nature of the underlying LSB component relies mainly on optical surface photometry studies of its faint periphery (e.g. LT86, Kunth et al. 1988, P96a, Telles et al. 1997, Marlowe et al. 1997, Doublier et al. 1997, 1999, Salzer \& Norton 1999, Vennik et al. 2000, Cairós et al. 2001a, 2001b, Makarova et al. 2002). In optical wavelengths, extended stellar and gaseous starburst emission overshines the LSB component out to a galactocentric radius of typically $\sim 2$ exponential scale lengths. Only at larger radii, where the starburst emission is in most cases negli- 
gible, the LSB host can be studied directly. However, to explore its possible dynamical influence on the global SF process, it is essential to pin down its intensity distribution at smaller radii, if possible just beneath the SF regions. Deprojection of SBPs would then allow one to put constraints on the density profile and the gravitational potential of the evolved stellar host (cf., e.g., P96a). In addition, one would be able to correct optical colors inside the SF component for the line-of-sight contribution of the underlying stellar background (cf. Cairós et al. 2002a, Papaderos et al. 2002), thus better constrain the SF history of these systems.

One way to alleviate the problem of the extended starburst emission is to extend studies to the Near Infrared (NIR). At these wavelengths, the young stellar populations and the ionized gas contribute a smaller fraction of the total light of the galaxy than in the optical. For instance, evolutionary synthesis models by Krüger et al. (1995) predict that a moderately strong burst during its peak luminosity accounts for only 20\% of a BCD's emission in the $K$, but for $\gtrsim 80 \%$ in the $B$ band. Observations of BCDs also show that starburst emission is weaker in the NIR, and becomes negligible at a smaller galactocentric distance than in the optical (e.g. Vanzi et al. 1996, 2002; Beck et al. 1997; Alton et al. 1994; James 1994). NIR data allow therefore to study the stellar LSB component at smaller radii and, using optical-NIR colors (e.g. $B-J$ ), better constrain its formation history. To achieve these objectives one needs to unambiguously detect the LSB component and study its intensity over a sufficient span ( $\Delta \mu \gtrsim 2$ mag). Empirical estimates, based on published optical and NIR data, suggest that these requirements are met by extending NIR studies to $\sim 22-24 J \mathrm{mag} / \square^{\prime \prime}$. So far, only a few BCDs have been studied at those surface brightness levels with an accuracy high enough to pinpoint structural properties and colors (Mkn 86, Gil de Paz et al. 2000a; Tol 0645-376, Doublier et al. 2001; Tol3, Vanzi et al. 2002).

The present analysis is the first part of an observational project (see also Cairós et al. 2002b; hereafter $\mathrm{C} 02 \mathrm{~b}$ ), aiming at a systematic study of the NIR properties of nearby BCDs with large array detectors on $4 \mathrm{~m}$-class telescopes. For this purpose, we take advantage of a large set of imaging data, homogeneous with respect to its limiting surface brightness and the methods used in its processing. The observations have been conducted so as to permit NIR surface photometry out to a comparable radius as in optical wavelengths, with the purpose of a multiwavelength investigation of the LSB component.

This paper is structured as follows: In Sect. 2, we describe the sample selection and data acquisition, and discuss the data reduction, photometric transformations and extinction corrections. Section 3 focusses on the derivation of SBPs and their decomposition into the luminosity components owing to the old LSB host and the younger stellar populations and SF regions. In Sect. 4, individual objects are presented in detail. Our results are discussed in Sect. 5. Sect. 6 summarizes this work. 


\section{I.2 Observations and data reduction}

\section{I.2.1 Sample selection}

Our sample covers the whole morphological spectrum of BCDs, containing both examples of the main morphological class (iE/nE systems according to LT86), and of the less frequent iI/iI,C/i0 BCD classes. The latter subset includes the metal-poor galaxies Tol 65 and Tol 1214-277 $\left(Z<Z_{\odot} / 20\right)$. The sample also includes one intrinsically luminous blue compact galaxy (BCG), UM 448.

Table I. 1 lists the adopted distance to each BCD. Whenever available, literature distances based on standard candles were preferred for nearby objects. Those literature distances that rely on redshifts have been corrected for peculiar motions within the local supercluster and assume a Hubble constant $H_{0}=75 \mathrm{~km} \mathrm{~s}^{-1} \mathrm{Mpc}^{-1}$. When no literature data was available, the distance was inferred from the $H_{0}$ and the heliocentric velocity listed in the NASA Extragalactic Database (NED). The latter was first transformed to the velocity relative to the local group (LG) centroid using the NED velocity calculator, and subsequently corrected for a LG infall towards the Virgo Cluster center $\left(l=284^{\circ}, b=74^{\circ}\right)$ at $200 \mathrm{~km} \mathrm{~s}^{-1}$ (Tammann \& Sandage 1985) .

\section{I.2.2 Data acquisition}

The NIR images were observed with the ESO 3.6m NTT telescope, La Silla/Chile, during three consecutive nights from April 21st to 24th, 2000. The seeing ranged from 0 ". 4 to $1.0 \mathrm{FWHM}$ and the transparency variations were $\lesssim 1 \%$ during the first and third night, and $\lesssim 2 \%$ during the second night. Additional NIR images were taken at the 3.6m telescope of the German-Spanish Astronomical Center, Calar Alto, Spain, during several observing runs. The seeing conditions were generally average (December 26th, 1999: FWHM 1".3 - 3".5, transparency fair; May 10th-15th, 2000: FWHM 0".8 1".4, transparency good to average; October 6th-10th, 2000: FWHM 1".2 - 2".0, transparency good to fair). Both cameras used, the SOFI at the NTT Nasmyth focus and the OMEGA PRIME at the Calar Alto 3.6m telescope's prime focus, were equipped with $1024 \times 1024$ pixel Rockwell HAWAII detectors. The pixel scales for SOFI, using the large field objective, and OMEGA PRIME were 0'.292 and 0!'396, yielding a FOV of 4.94 and 6.76, respectively. The data was taken through the $J$ and $H$ broad band filters, as well as the modified $K$ filters $K_{s}$ at the NTT and $K^{\prime}$ at Calar Alto, both selected to extenuate the contribution of thermal background.

To achieve an adequate sampling of the background variations, the telescope was offset between exposures typically each 60 seconds. While compact galaxies were jittered within the FOV, larger objects required to alternately observe the object and the sky. To avoid detector saturation, images were obtained by summing up subexposures of few seconds each. The total on-object exposure times, after rejection of subexposures affected by unstable readout electronics or strong background gradients, are listed in Tab. I.1. 
Table I.1. Sample galaxies

\begin{tabular}{|c|c|c|c|c|c|c|c|c|c|}
\hline Object & $\begin{array}{l}\text { RA(J2000) } \\
\text { DEC(J2000) } \\
\text { (2) }\end{array}$ & $\begin{array}{c}\mathrm{t}_{J, \mathrm{NTT}} \\
\mathrm{t}_{J, \mathrm{CA}} \\
{[\mathrm{s}]} \\
(3)\end{array}$ & $\begin{array}{c}\mathrm{t}_{H, \mathrm{NTT}} \\
\mathrm{t}_{H, \mathrm{CA}} \\
{[\mathrm{s}]} \\
(4)\end{array}$ & $\begin{array}{c}\mathrm{t}_{K_{s}, \mathrm{NTT}} \\
\mathrm{t}_{K^{\prime}, \mathrm{CA}} \\
{[\mathrm{s}]} \\
(5)\end{array}$ & $\begin{array}{c}\mathrm{M}_{B} \\
\text { (ref.) } \\
{[\mathrm{mag}]} \\
(6)\end{array}$ & $\begin{array}{c}\mathrm{A}_{B} \\
{[\mathrm{mag}]} \\
(7)\end{array}$ & $\begin{array}{c}D \\
\text { (ref.) } \\
{[\mathrm{Mpc}]} \\
(8)\end{array}$ & $\begin{array}{c}\text { FWHM } \\
\text { (final) } \\
{\left[^{\prime \prime}\right]} \\
(9)\end{array}$ & $\begin{array}{l}\text { other } \\
\text { names }\end{array}$ \\
\hline $\begin{array}{l}\text { Tol } 3 \\
\text { (iE) }\end{array}$ & $\begin{array}{r}10^{\mathrm{h}} 06^{\mathrm{m}} 33^{\mathrm{s}} .6 \\
-29^{\circ} 56^{\prime} 09^{\prime \prime} .0\end{array}$ & $\begin{array}{c}1440 \\
-\end{array}$ & $\begin{array}{c}1560 \\
-\end{array}$ & $\begin{array}{c}2100 \\
-\end{array}$ & $\begin{array}{c}-18.0 \\
\text { (a) }\end{array}$ & 0.33 & $\begin{array}{l}13.8 \\
\text { (a) }\end{array}$ & 1.2 & $\begin{array}{l}\text { NGC 3125; ESO 435-G041; } \\
\text { Tol1004-296 }\end{array}$ \\
\hline $\begin{array}{l}\text { Haro 14 } \\
(\mathrm{iE} / \mathrm{nE})\end{array}$ & $\begin{array}{r}00^{\mathrm{h}} 45^{\mathrm{m}} 46^{\mathrm{s}} .4 \\
-15^{\circ} 35^{\prime} 49^{\prime \prime} .0\end{array}$ & $\overline{-}$ & $\overline{1680}$ & $\overline{1920}$ & $\begin{array}{c}-17.0 \\
\text { (a) }\end{array}$ & 0.09 & $\begin{array}{l}12.5 \\
\text { (a) }\end{array}$ & 1.6 & $\begin{array}{l}\text { NGC 0244; UGCA 010; } \\
\text { VV } 728\end{array}$ \\
\hline $\begin{array}{l}\text { UM } 461 \\
\text { (iI) }\end{array}$ & $\begin{array}{r}11^{\mathrm{h}} 51^{\mathrm{m}} 33^{\mathrm{s}} .0 \\
-02^{\circ} 22^{\prime} 23^{\prime \prime} .0\end{array}$ & $\begin{array}{c}1500 \\
-\end{array}$ & $\begin{array}{c}1500 \\
-\end{array}$ & $\begin{array}{c}2100 \\
-\end{array}$ & $\begin{array}{c}-14.9 \\
(j)\end{array}$ & 0.08 & $\begin{array}{c}14.3 \\
\text { (e) }\end{array}$ & 0.7 & SCHG 1148-020 \\
\hline $\begin{array}{l}\text { He 2-10 } \\
(\mathrm{iE} / \mathrm{nE})\end{array}$ & $\begin{array}{r}08^{\mathrm{h}} 36^{\mathrm{m}} 15^{\mathrm{s}} .0 \\
-26^{\circ} 24^{\prime} 34^{\prime \prime} .0 \\
\end{array}$ & $\begin{array}{c}1140 \\
- \\
\end{array}$ & $\begin{array}{c}1500 \\
- \\
\end{array}$ & $\begin{array}{c}2040 \\
- \\
\end{array}$ & $\begin{array}{c}-18.7 \\
(\mathrm{k})\end{array}$ & 0.48 & $\begin{array}{l}8.7 \\
\text { (f) }\end{array}$ & 0.9 & ESO 495-G021 \\
\hline $\begin{array}{l}\text { Tol 1400-411 } \\
\text { (iI,C) }\end{array}$ & $\begin{array}{r}14^{\mathrm{h}} 03^{\mathrm{m}} 21^{\mathrm{s}} .0 \\
-41^{\circ} 22^{\prime} 44^{\prime \prime} .0\end{array}$ & $\begin{array}{c}1140 \\
-\end{array}$ & $\begin{array}{c}1500 \\
-\end{array}$ & $\begin{array}{c}2100 \\
-\end{array}$ & $\begin{array}{c}-16.5 \\
\text { (l) }\end{array}$ & 0.30 & $\begin{array}{l}4.8 \\
\text { (c) }\end{array}$ & 0.9 & $\begin{array}{l}\text { NGC 5408; Tol 116; } \\
\text { ESO 325-G047 }\end{array}$ \\
\hline $\begin{array}{l}\text { Pox 4/Pox 4B } \\
\text { (iI + comp.) }\end{array}$ & $\begin{array}{r}11^{\mathrm{h}} 51^{\mathrm{m}} 11^{\mathrm{s}} .6 \\
-20^{\circ} 36^{\prime} 02^{\prime \prime} .0 \\
\end{array}$ & $\begin{array}{c}1500 \\
- \\
\end{array}$ & $\begin{array}{c}1500 \\
- \\
\end{array}$ & $\begin{array}{c}2100 \\
- \\
\end{array}$ & $\begin{array}{c}-18.8 \\
(\mathrm{~m})\end{array}$ & 0.17 & $\begin{array}{l}46.7 \\
(\mathrm{~b})\end{array}$ & 0.6 & CAM 1148-2020 \\
\hline $\begin{array}{l}\text { Tol 65 } \\
(\mathrm{i} 0 / \mathrm{iI}, \mathrm{C})\end{array}$ & $\begin{array}{c}12^{\mathrm{h}} 25^{\mathrm{m}} 46^{\mathrm{s}} .9 \\
-36^{\circ} 14^{\prime} 01^{\prime \prime} .0 \mathrm{~s}\end{array}$ & $\begin{array}{c}2100 \\
-\end{array}$ & $\begin{array}{c}2100 \\
-\end{array}$ & $\begin{array}{c}3000 \\
-\end{array}$ & $\begin{array}{c}-15.3 \\
\text { (n) }\end{array}$ & 0.32 & $\begin{array}{c}34.2 \\
(\mathrm{~b})\end{array}$ & 0.6 & $\begin{array}{l}\text { ESO 380-G027; } \\
\text { Tol 1223-359 }\end{array}$ \\
\hline $\begin{array}{l}\text { Tol 1214-277 } \\
(\mathrm{i} / \mathrm{iI}, \mathrm{C}) \\
\end{array}$ & $\begin{array}{r}12^{\mathrm{h}} 17^{\mathrm{m}} 17^{\mathrm{s}} .1 \\
-28^{\circ} 02^{\prime} 33^{\prime \prime} .0 \\
\end{array}$ & $\begin{array}{c}1200 \\
- \\
\end{array}$ & $\begin{array}{c}1500 \\
- \\
\end{array}$ & $\begin{array}{c}2100 \\
- \\
\end{array}$ & $\begin{array}{c}-16.9 \\
\text { (o) } \\
\end{array}$ & 0.28 & $\begin{array}{c}102.6 \\
\text { (b) } \\
\end{array}$ & 0.9 & $\begin{array}{l}\text { SCHG 1214-277; } \\
\text { Tol } 21\end{array}$ \\
\hline
\end{tabular}


Table I.1. (continued)

\begin{tabular}{|c|c|c|c|c|c|c|c|c|c|}
\hline 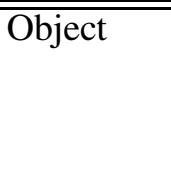 & $\begin{array}{l}\text { RA(J2000) } \\
\text { DEC(J2000) } \\
\text { (2) }\end{array}$ & $\begin{array}{c}\mathrm{t}_{J, \mathrm{NTT}} \\
\mathrm{t}_{J, \mathrm{CA}} \\
{[\mathrm{s}]} \\
(3)\end{array}$ & $\begin{array}{c}\mathrm{t}_{H, \mathrm{NTT}} \\
\mathrm{t}_{H, \mathrm{CA}} \\
{[\mathrm{s}]} \\
(4)\end{array}$ & $\begin{array}{c}\mathrm{t}_{K_{s}, \mathrm{NTT}} \\
\mathrm{t}_{K^{\prime}, \mathrm{CA}} \\
{[\mathrm{s}]} \\
(5)\end{array}$ & $\begin{array}{c}\mathrm{M}_{B} \\
\text { (ref.) } \\
{[\mathrm{mag}]} \\
(6)\end{array}$ & $\begin{array}{c}\mathrm{A}_{B} \\
{[\mathrm{mag}]} \\
(7)\end{array}$ & $\begin{array}{c}D \\
\text { (ref.) } \\
{[\mathrm{Mpc}]} \\
(8)\end{array}$ & $\begin{array}{c}\text { FWHM } \\
\text { (final)" } \\
{\left[{ }^{\prime \prime}\right]} \\
(9)\end{array}$ & $\begin{array}{l}\begin{array}{l}\text { other } \\
\text { names }\end{array} \\
(10)\end{array}$ \\
\hline $\begin{array}{l}\text { Mkn } 178 \\
\text { (iE) }\end{array}$ & $\begin{array}{r}11^{\mathrm{h}} 33^{\mathrm{m}} 29^{\mathrm{s}} .1 \\
+49^{\circ} 14^{\prime} 17^{\prime \prime} .0\end{array}$ & $\begin{array}{c}240 \\
-\end{array}$ & $\begin{array}{c}1380 \\
-\end{array}$ & $\begin{array}{c}1380 \\
-\end{array}$ & $\begin{array}{c}-13.9 \\
(\mathrm{p})\end{array}$ & 0.08 & $\begin{array}{l}4.2 \\
(\mathrm{~h})\end{array}$ & 1.6 & $\begin{array}{l}\text { UGC 06541; HOLN } \\
\text { CGCG 242-046; SB }\end{array}$ \\
\hline $\begin{array}{l}\text { Mkn } 1329 \\
(\text { iI,C) }\end{array}$ & $\begin{array}{r}12^{\mathrm{h}} 37^{\mathrm{m}} 03^{\mathrm{s}} .0 \\
+06^{\circ} 55^{\prime} 36^{\prime \prime} .0\end{array}$ & $\begin{array}{c}300 \\
1200\end{array}$ & $\begin{array}{l}360 \\
360\end{array}$ & $\begin{array}{l}480 \\
960\end{array}$ & $\begin{array}{c}-16.8 \\
\text { (i) }\end{array}$ & 0.10 & $\begin{array}{l}16.0 \\
\text { (i) }\end{array}$ & 0.7 & $\begin{array}{l}\text { VCC 1699; IC 3589/91; } \\
\text { UGC } 7790\end{array}$ \\
\hline $\begin{array}{l}\text { IC } 4662 \\
(\mathrm{dI} / \mathrm{iE})\end{array}$ & $\begin{array}{r}17^{\mathrm{h}} 47^{\mathrm{m}} 06^{\mathrm{s}} .4 \\
-64^{\circ} 38^{\prime} 25^{\prime \prime} .0\end{array}$ & $\begin{array}{c}1980 \\
-\end{array}$ & $\begin{array}{c}1620 \\
-\end{array}$ & $\begin{array}{c}660 \\
-\end{array}$ & $\begin{array}{c}-14.9 \\
(1)\end{array}$ & 0.30 & $\begin{array}{l}2.0 \\
(\mathrm{~g})\end{array}$ & 0.8 & $\begin{array}{l}\text { ESO 102-G014; } \\
\text { He 2-269 }\end{array}$ \\
\hline $\begin{array}{l}\mathrm{UM} 448 \\
\text { (iI/BCG) }\end{array}$ & $\begin{array}{r}11^{\mathrm{h}} 42^{\mathrm{m}} 12^{\mathrm{s}} .4 \\
+00^{\circ} 20^{\prime} 03^{\prime \prime} .0 \\
\end{array}$ & $\begin{array}{l}300 \\
840\end{array}$ & $\begin{array}{l}360 \\
900\end{array}$ & $\begin{array}{l}480 \\
1200\end{array}$ & $\begin{array}{c}-19.8 \\
(1)\end{array}$ & 0.11 & $\begin{array}{c}76.1 \\
(\mathrm{~d})\end{array}$ & 0.7 & $\begin{array}{l}\text { UGC 06665; SCHG 1139+006; } \\
\text { Ark 312; ARP 161; Mkn 1304 }\end{array}$ \\
\hline
\end{tabular}

\$: Resolution of the best image set available for the respective galaxy, after reduction and combination (a): Marlowe et al. (1999), (b): inferred from the heliocentric velocity $v_{\text {hel }}$ listed in the NED, corrected for solar motion with respect to the center of the Virgo Cluster and adopting $\mathrm{H}_{0}=75 \mathrm{~km} \mathrm{~s}^{-1} \mathrm{Mpc}^{-1}$ (cf. Sect. I.2.1). (c): Karachentsev et al. (2002), (d): Mirabel \& Sanders 1988, (e): $v_{L G}$ from Smoker et al. (2000), corrected for Local Group infall to the Virgo Cluster like b); (f): Tully (1988), (g): Heydari-Malayeri 1990, (h): Schulte-Ladbeck et al. (2000), (i): Yasuda et al. (1997), (j): $\mathrm{m}_{B}$ from Méndez \& Esteban (2000), (k): $\mathrm{m}_{B}$ from Papaderos (1998), (1): $\mathrm{m}_{B}$ from the RC3 (de Vaucouleurs et al. 1991), (m): $\mathrm{m}_{B}$ for Pox 4 from Méndez \& Esteban (1999); no published $\mathrm{m}_{B}$ for Pox 4B (n): $\mathrm{m}_{B}$ from P99, (o): $\mathrm{m}_{B}$ from F01, (p): $\mathrm{m}_{B}$ from Papaderos et al. (2002) 


\section{I.2.3 Data reduction}

The exposures have been processed semi-interactively, employing our NIR image reduction software based on ESO MIDAS ${ }^{3}$. The procedures used for basic reduction, background subtraction, image alignment and coaddition follow the recipes which are detailed in, e.g., the SOFI User Manual ${ }^{4}$.

Since surface photometry in the LSB regime depends sensitively on the quality of the background subtraction, care has been exercised to eliminate residuals of this correction on both, small and large spatial scales. For this purpose we have implemented a number of additional corrective steps into our software package. For each single science frame of an exposure sequence we first computed an individual master background image, using subexposures that were taken closest in time, but at a sufficiently large distance from the target. Prior to the calculation of the master background frame, the input background images were cleaned from bright contaminating sources and normalized to the same mean intensity (see the SOFI User Manual for details). The master background frame computed this way was in turn scaled to the background level of the respective science frame, and subtracted. Each of the resulting subexposures was subsequently checked for a possible residual background gradient and, whenever necessary, rectified using a first order polynomial fit. The result image was obtained through combination of all background-corrected and coregistered subexposures. In this procedure, out of all pixels with identical sky coordinates, only those at sound detector coordinates have been included in the calculations.

In rare cases, background variations in the final images needed to be interactively corrected by fitting twodimensional polynomials. Alternatively, regions still affected by small-scale residuals in the background subtraction were extracted as subimages, corrected in the latter manner, and inserted back into the original frame. Likewise, bleeding artifacts, caused by bright sources in the NTT/SOFI FOV, or blooming of bright, overexposed foreground stars in the very outskirts of IC 4662 (northern edge) and Tol 1400-411 (east and west) were modeled and subtracted out. SBPs of the latter two galaxies were only analyzed well above intensity levels where slight residuals from the replaced bright stars could possibly contribute.

Final images taken in the same filter during different nights or at different telescopes were aligned to each other, transformed to equal pixel scales and resolutions, and coadded after weighting each one by its $(S / N)^{2}$. The FWHM of the result images is listed in Tab. I.1. Images used for aperture or surface photometry were manually cleaned for fore- and background sources.

\section{I.2.4 Flux Calibration}

The SOFI data was calibrated by observing at different airmasses standard stars from Persson et al. (1998) six times each night. The excellent photometric stability throughout the NTT observing run has allowed for the derivation of an airmass-dependent

\footnotetext{
${ }^{3}$ Munich Image data Analysis System, provided by the European Southern Observatory (ESO).

${ }^{4}$ Issue 1.2, available online at http: / / www . eso.org
} 
calibration with a scatter of $\lesssim 0.01$ mag during nights 1 and 3 and $\lesssim 0.02$ mag during night 2 . Zero points and airmass-dependent calibration coefficients both agree well with the average values supplied by the NTT/SOFI support team. Images of UM448 and Mkn 1329, obtained by combining Calar Alto and NTT data, were calibrated using aperture photometry of bright stars in the SOFI FOV. Despite different $K$ filters used at those telescopes, the integral $K_{s}$ fluxes are essentially preserved, as color terms in the filter transformations (Eqs. I.3) are not exceeding a few $0.01 \mathrm{mag}$.

The BCDs Haro 14 and Mkn 178, for which no NTT observations are available, were calibrated using the Two Micron All Sky Survey (2MASS) catalogue ${ }^{5}$ (Cutri et al. 2000, Jarrett et al. 2000). As 2MASS data (cf. Andreon 2002) may, due to their limited sensitivity, slightly underestimate the flux within the extended LSB component, we computed calibration terms using 2MASS field stars in the close vicinity of Haro 14 and Mkn 178.

\section{I.2.4.1 Transformation to other NIR photometric systems}

Unless stated otherwise, all magnitudes and colors given in this paper refer to the calibrations described in the previous section, and to the photometric systems defined by the instrumental setup of each telescope (see Sect. I.2). However, whenever photometric quantities of the sample BCDs are compared either among each other, or with model predictions and data from the literature, they are first transformed to the 2MASS photometric system, using the relations described below.

The calibration obtained at the NTT with the SOFI instrument is based on standards by Persson et al. (1998), and can therefore be transformed to the Persson et al. (1998) Las Campanas Observatory (LCO) system using the color transformations given by the SOFI user manual (Issue 1.3, 16/08/2000). A transformation from the latter system to the 2MASS system is described by Carpenter (2001). Combination of both latter transformations yields the relations:

$$
\begin{gathered}
K_{s, 2 \mathrm{M}}=K_{s, \mathrm{~S}}+0.021(J-K)-0.010 \\
(J-H)_{2 \mathrm{M}}=0.995(J-H)_{\mathrm{S}}+0.015(J-K)+0.002 \\
\left(H-K_{s}\right)_{2 \mathrm{M}}=1.029\left(H-K_{s}\right)_{\mathrm{S}}-0.046(J-K)+0.005,
\end{gathered}
$$

where the indices " $2 \mathrm{M}$ " and "S" denote the 2MASS and SOFI systems, respectively. Cumulative uncertainties introduced by this transformation are $\lesssim 0.01$ mag for $K_{s}$ and $\lesssim 0.015 \mathrm{mag}$ for $(J-H)$ and $\left(H-K_{s}\right)$, given the typical range of values expected for NIR colors of BCDs.

No transformations to other standard NIR systems are available for the photometric system defined by the Calar Alto 3.6m telescope and the OMEGA PRIME camera. As stated in Sect. I.2.4, the calibration of the Calar Alto frames is tied to the 2MASS

\footnotetext{
5 http://www.ipac.caltech.edu/2mass /
} 
zero points. The remaining uncertainties introduced by the unknown color terms can be estimated from Carpenter (2001) to be

$$
\mid(\text { color })_{2 M}-(\text { color })_{\Omega^{\prime}}|\lesssim| 0.08(\text { color })_{\Omega^{\prime}} \mid
$$

per transformed color, i.e., the approximate upper limit of the color terms entering into the transformation between standard NIR photometric systems.

The $K^{\prime}$ and $K_{s}$ magnitudes are related by the following equations (cf. Wainscoat \& Cowie 1992 and the SOFI user manual):

$$
\begin{gathered}
K_{s}=K+0.005(J-K) \\
K=K^{\prime}-(0.22 \pm 0.03)(H-K)
\end{gathered}
$$

\section{I.2.4.2 Extinction correction:}

Magnitudes and colors given in this paper are corrected for Galactic extinction, adopting values derived from the $B$ band extinction maps by Schlegel et al. (1998) (cf. Tab. I.1) and the standard $\left(\mathrm{R}_{V}=3.1\right)$ extinction law (Cardelli et al. 1989) implemented into the NED. No attempt was made to correct for internal extinction, since this is known to vary spatially even in the most metal-deficient BCDs (cf., e.g., Guseva et al. 2001, Cannon et al. 2002, Hunt et al. 2003) and can be reliably constrained in the SF regions only.

\section{I.3 Data analysis and SBP derivation}

\section{I.3.1 Derivation of surface brightness and color profiles}

Surface photometry aims at a standardized one-dimensional representation of a galaxy's two-dimensional flux pattern. One technique to compute surface brightness profiles (SBPs) requires the determination of the size $A(\mu)$ of the galaxy in $\square^{\prime \prime}$ for a series of surface brightness levels $\mu$ (mag/ $\left.\square^{\prime \prime}\right)$. By this definition, the equivalent radius $R^{*}=\sqrt{(A(\mu) / \pi)}$ is a monotonic function of the surface brightness $\mu$ and vice versa. In order to derive SBPs this way, one has to keep track of the morphology and angular extent of a BCD throughout its intensity span, i.e. in general to be able to interpolate an isophote down to the faintest measured level $\mu$ of an SBP. By this condition one can visually check for and screen-out fore- or background sources in the periphery of the galaxy, thus make sure that source confusion does not affect SBPs at faint levels. This task is more difficult to achieve when computing SBPs employing photon statistics inside circular or elliptical annuli, extending out to a user-defined maximal radius $r_{\text {max }}$. SBPs derived for an irregular system by such techniques may vary from case to case, depending on, e.g., the adopted $r_{\max }$ or "center" of the galaxy. It should be borne in mind that techniques of this kind, when applied to galaxies with a morphology significantly departing from the assumed circular symmetry, can strongly overestimate the 
exponential scale length and underestimate the central intensity of the LSB component (cf., e.g., Marlowe et al. 1997).

In the present analysis, we compute SBPs using method iv), described in Papaderos et al. (2002). This is a hybrid technique, incorporating features of both aforementioned approaches (determination of the $R^{*}$ corresponding to a user-defined $\mu$, as opposed to the determination of the mean surface brightness $\bar{\mu}$ inside a circular annulus with a user-defined radius $R^{*} \pm \Delta R^{*}$ ).

For a set of $n$ intensity intervals $I_{n}$, with a mean intensity decreasing as $n$ increases, masks $M_{n}$ are generated, each of which extracts from a smoothed image of the galaxy the areas with intensities within $I_{n}$. The equivalent radius $R_{n}^{\star}$ corresponding to the mask area $A_{n}$ is:

$$
R_{n}^{\star}=\left(\frac{1}{\pi}\left[\sum_{i=1}^{n-1} A_{i}+\frac{1}{2} A_{n}\right]\right)^{\frac{1}{2}}
$$

The surface brightness $\mu\left(R_{n}^{\star}\right)$ is calculated from the mean intensity of the original image within the area $M_{n}$. Method iv) overcomes the problem of the artificial SBP flattening for low $S / N$ levels (see discussion in e.g. Noeske 1999, Noeske et al. 2000 and Cairós 2000) and proves reliable down to very faint surface brightness levels $\left(I_{n}<\right.$ sky noise).

As a check for consistency, we also derived SBPs through ellipse fitting to isophotes or methods ii) and iii) in P96a, applying in all cases a minimum of image filtering to moderate photon noise. The resulting SBPs have been compared with deep optical surface photometry to ensure that the underlying LSB component has been detected and modelled (see Sect. I.3.2) over a sufficient radius range.

Color profiles were derived by subtracting the SBPs from each other, after the latter have been smoothed to equal FWHM. Surface brightness and color profiles, corrected for Galactic extinction (Sect. I.2.4.2), are shown in Figs. I.2 through I.14.

\section{I.3.2 Profile Decomposition}

The stellar emission of a BCD is due to the superposition of two distinct populations with respect to their $\mathcal{M} / \mathcal{L}$ ratio and spatial extent: (i) the underlying LSB host galaxy which, owing to its high $\mathcal{M} / \mathcal{L}$, contains the bulk of the system's stellar mass, and (ii) the younger stellar population, attributable to the ongoing and recent SF activity. In the main class of $\mathrm{iE} / \mathrm{nE} \mathrm{BCDs}$, the younger stellar component dominates the optical emission inside $\sim 2$ exponential scale lengths of the underlying LSB host and contributes, together with ionized gas emission, $\sim 1 / 2$ of the $B$ light within the $25 B$ mag/ $\square$ " isophote (P96b, Noeske 1999, Salzer \& Norton 1999). Evidently, a meaningful study of the structural properties of a BCD requires the decomposition of SBPs into these two main photometric components. In the following, we employ a simple SBP decomposition scheme, in which we fit only the LSB emission. Subtraction of the best fit LSB model from the SBP allows us to deduce the luminosity fraction and spatial extent of the superimposed SF component. In order to make sure that extended starburst emission does not affect the decomposition results, we fitted the LSB component 
Table I.2. Structural properties of the $\operatorname{dwarfs}^{a}$; see also the discussion of individual objects

\begin{tabular}{|c|c|c|c|c|c|c|c|c|c|c|c|c|}
\hline $\begin{array}{l}\text { Name } \\
(b, q)^{b} \\
\eta_{\mathrm{LSB}}^{c} \\
(1)\end{array}$ & Band & $\begin{array}{c}\mu_{E, 0} \\
\mathrm{mag} / \square^{\prime \prime}\end{array}$ & $\begin{array}{c}\alpha \\
\mathrm{kpc}\end{array}$ & $\begin{array}{c}m_{\mathrm{LSB}}^{\mathrm{fit}} \\
\mathrm{mag}\end{array}$ & $\begin{array}{l}P_{\text {iso }} \\
\mathrm{kpc}\end{array}$ & $\begin{array}{c}m_{P_{\text {iso }}} \\
\text { mag }\end{array}$ & $\begin{array}{l}E_{\text {iso }} \\
\mathrm{kpc}\end{array}$ & $\begin{array}{c}m_{\mathrm{E}_{\text {iso }}} \\
\text { mag }\end{array}$ & $\begin{array}{c}m_{\mathrm{SBP}} \\
\text { mag }\end{array}$ & $\begin{array}{c}m_{\text {tot }} \\
\text { mag }\end{array}$ & $\begin{array}{c}r_{\mathrm{eff}}, r_{80} \\
\mathrm{kpc}\end{array}$ & (13) \\
\hline Tol $3^{e}$ & $J$ & $18.24 \pm 0.05$ & $0.52 \pm 0.01$ & 11.78 & 1.20 & 12.42 & 2.29 & 11.86 & 11.31 & 11.29 & $0.61,1.23$ & 1.45 \\
\hline \multirow[t]{2}{*}{$\star$} & $H$ & $17.74 \pm 0.06$ & $0.53 \pm 0.01$ & 11.26 & 1.18 & 11.76 & 2.07 & 11.37 & 10.73 & 10.69 & $0.61,1.22$ & 1.43 \\
\hline & $K_{s}$ & $17.45 \pm 0.09$ & $0.51 \pm 0.01$ & 11.06 & 1.18 & 11.48 & 2.12 & 11.15 & 10.50 & 10.46 & $0.57,1.15$ & 1.49 \\
\hline Haro $14^{f}$ & $\mathrm{~J}$ & $17.46 \pm 0.12$ & $0.37 \pm 0.01$ & 12.70 & 1.39 & 12.45 & 1.88 & 12.82 & 11.81 & 11.78 & $0.61,1.21$ & 1.18 \\
\hline $3.6,0.94$ & $\mathrm{H}$ & $16.97 \pm 0.26$ & $0.39 \pm 0.02$ & 12.11 & 1.37 & 11.69 & 1.76 & 12.31 & 11.12 & 11.07 & $0.61,1.22$ & 1.25 \\
\hline 0.49 & $\mathrm{~K}^{\prime}$ & $16.88 \pm 0.94$ & $0.39 \pm 0.08$ & 11.99 & 1.32 & 11.54 & 1.83 & 12.17 & 10.98 & 10.97 & $0.60,1.21$ & 1.45 \\
\hline $\mathrm{UM} 461^{e}$ & $\mathrm{~J}$ & $19.37 \pm 0.18$ & $0.21 \pm 0.01$ & 15.56 & 0.42 & 16.19 & 0.69 & 15.88 & 15.09 & 15.04 & $0.34,0.57$ & 1.96 \\
\hline $2.3,0.85$ & $\mathrm{H}$ & $18.82 \pm 0.25$ & $0.21 \pm 0.02$ & 14.98 & 0.35 & 16.12 & 0.60 & 15.46 & 14.66 & 14.57 & $0.37,0.61$ & 1.82 \\
\hline 0.49 & $\mathrm{~K}_{s}$ & $18.47 \pm 0.79$ & $0.19 \pm 0.04$ & 14.79 & 0.36 & 15.75 & 0.63 & 15.14 & 14.46 & 14.37 & $0.32,0.54$ & 2.04 \\
\hline Henize $2-10^{e}$ & $\mathrm{~J}$ & $18.46 \pm 0.03$ & $0.67 \pm 0.01$ & 10.46 & 0.87 & 10.57 & 2.80 & 10.55 & 9.80 & 9.70 & $0.41,1.26$ & 2.50 \\
\hline \multirow[t]{2}{*}{$\star$} & $\mathrm{H}$ & $17.75 \pm 0.04$ & $0.64 \pm 0.01$ & 9.85 & 0.78 & 9.94 & 2.51 & 9.96 & 9.17 & 9.13 & $0.40,1.22$ & 2.44 \\
\hline & $\mathrm{K}_{s}$ & $17.51 \pm 0.03$ & $0.62 \pm 0.01$ & 9.68 & 0.79 & 9.62 & 2.56 & 9.78 & 8.95 & 8.89 & $0.34,1.04$ & 2.44 \\
\hline Tol $1400-411^{e}$ & $\bar{J}$ & $18.29 \pm 0.11$ & $0.33 \pm 0.01$ & 11.18 & 0.69 & 12.67 & 1.41 & 11.32 & 10.99 & 10.91 & $0.60,0.98$ & 2.04 \\
\hline $3.0,0.82$ & $\mathrm{H}^{d}$ & $17.30 \pm 0.14$ & $0.27 \pm 0.01$ & 10.57 & 0.31 & 13.65 & 1.18 & 10.71 & 10.57 & 10.55 & $0.57,0.93$ & 1.33 \\
\hline 0.63 & $\mathrm{~K}_{s}$ & $17.39 \pm 0.09$ & $0.30 \pm 0.01$ & 10.43 & 0.50 & 12.49 & 1.28 & 10.58 & 10.32 & 10.31 & $0.59,0.97$ & 1.75 \\
\hline Pox $4^{e}$ & $\bar{J}$ & $19.26 \pm 0.15$ & $0.86 \pm 0.03$ & 15.15 & 2.27 & 14.80 & 2.87 & 15.54 & 14.24 & 14.20 & $1.24,2.17$ & 1.75 \\
\hline $2.7,0.90$ & $\mathrm{H}^{d}$ & $19.10 \pm 0.31$ & $0.88 \pm 0.07$ & 14.95 & 2.18 & 14.35 & 1.79 & 16.10 & 13.85 & 13.81 & $1.27,2.15$ & 1.45 \\
\hline 0.42 & $\mathrm{~K}_{s}{ }^{d}$ & - & - & - & - & - & - & - & 13.54 & 13.52 & $1.18,2.09$ & 1.96 \\
\hline $\operatorname{Pox} 4 \mathrm{~B}^{e}$ & $\bar{J}$ & $19.47 \pm 0.40$ & $0.29 \pm 0.04$ & 17.37 & 0.51 & 19.29 & 0.93 & 17.67 & 17.21 & 17.12 & $0.54,0.87$ & 0.75 \\
\hline $2.0,0.80$ & $\mathrm{H}^{d}$ & - & - & - & - & - & - & - & 16.83 & 16.72 & $0.51,0.80$ & 0.77 \\
\hline 0.49 & $\mathrm{~K}_{s}{ }^{d}$ & - & - & - & - & - & - & - & 16.75 & 16.52 & $0.47,0.69$ & 0.75 \\
\hline
\end{tabular}


Table I.2. (continued)

\begin{tabular}{|c|c|c|c|c|c|c|c|c|c|c|c|c|}
\hline $\begin{array}{l}\text { Name } \\
(b, q)^{b} \\
\eta_{\mathrm{LSB}}^{c} \\
(1)\end{array}$ & Band & $\begin{array}{c}\mu_{E, 0} \\
\mathrm{mag} / \square^{\prime \prime}\end{array}$ & $\begin{array}{c}\alpha \\
\mathrm{kpc}\end{array}$ & $\begin{array}{c}m_{\mathrm{LSB}}^{\mathrm{fit}} \\
\text { mag }\end{array}$ & $\begin{array}{l}P_{\text {iso }} \\
\text { kpc }\end{array}$ & $\begin{array}{c}m_{P_{\text {iso }}} \\
\text { mag }\end{array}$ & $\begin{array}{l}E_{\text {iso }} \\
\mathrm{kpc}\end{array}$ & $\begin{array}{c}m_{\mathrm{E}_{\text {iso }}} \\
\text { mag }\end{array}$ & $\begin{array}{c}m_{\mathrm{SBP}} \\
\text { mag }\end{array}$ & $\begin{array}{c}m_{\text {tot }} \\
\text { mag }\end{array}$ & $\begin{array}{c}r_{\text {eff }}, r_{80} \\
\mathrm{kpc}\end{array}$ & (13) \\
\hline Tol $65^{e}$ & $J$ & $19.58 \pm 0.18$ & $0.28 \pm 0.02$ & 17.25 & 0.43 & 17.47 & 0.82 & 17.79 & 16.57 & 16.55 & $0.37,0.80$ & 1.59 \\
\hline $2.7,0.90$ & $H^{d}$ & - & - & - & - & - & - & - & 16.23 & 16.30 & $0.42,0.84$ & - \\
\hline 0.46 & $K_{s}{ }^{d}$ & 一 & - & 一 & 一 & 一 & - & - & 16.03 & 15.99 & $0.24,0.61$ & 一 \\
\hline Tol 1214-277 $7^{e, g}$ & $\mathbf{J}^{d}$ & $19.16 \pm 0.24$ & $0.53 \pm 0.03$ & 18.05 & 0.75 & 18.56 & 1.69 & 18.55 & 17.46 & 17.41 & $0.86,1.83$ & 1.89 \\
\hline $3.3,0.92$ & $\mathrm{H}^{d}$ & 一 & - & 一 & - & - & - & - & 17.22 & 17.26 & $1.15,1.90$ & - \\
\hline 0.48 & $\mathrm{~K}_{s}{ }^{d}$ & 一 & 一 & 一 & 一 & 一 & 一 & 一 & 16.94 & 17.07 & $0.81,1.67$ & 一 \\
\hline Mkn $178^{f}$ & $\mathrm{~J}$ & $20.62 \pm 0.11$ & $0.28 \pm 0.01$ & 12.94 & 0.39 & 14.36 & 0.62 & 13.41 & 12.78 & 12.70 & $0.34,0.57$ & 1.27 \\
\hline \multirow[t]{2}{*}{$\star$} & $\mathrm{H}^{d}$ & $19.87 \pm 0.18$ & $0.26 \pm 0.02$ & 12.35 & 0.26 & 14.85 & 0.51 & 12.92 & 12.29 & 12.25 & $0.34,0.58$ & 1.12 \\
\hline & $\mathrm{K}^{\prime d}$ & $19.92 \pm 0.43$ & $0.27 \pm 0.04$ & 12.31 & 0.36 & 14.00 & 0.52 & 12.91 & 12.13 & 12.01 & $0.36,0.58$ & 0.97 \\
\hline Mkn $1329^{e}$ & $\mathrm{~J}$ & $19.25 \pm 0.04$ & $0.67 \pm 0.01$ & 12.81 & 0.99 & 14.61 & 2.32 & 13.01 & 12.64 & 12.60 & $1.12,1.97$ & 1.01 \\
\hline $1.6,0.70$ & $\mathrm{H}^{d}$ & $18.81 \pm 0.01$ & $0.71 \pm 0.01$ & 12.25 & 1.01 & 13.85 & 2.09 & 12.57 & 12.00 & 11.94 & $1.17,2.08$ & 1.11 \\
\hline 0.68 & $\mathrm{~K}_{s}$ & $18.24 \pm 0.06$ & $0.60 \pm 0.01$ & 12.03 & 0.79 & 14.56 & 2.09 & 12.23 & 11.95 & 11.93 & $1.09,1.87$ & 0.72 \\
\hline IC $4662^{e}$ & $\mathrm{~J}$ & $16.79 \pm 0.24$ & $0.15 \pm 0.01$ & 10.60 & 0.55 & 10.32 & 0.83 & 10.73 & 9.68 & 9.64 & $0.29,0.53$ & 1.41 \\
\hline $4.8,0.97$ & $\mathrm{H}$ & $16.61 \pm 0.73$ & $0.15 \pm 0.02$ & 10.36 & 0.58 & 9.64 & 0.69 & 10.66 & 9.15 & 9.05 & $0.29,0.52$ & 1.37 \\
\hline 0.48 & $\mathrm{~K}_{s}{ }^{d}$ & 一 & - & 一 & 一 & - & - & - & 9.04 & 8.93 & $0.27,0.48$ & 1.75 \\
\hline $\mathrm{UM} 448^{e}$ & $\mathrm{~J}$ & $20.55 \pm 0.07$ & $3.83 \pm 0.10$ & 13.48 & 4.26 & 12.50 & 8.66 & 13.92 & 12.17 & 12.15 & $1.28,3.63$ & 2.33 \\
\hline \multirow[t]{2}{*}{$\star$} & $\mathrm{H}$ & $19.95 \pm 0.17$ & $4.02 \pm 0.31$ & 12.77 & 4.08 & 11.88 & 7.60 & 13.39 & 11.52 & 11.50 & $1.32,3.94$ & 2.40 \\
\hline & $\mathrm{K}_{s}$ & $19.55 \pm 0.15$ & $3.61 \pm 0.19$ & 12.61 & 4.01 & 11.53 & 8.16 & 13.05 & 11.22 & 11.30 & $1.21,3.24$ & 2.44 \\
\hline
\end{tabular}

$a$ : All values are corrected for Galactic extinction, adopting the $A_{B}$ from Tab. I.1 (cf. Sect. I.2.4.2)

$b, c$ : See Sect. I.3.2 for details. Objects whose LSB component has been modelled by a pure exponential fit (Eq. I.5) are marked with an asterisk.

$d$ : Decomposition uncertainty higher (faint object, or data affected by nearby bright stars).

$e$ : Calibration obtained at the ESO NTT, cf. Sect. I.2.4.

$f$ : Calibrated using field stars from the 2MASS catalogue, cf. Sect. I.2.4

$g$ : Fit radius interval and $(b, q)$ adopted from F01. 
beyond a transition radius $R_{\mathrm{T}}^{*}$ (cf. P96a, C02b), where optical and optical-NIR color gradients vanish and isophotes become more regular. In addition, whenever available, we used $\mathrm{H} \alpha$ maps to trace the size of the SF component, and get from it an additional constraint to $R_{\mathrm{T}}^{*}$. The LSB emission has been fitted out to the radius where SBPs became increasingly uncertain as a result of background noise (typically for $\$ 0.5 \sigma_{\mathrm{bgr}}$ for method iv). The fitted SBP range in $J$ is indicated with the dashed-gray line at the bottom of each profile (Figs. I.2-I.14).

An exponential fitting law

$$
I\left(R^{\star}\right)=I_{\mathrm{E}, 0} \exp \left(-\frac{R^{\star}}{\alpha}\right)
$$

with a central intensity $I_{\mathrm{E}, 0}$ and an exponential scale length $\alpha$ has been found to approximate well the intensity distribution of our sample galaxies in their LSB component. An exponential law is also known to fit well the outer parts of dwarf irregulars (dIs, Patterson \& Thuan 1996, van Zee 2000) and dwarf ellipticals (dEs, Vigroux et al. 1988, Binggeli \& Cameron 1991) over a few scale lengths.

However, for several of our sample BCDs, an extrapolation of the exponential LSB slope to smaller radii yields for $R^{*} \sim 1 \ldots 3 \alpha$ a higher intensity than the observed value. Thus, a meaningful decomposition of such SBPs cannot be achieved on the usual assumption that the exponential law is valid in the LSB component all the way to $R^{*}=0^{\prime \prime}$. Instead, one has to adopt an alternative fitting formula, approaching the exponential law for large radii and flattening in its inner part. Such a distribution should be compatible to the "type II" profiles of disc galaxies (Freeman 1970, see also MacArthur et al. 2002), or the "type V" profiles described for spheroidal early type DGs by Binggeli \& Cameron (1991).

Previous optical studies have allowed for the detection and modelling of such an inwards flattening exponential LSB profile in a few BCDs only (e.g. I Zw 115, P96a; Tol 65, Papaderos et al. 1999, hereafter P99; Tol 1214-277, Fricke et al. 2001, hereafter F01; SBS 0940+544, Guseva et al. 2001). However, SBPs of this kind do not appear to be rare among intrinsically faint dEs and dIs. They have been observed in several dEs with and without a central nucleus (cf. Binggeli \& Cameron 1991, Cellone et al. 1994, Young \& Currie 1994, 1995), and Vennik et al. (2000) deduce a fraction of $>10 \%$ for the SBPs of late-type dwarf galaxies falling into this category. In fact, a casual inspection of the sample of Patterson \& Thuan (1996), Makarova et al. (1998), Vennik et al. (2000) and van Zee (2000) reveals several examples of dIs showing an exponential outer LSB slope and a pronounced flattening for intermediate to small radii (e.g., UGC 2034, UGC 2053, UGC 5423, UGC 9128, UGC 10669 in the sample of Patterson \& Thuan 1996, KKH35 and KKSG 19 in Makarova et al. 1998, or UGCA 9, UGCA 15, UGC 2345,UGC 9240, UGC 10445 in the sample by van Zee 2000 ). Other examples are the dIs Holmberg I (Ott et al. 2001), Holmberg II (Noeske 1999), H 1032 2722 (Duc et al. 1999) and Kar 50 (Davidge 2002). Following the nomenclature of Binggeli \& Cameron (1991) we shall henceforth refer to this type of SBPs as to type V.

The physical origin and exact form of type $\mathrm{V}$ profiles in puffed-up stellar systems has not yet been studied in detail, neither observationally nor theoretically. Therefore 
it is difficult to say which fitting formula approximates best their intensity distribution (see discussion in Sect. I.5.3). An empirical fitting function that yields by deprojection a finite luminosity density for $R^{*}=0^{\prime \prime}$ has been proposed in P96a, as

$$
I\left(R^{\star}\right)=I_{\mathrm{E}, 0} \exp \left(-\frac{R^{\star}}{\alpha}\right)\left\{1-q \exp \left(-P_{3}\left(R^{\star}\right)\right)\right\}
$$

with

$$
P_{3}\left(R^{\star}\right)=\left(\frac{R^{\star}}{b \alpha}\right)^{3}+\left(\frac{R^{\star}}{\alpha} \cdot \frac{1-q}{q}\right) .
$$

The modified exponential distribution Eq. (I.6), in the following referred to as med, flattens with respect to a pure exponential law inside of a cutoff radius $b \alpha$, and attains at $R^{*}=0^{\prime \prime}$ an intensity given by the relative depression parameter $q=\Delta I / I_{\mathrm{E}, 0}<1$. An advantage of the med is that its exponential part (left part of Eq. I.6), depending on $I_{\mathrm{E}, 0}$ and $\alpha$ only, can be readily constrained by fitting Eq. I.5 to the outer exponential part of a type $\mathrm{V}$ profile. This has been done after SBPs were splined to equidistant radius steps, in order to ensure that the fit solution is not biased through a clustering of points to a specific radius range. Once the scale length $\alpha$ is fixed, the multiplicative right-hand side part of Eq. (I.6) is a function of $b$ and $q$, only. Because the $(b, q)$ parameter space has not been explored so far, e.g., by fitting Eq. (I.6) to a sample of dEs with type V SBPs, it is difficult to judge which values are appropriate for dwarf galaxies falling into this category. P96a inferred a tentative ratio of $b / q \sim 3$, they noted, however, that this could be luminosity-dependent.

In order to deduce plausible constraints to $(b, q)$, we first subtracted from the $J$ image of each sample BCD most of the irregular starburst emission. The latter has been approximated by an unsharp-mask version of the original image (Sect. I.3.4), adjusted such that no regular emission from the LSB component was removed. H $\alpha$ exposures and color maps were used to further ascertain that the subtracted emission was not part of the underlying host galaxy. $J$ band exposures processed this way were then used to compute SBPs for the LSB component. These profiles, denoted $J_{\mathrm{LSB}}$, allow to better trace the LSB component down to smaller $R^{*}$ than the ones derived prior to partial subtraction of the starburst light. By fitting Eq. (I.6) to the $J_{\mathrm{LSB}}$ SBPs we derived $b, q$ (first column in Tab. I.2). In most cases, fit uncertainties are 0.1 and 0.05 for $b$ and $q$, respectively. No reliable $J_{\text {LSB }}$ profiles could be computed for Tol 1214-277. For this system we fixed $(b, q)$ to values inferred by F01 from optical VLT data.

Alternatively, type V $J_{\text {LSB }}$ profiles were fit with a Sérsic model (Sérsic 1968) of the form

$$
I\left(R^{\star}\right)=I_{\mathrm{S}, 0} \exp \left(-\frac{R^{\star}}{\beta}\right)^{1 / \eta} .
$$


The exponent ${ }^{6} \eta$ in Eq. (I.8) is a handy indicator of systematic deviations of the observed SBP from an exponential distribution. An $\eta<1$ corresponds to a convex profile, which might be compatible to a med distribution with $q>0$ (see discussion in Sect. I.5.3), whereas an $\eta>1$ indicates a concave profile which, in the special case of $\eta=4$, translates into the de Vaucouleurs law (see, e.g., Caon et al. 1993).

Note that Eq. (I.8) approximates well the projected light of a variety of stellar populations with both a nearly constant $\mathcal{M} / \mathcal{L}$ (ellipticals or bulges; Caon et al. 1993, Andredakis et al. 1995, Graham et al. 1996, early-type dwarfs; Cellone et al. 1994, Young \& Currie (1994) or a wide range in $\mathcal{M} / \mathcal{L}$ (for instance, $\mathrm{nE}$ BCDs or the plateau component in the SBP of iE BCDs; P96a). Therefore a Sérsic exponent $\eta>1$ gives no strong indication for a composite stellar system like a BCD being similar to an intrinsically luminous early-type galaxy (see also discussion in $\mathrm{C} 02 \mathrm{~b}$ ).

The Sérsic exponents of the LSB components $\left(\eta_{\mathrm{LSB}}\right)$ were obtained from nonweighted fits to the $J_{\mathrm{LSB}}$ SBPs (see above) of type V profiles. This limits $\eta_{\mathrm{LSB}}$ to values $<1$. We avoided to fit Eq. (I.8) to the outer part of SBPs alone, i.e. for $R^{*} \geq R_{\mathrm{T}}^{*}$, as solutions obtained this way are very uncertain and depend strongly on the accuracy of the sky subtraction (see Sect. I.5.3 and detailled discussion in C02b). Notwithstanding the fact that Eqs. (I.6) and (I.8) give comparably good fits in terms of $\chi^{2}$, we decided not to include the full $\left(I_{\mathrm{S}, 0}, \beta, \eta\right)$ Sérsic solutions for the $J_{\mathrm{LSB}}$ SBPs in Tab. I.2. Instead, in its Col. 1 , we quote only the Sérsic exponent $\eta_{\mathrm{LSB}}$, the uncertainties of which are estimated to be of the order of $20 \%$.

The photometric quantities of the sample BCDs are summarized in Tab. I.2. BCDs without signatures of a type $\mathrm{V}$ profile in their underlying LSB component are marked with an asterisk in column 1 . For the remaining systems we list the $(b, q)$ and $\eta_{\text {LSB }}$ parameters, as obtained respectively by fitting Eqs. (I.6) and (I.8) to $J_{\mathrm{LSB}}$ SBPs. Columns 3 and 4 list, respectively, the extrapolated central surface brightness $\mu_{\mathrm{E}, 0}\left(\mathrm{mag} / \square^{\prime \prime}\right)$ and exponential scale length $(\mathrm{kpc})$, obtained by fitting Eq. (I.5) to the outer exponential LSB part of each SBP. Column 5 lists the total apparent magnitude of the LSB component, computed by extrapolating the fitted model (i.e. Eq. I.5 or Eq. I.6) to $R^{\star}=\infty$. Columns 6 through 9 list the radii and magnitudes of the star-forming $(\mathrm{P})$ and underlying stellar LSB component (E), as obtained by profile decomposition. Following P96a, we measure the respective radial extent $\left(P_{\text {iso }}, E_{\text {iso }}\right)$ and encircled magnitude $\left(m_{P_{\text {iso }}}, m_{E_{\text {iso }}}\right.$ ) of each component at an isophotal level $i s o$, taken to be $23 \mathrm{mag} / \square^{\prime \prime}$ for $J$ and $22 \mathrm{mag} / \square^{\prime \prime}$ for $H$ and $K$. The isophotal radii determined for the sample BCDs at $23 \mathrm{~J} \mathrm{mag} / \square^{\prime \prime}$ turn out to be comparable to those obtained from optical SBPs at $25 B$ mag/ $\square^{\prime \prime}$ ( $P_{25}$ and $E_{25}$ in P96a). Column 10 lists the magnitude from an SBP integration out to the last data point, and total magnitudes from aperture measurements (cf. Sect. I.3.3) are listed in column 11. The radii $r_{\text {eff }}$ and $r_{80}$, enclosing 50\% and $80 \%$ of the SBP's flux are included in column 12. Finally, a formal Sérsic exponent for the whole $\operatorname{SBP}\left(\eta_{\mathrm{SBP}}\right)$, for later comparison with literature data, is listed in column 13 of Tab. I.2.

\footnotetext{
${ }^{6}$ For consistency with, e.g., Caon et al. (1993) and C02b we define here the Sérsic shape parameter as $\eta$, i.e. the reciprocal exponent in Eq. (I.8). Note that in other studies (Cellone et al. 1994, Young \& Currie 1994, 1995; P96a) the Sérsic shape parameter is referred to as $1 / \eta$.
} 


\section{I.3.3 Aperture photometry and total magnitudes}

Since a SBP can only be accurately derived in regions with a sufficiently high $S / N$ (i.e., in general, down to the minimum intensity level in which the morphology of a BCD can still be visually checked and the problem of source confusion can be handled; cf. Sect. I.3.1), profile integration out to the last measured data point may, in some cases, underestimate the object's total flux. For extended LSB sources, or when NIR SBPs do not go sufficiently deep (e.g., the $K_{s}$ SBP of Tol 1214-277), the fractional flux missed can easily exceed $10 \%$. Similar problems may affect growth curve flux determinations, since these basically rely on a crude SBP derivation and can sensitively depend on the quality of background subtraction.

We therefore measured total magnitudes within polygonal apertures which extend typically out to 1.5 Holmberg radii (col. 11 of Tab. I.2), after removal of fore- and background sources from the area of interest (cf. Sect. I.2.3). Errors take into account the Poisson noise and small-scale variations of the local background.

Magnitudes and colors of selected features, such as stellar clusters or H II regions, have been corrected for the flux contribution of the underlying LSB component, by interpolating the mean surface brightness of adjacent regions. Values computed this way are marked with the superscript $\ddagger$. As pointed out in Cairós et al. (2002a) and Papaderos et al. 2002, corrections for the LSB emission are generally not negligible in optical wavelengths. That this statement is also true in the NIR domain is illustrated on the example of the iE BCD UM 461 (Fig. I.1); correction for the flux contribution of the LSB background shifts even the brightest SF region (a, $m_{J} \ddagger=17.26 \mathrm{mag}$, open circle) of this system by $+0.25 \mathrm{mag}$ and $-0.1 \mathrm{mag}$ in the $J-H$ and $H-K s$ color diagram. A census and photometric study of compact stellar clusters in the extended BCD sample included in this project after correction for the LSB- and ionized gas emission will be presented in Cairós et al. (2003).

\section{I.3.4 Unsharp masking}

The considerable intensity range of a $\mathrm{BCD}$, from its faint LSB outskirts to the brightest nuclear starburst region, renders the detection of fine coherent morphological features in the central portion of the galaxy difficult. We use therefore a modified unsharp masking technique (cf., e.g., Papaderos 1998), referred to in the following as hierarchical binning $(\mathrm{hb})$ - transformation. This contrast-enhancing procedure is stable against noise at low intensities and allows for the flux determination of faint sources within the bright background of a BCD, with an angular size smaller than a user-defined value. Morphological features of interest revealed using this procedure are displayed in the grayscale/isophote insets of the sample galaxies, and described in Sect. I.4.

\section{I.3.5 Colors of the underlying LSB host galaxy}

Colors of the LSB host galaxy were derived as the error-weighted mean of the color profiles for $R^{*}>R_{\mathrm{T}}^{*}$, after rejection of deviant points, being probably affected by uncertainties in the sky determination, and local residuals in the subtraction of background 


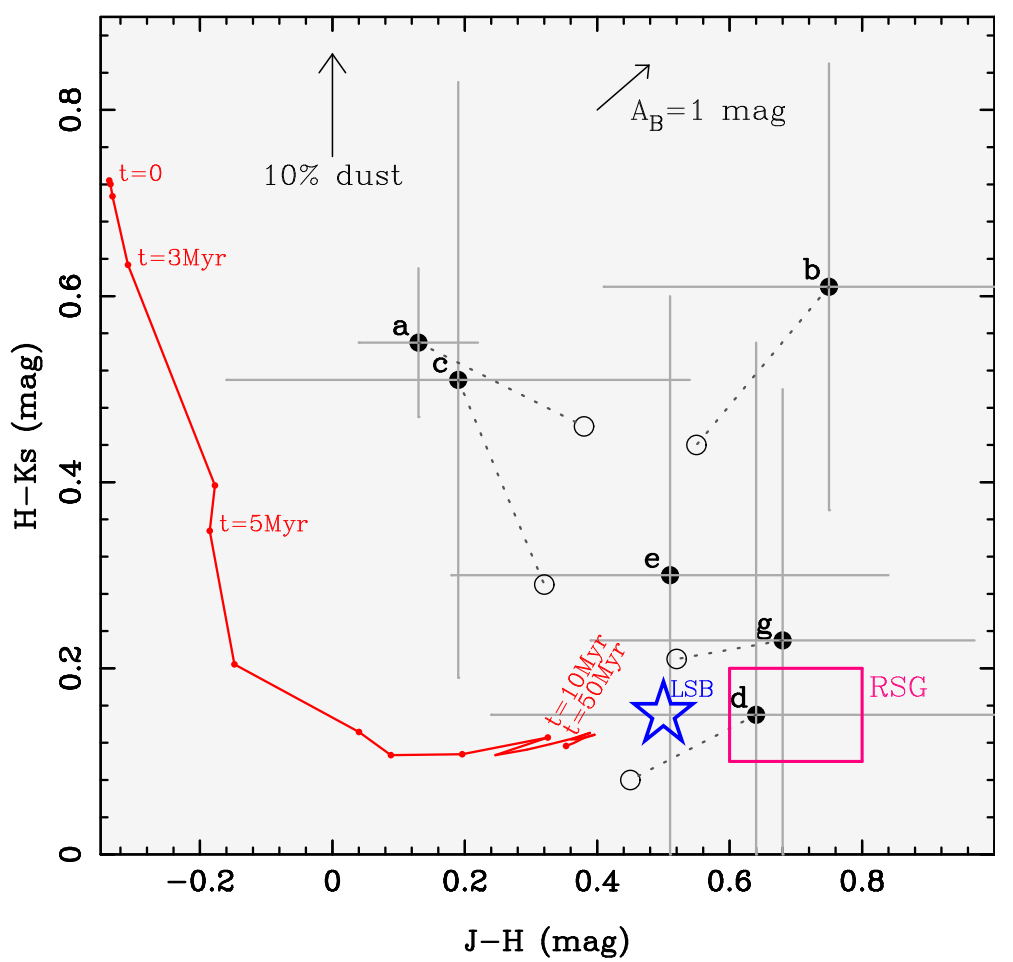

Fig. I.1. NIR two-color diagram for the brightest compact regions in the iI BCD UM 461 (from Cairós et al. 2003). Filled circles show the colors of the regions a through $g$ indicated in Fig. I.5, after correction for the flux contribution of the underlying LSB host galaxy. Open circles, connected with dotted lines, indicate the color of the respective region prior to that correction. The star marks the color of the stellar host galaxy ("LSB"). The color range covered by red supergiants in the SMC (Elias et al. 1985) is shown by the box labelled RSG to the lower right. The temporal evolution of the $J-H$ vs. $H-K_{s}$ color for an instantaneously formed stellar population with $Z_{\odot} / 10$ for an age between $t=0$ and $t=50 \mathrm{Myr}$ (solid line) has been calculated with the PEGASE code (Fioc \& Rocca-Volmerange 1997). Arrows depict the effect of a 10\% contribution of warm ( $\sim 200 \mathrm{~K}$ ) dust to the $K$ band (cf. Campbell \& Terlevich 1984), as well as the extinction vector. All colors are transformed to the 2MASS photometric system.

sources. For Tol 1400-411, Pox 4, Mkn 178 and IC 4662, uncertainties in the LSB colors are larger, due to extended starburst emission or crowding with nearby bright stars. As for the very metal-deficient systems Tol 65 and Tol 1214-277, the faintness of their LSB component in $K_{s}$ has not permitted, despite generous exposure times, to pin down their $H-K_{s}$ colors.

Whenever calibrated optical data were available, optical-NIR colors were derived. Because the quality of the SBPs was typically better in $J$ than in $H$, and the $J-H$ color shows little evolution with time (few $0.1 \mathrm{mag}$ ) for old ( $\gtrsim 1 \mathrm{Gyr}$ ) stellar populations, we derived $B-J$ colors instead of the more commonly used $B-H$ colors.

The mean colors of the host galaxies are shown at the right edge of each color profile (Figs. I.2 - I.14), in the photometric system in which the respective galaxy was observed and calibrated (see Sect. I.2.4.1). Table I.3 lists the NIR colors, transformed to the 2MASS photometric system to facilitate comparisons and the $B-J$ color, where 
available. Errors give cumulative uncertainties in the calibration, transformation to the 2MASS system, and the scatter and systematic uncertainty of each color profile (see also Sect. I.5.4).

Table I.3. Colors of the host galaxy ${ }^{a}$

\begin{tabular}{llll}
\hline Object & $\begin{array}{l}J-H \\
{[\mathrm{mag}]}\end{array}$ & $\begin{array}{l}H-K_{s} \\
{[\mathrm{mag}]}\end{array}$ & $\begin{array}{l}B-J \\
{[\mathrm{mag}]}\end{array}$ \\
\hline Tol3 & $0.55 \pm 0.07$ & $0.10 \pm 0.06$ & - \\
Tol 65 $^{c}$ & $0.48 \pm 0.17^{b}$ & - & $1.3 \pm 0.15^{b}$ \\
Tol 1214-277 & $0.39 \pm 0.16^{b}$ & - & $1.0 \pm 0.13^{b}$ \\
Tol 1400-411 $^{c}$ & $0.43 \pm 0.08$ & $0.26 \pm 0.09$ & $1.45 \pm 0.16$ \\
Pox 4 & $0.42 \pm 0.13$ & $0.12 \pm 0.13$ & - \\
Pox 4B & $0.43 \pm 0.15^{b}$ & $0.1 \pm 0.15^{b}$ & - \\
UM 448 & $0.69 \pm 0.10^{b}$ & $0.14 \pm 0.11^{b}$ & $2.09 \pm 0.15$ \\
UM 461 & $0.50 \pm 0.06$ & $0.15 \pm 0.06$ & $1.92 \pm 0.16$ \\
He 2-10 & $0.59 \pm 0.06$ & $0.11 \pm 0.07$ & - \\
IC 4662 & $0.57 \pm 0.12^{b}$ & $0.03 \pm 0.12^{b}$ & - \\
Mkn 178 & $0.53 \pm 0.15$ & $0.25 \pm 0.16^{b}$ & $2.00 \pm 0.15$ \\
Mkn 1329 & $0.65 \pm 0.09$ & $0.03 \pm 0.10$ & - \\
Haro 14 & $0.69 \pm 0.12^{b}$ & $0.20 \pm 0.12^{b}$ & $2.02 \pm 0.15$ \\
\hline
\end{tabular}

${ }^{a}$ : Corrected for galactic extinction (see Sect. I.2.4.2); NIR colors are transformed to the 2MASS system (cf. Sect. I.2.4.1). The $B$ band denotes the Johnson $B$. Errors include cumulative uncertainties in the determination of color profiles, the calibration and the transformation to the 2MASS system.

${ }^{b}$ : possible local instabilities in one SBP at low $S / N$ levels.

$c$ : possible contamination by gas emission over a large portion of the LSB host galaxy

\section{I.4 Results and discussion of individual objects}

\section{I.4.1 Tol 3 (NGC 3125)}

This luminous $\left(M_{B} \sim-18.0\right.$; Marlowe et al. 1999) and relatively metal-rich $\left(Z_{\odot} / 6 \ldots\right.$ $Z_{\odot} / 3$; Kobulnicky et al. 1999, Schaerer et al. 1999, Marlowe et al. 1999 and references therein) BCD is known to be a member of the NGC 3175 galaxy group (García 1993). NIR and optical images reveal two compact ( $\$ 0.3 \mathrm{kpc}$ ) high-surface brightness (HSB) regions: the brighter northwestern knot $\mathrm{A}$, roughly coinciding with the geometrical center of the smooth LSB host galaxy, and the fainter knot B, located $\sim 1 \mathrm{kpc}$ southeast of A (see Fig. I.2a). Either knot is the locus of ongoing star formation, as witnessed by the detection of Wolf-Rayet features (Kunth \& Sargent 1981, Vacca \& Conti 1992, Schaerer et al. 1999) and red supergiants from CO absorption studies (Campbell \& Terlevich 1984). The intense SF activity in Tol 3 is also reflected on relatively blue optical colors ( $B-V=0.24, V-I=0.28$; Marlowe et al. 1997) in its nuclear region, as 

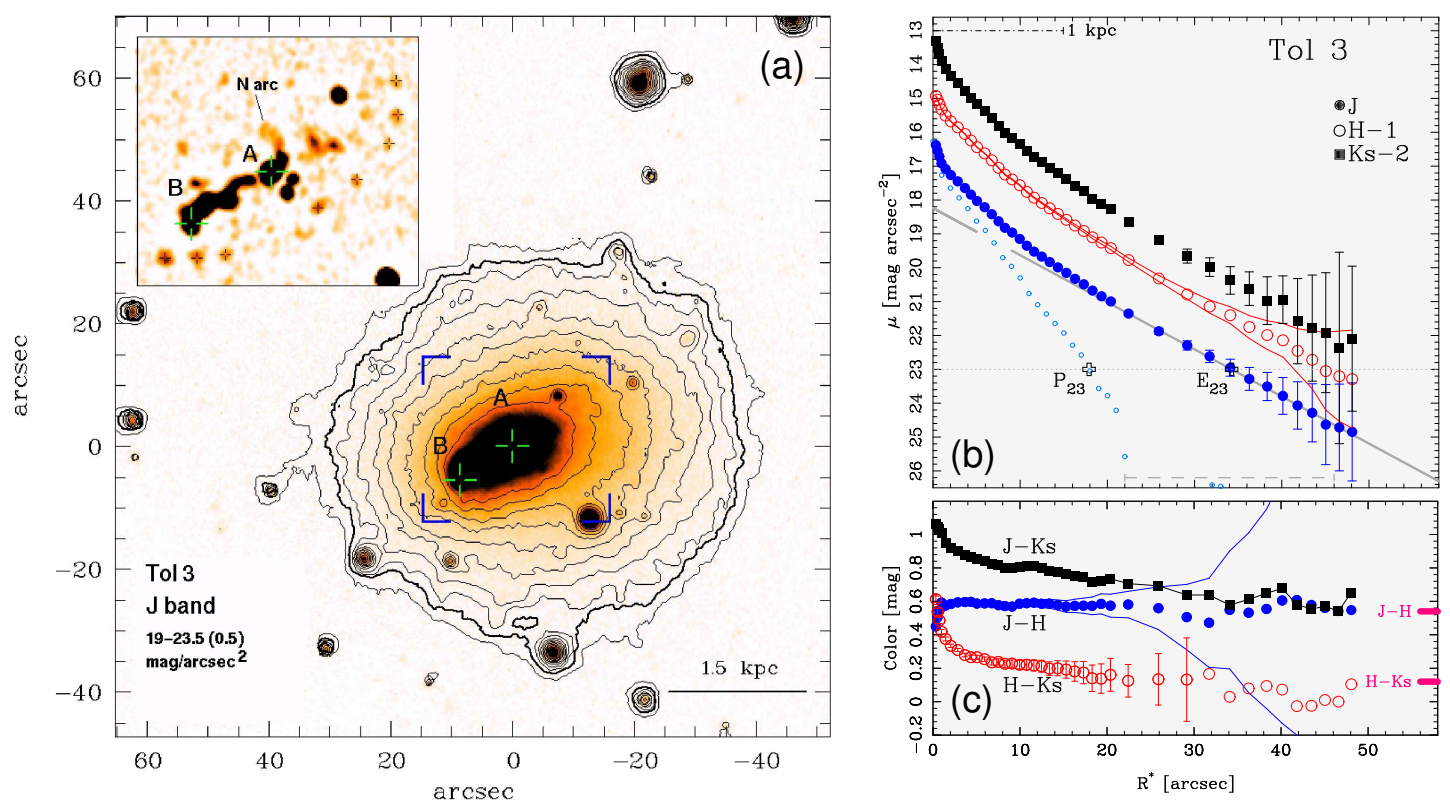

Fig. I.2. a): Contours overlaid with a $J$ image of Tol $3(D=13.8 \mathrm{Mpc})$. North is up, east to the left. Contours, corrected for Galactic extinction, go from 19 to $23.5 \mathrm{~J}$ mag/ $\square^{\prime \prime}$ in increments of $0.5 \mathrm{mag}$. The $23 J \mathrm{mag} / \square^{\prime \prime}$ isophote is illustrated by the thick contour. The brighter star-forming region A and the fainter knot B (following the denomination by Schaerer et al. 1999) are marked with crosses. The inset shows a contrast-enhanced (see Sect. I.3.4) and magnified version of the central region of the BCD (indicated by brackets in the contour map image). Compact sources arranged along the southwestern arc-like chain are marked with small crosses. b): Surface brightness profiles (SBPs) of Tol 3 in the $J, H$ and $K_{s}$, corrected for galactic extinction. For a better visualization, the $H$ and $K_{s}$ SBPs are shifted by -1 and -2 mag, respectively. The thick solid line illustrates an exponential fit to the stellar LSB component in $J$ (cf. Sect. I.3.2), computed in the radius range indicated by the light gray, long-dashed bar at the bottom of the figure. The emission in excess to the fit (small open circles) is attributable to the starburst component, which dominates the light in the inner part of Tol 3. The isophotal radii $P_{23}$ and $E_{23}$ of the star-forming and LSB component at the surface brightness level of $23 \mathrm{~J}$ mag/ $\square^{\prime \prime}$ (horizontal dotted line) are indicated. The bar at the upper left of the figure corresponds to a galactocentric distance of $1 \mathrm{kpc}$. (c): Color profiles, computed by subtraction of the SBPs shown in the upper right panel. The thick lines at the rightmost part of the diagram indicate the mean $J-H$ and $H-K_{s}$ colors of the LSB component (see Sect. I.3.5).

well as on copious $\mathrm{H} \alpha$ emission $\left(1.1 \times 10^{41} \mathrm{erg} \mathrm{s}^{-1}\right.$; Marlowe et al. 1997). Deep H $\alpha$ imaging by Marlowe et al. (1995) revealed a bipolar outflow roughly perpendicular to the major axis of the $\mathrm{BCD}$, extending out to $\sim 2.8 \mathrm{kpc}$ from its nuclear region.

Unsharp-masked NIR images reveal a complex morphology in the HSB regime of the BCD (inset in Fig. I.2a). Regions A and B are immersed in an extended "S-shaped" pattern, $\sim 0.9 \mathrm{kpc}$ in length, ending at its NW tip with a curved feature ( $\mathrm{N}$ arc). There is some evidence for propagation of SF activities, as both archival optical NTT and NIR data reveal signatures of a younger age and stronger ionized gas emission towards region B (cf. Fig. I.3, bottom). For region A we determine within a rectangular $4^{\prime \prime} \times 4^{\prime \prime}$ 

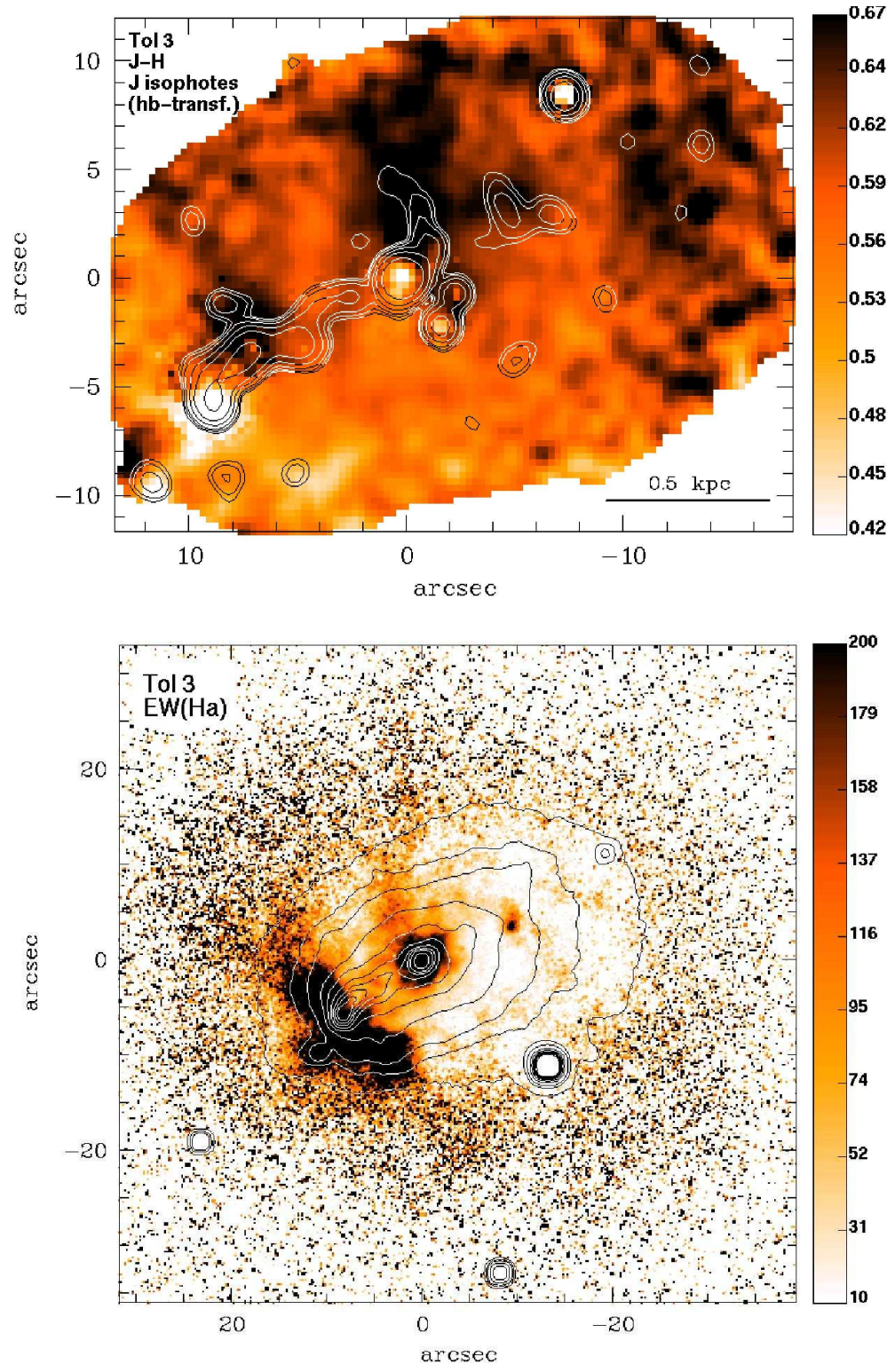

Fig. I.3. top: $J-H$ color map of the central region of Tol 3, corrected for Galactic extinction. The overlaid $J$ contours are computed from the contrast-enhanced blow-up included in Fig. I. 2 (see text for details). bottom: $\mathrm{EW}(\mathrm{H} \alpha)$ map of Tol 3, overlaid with $R$ contours.

aperture colors of $J-H=0.61\left(J-H^{\ddagger}=0.56\right)$ and $H-K=0.41\left(H-K^{\ddagger}=0.58\right)$. For knot B we infer a $J-H=0.39\left(J-H^{\ddagger}=0.14\right)$ and $H-K=0.48\left(H-K^{\ddagger}=0.71\right)$. Such colors suggest a younger stellar age together with an appreciable ionized gas contribution towards the SE part of the SF component. The latter is verified from the $\mathrm{H} \alpha$ equivalent width (EW) map in Fig. I.3 (bottom) (see also Gil de Paz et al. 2002) which shows that $\mathrm{SE}$ of knot $\mathrm{B}$, and all over an extended rim perpendicular to the major axis of the $\mathrm{BCD}$, the $\mathrm{EW}(\mathrm{H} \alpha)$ rises to $>200 \AA$.

Interestingly, unsharp-masked NIR and optical images reveal on larger scales a chain of faint knots (depicted with crosses in the inset of Fig. I.2a), arranged over $\sim 2.5$ 
kpc SW of regions A and B. Their typical $J^{\ddagger}$ magnitudes of $\sim 20.6 \ldots 19$ mag translate into absolute magnitudes of $-10 \ldots-12 \mathrm{mag}$. The nature and formation history of this extended feature is intriguing. One interpretation is that it delineates the approaching side of an oblate star-forming shell triggered by the burst, or that it may be associated with an inclined circumnuclear disk of $\sim 1 \mathrm{kpc}$ in radius. The first hypothesis is consistent with the findings by Alton et al. (1994), who suggested from imaging-polarimetry the presence of a large-scale bipolar reflection dust-nebula illuminated by the central starburst region. This could provide effective UV-shielding, thereby allowing for secondary SF activity. The systematically redder colors in the NE half of Tol 3 (Fig. I.3, top) are also in line with the same hypothesis, if they originate from the receding, more strongly absorbed side of the galaxy.

SBPs in the NIR (Fig. I.2b) show in the radius range $22^{\prime \prime} \leq R^{*} \leq 48^{\prime \prime}$ an exponential intensity fall-off with a scale length $\alpha=0.52 \mathrm{kpc}$. This value is close to the $B$ scale length of $\alpha=0.48 \mathrm{kpc}$, inferred for the LSB component by Marlowe et al. (1997) within $\lesssim 30^{\prime \prime}$. By contrast, our surface photometry does not appear to be compatible to that of Kunth et al. (1988). These authors show optical SBPs out to a radius $R^{*}=80^{\prime \prime}$, by a factor of 1.6 larger than the study here or in Doublier et al. (1999) and up to 2.7 times larger than in Marlowe et al. (1997). NIR SBPs show no evidence for a dominant $R^{1 / 4}$ profile in Tol 3 (cf. Kunth et al. 1988, Doublier et al. 1999). A de Vaucouleurs profile is neither supported by the Sersic index $\eta=1.4$ we derive for the entire $J$ profile.

The NIR color profiles (Fig. I.2c) show minor gradients $\left(<0.15 \mathrm{mag} \mathrm{kpc}^{-1}\right)$ and level off to $J-H=0.54 \mathrm{mag}$ and $H-K=0.12 \mathrm{mag}$ for $R^{*}>20^{\prime \prime}$. These results are not compatible to Doublier et al. (2001), who report for $R^{*}>10^{\prime \prime}$ a roughly linear $J-H$ color increase from $\sim 0.5 \mathrm{mag}$ to $\sim 2.0 \mathrm{mag}$. A good agreement is found with Vanzi et al. (2002), who derive for the LSB component of Tol 3 colors of $J-H=0.6$ mag and $H-K=0.25 \mathrm{mag}$.

\section{I.4.2 Haro 14 (NGC 244)}

This relatively metal-rich $\mathrm{nE} \mathrm{BCD}\left(\mathrm{Z} \approx Z_{\odot} / 3\right.$, Hunter \& Hoffman 1999$)$ shows SF activity ontop a smooth, nearly circular stellar LSB host galaxy (Fig. I.4a). The intensity distribution of the latter (Fig. I.4b) is approximated best by a modified exponential distribution (Eq. I.6), flattening for $R^{*} \lesssim 20^{\prime \prime}$ (see the detailed discussion in Sect. I.5.2).

The central part of the BCD contains a massive complex of SF regions, displaced by $\sim 0.5 \mathrm{kpc}$ SW of the geometrical center of the outer LSB isophotes. This SF component measures $\approx 1.4 \mathrm{kpc}$ in diameter in the $J$ band (this paper) and $\sim 2 \mathrm{kpc}$ in the $\mathrm{H} \alpha$ line (Marlowe et al. 1997). Its composition out of several individual regions, reported by Doublier et al. (1999) from optical images, is also evident from the contrast-enhanced NIR images (Fig. I.4a, upper-left inset), which reveal a wealth of individual regions, spanning a range of $\gtrsim 3$ mag. The upper-right inset of Fig. I.4a reveals a chain of faint knots with a length of $\approx 1.5 \mathrm{kpc}$ (labeled "E loop"), extending eastwards from the main SF complex. There is a hint for a similar feature in the southwestern direction. Published data (e.g., the $\mathrm{H} \alpha$ image by Marlowe et al. 1997) do not allow to assess whether these faint features may trace induced star formation along supergiant shells, as might be hypothesized from their morphology. 

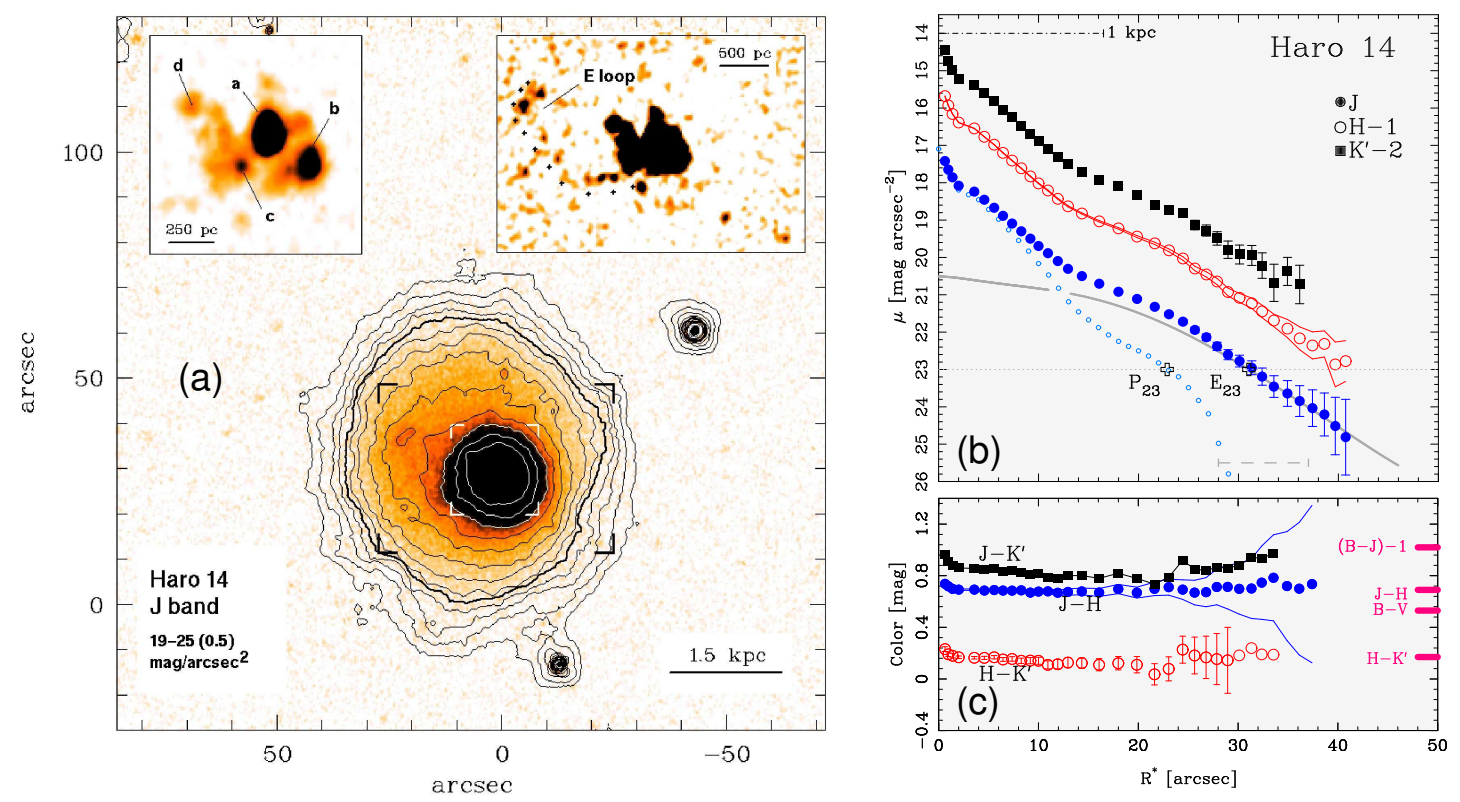

Fig. I.4. Haro 14 ( $D=12.5 \mathrm{Mpc})$. For explanations of symbols and labels, refer to Fig. I.2. a): $J$ band image and isophotes. Note that the center of the star-forming regions is offset by $\sim 0.5 \mathrm{kpc}$ from the center of the outer circular isophotes. The upper-left and upper-right insets show unsharp-masked versions of the central part of the BCD, marked in the main image by the white and black brackets, respectively. In the upper-left inset we mark regions a through $d$. The upper-right inset reveals an extended, curved chain of knots traceable up to $\sim 1.5 \mathrm{kpc}$ from the brightest region a. b),c): Surface brightness and color profiles. The thick grey line shows a fit to the LSB component using a modified exponential distribution Eq. (I.6) with $b, q=3.6,0.94$

For the two brightest regions, denoted $\mathrm{a}$ and $\mathrm{b}$, we obtain respective absolute magnitudes of $M_{\mathrm{J}}^{\ddagger}=-14.7 \mathrm{mag}$ and $-13.3 \mathrm{mag}$, and effective radii $<80 \mathrm{pc}$. If the mean $E(B-V)=0.35$ for Haro 14 (Hunter \& Hoffman 1999) applies to knots $\mathrm{a}$ and $\mathrm{b}$, then their de-reddened colors would be $J-H^{\ddagger} \sim 0.6$ and $H-K_{s}^{\ddagger} \sim 0.2$. Such colors are reached at the earliest when the NIR emission becomes dominated by red supergiants ( $\sim 10 \ldots 30 \mathrm{Myr}$ ), and suggest no strong nebular line contamination. This points against substantial ongoing star formation in regions $a$ and $b$.

The large spatial extent of SF sources in the inner portion of the BCD is further evidenced by the profile decomposition (Fig. I.4b), yielding a plateau radius $P_{23} \approx 1.4$ $\mathrm{kpc}$ in the $J$ band. The optical and NIR colors of the LSB host galaxy indicate an age of several Gyr, in agreement with previous estimates by Marlowe et al. (1999).

\section{I.4.3 UM 461}

Telles \& Terlevich (1995) suggested that the iI BCD UM 461 forms together with UM 463, UM 465 and UM 462 a loose group of dwarf galaxies.

SF activity is confined to the whole northeastern part of the galaxy, i.e. within the $21.5 \mathrm{~J} \mathrm{mag} / \square^{\prime \prime}$ isophote, or on a spatial scale of $\sim 0.9 \times 0.7 \mathrm{kpc}$. The two brightest SF 

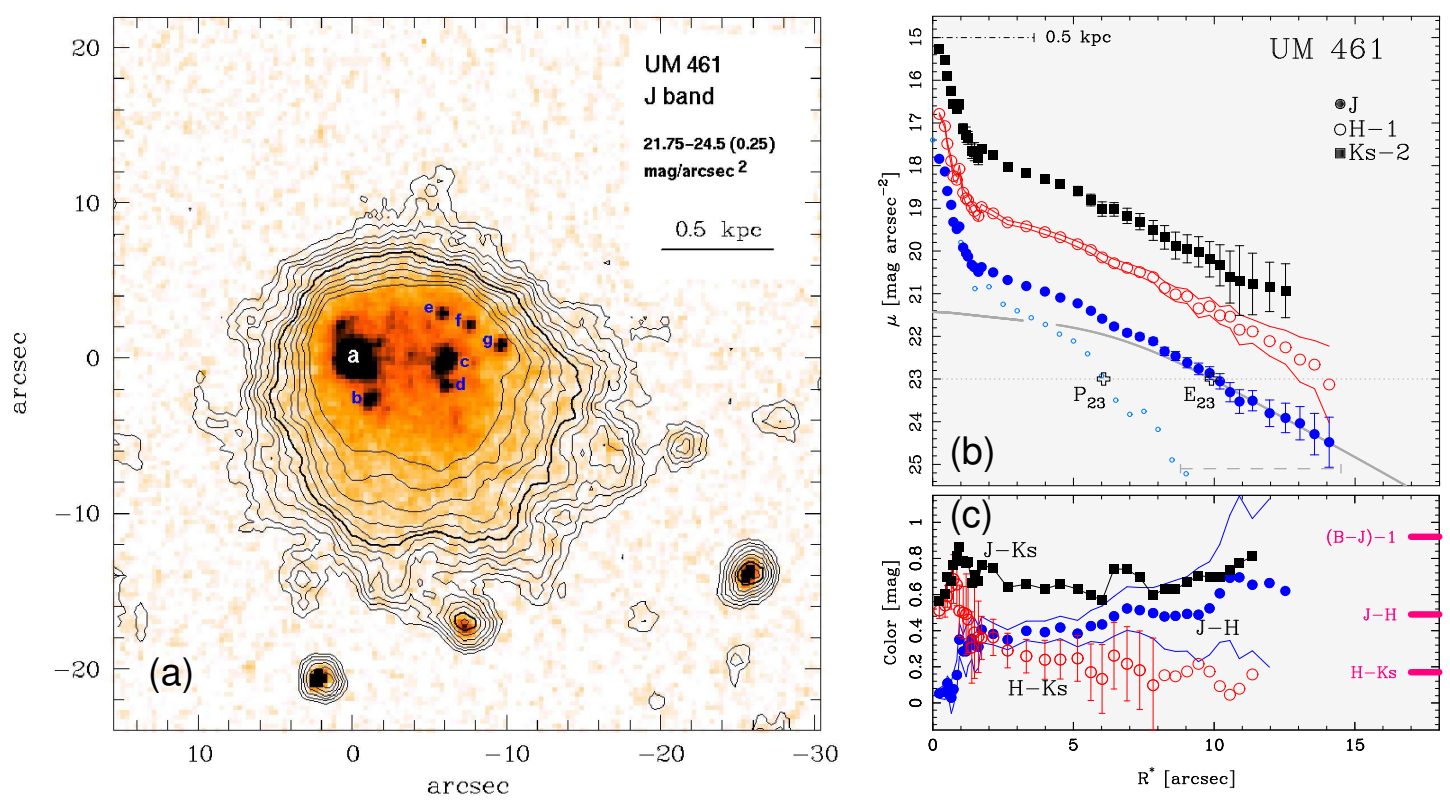

Fig. I.5. UM $461(D=14.3 \mathrm{Mpc})$. For explanations of symbols and labels, refer to Fig. I.2. a): $J$ band image and isophotes. Bright stellar assemblies in the central portion of the BCD are indicated. b),c): Surface brightness and color profiles. The thick grey line shows a fit to the LSB host galaxy using Eq. (I.6) with parameters $b, q=2.3,0.85$.

regions a $\left(\mathrm{m}_{J^{\ddagger}}^{\ddagger}=17.3 \mathrm{mag}\right.$; Fig. I.5a) and $\mathrm{c}\left(\mathrm{m}_{J^{\ddagger}}^{\ddagger}=19.5 \mathrm{mag}\right)$ in UM 461 are separated by $20 \mathrm{~km} \mathrm{~s}^{-1}$ in the velocity space. This difference is of the order of the intrinsic $\mathrm{H} \mathrm{I}$ velocity dispersion within UM $461\left(\sim 30 \mathrm{~km} \mathrm{~s}^{-1}\right.$; van Zee et al. 1998). The available data allow us to resolve a manifold of morphological features within the SF component, most notably a chain of compact sources northwest of region $c$ (labelled e-g) with a typical $M_{J} \ddagger-10.4$ mag. Their $J-H^{\ddagger}$ colors of 0.5-0.6 mag are consistent with the interpretation by Méndez \& Esteban (2000) that they are extinguished SF regions, formed not earlier than $100 \mathrm{Myr}$ ago. From profile decomposition we infer the summed up luminosity fraction of compact and diffuse SF sources to $\sim 37 \%$ of the $J$ band light of UM 461.

UM 461 shows in its faint outskirts a slight asymmetry towards the SW direction. The intensity profile of the LSB host can be approximated by a med model with a scale length $\alpha=0.21 \mathrm{kpc}$ and a depression parameter $q \approx 0.85$ (Fig. I.5b). The $J$ band scale length derived here is in excellent agreement with the value derived in the optical by Telles et al. (1997). Color profiles reflect the ongoing SF for small radii $\left(R^{*} \lesssim 2^{\prime \prime}\right)$, and approach mean values of $J-H=0.49 \mathrm{mag}$ and $H-K_{s}=0.17 \mathrm{mag}$ in the LSB periphery. Such colors, together with the $B-J \approx 1.9$ mag determined from optical data, point consistently to a relatively evolved stellar LSB background. The NIR colors derived here do not appear to be compatible with those by Doublier et al. (2001). These authors find within the radius range $5^{\prime \prime} \lesssim R^{*} \lesssim 10^{\prime \prime}$ the $J-H$ color to increase from 1.5 to $\sim 2.3 \mathrm{mag}$, whereas the $H-K$ color shows a continuous decrease from -0.5 mag to $<-1 \mathrm{mag}$. Also, the integrated $J-H$ and $H-K$ colors of $0.99 \mathrm{mag}$ and $-0.68 \mathrm{mag}$, 
respectively, listed in Doublier et al., differ significantly from the values of 0.47 mag and 0.2 mag derived in the present study.

\section{I.4.4 Henize 2-10 (ESO 495-G21)}
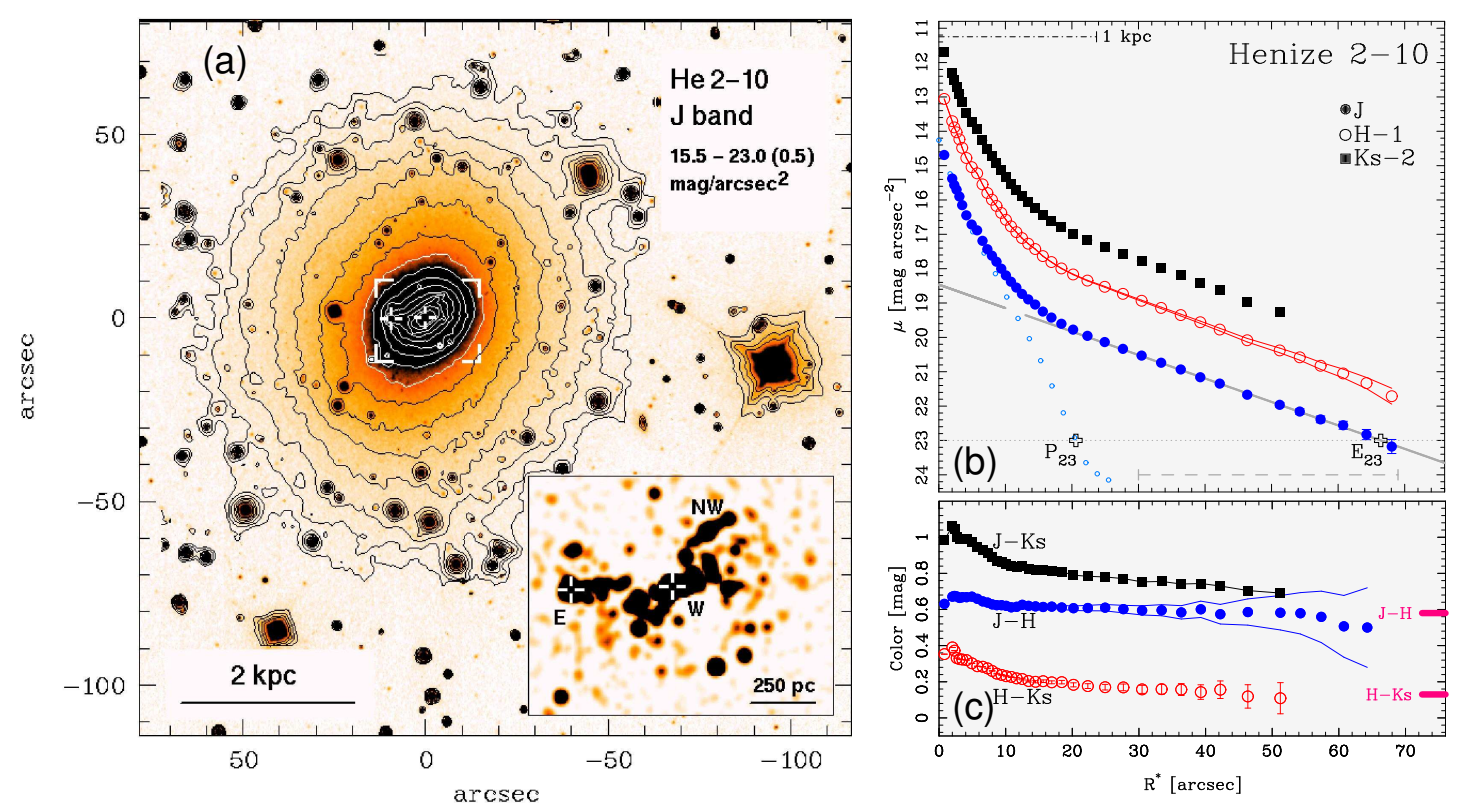

Fig. I.6. Henize $2-10(D=8.7 \mathrm{Mpc})$. For explanations of symbols and labels, refer to Fig. I.2. a): $J$ band image and isophotes. The inset shows an unsharp-masked version of the nuclear region of the BCD (region marked by the brackets in the main image). b),c): Surface brightness and color profiles.

This relatively metal-rich $\mathrm{iE} \mathrm{BCD}\left(Z_{\odot} / 2.4 \ldots z Z_{\odot}\right.$; Schaerer et al. 1999 , Kobulnicky et al. 1999) is the first extragalactic system in which the broad He II $\lambda 4686$ line was detected. The starburst nature of He 2-10 is evidenced by a chain of bright SuperStar Clusters (SSCs) in its brightest western SF region W (Conti \& Vacca 1994), extended X-ray and $\mathrm{H} \alpha$ emission (Hensler et al. 1997, Papaderos \& Fricke 1998), as well as a large bipolar outflow from the SF region (Papaderos \& Fricke 1998), with an expansion velocity between $\sim 250$ and $\gtrsim 360 \mathrm{~km} \mathrm{~s}^{-1}$ (Méndez \& Esteban 1999, Johnson et al. 2000).

Unsharp masking (inset in Fig. I.6a) reveals a wealth of morphological features in the nuclear region of He 2-10, most notably an extended feature protruding northwest of region W (labelled NW) and an arc-like chain of compact sources connecting the tip of region NW with the secondary SF knot E. Ground-based $B-R$ maps by Papaderos \& Fricke (1998) indicate that region NW and the concatenation of sources bending southwards of it are considerably bluer than the underlying LSB host galaxy.

Our SBPs (Fig. I.6b) show an exponential intensity decrease in the LSB component $\left(R^{*} \gtrsim 20^{\prime \prime}\right)$, with a $J$ scale length identical to that obtained previously from optical data ( $\alpha=0.67 \mathrm{kpc}$, Papaderos \& Fricke 1998). 


\section{I.4.5 Tol 1400-411 (NGC 5408)}
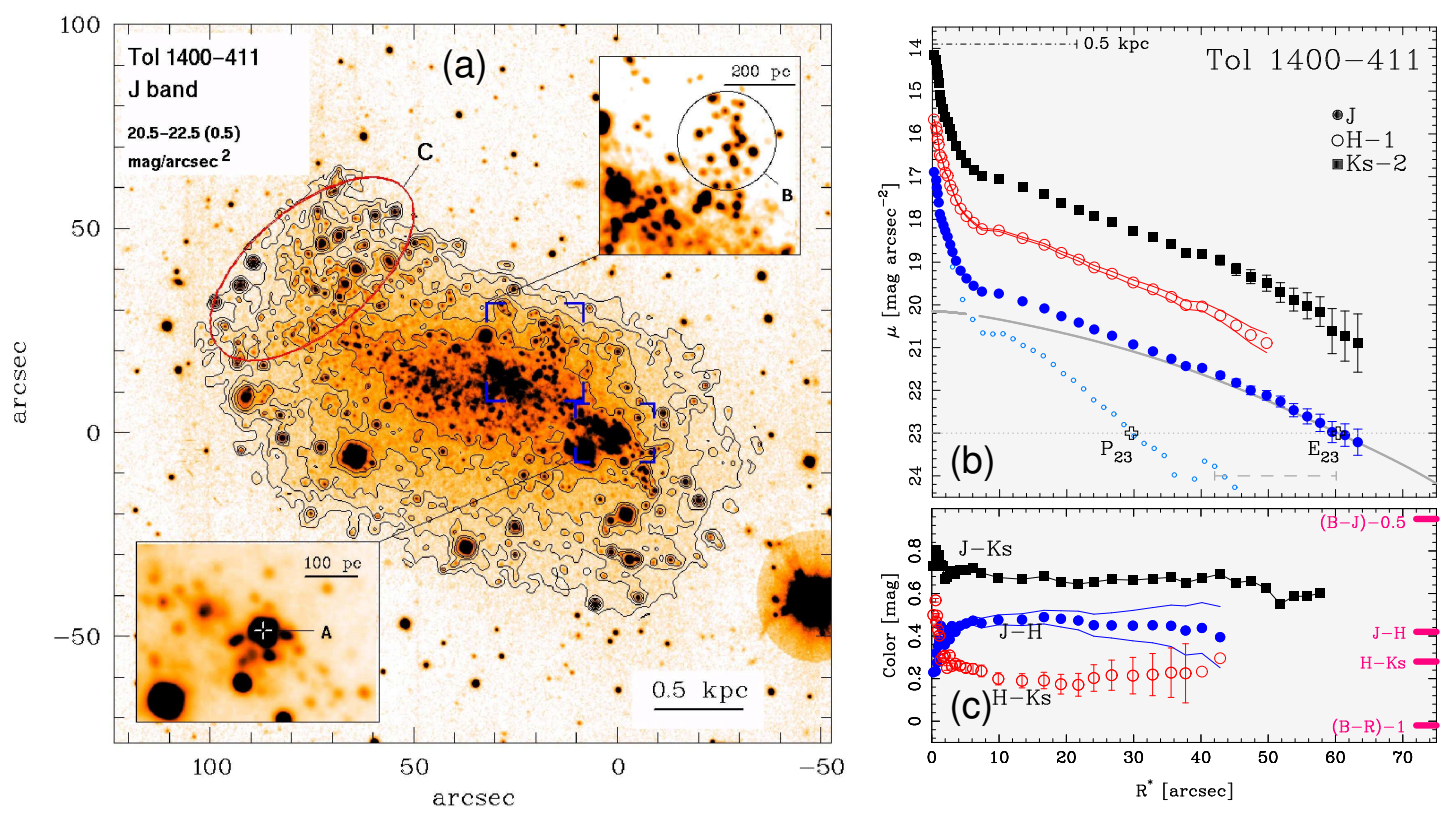

Fig. I.7. Tol 1400-411 ( $D=4.8 \mathrm{Mpc}$ ). For explanations of symbols and labels, refer to Fig. I.2. a): $J$ band image and isophotes. The insets show magnifications of the bright SW star-forming knot $A$ as well as the region $B$ extending northwestwards from the major axis. The bluer NE region $\mathrm{C}$ is marked. b),c): Surface brightness and color profiles. The thick grey line shows a fit to the host galaxy using Eq. (I.6) with $b, q=3.0,0.82$.

This cometary iI BCD is a member of the Cen A group (van den Bergh 2000), situated at a projected distance of $7.2^{\circ}(\sim 0.6 \mathrm{Mpc})$ from Cen A. Intense SF activity is taking place mainly at the SW part of its elongated stellar LSB host, where groundbased NIR and archival HST/WFPC2 optical data (PI: P. Seitzer, G0-8601) reveal three bright $\left(\mathrm{m}_{V} \lesssim 16.2\right)$ stellar clusters spread over $8^{\prime \prime}(\sim 200 \mathrm{pc})$. The appreciable ionized gas contribution in the SF component of Tol 1400-411 is reflected on the very blue $V-I$ color of $\lesssim-0.4 \mathrm{mag}$, in the brightest cluster, A (cf. 1.h.s. blow-up of Fig. I.7a) and the large $\operatorname{EW}(\mathrm{H} \beta) \gtrsim 250 \AA$ determined for this system by Masegosa et al. (1994).

Interferometric $21 \mathrm{~cm}$ line studies (Fritz 2000) show that the BCD is immersed within a large, rotationally supported $\mathrm{H}$ I cloud of about $8 \times 16 \mathrm{kpc}$ in size. The $\mathrm{H} \mathrm{I}$ halo reveals two surface density maxima, one coinciding with region $A$ and the other one located $\sim 1^{\prime}$ to the east.

The present data, as well as $\mathrm{H} \alpha$ images by Fritz (2000), reveal signatures of lowlevel SF activity all over the $22 J \mathrm{mag} / \square^{\prime \prime}$ isophote of the BCD, i.e $2.6 \mathrm{kpc}$ across. Interestingly, $30^{\prime \prime} \mathrm{NE}$ of region $\mathrm{A}$ our data show a nearly circular region, $\approx 12^{\prime \prime}$ in diameter, with relatively blue ( $B-R$ of $\lesssim 0.75, V-I \lesssim 0.45 \mathrm{mag}$ ) colors in its unresolved interior (denoted $B$ in Fig. I.7a). Such colors, being significantly bluer than those in the LSB host $(B-R \approx 1, V-I=0.6-0.7)$, suggest that region $\mathrm{B}$ is comparatively young. This is also suggested by the NIR colors $(J-K=0.7 \ldots 0.9)$ and 
luminosities $\left(M_{J}^{\ddagger}=-8.6 \ldots-9.7\right)$ of bright sources therein which are consistent with a population of young (10-20 Myr) red supergiants (cf. e.g. Elias et al. 1985, Bertelli et al. 1994).

Another conspicuous feature seen at the northeastern part of the BCD is a comparatively blue ( $B-R \approx 0.6$ ) curved strip (region $\mathrm{C}$ ), apparently bending from the NE tip of the LSB component to the north; a counter-feature of this region is probably present at the southern part of the BCD.

The large extent of the SF component, as well as several bright foreground stars in the periphery of Tol 1400-411, render the determination of the structural properties and color of its LSB component difficult. The SBPs (Fig. I.7b) are approximated best with Eq. (I.6) with $(b, q)=(3.0,0.8)$. The mean LSB colors, $J-H=0.42, H-K_{s}=0.28$ and $V-I=0.6-0.7$ can be brought into rough agreement, given the uncertainties discussed in Sect. I.3.5. The GALEV model (see Sect. I.5.4) yields for a metal-poor stellar population forming in a single burst or continuously an age between several $10^{8}$ to a few $10^{9} \mathrm{yr}$ (cf. Fig. I.17). However, these colors might be influenced by ionized gas emission and the younger stellar populations in the NE boundary.

\section{I.4.6 Pox 4}

Despite its moderate metal deficiency $\left(Z_{\odot} / 9.3 \ldots Z_{\odot} / 7.6\right.$; Kunth \& Sargent 1983, Vacca \& Conti 1992), Pox 4 is a relatively luminous $\left(\mathrm{M}_{B}=-18.81\right)$ iI BCG (Méndez \& Esteban 1999). It is accompanied by a faint SF dwarf galaxy, Pox $4 \mathrm{~B}$, at a projected distance of $5 \mathrm{kpc}$ and a velocity difference of $130 \mathrm{~km} \mathrm{~s}^{-1}$. On our NIR images, we do not detect any emission in between Pox 4 and Pox 4 B down to an approximative surface brightness level of $24 J \mathrm{mag} / \square^{\prime \prime}$ (Fig. I.8a).

The optical morphology of Pox 4 is dominated by SF regions and extended ionized gas emission on a spatial scale of $5 \times 2 \mathrm{kpc}$ (cf. Figs. 1 and 2 in Méndez \& Esteban 1999). A substantial color shift due to intense $(E W(H \alpha)=1410 \AA)$ nebular line emission has been observed in the brightest assembly of SF sources M9 (inset of Fig. I.8a) for which Méndez \& Esteban (1999) report a blue ( $-0.8 \mathrm{mag}) U-B$ together with an extremely red ( $+0.7 \mathrm{mag}) B-V$ color. The NIR colors of region M9 $\left(J-H^{\ddagger}=0.28\right.$, $\left.H-K_{s}{ }^{\ddagger}=0.47\right)$ are also suggestive of strong ionized gas contamination. The latter is also reflected on the color profiles (Fig. I.8c), approaching for $R^{*} \lesssim 3^{\prime \prime}(0.7 \mathrm{kpc})$ colors as extreme as $J-H=0.2$ mag together with $H-K_{s}=0.6$ mag.

The SF morphology in Pox 4 is intriguing. Unsharp masking reveals eastwards of region $\mathrm{M} 9$ a ring-like distribution of compact sources with a projected size of $3.2 \times 1.5$ $\mathrm{kpc}$ and a position angle of $\sim 124^{\circ}$. A similar morphology has been reported from optical data by Méndez \& Esteban (1999), who interpreted Pox 4 as a Cartwheel-like galaxy, downscaled by 1-2 orders of magnitude.

The available deep NIR data allow us to detect at faint intensities $\left(\approx 22.5 \mathrm{~J}\right.$ mag/ $\left.\square^{\prime \prime}\right)$ a smooth underlying LSB host galaxy. Its $J_{\text {LSB }}$ profile can be approximated with Eq. (I.6) and a depression parameter $q=0.9$ (Fig. I.8b). From profile decomposition we infer the absolute $J$ magnitude of this stellar host to $-18.2 \mathrm{mag}$, which translates to $M_{B}>-$ 17.2 mag for a $B-J>1$. Thus, despite its high total luminosity, Pox 4 qualifies by the absolute magnitude of its LSB host as a dwarf galaxy. 

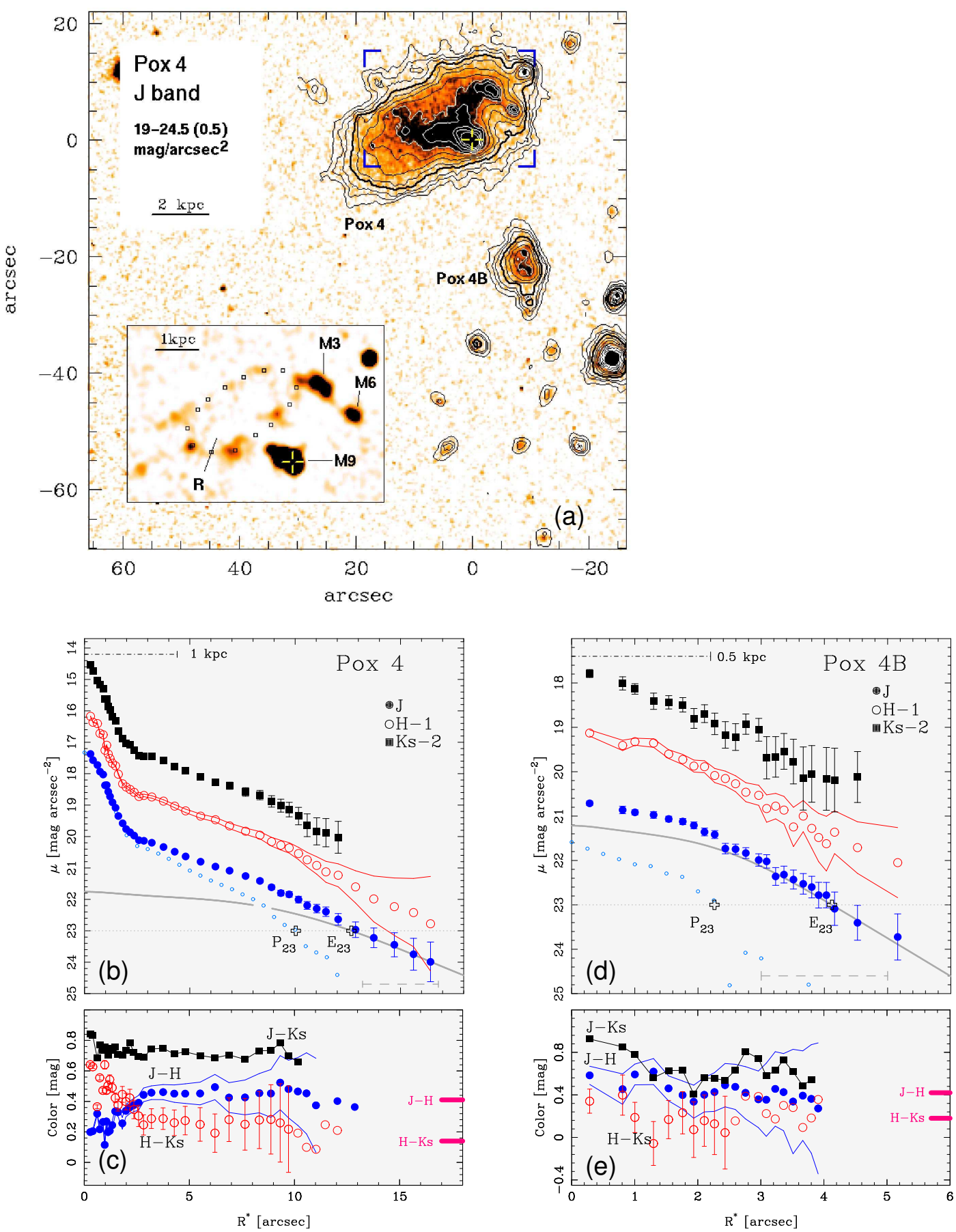

Fig. I.8. Pox 4 and its companion Pox 4B ( $D=46.7 \mathrm{Mpc})$. For explanations of symbols and labels, refer to Fig. I.2. a): $J$ band image and isophotes. The inset shows an unsharp-masked close-up of the SF regions in Pox 4 (area within brackets in the main image). M3, M6 and M9 mark the brightest H II regions referred to as 3, 6 and 9 by Méndez \& Esteban (1999). The ellipse of small boxes marks the ring of SF regions described by the same authors. b)e): Surface brightness and color profiles of Pox 4 (b),c) ) and Pox 4B (d),e) ). The thick grey lines show decomposition fits to the stellar LSB host galaxies by means of modified exponential distributions (see Sect. I.3.2), with $b, q=2.7,0.90$ for Pox 4 and $b, q=2.0,0.80$ for Pox 4B. 
Our SBPs show some similarity to the uncalibrated profiles in Telles et al. (1997) out to $R^{*}=15^{\prime \prime}$. However, the scale length derived here for $10^{\prime \prime} \leq R^{*} \leq 16^{\prime \prime}$ for the LSB component $\left(\alpha=3^{\prime \prime} .8=0.86 \mathrm{kpc}\right)$ is twice as large as that in Telles et al. (1997).

\section{I.4.7 Pox 4 B}

This intrinsically faint $\left(M_{J}=-16.2 \mathrm{mag}\right)$ galaxy has been suggested by Méndez \& Esteban (1999) to have triggered the starburst activity in Pox 4 through a face-on collision. As evidenced by faint $\mathrm{H} \alpha$ emission (Méndez \& Esteban 1999), Pox $4 \mathrm{~B}$ still maintains a mild SF activity. The latter is probably taking place in three compact $(\sim 0$ ". 5$)$ sources, discernible in the central part of the galaxy. Pox $4 \mathrm{~B}$ shows little morphological distortions, except for a slight eastward extension of its LSB component (Fig. I.8a).

The SBPs (Fig. I.8d) of Pox 4 B show for $2^{\prime \prime} \leq R^{*} \leq 5^{\prime \prime}$ an exponential fall-off with an $\alpha \approx 0.29 \mathrm{kpc}$, and a flattening for $R^{*}<2^{\prime \prime}$. We have verified that the latter intensity regime is not due to seeing. For this purpose, we convolved artificial exponential 2D models with the observed $\alpha$ and extrapolated central surface brightness $\left(\mu_{\mathrm{E}, 0} \approx 19.5\right.$ $J$ mag/ $\left.\square^{\prime \prime}\right)$ with a Gaussian kernel matching the PSF of the coadded images $(\approx 0.7$ FWHM). The resulting SBPs deviate only slightly from a pure exponential, even when the FWHM is further degraded by a factor of 2 , and can by no means reproduce the strong flattening we detect in the SBPs of Pox 4B. In addition, a perfect exponential distribution with the $\mu_{\mathrm{E}, 0}$ and $\alpha$ quoted above would yield a $16 \%$ larger luminosity than the one actually measured for Pox 4B.

The available data do not allow us to study the NIR colors in spatial detail. The mean $J-H$ and $H-K_{s}$ of respectively $\approx 0.4$ and $\approx 0.2$ are comparable to those of the LSB component of Pox 4.

\section{I.4.8 Tol 65}

Star formation in this very metal-deficient BCD $\left(Z_{\odot} / 24\right.$; Kunth \& Sargent 1983, Masegosa et al. 1994, Izotov et al. 2001) is taking place mainly in a chain of five compact $\left(1^{\prime \prime} .2 \lesssim \varnothing \lesssim 2^{\prime \prime}\right)$ sources located at the NE part of an irregular, blue LSB envelope (P99). The combination of an extraordinarily blue $J-H^{\ddagger}(\sim 0.1 \mathrm{mag})$ with a red $H-K^{\ddagger}(\approx 0.8 \mathrm{mag})$ color in the surroundings of the brightest SF region e (Fig. I.9a) points to a substantial contribution of ionized gas emission. This is also suggested by the combination of a blue $U-B(\sim-1.05 \mathrm{mag})$ with a moderately red $B-R(\sim 0.4-0.5$ mag) color derived by P99 all over the NE half of Tol 65, several 100 pc away from the opposite tips of the SF chain. Further evidence for intense ionized gas emission is provided by Keck spectroscopy by Izotov et al. (2001), who derived a large $\mathrm{EW}(\mathrm{H} \alpha)$ of $>1000 \AA$ in the SF component of the BCD.

Ionized gas contamination appears to be small midway in the SF chain. The $B-R=0.15 \mathrm{mag}$ (P99), together with $J-H^{\ddagger}$ and $H-K_{s}^{\ddagger}$ of 0.44 and $0.2 \mathrm{mag}$, respectively, observed in region $\mathrm{C}$ are consistent with a single burst stellar age of $\lesssim 100$ Myr. Note that the $J-H$ color in region $\mathrm{C}$ is barely bluer than the average value for 

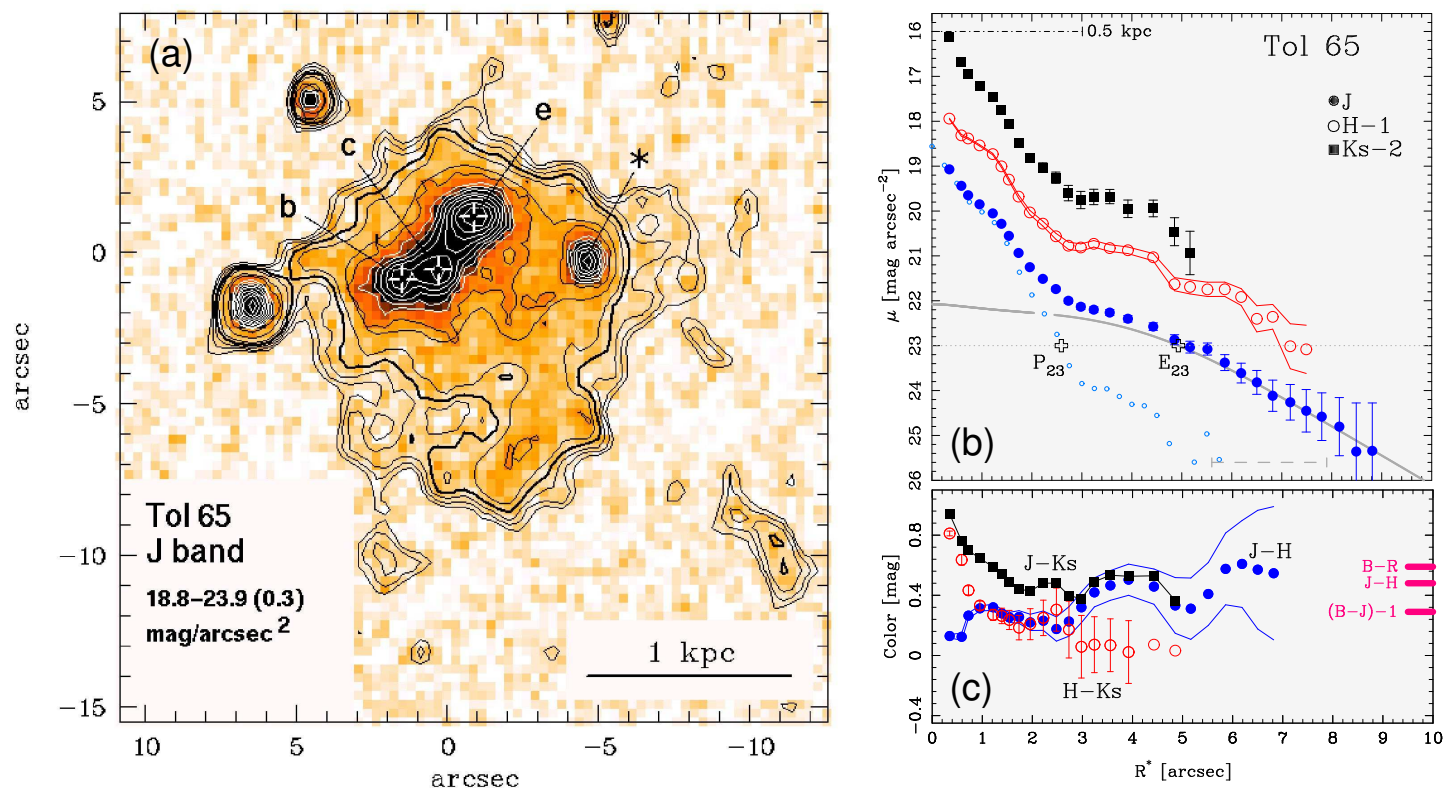

Fig. I.9. Tol $65(D=34.2 \mathrm{Mpc})$. For explanations of symbols and labels, refer to Fig. I.2. a): $J$ band image and isophotes. The sources $\mathrm{b}, \mathrm{c}$ and e along the NE-chain of SF regions are marked, following the notation of P99. The feature marked by an asterisk is a red $\left(J-K_{s} \approx 0.8\right.$ $\mathrm{mag})$ compact source probably not belonging to the galaxy. The faint $\left(\mathrm{m}_{J} \approx 20.8 \mathrm{mag}\right)$ galaxy "G1" (cf. P99) is visible to the SW. b),c): Surface brightness and color profiles. The thick grey line shows a decomposition fit to the host galaxy by means of a modified exponential distribution (see Sect. I.3.2) with $b, q=2.7,0.90$.

the LSB component ( $0.48 \mathrm{mag})$. This is also true for the $H-K_{s}$ color, which could be constrained at $R^{*} \gtrsim P_{23}$ to $\lesssim 0.2 \mathrm{mag}$ (cf. Fig. I.9c).

Deep optical surface photometry $\left(\mu \gtrsim 28.5 \mathrm{~B}\right.$ mag/ $\left.\square^{\prime \prime}\right)$ for Tol 65 has first been presented in P99. These authors found optical SBPs to show an outer exponential regime for $R^{*}>7^{\prime \prime}$ and a flattening relative to the exponential fit inwards of $R^{*} \approx 3^{\prime \prime}$. In order to adequately decompose the optical SBPs, they modelled the LSB component with Eq. (I.6) and a depression parameter $q \gtrsim 0.8$. The type $\mathrm{V}$ profile of the LSB component is better visible in NIR wavelengths, where an even stronger flattening is required ( $q \approx 0.9$ ) to fit the data. The $J$ SBP shows in the radius range $5^{\prime \prime} .0 \leq R^{\star} \leq 7^{\prime \prime} .3$ an exponential intensity fall-off with a scale length $\alpha=0.28 \mathrm{kpc}$, in good agreement with the value derived in P99 ( $\alpha=0.26 \mathrm{kpc}$ ). The average LSB colors of $J-H=0.48 \pm 0.17$, together with the a $B-R \lesssim 0.6 \mathrm{mag}$ and $B-J \lesssim 1.3 \mathrm{mag}$, inferable from the SBPs in P99, are consistent with a stellar age $\lesssim 1 \mathrm{Gyr}$, assuming an instantaneous SF process (cf. Sect. I.3.5).

\section{I.4.9 Tol 1214-277}

This metal-poor $\left(Z_{\odot} / 25\right.$; F01, Izotov et al. 2001) cometary iI BCD undergoes strong SF activity at the NE end of an elongated stellar LSB body with an apparent size of $\approx 6.9 \times 3.4 \mathrm{kpc}$. The brightest SF complex (labelled a in Fig. I.10a) contributes nearly 

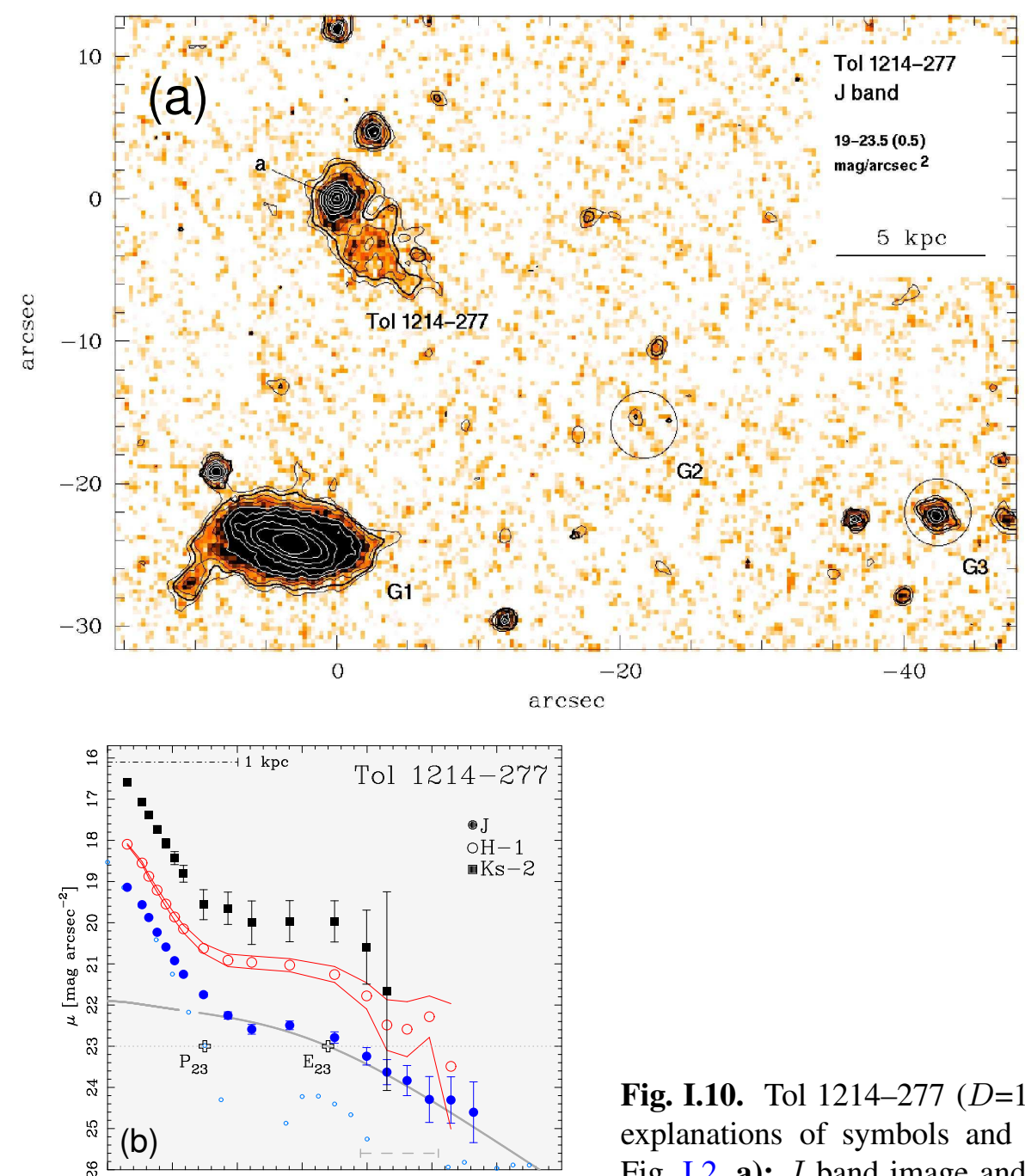

Fig. I.10. Tol 1214-277 ( $D=102.6 \mathrm{Mpc})$. For explanations of symbols and labels, refer to Fig. I.2. a): $J$ band image and isophotes. The bright star-forming complex at the NE edge of the galaxy (a) is marked, along with the faint galaxies G1-G3 (see F01). b),c): Surface brightness and color profiles. The thick grey line shows a decomposition fit to the host galaxy by means of Eq. (I.6) with $b, q=$ $3.3,0.92$.

one half of the BCD’s optical light within the $25 B \mathrm{mag} / \square^{\prime \prime}$ isophote (F01). The $J^{\ddagger}$ magnitude determined for region a, $18.6 \mathrm{mag}$, shows that in the NIR the starburst still provides $\sim 1 / 3$ of the galaxy’s total flux. The blue $J-H^{\ddagger}(-0.02 \mathrm{mag})$ and red $H-K_{s}^{\ddagger}(0.51 \mathrm{mag})$ colors of this region are consistent with a young burst age of $<7$ Myr for the BCD (see also F01).

Only the $J$ band SBP of Tol 1214-277 could be observed with a sufficient quality for a decomposition (Fig. I.10b). Similar to Tol 65, it shows a type V distribution, as already reported from deep optical VLT FORS I data by F01. Fitting Eq. (I.6) to the $J_{\mathrm{LSB}} \mathrm{SBP}$, with the $(b, q)$ parameters fixed to the values derived in F01 $(3.3,0.92)$, we 
obtain a scale length $\alpha=0.53 \mathrm{kpc}$, slightly larger than that in the optical ( $\alpha=0.49 \mathrm{kpc}$; F01).

Fricke et al. (2001) reported for Tol 1214-277 nearly constant $U-B$ and $B-$ $R$ colors of respectively $-0.42 \mathrm{mag}$ and 0.34 mag over an intensity span of $8 \mathrm{mag}$ (colors adapted to the galactic absorption assumed here). Such colors, together with the $J-H=0.4$ mag and $B-J=1.1 \mathrm{mag}$ derived here, are slightly bluer that those of Tol 65, supporting the hypothesis that Tol 1214-277 is a relatively unevolved dwarf galaxy.

\section{I.4.10 Mkn 178 (UGC 6541)}
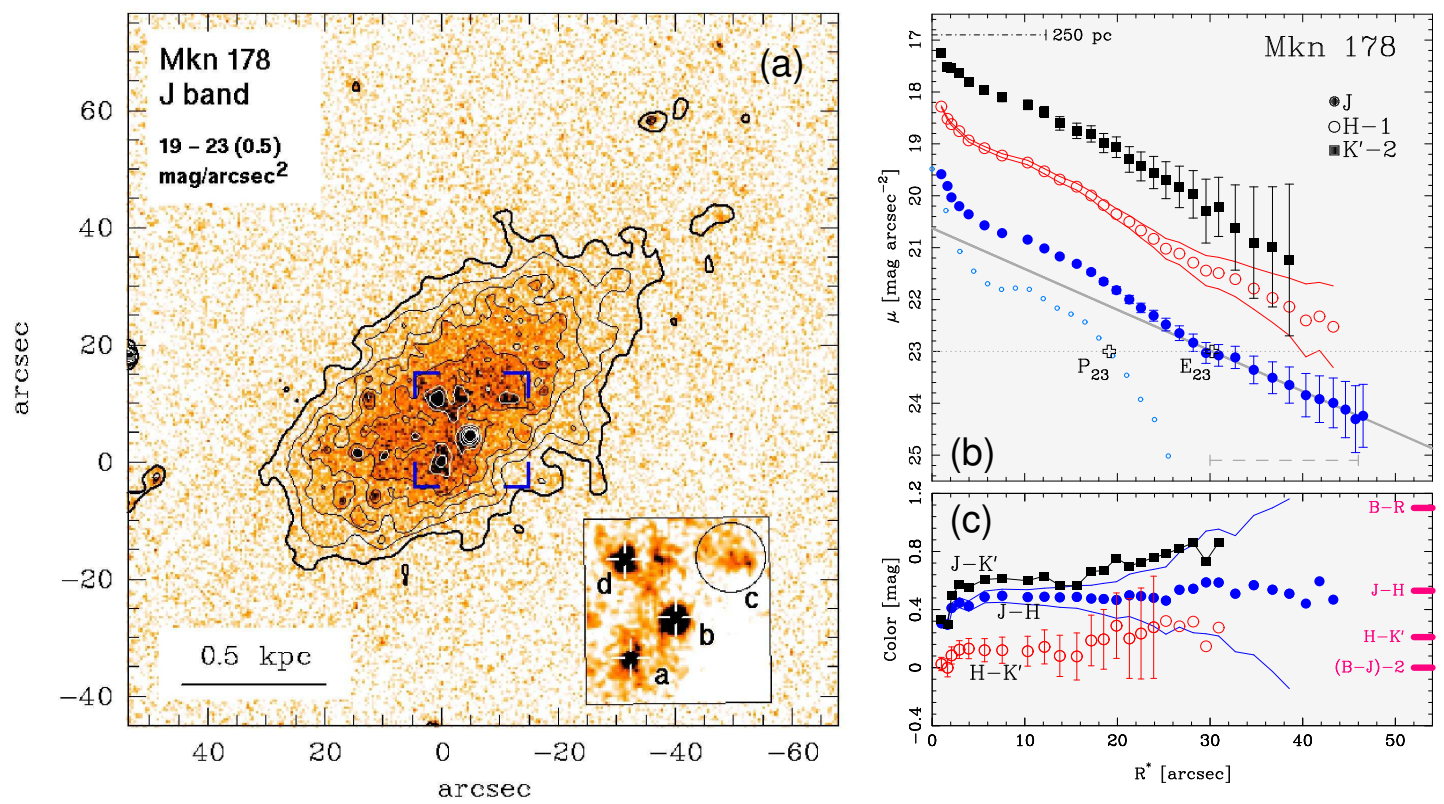

Fig. I.11. Mkn 178 ( $D=4.2 \mathrm{Mpc})$. For explanations of symbols and labels, refer to Fig. I.2. a): $J$ band image and isophotes. The inset shows a close-up of the star-forming regions (delimited by brackets in the main image). The regions a-d described by Gonzalez-Riestra et al. (1988) and Papaderos et al. (2002) are labeled. b),c): Surface brightness and color profiles.

The spectroscopic properties of this intrinsically faint $\left(M_{B}=-13.9\right.$ mag) $\mathrm{iE} \mathrm{BCD,} \mathrm{a}$ member of the CVn cloud I of galaxies (Makarova et al. 1998), have been investigated in e.g. González-Riestra et al. (1988) and Guseva et al. (2000). Optical images reveal a complex morphology in the SF component, notably a pronounced separation of HSB regions into a compact assembly of bright knots to the SE (regions $a$ and $b$ ) and an extended arc-like segregation of fainter sources to the NE. NIR images, on the contrary, show a more regular morphology, with only a few prominent sources in the central part of Mkn 178 (see Fig.I.11a). Region a $\left(m_{J}^{\ddagger}=17.5\right.$ mag; cf. inset in Fig. I.11a) coincides with the optically brightest region in the BCD and is the main locus of active star formation. The brightest NIR source, $\mathrm{b}\left(m_{J^{\ddagger}}^{\ddagger}=16.5 \mathrm{mag}\right)$, is optically faint and shows nearly the same $B-R$ color as the surrounding diffuse emission within the 
plateau component (cf. Papaderos et al. 2002). Sources C and d are immersed within the northern featureless region. The non-detection of an optical counterpart for region $\mathrm{d}$, and the overall optical/NIR morphology of the SF component are suggestive of inhomogeneous, large-scale dust absorption on a spatial scale of $\sim 1 \mathrm{kpc}$. This may cause the apparent separation of the SF component in two large detached complexes and hide knot $d$ in optical wavelengths.

González-Riestra et al. (1988) and Guseva et al. (2000) have shown ionized gas emission to be negligible in region $b(\mathrm{EW}(\mathrm{H} \beta)=24 \ldots 34 \AA)$. By the color excess of $\mathrm{E}(B-V)=0.25$ given in González-Riestra et al. (1988), its observed $B-R$ color (0.67 mag, Papaderos et al. 2002) transforms to $0.28 \mathrm{mag}$, suggesting a single-burst age of $\lesssim 100$ Myr. This is consistent with the colors of $J-H^{\ddagger}=0.24, H-K^{\prime \ddagger}=0.06$ and $B-J^{\ddagger}=1.6$ mag obtained in the present work, if they are de-reddened adopting the same amount of intrinsic extinction. The presence of stellar complexes with an age of the order of $\sim 10^{8} \mathrm{yr}$ is in line with the detection of numerous luminous AGB stars in NIR color-magnitude diagrams (Schulte-Ladbeck et al. 2000), from which these authors infer significant SF activity over the last few $10^{8} \mathrm{yr}$.

NIR SBPs of Mkn 178 (Fig. I.11b) resemble closely the optical ones (Papaderos et al. 2002), showing an extended plateau component ontop a smooth, exponential LSB host galaxy. For the latter we derive a $J$ band scale length of $0.28 \mathrm{kpc}$ and colors $\left(J-H \approx 0.53, H-K^{\prime} \approx 0.2, B-J \approx 2.0\right.$ and $\left.B-R \approx 1.1 \mathrm{mag}\right)$ that consistently indicate an old stellar LSB background.

\section{I.4.11 Mkn 1329 (IC 3589/91, UGC 7790, VCC 1699)}

This relatively metal-rich $\left(Z_{\odot} / 5\right.$, Guseva et al. 2000) cometary iI BCD $\left(\mathrm{M}_{\mathrm{B}} \approx-16.8\right.$, Yasuda et al. 1997) is associated with a group of 11 galaxies (LGG 296, García 1993) within the Virgo Cluster.

As shown in the contrast-enhanced image (inset in Fig. I.12a), numerous irregular concentrations are present in the inner zone of its elongated stellar LSB component, over a projected length of $\sim 3.5 \mathrm{kpc}$. An $\mathrm{H} \alpha$ exposure by Gallagher \& Hunter (1989) shows that SF activity is almost exclusively confined to a bright $\mathrm{H}$ II region (marked with a cross in Fig. I.12a) at the SW end of the high-surface brightness component. The $\mathrm{H} \alpha$ flux derived for that SF region by the latter authors yields, at the distance adopted here, an $\mathrm{H} \alpha$ luminosity of $\approx 1.5 \times 10^{40} \mathrm{erg} \mathrm{s}^{-1}$ and a star formation rate (SFR) of $\sim 0.1 \mathrm{M}_{\odot} \mathrm{yr}^{-1}$. The dominant SF region in Mkn 1329 is identifiable with a compact $(\varnothing \lesssim 300 \mathrm{pc})$, luminous $\left(M_{J}=-14\right)$ NIR source; its colors, $(J-H)^{\ddagger}=0.16$ and $(H-$ $\left.K_{s}\right)^{\ddagger}=0.51$, are probably significantly influenced by nebular emission $(\mathrm{EW}(\mathrm{H} \beta)=276$ Å, Guseva et al. 2000).

The morphology of the SF regions and the high luminosity of the SW complex in Mkn 1329 are typical among cometary iI BCDs. The similarity of these systems with respect to the morphology of their LSB and SF component suggests a comparable evolutionary state/history or common mode of star formation. Propagation of SF activities along those objects' major axes has been proposed and observationally supported by a number of recent studies (cf. Noeske et al. 2000 and references therein). 

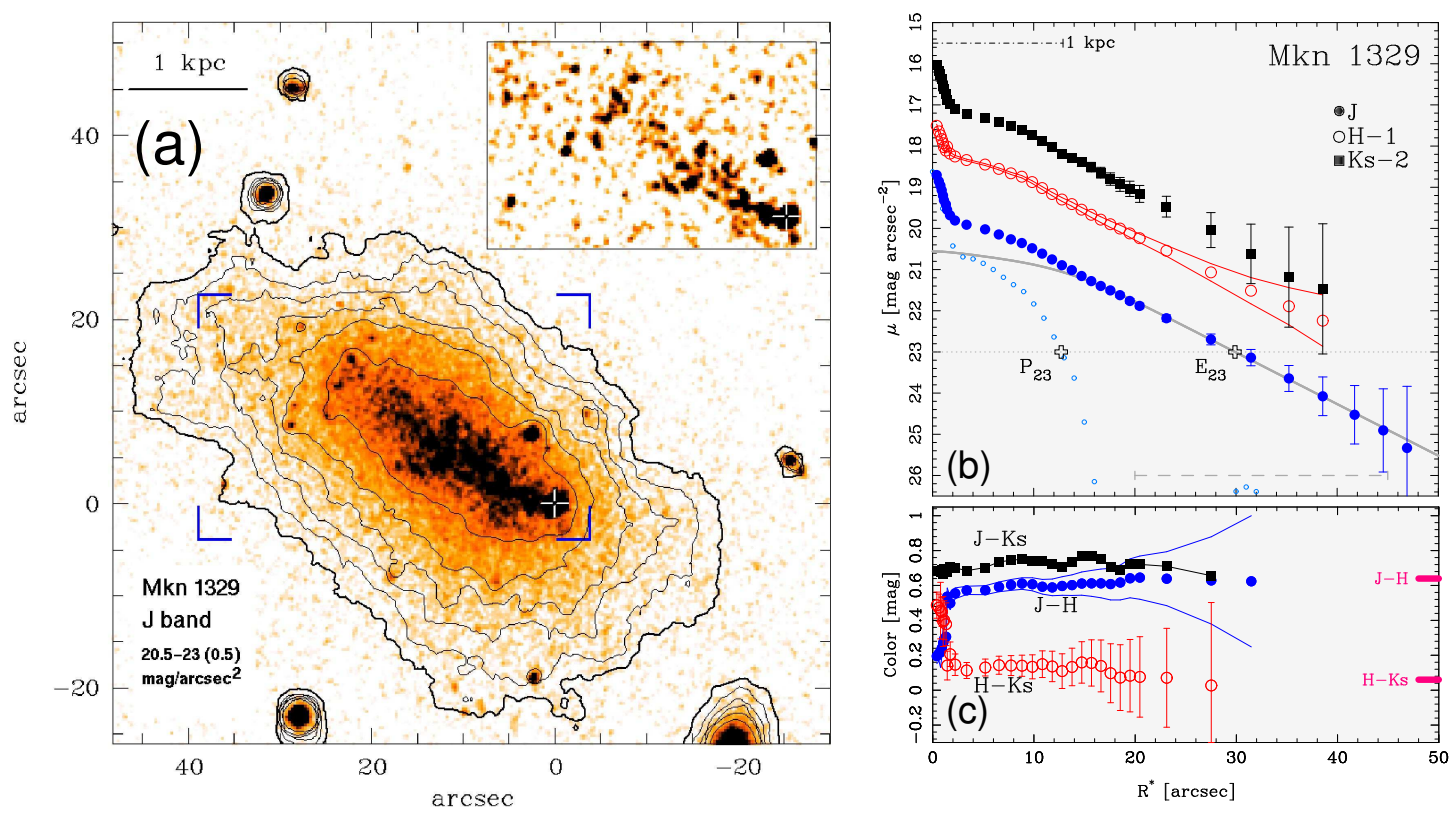

Fig. I.12. Mkn 1329 ( $D=16 \mathrm{Mpc}$ ). For explanations of symbols and labels, refer to Fig. I.2. a): $J$ band image and isophotes. inset: $h b$-transformed image of the central area (delimited by the brackets in the main image), showing the distribution of the bright regions along the major axis. The white cross marks the bright SW star-forming region for orientation. b),c): Surface brightness and color profiles. The thick grey line shows a decomposition fit to the host galaxy by means of a modified exponential distribution, Eq. (I.6), with $b, q=1.6,0.70$.

The SBPs of Mkn 1329 (Fig. I.12b) indicate a moderate central flattening of the LSB component, which can be approximated both by a Sérsic law with $\eta_{\mathrm{LSB}} \approx 0.7$, or a med with $b, q=(1.6,0.7)$ (see Tab. I.2 and Sect. I.3.2). The detection of a moderate central flattening in the LSB component owes to the comparatively small contribution of the superposed younger stellar population in Mkn $1329(\approx 17 \%$ of the total $J$ band light). The NIR colors in the outskirts of Mkn 1329, $(J-H)^{\ddagger}=0.64,\left(H-K_{s}\right)^{\ddagger}=0.06$, suggest an evolved stellar LSB component. Adopting a $B-J$ color characteristic of a few Gyr old stellar population, we can estimate the $B$ band structural parameters from the $\mu_{\mathrm{E}, 0}, \alpha$ and $\mathrm{M}_{\mathrm{LSB}}$ determined from $J$ SBPs. We find that the host galaxy of Mkn 1329 shows, similar to other cometary BCDs (Noeske et al. 2000), structural properties intermediate between extended dIs/dEs and compact $\mathrm{iE} / \mathrm{nE}$ BCDs.

\section{I.4.12 IC4662 (ESO 102-G014, He 2-269)}

This nearby ( $D=2$ Mpc; Heydari-Malayeri et al. 1990) dwarf galaxy has been studied spectroscopically by e.g. Pastoriza \& Dottori (1981), Stasinska et al. (1986), HeydariMalayeri et al. (1990) and Hidalgo-Gámez et al. (2001). The latter authors derived the oxygen abundance in the brighter SF regions $\mathrm{A} \& \mathrm{~B}$ to $Z_{\odot} / 6.5 \ldots Z_{\odot} / 7.6$, respectively. A continuum-subtracted $\mathrm{H} \alpha$ map (Fig. I.13b) from Papaderos et al. (2002) reveals an extended and complex morphology of the ionized gas emission in the upper half of the galaxy, where the $\mathrm{H} \alpha$ emission peaks, and shows a number of shells extending up 

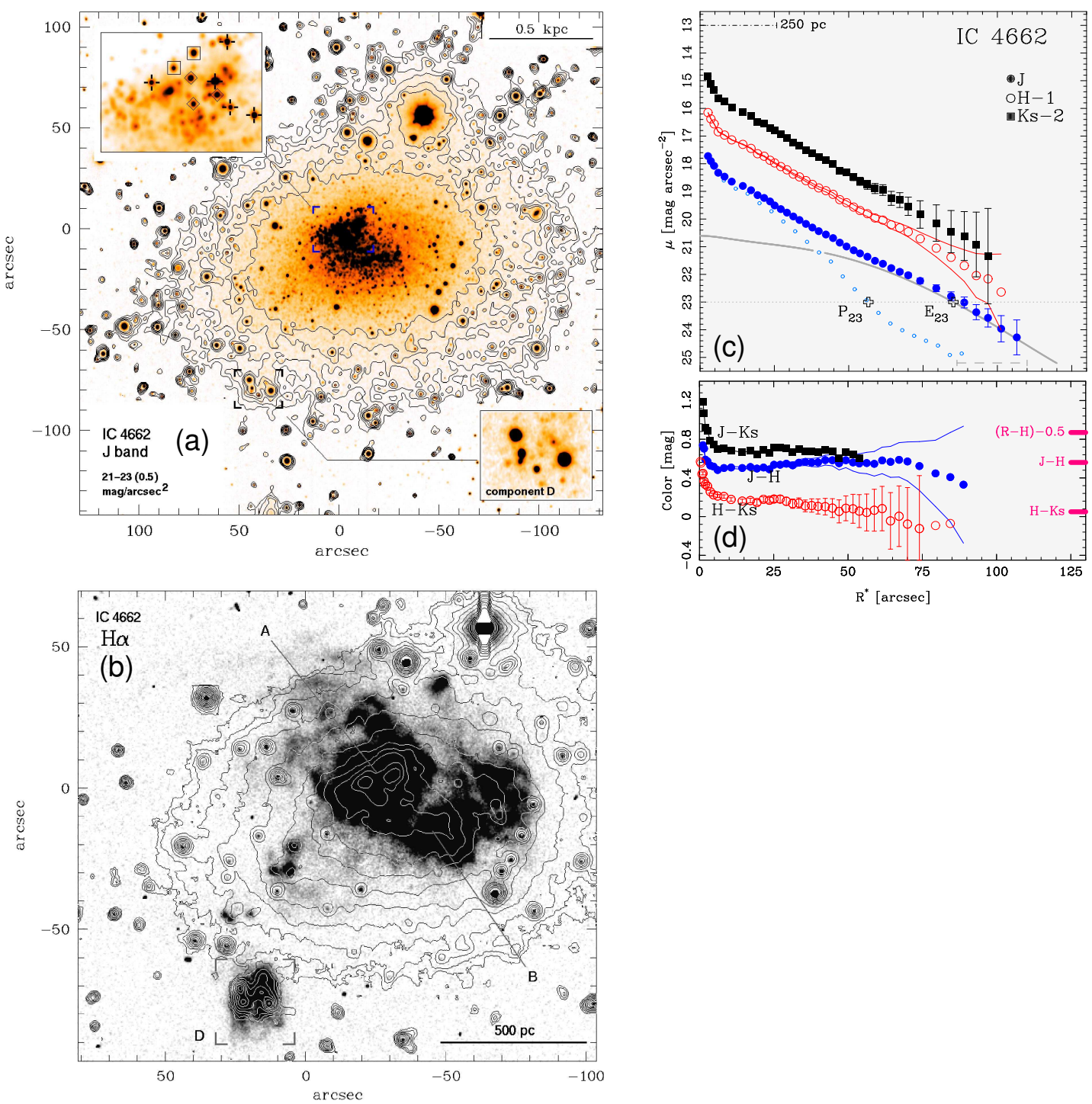

Fig. I.13. IC 4662 ( $D=2 \mathrm{Mpc}$ ). For explanations of symbols and labels, refer to Fig. I.2. (a): $J$ band image and isophotes. The insets show magnified views of the star-forming region $\mathrm{A}$ in the central part of the $\mathrm{BCD}$ and of the D component, about 1!.4 from A. Some bright sources in region A are classified according to Schulte-Ladbeck et al. (2001) into red supergiants ( $J-H \sim 0.75$, crosses), blue supergiants ( $J-H<0.5$, squares), and AGB stars ( $J-H>0.9$, lozenges). (b): Continuum-subtracted $\mathrm{H} \alpha$ map (from Papaderos et al. 2003), showing that all regions (A-D from Heydari-Malayeri 1990) are $\mathrm{H} \alpha$ emitting. (c),(d): Surface brightness and color profiles. The thick grey line shows a fit to the host galaxy using a modified exponential distribution, Eq. (I.6), with $b, q=4.8,0.97$

to $\sim 0.5 \mathrm{kpc} \mathrm{NE}$. Another interesting feature is the extranuclear region $\mathrm{D}$, seen in the outer regions ( $\mu \gtrsim 22 \mathrm{~J} \mathrm{mag} / \square^{\prime \prime}$ ) of IC 4662. Hidalgo-Gámez et al. (2001) find this $\mathrm{H} \alpha$ emitting region to be less metal-rich than the central SF regions (A), and to show a significant recession velocity difference $(250 \pm 150 \mathrm{~km} / \mathrm{s}$, Hidalgo-Gámez et al. 2002) to IC 4662. Our deep images show that region D is not well detached from the main 
body of diffuse $\mathrm{H} \alpha$ emission in IC4662 (contrary to Heydari-Malayeri et al. 1990), but apparently connecting with regions $\mathrm{A} \& \mathrm{~B}$ through a chain of $\mathrm{H} \alpha$ sources.

Hidalgo-Gámez et al. (2002) have proposed that region D may be either a chemically and kinematically distinct complex within IC 4662, or a close companion object. The latter possibility is particularly interesting in view of the hypothesis that very close, gas-rich dwarf companions might be conceivable triggering agents of starburst activity in BCDs (Taylor et al. 1995, Pustilnik et al. 2001, Noeske et al. 2001).

From the available data, we resolve a wealth of morphological information in the central part (Fig. I.13a) of IC 4662, notably the head-tail morphology of the starburst. Luminosities and colors of the brightest point sources in region A are in agreement with those of red supergiants, supporting the results by Heydari-Malayeri et al. (1990), as well as of blue supergiants and AGB stars (cf. the upper left inset in Fig. I.13a; see Schulte-Ladbeck et al. 2001 for color limits separating the latter classes of giant stars).

As IC 4662 is located at low galactic latitude $\left(-17.8^{\circ}\right)$, photometric studies of its LSB component are complicated by the dense foreground Galactic stellar field. Also residuals in the removal of the NW bright star may affect the photometry for faint isophotal levels. A tentative exponential fit to the $J$ SBP yields for $R^{*}>65^{\prime \prime}$, i.e. outside significant nebular emission, a scale length of $\sim 0.21 \mathrm{kpc}$. However, inspection of the $J_{\text {LSB }}$ profile shows that the BCD follows a type $\mathrm{V}$ distribution, fitted best with Eq. (I.6) with a central surface brightness of $16.8 J \mathrm{mag} / \square^{\prime \prime}$, a scale length of $\sim 150 \mathrm{pc}$ and a depression parameter as large as $q \approx 0.97$. If so, the starburst contributes $\sim 50 \%$ of the total $J$ light of the BCD. The structural properties of its LSB host, as well as its intense and spatially extended SF activity place IC 4662 in the range of BCDs, making it probably one of the closest BCDs known.

\section{I.4.13 UM 488 (Mkn 1304, SBS 1139+006, UGC 6665)}

This is a distant ( $D \approx 76 \mathrm{Mpc}$, Mirabel \& Sanders 1988) blue compact galaxy, known to be relatively metal-deficient ( $Z_{\odot} / 8.5$; Masegosa et al. 1994, Izotov \& Thuan 1999). Despite its resemblance (Fig. I.14a) to cometary iI BCDs, UM 448 does not qualify as a dwarf due to its intrinsic luminosity $\left(M_{B}=-19.7 \mathrm{mag}\right)$ and large linear extent (major axis length $\sim 25 \mathrm{kpc}$ ). Active star formation is taking place within an extended (diameter $\sim 7 \mathrm{kpc}$ ) high surface brightness region at the NE part of the galaxy, showing some fainter SW extension. The starburst component contributes $\approx 70 \%$ of the $J$ band light. Guseva et al. (2000) estimated the starburst luminosity to be powered by $1.6 \times 10^{6}$ $\mathrm{O}$ stars (value referring to the distance assumed here), $0.6 \%$ of which undergoing their Wolf-Rayet phase. Studies by Sage et al. (1992) indicate an ongoing SFR of $21 \mathrm{M}_{\odot}$ $\mathrm{yr}^{-1}$, and a large reservoir of molecular gas amounting to $2.8 \times 10^{9} M_{\odot}$ or $\sim 1 / 2$ of the H I mass $\left(5.8 \times 10^{9} M_{\odot}\right.$; Mirabel \& Sanders 1988) of UM 448.

On unsharp-masked images, the SF region splits into 3 high-surface brightness entities (see the inset in Fig. I.14a), the brightest one being the reddest $\left(J-H^{\ddagger}=0.7 \mathrm{mag}\right.$ ) whereas the fainter regions $\mathrm{b}$ and $\mathrm{c}$ are slightly bluer $\left(J-H^{\ddagger} \approx 0.5 \mathrm{mag}\right)$. The SBPs (Fig. I.14b) show an exponential decay for $R^{*}>15^{\prime \prime}$ with a $J$ band scale length of 3.8 $\mathrm{kpc}$, somewhat smaller than the value $\sim 5.2 \mathrm{kpc}\left(14^{\prime \prime}\right)$ inferred in Telles et al. (1997). 

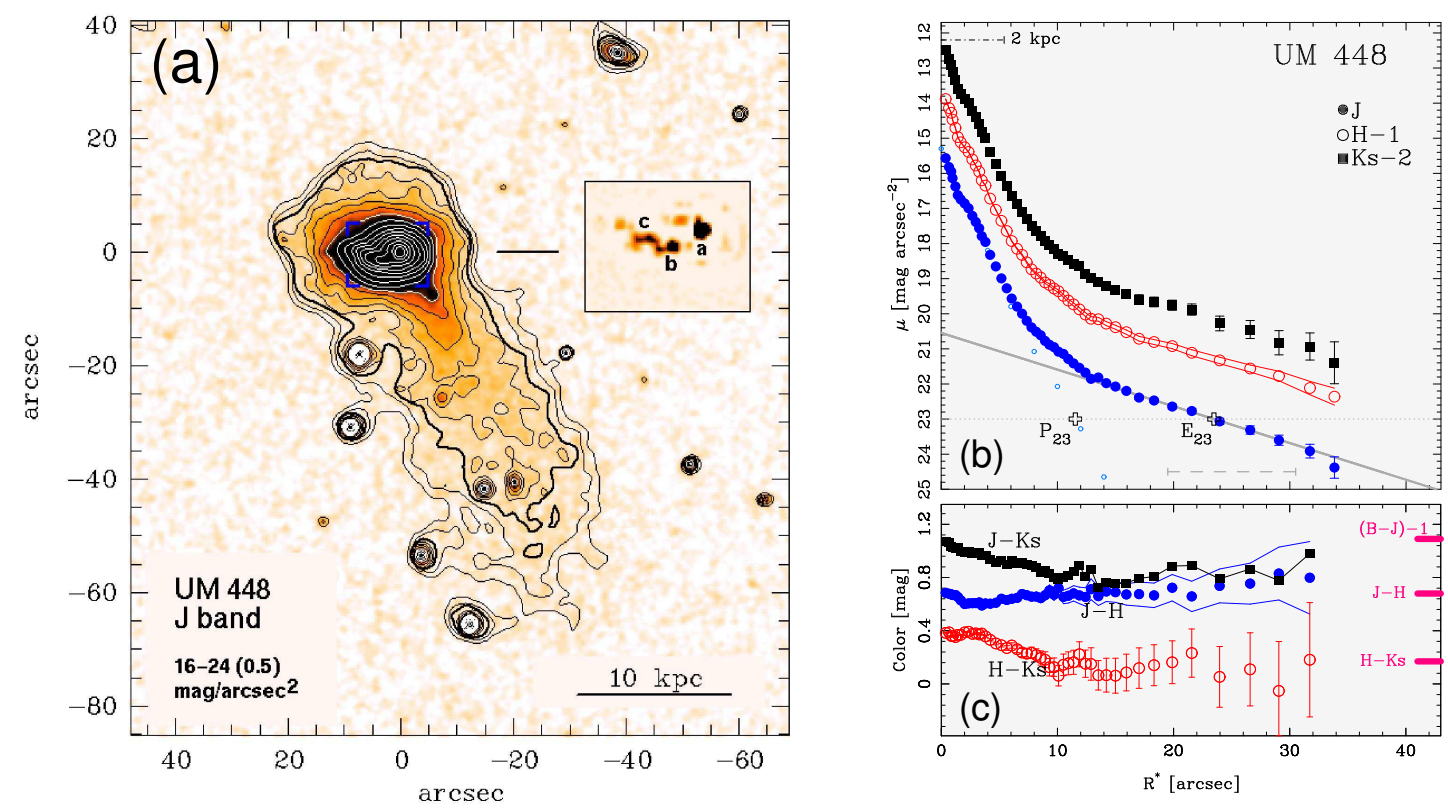

Fig. I.14. UM 448 ( $D=76.1 \mathrm{Mpc})$. For explanations of symbols and labels, refer to Fig. I.2. a): $J$ band image and isophotes. The inset shows a contrast-enhanced close-up of the starforming regions (delimited by brackets in the main image); individual knots (a-c) are labeled. b),c): Surface brightness and color profiles.

\section{I.5 Discussion}

\section{I.5.1 The shape of NIR surface brightness profiles}

The SBPs derived for the sample BCDs in Sect. I.4 bear close resemblance to those typically inferred from optical broad-band data (cf., e.g., P96a, Telles et al. 1997, Marlowe et al. 1997, Doublier et al. 1997,1999, Cairós et al. 2001a). In most cases, SBPs show in their outer part an exponential intensity decrease and red, nearly constant colors. This outermost SBP part is attributable to the underlying LSB host galaxy which, except for a few rare cases, shows clear evidence for a several Gyr old stellar population. At intermediate and small radii the emission of a BCD is dominated by the younger stellar component. In the main class of $\mathrm{iE} \mathrm{BCDs} \mathrm{(LT86)} \mathrm{its} \mathrm{luminosity} \mathrm{output}$ is reflected on two conspicuous SBP components.

1) At small radii $\left(R^{*} \$ 100 \mathrm{pc}\right)$, a feature commonly observed in optical and NIR SBPs is a central intensity excess. This is seen in e.g., the $J$ SBP of Tol 3, Mkn 178, Mkn 1329, Haro 14 (our sample) or Mkn 36 and UM 462 (C02b). This component is due to the brightest and typically youngest stellar assembly. In most cases, this narrow innermost excess is due to one and the same region in both optical and NIR wavelengths. An exception is presented by Mkn 178, where the brightest region in the NIR is offset from that in the optical by $\sim 150 \mathrm{pc}$, or by the BCD Mkn 35 in the sample of $\mathrm{C} 02 \mathrm{~b}$.

2) The second SBP feature has been referred to as plateau in P96a. It shows a nearly constant or slowly decaying intensity, and extends typically out to $\mu \sim 24 B$ mag/ $\square$ ". 
SBPs with a prominent plateau on top a more extended exponential LSB envelope can in general not be adequately fit by a simple function (e.g. a Sérsic profile) over their whole intensity span. A meaningful decomposition of such SBPs is only possible when an extra component (e.g., a Sérsic distribution with $\eta \leq 1$ ) is introduced in order to fit the plateau (P96a, Cairós et al. 2001a). There is, so far, no observational support for the plateau being a dynamically distinct or even interaction-induced stellar entity with nearly constant $\mathcal{M} / \mathcal{L}$. Quite contrary, appreciable color gradients $(\lesssim 1.5 B-R$ mag $\mathrm{kpc}^{-1}$ ) in the HSB part of BCDs indicate that the plateau light is mainly due to a young and moderately evolved stellar population. P96b have shown that a conspicuous plateau in the SBPs of many iE BCDs can naturally result from the superposition of diffuse and compact SF sources with varying luminosity and galactocentric distance on a more extended, exponential LSB component. Conversely, the plateau is nearly absent in the intrinsically brighter $\mathrm{nE}$ BCDs, where most of the starburst light originates from the nuclear region of the BCD. The overall SBPs of these systems show frequently a concave shape, fitted satisfactorily by Eq. (I.8) with a Sérsic exponent $\eta \geq 1$, and sometimes resemble a de Vaucouleurs profile.

Discerning the formation history of the stellar populations memorized in the plateau light poses a challenge for surface photometry and CMD studies, as nebular line emission (e.g. Tol 65; Papaderos et al. P99, Izotov et al. 2001, Tol 1214-277; F01) and patchy dust absorption (Tol 3, Mkn 178; this paper, Mkn 33 and Mkn 35; C02b, I Zw 18; Cannon et al. 2002) may both hamper standard age-dating techniques. In addition, an important and mostly overlooked source of systematic uncertainties in the determination of colors or EWs within the plateau stems from the unknown line-of-sight contribution of the underlying old LSB component. As pointed out in Sect. I.3.3, the latter may redden colors of compact stellar clusters in the SF region by up to a few tenths of magnitude. Corrections of this order have also been reported for the iE BCDs Mkn 370 and Mkn 178 by Cairós et al. (2002a) and Papaderos et al. (2002), respectively. Evidently, for such a correction one has to assume a model for the intensity distribution of the LSB component just beneath the SF regions (i.e., for $R^{*} \lesssim P_{23}$ ). It is a common practice to extrapolate the exponential slope of the LSB periphery of BCDs all the way to $R^{*}=0^{\prime \prime}$. However, the universal validity of this procedure for BCDs has been questioned in, e.g., P96a. These authors discussed observational evidence for type $\mathrm{V}$ profiles in BCDs and proposed the alternative fitting formula Eq. (I.6) to approximate such convex distributions. Alternatively, $\mathrm{C} 02 \mathrm{~b}$ discuss cases where the LSB host galaxy shows a concave profile which is better fit by a Sérsic model with an exponent $\eta>1$. The photometric structure of the LSB host of BCDs will be discussed in the light of the present NIR data in the next section.

\section{I.5.2 Hints to a centrally flattening exponential distribution in the underlying stellar LSB component?}

Nine of the BCDs included in our sample (Tab. I.2) show signatures of a type $\mathrm{V}$ profile in their LSB hosts. This is because an exponential law, or any Sérsic distribution with $\eta>1$, fitted to the outer part of their $J$ SBPs, predicts at intermediate to small radii a 


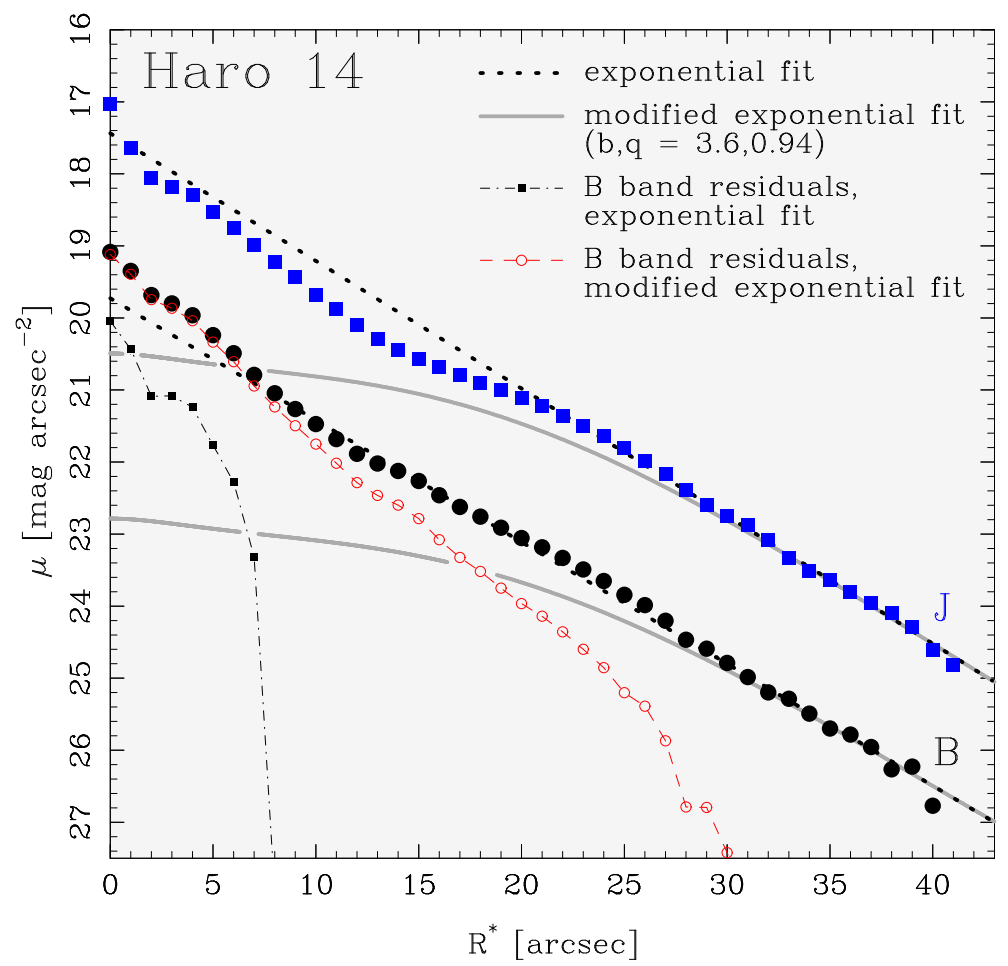

Fig. I.15. Decomposition of the $B$ band SBP of Haro 14 (filled circles) assuming for its LSB host a pure exponential model (dotted line) or a modified exponential distribution (Eq. I.6; thick gray curve) with the flattening parameters $(b, q)=(3.6,0.9)$ derived in the $J$ band. The surface brightness distribution of the star-forming component, as obtained by subtraction of the respective LSB model from the $B$ SBP is illustrated by the filled and open interconnected symbols (see discussion in the text).

higher intensity than the observed value (cf. Sect. I.3.2). Of course, pure exponential fits to $J_{\text {LSB }}$ profiles cannot always be definitely ruled out within the $1 \sigma$ uncertainties. However, such fits would either overestimate the central intensity of the stellar LSB host, hence underestimate the luminosity fraction of the SF component (cf. Sect. I.5.2) or systematically overestimate the $J$ band exponential scale length, thus imply an implausibly large $B-J$ color gradient for the old underlying LSB population.

The limited size of our present sample does hardly allow to estimate the frequency of type $\mathrm{V}$ profiles in BCDs. This issue will be addressed in a forthcoming paper of this series, focussing on the complete NIR sample. However, the evidence gathered so far (see also Sect. I.3.2) strongly supports the idea that a substantial fraction of BCDs shows type $\mathrm{V}$ profiles in its LSB host galaxy. The rare detection of type $\mathrm{V}$ profiles in BCDs in previous optical studies can be explained by the fact that in those wavelengths extended starburst emission overshines the LSB host within typically its inner $\sim 2$ exponential scale lengths (P96b, Noeske 1999, Cairós 2000, Cairós et al. 2001a). As a result, a type $\mathrm{V}$ profile in $\mathrm{iE} / \mathrm{nE} \mathrm{BCD}$ could be detected only when a significant depression of the exponential LSB slope occurs for $R^{*}>2 \alpha$, i.e. if $b>2$ in Eq. (I.6). NIR observations do not overcome, but alleviate the problem of severe light pollution 
by the burst, proving in many cases crucial for disentangling a pure exponential from a type $\mathrm{V}$ distribution in the underlying LSB galaxy.

This is illustrated in Fig. I.15 on the example of the iE BCD Haro 14. Its $B$ SBP is roughly exponential out to $R^{*}=40^{\prime \prime}(\mu \sim 27 B$ mag/ $\square$ "), suggesting that the exponential slope seen in the LSB periphery continues all the way to $R^{*}=0^{\prime \prime}$. In that case, one is tempted to conclude that the ongoing burst gives rise to a modest luminosity enhancement, only. Indeed, subtraction of the exponential LSB model from the SBP suggests that SF sources are all confined within $R^{*}=8^{\prime \prime}$ (cf. the small interconnected squares in Fig. I.15) and that they account for no more than $7 \%(\geq 16.5 \mathrm{mag})$ of the $B$ light of the BCD. Evidently, this conclusion is hardly compatible with the extended morphology of the SF component and copious $\mathrm{H} \alpha$ emission of Haro 14 (cf. Marlowe et al. 1997, Doublier et al. 1999).

One arrives at a quite opposite conclusion on inspection of the NIR profiles. Figure I.15 shows that a pure exponential law falls short of fitting the $J$ SBP inwards of $R^{*}<20^{\prime \prime}$; a plausible fit to the NIR data must invoke a significant flattening of the exponential slope inside $\gtrsim 3$ scale lengths of the LSB component. An adequate fit to the $J_{\mathrm{LSB}}$ is best achieved with a med (Eq. I.6) with $(b, q)=3 \cdot 6,0.94$. Such a strongly flattened exponential LSB model yields a stellar mass by a factor $\sim 3$ smaller than that of a purely exponential profile and implies that the starburst (small interconnected circles in Fig. I.15) contributes $\sim 60 \%$ of the $B$ light within the Holmberg radius, i.e. more than a factor of eight larger than the value inferred before. The isophotal $B$ magnitude within $25 B$ mag/ $\square^{\prime \prime}$ is then increased by $\sim 1.1 \mathrm{mag}$ due to the starburst. This value is still compatible to the mean value of 0.75 mag deduced for BCDs in P96b and Salzer \& Norton (1999).

The considerations above show that the choice of the model for the LSB emission may have far-reaching implications to our view about the SF amplitude and photometric fading of BCDs. The usual assumption of a purely exponential LSB model may, in some cases, strongly underestimate the luminosity fraction of the young stellar population, and lead to the conclusion that a "burst" is merely a minor luminosity enhancement in the lifetime of a BCD. If, on the contrary, the LSB emission is assumed to follow a type $\mathrm{V}$ profile, then the estimated luminosity of the superimposed young stellar population may increase by more than a magnitude.

Consequently, different LSB models imply different amounts of photometric fading of a BCD once SF activities have terminated. This has to be borne in mind when discussing evolutionary links between BCDs and other dwarf galaxies, as well as a possible fading of luminous blue compact galaxies at medium redshift to local spheroidals (cf. e.g. Guzmán et al. 1998). The assumption of a type V profile for the LSB host galaxy will result into a, typically, by $<1$ mag fainter total absolute magnitude $M_{\mathrm{LSB}}$. However, it will not change the extrapolated central surface brightness $\mu_{\mathrm{E}, 0}$ and exponential scale length $\alpha$ of the LSB host galaxy of a BCD. Therefore a frequent occurrence of type V LSB profiles is not expected to significantly change the systematic difference between BCDs and other types of dwarf galaxies on the $\mu_{\mathrm{E}, 0}-M_{\mathrm{LSB}}$ and $\log (\alpha)-M_{\text {LSB }}$ parameter space (P96b, Marlowe et al. 1997, Salzer \& Norton 1999, Papaderos et al. 2002). 
A dedicated investigation of the frequency and origin of systematic deviations from the exponential law (e.g. a type $\mathrm{V}$ profile, or Sérsic profile with $\eta>1$ ) in the stellar LSB host of BCDs is apparently of great interest. A first step towards such studies is to test the suitability of different empirical functions in approximating the observed LSB intensity distribution. This issue will be briefly discussed in the following section.

\section{I.5.3 Fitting the LSB component}

Here we wish to check whether a type $\mathrm{V}$ profile, approximated by Eq. (I.6), can be described equally well by a Sérsic law. As none of the sample BCDs shows a concave shape in its LSB component, we restrict ourselves to the discussion of Sérsic profiles with $\eta \leq 1$; such profiles appear to be common among intrinsically faint dwarf galaxies (Sect. I.3.2).

For simplicity, we fit in Fig. I.16 a Sérsic profile (thin dashed line) to the med model (heavy line, labelled 4) obtained for the LSB component of Haro 14 in Sect. I.4. Distributions 2 and 3 illustrate med profiles with equal extrapolated central surface brightness $\mu_{\mathrm{E}, 0}\left(17.46 \mathrm{~J} \mathrm{mag} / \square^{\prime \prime}\right)$ and exponential scale length $\alpha(0.37 \mathrm{kpc})$ as 4 , but differing degrees of flattening $(q)$ with respect to the pure exponential $(q=0)$ model 1. Distributions 2 through 4 assume flattening parameters of $(b, q)$ of $(1.2,0.4),(2.4,0.8)$ and $(3.6,0.94)$, respectively.

The med 4 can be approximated best by a Sérsic profile with a central surface brightness $\mu_{\mathrm{S}, 0}=20.45 \mathrm{~J} \mathrm{mag} / \square^{\prime \prime}$, a scale length $\beta \approx 1.14 \mathrm{kpc}$ and an exponent $\eta=$ 0.57 . Note that the deduced $\mu_{\mathrm{S}, 0}$ compares well to the actual central surface brightness of the LSB host galaxy

$$
\mu_{\mathrm{E}, 0}+2.5 \log \left(\frac{1}{1-q}\right) \approx 20.5 \mathrm{mag} / \square^{\prime \prime},
$$

implied by the med model.

At a first glance, a Sérsic law reproduces nicely a med distribution over a radius range of nearly 10 scale lengths $\alpha$. However, the lower panel of Fig. I.16 shows that the residuals between either model, although small ( $\leqslant 0.2 \mathrm{mag}$ ), are systematic. This is also true for all med profiles considered here, when Sérsic models are fitted over a sufficiently large radius $(>5 \alpha)$. In all cases, a Sérsic model was found to approximate well the inner portion of a med profile (e.g. within $\sim 1.5$ cutoff-radii $b \alpha$ ) but it generally failed to simultaneously account for the exponential intensity fall-off of such a distribution at larger radii. Another drawback of Sérsic models when fitted to non-genuine Sérsic distributions is that the solution can vary considerably, depending on the fitted radius range. For instance, a Sérsic fit to the med 4 (Fig. I.16) for $<23.5 \mathrm{~J} \mathrm{mag} / \square^{\prime \prime}$, or within $5 \alpha\left(\sim 30^{\prime \prime}\right)$ yields an $\eta=0.45$, significantly lower than the value above. In that respect it is worth noting that a Sérsic exponent $\eta<0.5$ reflects a central minimum in the radial luminosity density distribution of the LSB component (P96a). The stability of the shape parameter $\eta$ has been explored in detail in $\mathrm{C} 02 \mathrm{~b}$; these authors remarked that a Sérsic fit can be very uncertain for concave SBPs, where the exponent $\eta$ can vary 
by up to an order of magnitude, depending on the fitted range and the radial sampling of points in the profile.

Another objection to the view that Sérsic models offer a robust tool to systematize the structural properties of dwarfs comes from the degeneracy of the shape parameter $\eta$ vs. the pseudo-scale length $\beta$. A fit of Eq. (I.8) to the med distribution 2 yields an $\eta=0.94$ and a $\beta \approx 0.44 \mathrm{kpc}(>\alpha)$. As for profile 3 , it is best approximated with an $\eta=0.75$ and $\beta \approx 0.72 \mathrm{kpc}(2 \alpha)$. We see that while the Sérsic exponent $\eta$ decreases with increasing degree of flattening, i.e. as we go from distribution 2 towards 4 , the corresponding scale length $\beta$ increases from $0.44 \mathrm{kpc}$ to $1.15 \mathrm{kpc}$. Actually, the exponential scale length $\alpha$ of the med profiles considered here is nowhere recovered by fitting a Sérsic model. Instead, one is left with the pseudo-scale length $\beta$, which by itself alone carries no quantitative information on the structural properties of the LSB component.

Obviously, the scale length $\beta$ is only meaningful in connection with $\eta$. However, these two quantities are rendered impractical for a systematic study of the LSB component by their strong non-linear coupling (see discussion in e.g. Young \& Currie 1994, Cellone \& Buzzoni 2001), and dependence on the fitting procedure (see above). Furthermore, observational uncertainties connected with, e.g., the sky subtraction (see e.g. Cellone \& Buzzoni 2001, C02b), filtering of images prior to surface photometry, and imperfect removal of background sources may also skew the $\beta$ vs. $\eta$ parameters in a hardly predictable manner, making Sérsic fits to the outer part of SBPs a hazardous procedure $(\mathrm{C} 02 \mathrm{~b})$. These problems are probably not worrisome in studies of early type galaxies, i.e. systems with little morphological distortions and a nearly constant $\mathcal{M} / \mathcal{L}$. In irregular SF galaxies, however, the derived Sérsic parameters depend on subjective choices in the data processing (see above), profile fitting and, equally important, on the profile extraction methods themselves. It is conceivable that the latter circumstances imprint the Sérsic parameters $\left(\mu_{\mathrm{S}, 0}, \beta, \eta\right)$ inferred recently for a sample of luminous BCGs by Bergvall \& Östlin (2002). We believe that the extremely large $\eta_{\mathrm{LSB}}$ exponents they derive (up to 20) should be regarded formal only, rather than a manifestation of an extraordinarily dense Dark Matter halo which, as advocated by Bergvall \& Östlin (2002), dominates the stellar dynamics of a BCD.

Such considerations call for deeper photometric studies of the LSB component of BCDs, and in dwarf galaxies in general. A crucial question is whether or not the LSB host galaxy of these systems shows a perfectly exponential fall-off in its outer part, continuing several scale lengths beyond a cutoff radius $b \alpha$ (cf. Eq. I.6). Should that be the case, then no Sérsic model fit to the LSB emission can correctly recover the exponential scale length $\alpha$ and will not be free of ambiguity. Alternatively, if subsequent studies show that the LSB profiles deviate systematically from the exponential law at all radii, then a Sérsic profile might be the preferable fitting law, albeit the drawbacks discussed above. An ultimate check requires deep surface photometry, allowing to derive SBPs out to $\sim 10 \alpha$ with an accuracy better than $0.2 \mathrm{mag}$. As long as tests of this kind await to be done, we argue, in agreement to $\mathrm{C} 02 \mathrm{~b}$, that a pure (Eq. I.5) or modified exponential (Eq. I.6) fitting formula should be preferably used to fit the underlying LSB component of BCDs. 

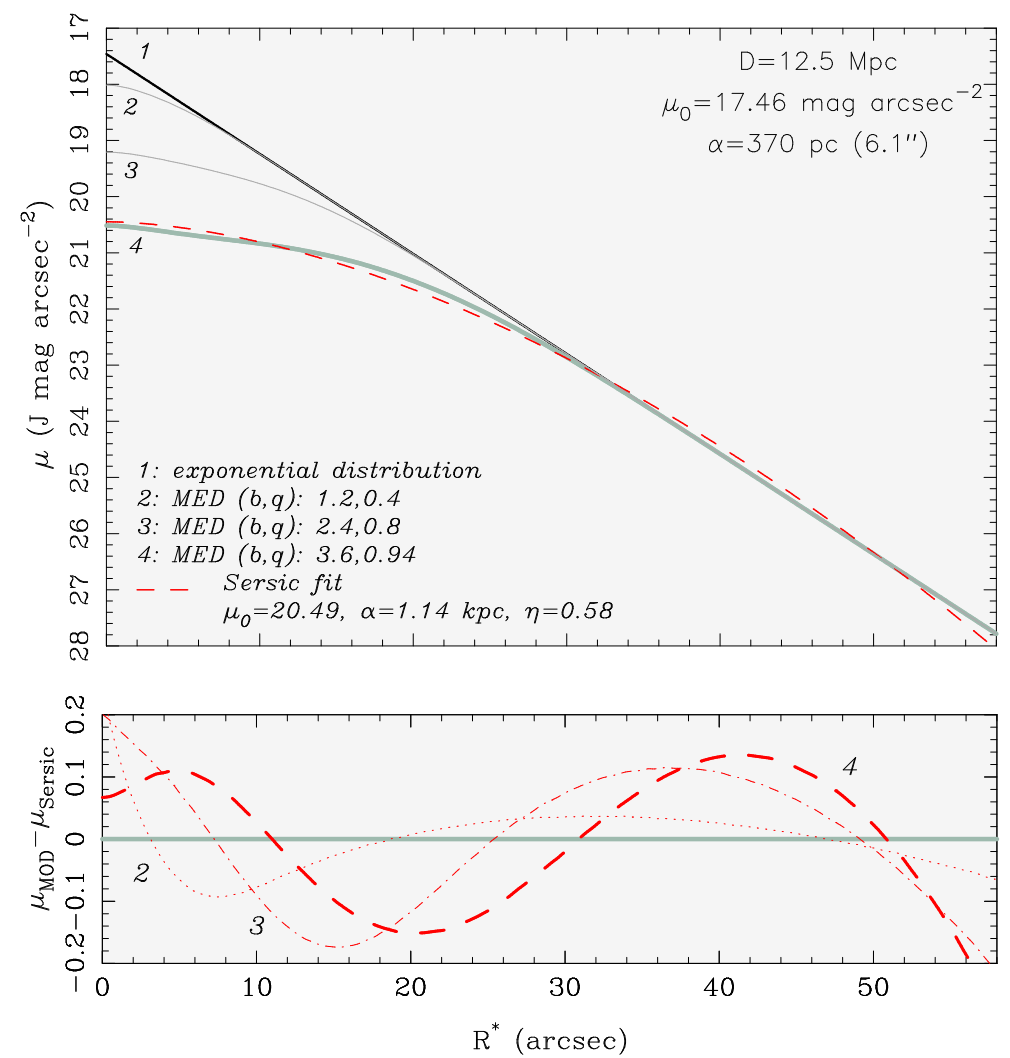

Fig. I.16. upper panel: Fit to the LSB component of Haro 14 (thick gray curve, labelled 4) assuming an inwards flattened exponential distribution (med, Eq. I.6) with the extrapolated central surface brightness $\mu_{\mathrm{E}, 0}$ and exponential scale length $\alpha$ indicated at the upper-right, and flattening parameters $(b, q)=(3.6,0.94)$. Distributions 2 and 3 correspond to a med with equal $\mu_{\mathrm{E}, 0}$ and $\alpha$ but different $(b, q)$, and 1 describes a pure exponential distribution $(q=0)$. The dashed curve shows a fit to distribution 4 using a Sérsic fitting law (Eq. I.8). lower panel: Residuals between the assumed med distributions 2 through 4 (upper panel) and Sérsic fits.

\section{I.5.4 The colors of the stellar LSB host galaxy}

The available NIR data allowed not only for the detection and structural analysis of the LSB component for all sample BCDs, but also for the determination of at least one NIR color in the LSB host (Sect. I.3.5).

The $B-J$ vs. $J-H$ and $H-K_{s}$ vs. $J-H$ LSB colors (Tab. I.3), whenever available, are displayed in Fig. I.17. For comparison, color predictions from the GALEV evolutionary synthesis model (Schulz et al. 2002; Anders, Bicker \& Fritze - v. Alvensleben 2002, priv. comm.) are overlaid in both panels, calculated for stellar populations with metallicities of $Z_{\odot} / 50$ and $Z_{\odot} / 2.5$, formed either in an instantaneous burst or continuously with a constant SFR. As evident from Fig. I.17, NIR colors ( $J-H$ vs. $H-K_{s}$ ) show little evolution for old stellar populations ( $\leqslant 0.1 \mathrm{mag}$ for an age $>0.5 \mathrm{Gyr}$ ). However, optical-NIR colors, such as, e.g., $B-J$ (right panel of Fig. I.17), change by up to $\sim 1.5 \mathrm{mag}$ for ages $>0.5 \mathrm{Gyr}$. Within the above discussed errors, such colors will allow to constrain the age of the stellar LSB host of old $\mathrm{iE} / \mathrm{nE}$ BCDs with a precision of 

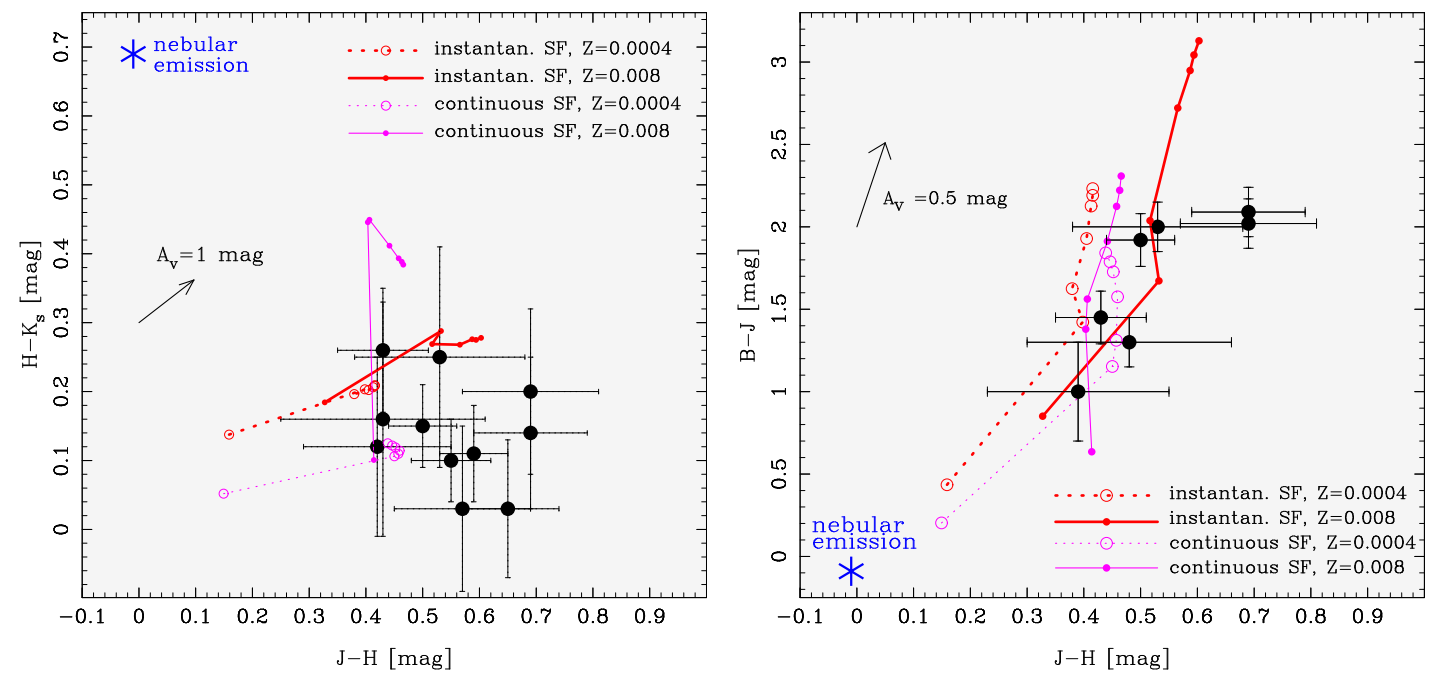

Fig. I.17. Colors of the stellar LSB host galaxies (filled circles) as listed in Tab. I.3. For comparison, we show the color evolution of synthetic stellar populations, calculated with the GALEV evolutionary synthesis model (Schulz et al. 2002; Anders, Bicker \& Fritze-v.Alvensleben 2002, priv. comm.). An initial mass function with a Salpeter slope and respective lower and upper stellar mass cutoffs of 0.08 and $100 \mathrm{M}_{\odot}$ was assumed. We show single-age stellar populations, formed in an instantaneous burst, as well as in a continuous process with a constant SFR, both calculated for metallicities of $Z_{\odot} / 50$ and $Z_{\odot} / 2.5$. For clarity, only population ages of $0.1,0.5$, $1,3,7,10$ and $14 \mathrm{Gyr}$ are shown. The youngest $(0.1 \mathrm{Gyr})$ point of each evolutionary track corresponds to the bluest $H-K_{s}$ and $B-J$ color. All NIR colors shown are transformed to the 2MASS photometric system (see Sect. I.2.4.1), while the $B$ band refers to the Johnson system. Note that the error bars indicate cumulative uncertainties due to all major error sources (see Sect. I.3.5). The colors of ionized gas emission from a metal-deficient $\left(Z_{\odot} / 20\right) \mathrm{H}$ II region $\left(T_{e}=10^{4} \mathrm{~K}, n_{e}=100 \mathrm{~cm}^{-3}\right.$, Case B recombination; cf. Krüger 1992) are indicated by the asterisk. Arrows show reddening vectors.

1-2 Gyr, once precise spectroscopic measurements of its metallicity become available (see the discussion of Gil de Paz et al. 2000b). Fig. I.17 shows that the observed colors are generally compatible with the GALEV predictions within their $1 \sigma$ uncertainties. This is also the case for model predictions from PEGASE (Fioc \& Rocca-Volmerange 1997).

Five BCDs in our sample, Pox4, Pox4B, Tol 1400-411, Tol 65 and Tol 1214-277 (Tab. I.3), show blue $(J-H<0.5, B-J<1.5)$ colors in their LSB component. This may be partly due to an appreciable contribution of extended nebular emission, as suggested by previous optical spectrophotometric work. The blue optical-NIR LSB colors of Tol 65 and Tol 1214-277 may be attributed to a comparatively young ( $\lesssim 1$ Gyr) photometrically dominant stellar population (cf. Papaderos et al P99, F01). The optical-NIR colors of the LSB host of the remaining sample BCDs can be reconciled with an old stellar population of subsolar metallicity. This result is in line with most published optical-NIR photometry on stellar hosts of BCDs (Gil de Paz et al. 2000b, Vanzi et al. 2002, Papaderos et al. 2002), which indicates a comparable range of colors for the LSB population of BCDs. 
We can compare the NIR colors of the LSB component with data from the literature for three sample BCDs, only (Tol 3, Haro 14 and UM 461). For Tol 3, our results compare well with the colors derived by Vanzi et al. (2002) over the whole radius range of $\left(R^{*}=0^{\prime \prime} \ldots 50^{\prime \prime}\right)$. The NIR colors for Tol 3 and Haro 14 agree with those derived by Doublier et al. (2001) for small radii, only (for $R^{*} \lesssim 15^{\prime \prime}$ and $R^{*} \lesssim 10^{\prime \prime}$, respectively). For larger radii, however, the NIR colors by Doublier et al. are not compatible with our results within the $1 \sigma$ uncertainties. Outside the star-forming component the color profiles by Doublier et al. approach values of $J-H>2$ for Tol 3, and $J-H \approx$ $1.0 \cdots>2.5, H-K \approx 0 \cdots-0.6$ for Haro 14 . For UM 461, their color profiles vary between $\approx 1.1 \ldots 2.3(J-H)$ and $\approx-0.6 \ldots 0.5(H-K)$.

\section{I.6 Summary and Conclusions}

We have analyzed deep Near-Infrared (NIR) $J, H, K$ images of 12 Blue Compact Dwarf (BCD) Galaxies and one luminous Blue Compact Galaxy. These objects, together with those studied in an accompanying paper (Cairós et al. C02b), constitute the first part of a sample of 40 BCDs for which deep NIR images were obtained in the framework of a large-scale multi-wavelength study. The limiting surface brightnesses of our data, $\sim 23.5$ to $25.5 \mathrm{mag} / \square^{\prime \prime}$ in $J$ and $\sim 22$ to $24 \mathrm{mag} / \square^{\prime \prime}$ in $K$, allow for the detection and study of the NIR structural properties and colors of the underlying stellar low-surface brightness (LSB) component in all sample galaxies. This evolved LSB host, underlying the star-forming regions is known to exist in the majority of BCDs and to contain the bulk of the stellar mass in these systems. Consequently, a systematic determination of its structural properties (e.g., radial stellar surface density distribution, central surface brightness, exponential scale length) and of the gravitational potential it forms may prove crucial for the understanding of the starburst activity, dynamics and evolution of BCDs. Other than in optical wavelengths, where extended starburst emission hides the LSB host inside its inner 2-3 exponential scale lengths, NIR studies allow to extend surface photometry of the underlying old stellar background to a smaller galactocentric distance, thereby better constrain its overall intensity distribution. Our results can be summarized as follows:

1. Surface brightness profiles (SBPs) of our sample BCDs can at large galactocentric radii $R^{*}$ be well approximated by an exponential fitting law, in agreement with previous observational evidence based on deep optical surface photometry. Also, the exponential scale lengths derived in the optical and NIR spectral domain are in mutual agreement, implying minor optical-NIR color gradients in the LSB component.

2. The LSB host galaxy of several sample BCDs shows on a galactocentric radius comparable to the size of the star-forming component (within its inner 1-3 exponential scale lengths) a conspicuous intensity depression with respect to the purely exponential slope, observed for larger radii. In this regard it is worth mentioning that this type of an inwards flattening exponential SBP, classified type $\mathrm{V}$ in Binggeli \& Cameron (1991), has been frequently derived in dwarf irregular and 
dwarf elliptical galaxies. The rare detection of type $\mathrm{V}$ profiles in $\mathrm{BCD}$ from previous optical studies can be attributed to the fact that in those wavelengths extended starburst emission overshines the underlying LSB population within typically its inner $\sim 2$ exponential scale lengths. A possible high frequency of type $\mathrm{V}$ profiles among dwarf galaxies will not have a notable effect on the structural difference between BCDs and dwarf irregulars on the parameter space defined by the central surface brightness, exponential scale length and absolute magnitude of their LSB host galaxy. However, it may have important implications for our view about BCDs, as it would significantly increase the estimated starburst-to-LSB luminosity fraction, and therefore the amount of photometric fading of these systems, once the starburst activity has terminated. This information is crucial for, e.g., establishing or discarding the hypothesis of faint dwarf spheroidals being the evolutionary endpoints of BCDs. In the same way, a type $\mathrm{V}$ intensity distribution would impose new observational constraints to the derivation of the total stellar mass and its radial density distribution within the underlying LSB host galaxy of BCDs.

3. The exact shape and physical origin of type V SBPs in dwarf galaxies are still to be investigated. We find that such SBPs can be well approximated by a modified exponential fitting formula proposed in Papaderos et al. (1996a). Alternatively, a Sérsic law can also yield good fits to type $\mathrm{V}$ profiles, albeit small systematic residuals. However, the practical applicability of the Sérsic law to the LSB emission of BCDs is limited by the strong non-linear coupling of its free parameters, and the extreme sensitivity of the achieved solutions to, e.g., small uncertainties in the sky subtraction and SBP derivation.

4. For the majority of the LSB host galaxies in our sample we derive optical-NIR colors indicative of an evolved stellar population of subsolar metallicity. The metaldeficient BCDs Tol 65 and Tol 1214-277 show blue colors ( $B-J<1.3$ mag) in their underlying stellar LSB component.

5. NIR images, in combination with optical data reveal for some BCDs signatures of appreciable and non-uniform dust absorption on a spatial scale as large as $\sim 1$ kpc. The NIR images, being less affected by dust extinction and widespread ionized gas emission than optical data, allow for the detection of a variety of morphological features within the star-forming component, as e.g. coherent assemblies of compact stellar clusters, that might be remnants from previous episodes of collective star formation. An elaborate multiwavelength investigation of such sources holds the promise of a better understanding of the history and spatial progression of starforming activity in BCDs.

Acknowledgement. Research by K.G.N. has been supported by the Deutsche Forschungsgemeinschaft (DFG) Grants FR325/50-1 and FR325/50-2. Research by P.P. and K.J.F. has been supported by the Deutsches Zentrum für Luft- und Raumfahrt e.V. (DLR) under grant 50 OR 9907 7. L.M.C. acknowledges support from the European Community Marie Curie Grant HPMF-CT-2000-00774. We thank U. Fritze - v. Alvensleben, P. Anders, J. Bicker and J. Schulz for kindly providing the GALEV models. This research has made use of the NASA/IPAC Extragalactic Database (NED) which is operated by the Jet Propulsion Labo- 
ratory, CALTECH, under contract with the National Aeronautic and Space Administration. This publication makes use of data products from the Two Micron All Sky Survey, which is a joint project of the University of Massachusetts and the Infrared Processing and Analysis Center/California Institute of Technology, funded by the National Aeronautics and Space Administration and the National Science Foundation.

\section{References}

Alton, P. B., Draper, P. W., Gledhill, T. M., Stockdale, D. P., Scarott, S. M. \& Wolstencroft, R. D. 1994, MNRAS, 270, 238

Andredakis, Y. C., Peletier, R. F. \& Balcells, M. 1995, MNRAS, 275, 874

Andreon, S. 2002, A\&A, 382, 821

Babul, A. \& Rees, M. J. 1992, MNRAS, 255, 346

Beck, S. C., Kelly, D. M. \& Lacy, J. H. 1997, AJ, 114, 585

Bergvall, N. \& Östlin, G. 2002, A\&A, 390, 891

Bertelli, G., Bressan, A., Chiosi, C., Fagotto, F. \& Nasi, E. 1994, A\&AS, 106, 275

Binggeli, B. \& Cameron, L. M. 1991, A\&A, 252, 27

Binggeli, B. \& Jerjen, H. 1998, A\&A, 333, 17

Cairós, L. M. 2000, PhD Thesis, Instituto de Astrofísica de Canarias

Cairós, L. M., Caon, N., García-Lorenzo, B., Vílchez, J. M. \& Muñoz-Tuñón, C. 2002a, ApJ, 577, 164

Cairós, L. M., Caon, N., Papaderos, P., Noeske, K. G., Vílchez, J. M., García Lorenzo, B. \& Muñoz-Tuñon, C. 2002b, ApJ, submitted

Cairós, L. M., Caon, N., Vílchez, J. M., González-Pérez, J. \& Muñoz-Tuñón, C. 2001b, ApJS, 136, 393

Cairós, L. M., Vílchez, J. M., González Pérez, J., Iglesias-Páramo, J. \& Caon, N. 2001a, ApJS, 133, 321

Cairós, L. M., et al. 2003, in prep.

Campbell, A. W. \& Terlevich, R. 1984, MNRAS, 211, 15

Cannon, J. M., Skillman, E. D., Garnett, D. R. \& Dufour, R. J. 2002, ApJ, 565, 931

Caon, N., Capaccioli, M. \& D'Onofrio, M. 1993, MNRAS, 265, 1013

Cardelli, J. A., Clayton, G. C. \& Mathis, J. S. 1989, ApJ, 345, 245

Carpenter, J. M. 2001, AJ, 121, 2851

Cellone, S. A. \& Buzzoni, A. 2001, A\&A, 369, 742

Cellone, S. A., Forte, J. C. \& Geisler, D. 1994, ApJS, 93, 397

Conti, P. S. \& Vacca, W. D. 1994, ApJ, 423, L97

Corbin, M. R., Korista, K. T. \& Vacca, W. D. 1993, AJ, 105, 1313

Cutri, R. M., et al. 2000, The 2MASS Explanatory Supplement

Davidge, T.J. 2002, PASP, in press (astro-ph/0208424)

Davies, J. I. \& Phillipps, S. 1988, MNRAS, 233, 553

Dekel, A. \& Silk, J. 1986, ApJ, 303, 39

de Vaucouleurs, G., de Vaucouleurs, A., Corwin, H. G., Buta, R. J., Paturel, G. \& Fouque, P. 1991, Third Reference Catalogue of bright Galaxies (Springer Verlag)

Doublier, V., Caulet, A. \& Comte, G. 1999, A\&AS, 138, 213

Doublier, V., Caulet, A. \& Comte, G. 2001, A\&A, 367, 33

Doublier, V., Comte, G., Petrosian, A., Surace, C. \& Turatto, M. 1997, A\&AS, 124, 405

Duc, P.-A., Papaderos, P., Balkowski, C., Cayatte, V., Thuan, T. X. \& van Driel, W. 1999, A\&AS, 136, 539 
Elias, J. H., Frogel, J. A. \& Humphreys, R. M. 1985, ApJS, 57, 91

Ferrara, A. \& Tolstoy, E. 2000, MNRAS, 313, 291

Fioc, M. \& Rocca-Volmerange, B. 1997, A\&A, 326, 950

Freeman, K. C. 1970, ApJ, 160, 811

Fricke, K. J., Izotov, Y. I., Papaderos, P., Guseva, N. G. \& Thuan, T. X. 2001, AJ, 121, 169

(F01)

Fritz, T. 2000, PhD Thesis, University of Bonn

Fujita, A., Mac Low, M.-M., Meiksin, A. \& Ferrara, A. 2001, American Astronomical Society Meeting, 198, 5703

Gallagher, J. S. III. \& Hunter, D. A. 1989, AJ, 98, 806

García, A. M. 1993, A\&AS, 100, 47

Gil de Paz, A., Zamorano, J., Gallego, J. \& Domínguez, F. de B. 2000a, A\&AS, 145, 377

Gil de Paz, A., Zamorano, J. \& Gallego, J. 2000b, A\&A, 361, 465

González-Riestra, R., Rego, M. \& Zamorano, J. 1987, A\&A, 202, 72

Graham, A., Lauer, T. R., Colless, M. \& Postman, M. 1996, ApJ, 465, 534

Guseva, N. G., Izotov, Y. I., Papaderos, P., Chaffee, F. H., Foltz, C. B., Green, R. F., Thuan, T. X., Fricke, K. J. \& Noeske, K. G. 2001, A\&A, 378, 756

Guseva, N.G., Izotov, Y,I. \& Thuan, T.X. 2000, ApJ, 531, 776

Guseva, N.G., et al. 2002, in prep.

Guzmán, R., Jangren, A., Koo, D. C., Bershady, M. A. \& Simard, L. 1998, ApJ, 495, L13

Hensler, G., Dickow, R. \& Junkes, N. 1997, RMxAA Conf. Series, 6, 90

Heydari-Malayeri, M., Melnick, J. \& Martin, J.-M. 1990, A\&A, 234, 99

Hidalgo-Gámez, A. M., Masegosa, J. \& Olofsson, K. 2001, A\&A, 367, 388

Hidalgo-Gámez, A. M., Masegosa, J. \& Olofsson, K. 2002, RMxAA Conf. Series, 12, 242

Hirashita, H., Tamura, N. \& Takeuchi, T. T. 2000, IAU Symposium, 201, 64

Hunt, L.K., Thuan,T.X., Izotov, Y.I. 2003, ApJ, in press

Hunter, D. \& Hoffman, L. 1999, AJ, 117, 2789

Izotov Y. I., Chaffee, F. H. \& Green, R. F. 2001, ApJ, 562, 727

Izotov, Y. I. \& Thuan, T. X. 1999, ApJ, 511, 639

Izotov, Y. I. \& Thuan, T. X. 2002, ApJ, 567, 875

Izotov, Y. I., Thuan, T. X. \& Lipovetsky, V. A. 1997, ApJS, 108, 1

James, P. A. 1994, MNRAS, 269, 176

Jarrett, T. H., Chester, T., Cutri, R., Schneider, S. \& Huchra, J. 2000, AJ, 119, 2498

Johnson, K. E., Leitherer, C., Vacca, W. D. \& Conti, P. S. 2000, AJ, 120, 1273

Karachentsev, I. D., Sharina, M. E., Dolphin, A. E., et al. 2002, A\&A, 385, 21

Kobulnicky, H. A., Kennicutt, R. C. \& Pizagno, J. L. 1999, ApJ, 514, 544

Krüger, H. 1992, PhD Thesis, University of Göttingen

Krüger, H., Fritze-v. Alvensleben, U. \& Loose, H.-H. 1995, A\&A, 303, 41

Kunth, D., Maurogordato, S. \& Vigroux, L. 1988, A\&A, 204, 10

Kunth, D. \& Sargent, W. L. W. 1981, A\&A, 101, L5

Kunth, D. \& Sargent, W. L. W. 1983, ApJ, 273, 81

Legrand, F. 2000, A\&A, 354, 504

Lin, D. N. C. \& Faber, S. M. 1983, ApJ, 266, L21

Loose, H. H. \& Thuan, T. X. 1986, Star Forming Dwarf Galaxies and Related Objects, 73 (LT86)

Lowenthal, J. D., Koo, D. C., Guzman, R., et al. 1997, ApJ, 481, 673

MacArthur, L. A., Courteau, S. \& Holtzman, J. A. 2002, ApJ, in press (astro-ph/0208404)

Makarova, L. N., Karachentsev, I. D., Grebel, E. K. \& Barsunova, O. Y. 2002, A\&A, 384, 72 
Makarova, L., Karachentsev, I., Takalo, L. O., Heinaemaeki, P. \& Valtonen, M. 1998, A\&AS, 128, 459

Marlowe, A. T., Heckman, T. M., Wyse, R. F. G. \& Schommer, R. 1995, ApJ, 438, 563

Marlowe, A. T., Meurer, G. R. \& Heckman, T. M. 1999, ApJ, 522, 183

Marlowe, A. T., Meurer, G. R., Heckman, T. M. \& Schommer, R. 1997, ApJS, 112, 285

Masegosa, J., Moles, M. \& Campos-Aguilar, A. 1994, ApJ, 420, 576

Mas-Hesse, J. M. \& Kunth, D. 1999, A\&A, 349, 765

Méndez, D.I. \& Esteban, C. 1999, AJ, 118, 2723

Méndez, D.I. \& Esteban, C. 2000, A\&A, 359, 493

Meurer, G. R., Staveley-Smith, L. \& Killeen, N. E. B. 1998, MNRAS, 300, 705

Mirabel, I. F. \& Sanders, D. B. 1988, ApJ, 335, 104

Noeske, K. G. 1999, Diploma Thesis, University of Göttingen

Noeske, K. G., Guseva, N. G., Fricke, K. J., Izotov, Y. I., Papaderos, P. \& Thuan, T. X. 2000, A\&A, 361, 31 (Paper IV)

Noeske, K. G., Iglesias-Páramo, J., Vílchez, J. M., Papaderos, P. \& Fricke, K. J. 2001, A\&A, 371, 806 (Paper VI)

Noguchi, M. 2001, ApJ, 555, 289

Ott, J., Walter, F., Brinks, E., Van Dyk, S. D., Dirsch, B. \& Klein, U. 2001, AJ, 122, 3070

Pagel, B. E. J., Simonson, E. A., Terlevich, R. J. \& Edmunds, M. G. 1992, MNRAS, 255, 325

Papaderos, P., et al. 2003, in prep.

Papaderos, P. 1998, PhD Thesis, University of Göttingen

Papaderos, P. \& Fricke, K. J 1998, Highlights in X-ray Astronomy, B. Aschenbach et al. (eds.)

Papaderos, P., Fricke, K. J., Thuan, T. X., Izotov, Y. I. \& Nicklas, H. 1999, A\&A, 352, L57 (P99)

Papaderos, P., Izotov, Y.I., Thuan, T.X., Noeske, K.G., Guseva, N.G. \& Fricke, K.J. 2002, A\&A, 393, 461

Papaderos, P., Loose, H.-H., Fricke, K. J. \& Thuan, T. X. 1996b, A\&A, 314, 59 (P96b)

Papaderos, P., Loose, H.-H., Thuan, T.X. \& Fricke, K.J. 1996a, A\&AS, 120, 207 (P96a)

Pastoriza, M. G. \& Dottori, H. A. 1981, ApJ, 244, 27

Patterson, R. J. \& Thuan, T. X. 1996, ApJS, 107, 103

Peimbert, M. \& Torres-Peimbert, S. 1974, ApJ, 193, 327

Persson, S. E., Murphy, D. C., Krzeminski, W., Roth, M. \& Rieke, M. J. 1998, AJ, 116, 2475

Pustilnik, S. A., Kniazev, A. Y., Lipovetsky, V. A. \& Ugryumov, A. V. 2001, A\&A, 373, 24

Rieschick, A. \& Hensler, G. 2001, ApSS Suppl., 277, 111

Sage, L. J., Salzer, J. J., Loose, H.-H. \& Henkel, C. 1992, A\&A, 265, 19

Salzer, J. J., MacAlpine, G. M. \& Boroson, T. A. 1989, ApJS, 70, 479

Salzer, J. J. \& Norton, S. A. 1999, ASP Conf. Ser. 170: The Low Surface Brightness Universe, 253

Schaerer, D., Contini, T. \& Kunth, D. 1999, A\&A, 341, 399

Schlegel, D. J., Finkbeiner, D. P. \& Davis, M. 1998, ApJ, 500, 525

Schulte-Ladbeck, R. E., Hopp, U., Greggio, L. \& Crone, M. M. 2000, AJ, 120, 1713

Schulte-Ladbeck, R. E., Hopp, U., Greggio, L., Crone, M. M. \& Drozdovsky, I. O. 2001, AJ, 121, 3007

Schulz, J., Fritze - v. Alvensleben, U., Möller, C. S. \& Fricke, K. J. 2002, A\&A, 392, 1 Sérsic, J.-L. 1968, Atlas de Galaxias Australes, Observatorio Astronomico de Cordoba Silk, J., Wyse, R. F. G. \& Shields, G. A. 1987, ApJ, 322, L59 
Simpson, C. E. \& Gottesman, S. T. 2000, AJ, 120, 2975

Smoker, J. V., Davies, R. D., Axon, D. J. \& Hummel, E. 2000, A\&A, 361, 19

Stasinska, G., Comte, G. \& Vigroux, L. 1986, A\&A, 154, 352

Tammann, G. A. \& Sandage, A. 1985, ApJ, 294, 81

Taylor, C. L., Brinks, E., Grashuis, R. M. \& Skillman, E. D. 1995, ApJS, 99, 427

Telles, E. \& Terlevich, R. 1995, MNRAS, 275, 1

Telles, E., Melnick, J. \& Terlevich, R. 1997, MNRAS, 288, 78

Thuan, T. X. 1991, Massive Stars in Starbursts, C. Leitherer et al. (eds.)

Thuan, T. X. 1985, ApJ, 299, 881

Thuan, T. X., Izotov, Y. I. \& Foltz, C. B. 1999, ApJ, 525, 105

Tully, R. B. 1988, Nearby Galaxies Catalog (Cambridge University Press)

Vacca W.D. \& Conti, P.S. 1992, ApJ, 401, 543

Vallenari, A. \& Bomans, D. J. 1996, A\&A, 313, 713

van den Bergh, S. 2000, AJ, 119, 609

van Zee, L. 2000, AJ, 119, 2757

van Zee, L., Skillman, E.D. \& Salzer, J.J. 1998, AJ, 116, 1186

van Zee, L., Salzer, J.J. \& Skillman, E.D. 2001, AJ, 122, 121

Vanzi, L., Hunt, L. K. \& Thuan, T. X. 2002, A\&A, 390, 481

Vanzi, L., Hunt, L. K., Thuan, T. X. \& Izotov, Y. I. 2000, A\&A, 363, 493

Vanzi, L., Rieke, G. H., Martin, C. L. \& Shields, J. C. 1996, ApJ, 466, 150

Vennik, J., Hopp, U., \& Popescu, C. C. 2000, A\&AS, 142, 399

Vigroux, L., Souviron, J., Lachieze-Rey, M., \& Vader, J. P. 1988, A\&AS, 73, 1

Wainscoat, R. J. \& Cowie, L. L., 1992, AJ, 103, 332

Walter, F., Brinks, E., Duric, N., \& Klein, U. 1997, AJ, 113, 2031

Yasuda, N., Fukugita, M. \& Okamura, S. 1997, ApJS, 108, 417

Young, C. K. \& Currie, M. J. 1994, MNRAS, 268, L11

Young, C. K. \& Currie, M. J. 1995, MNRAS, 273, 1141 


\title{
New insights to the photometric structure of Blue Compact Dwarf Galaxies from deep Near-Infrared studies $^{0}$
}

\section{The second sample of northern BCDs}

\author{
K.G. Noeske, P. Papaderos, L.M. Cairós, K.J. Fricke \\ Universitäts-Sternwarte, Geismarlandstraße 11, D-37083 Göttingen, Germany
}

Summary. This paper is part of a series of publications which describe a systematic study of Blue Compact Dwarf (BCD) Galaxies in the Near Infrared (NIR). Compared to the visible light, NIR data allow for a better separation of the starburst emission from the light distribution of the old stellar low-surface brightness (LSB) host galaxy. We analyze deep NIR broad band images of a sample of $11 \mathrm{BCDs}$, observed with the Calar Alto ${ }^{1} 3.6 \mathrm{~m}$ telescope. This work enlarges the samples presented in preceding papers of this study (Noeske et al. 2003a, Cairós et al. 2003a) by BCDs of the most common morphological type, displaying a regular elliptical LSB host galaxy. The data presented here allow for the detection and quantitative study of the extended stellar LSB host galaxy in all sample BCDs. NIR surface brightness profiles (SBPs) of the LSB host galaxies agree at large galactocentric radii with those from optical studies, showing also an exponential intensity decrease and compatible scale lengths. Similar to Noeske et al. (2003a), we find centrally flattening exponential (type V) SBPs of the host galaxy for several BCDs. Such SBPs remain mostly undetected in optical bands, due to the comparably stronger starburst emission at these wavelengths. We apply a modified exponential distribution (Papaderos et al. 1996a) to decompose and quantitatively analyze SBPs with a type V intensity distribution of the LSB host. We present results of the surface photometry and the decomposition of SBPs, and discuss individual objects with respect to morphological details of their star-forming regions.

\section{II.1 Introduction}

This paper is part of a series of publications, which describe a deep Near Infrared (NIR) imaging and surface photometry study of a large sample of Blue Compact Dwarf (BCD) Galaxies (Noeske et al. 2003a, hereafter N03a, Cairós et al. 2003a, hereafter C03a). As described in these papers, this project aims at an improved understanding of the structure and photometric properties of the old stellar low-surface brightness (LSB) host galaxy, and of the young stellar populations in BCDs. The former component contains practically all stellar mass of typical BCDs, and may therefore be dynamically important (see references in N03a). The radial light distribution of the

\footnotetext{
${ }^{0}$ To be submitted to Astronomy \& Astrophysics.

${ }^{1}$ German-Spanish Astronomical Center, Calar Alto, operated by the Max-Planck-Institute for Astronomy, Heidelberg, jointly with the Spanish National Commission for Astronomy.
} 
LSB host, i.e. its projected luminosity density distribution, provides close constraints to the stellar mass distribution in a BCD. This information is important to model the global gravitational potential and dynamics of BCDs, as well as the effects of starburst events in such objects, such as galactic winds (e.g. De Young \& Heckman 1994, Mac Low \& Ferrara 1999, Silich \& Tenorio-Tagle 2001). The structural parameters (e.g., exponential scale length and central surface brightness) of the stellar LSB host form also a prime diagnostic tool for assessing the proposed evolutionary relations between different types of dwarf galaxies (see N03a and references therein). Of equal interest are the relations between the properties of the stellar host galaxy and the occurrence and properties of starburst activity in BCDs (Papaderos et al. 1996b, hereafter P96b). The investigation of all latter issues requires a separation of the light distributions of the young and old stellar populations by means of a decomposition of radial surface brightness profiles (SBPs). Such analyses are however hampered by the extended dominant starburst emission at visible wavelengths, and can be performed with a much better precision in the NIR (see N03a, C03a).

In the current paper, we present $J, H$ and $K$ image data and surface photometry of a further subsample of BCDs, as well as a decomposition of the derived SBPs into the radial intensity distributions of the old LSB host galaxy and the starburst component. In the same way as in N03a, morphological information is provided for each object, in particular for the star-forming (SF) regions. Remarkable features are briefly discussed. All data reduction and analysis procedures that have been described in N03a were unalteredly applied to the present data set.

The BCDs studied in this paper are meant to extend the samples analyzed in N03a and C03a. Most of the objects in the present sample show an iE/nE morphology, according to the classification scheme by Loose \& Thuan (1986, hereafter LT86). Such BCDs display star forming-regions either irregularly distributed (iE) or centrally confined (nE) within a regular, elliptical or circular stellar LSB host galaxy. Such iE/nE BCDs contribute the majority ( $~ 80-90 \%$ ) of the BCDs in the local Universe (LT86), but were underrepresented in the previous samples studied by N03a and C03a with respect to irregular morphological types and interacting objects. Previous studies have indicated that the various morphological types of BCDs may systematically differ from each other by several physical properties (e.g. Salzer et al. 1989b, Telles et al. 1997, Noeske et al. 2000). For later comparisons of their NIR properties, a balanced representation of different morphological types is therefore desirable. Analyses of the cumulative sample will be presented in forthcoming papers of the present series.

This paper is structured as follows: in Sect. II.2, we list the the subsample under study, and summarize the observations. In Sect. II.3, some details concerning the derivation, analysis and decomposition of SBPs, described in detail in N03a, are repeated to facilitate the understanding of this paper. This section also lists the results of the surface photometry, and of the decomposition of the surface brightness profiles, as well as colors of the old stellar host galaxies. Individual objects are presented and briefly discussed in Sect. II.4, along with images, surface brightness profiles and color profiles. 


\section{II.2 Observations and data reduction}

The galaxies of this sample are listed in Table II.1, along with their adopted distances, absolute $B$ magnitudes, Galactic extinction, and references to literature sources from which the latter values were taken. Selection criteria for distance determinations from the literature, and details on the Galactic extinction values we adopt, are given in Sections 2.1 and 2.4.2 of N03a, respectively.

The NIR images were observed with the $3.6 \mathrm{~m}$ telescope of the German-Spanish Astronomical Center, Calar Alto, Spain. Atmospheric conditions during the different observing runs were generally satisfactory (April 3rd, 1999: FWHM 1".5, transparency average; December 26th, 1999: FWHM 1".3 - 3".5, transparency fair; May 10th-15th, 2000: FWHM 0.8 - 1".4, transparency good to average; October 6th-10th, 2000: FWHM 1".2 - 2".0, transparency good to fair). The telescope was equipped with the OMEGA PRIME camera, mounted at the prime focus. The $1024 \times 1024$ pixel Rockwell HAWAII detector of this instrument provided a pixel scale of 0".396 and a field of view of 6.76. Images were taken through the broad band filters $J$ and $H$, as well as the $K^{\prime}$ filter, which was preferred to a normal $K$ filter to extenuate the contribution of thermal background. Information on the observing technique and control of the time-dependent NIR background emission can be found in N03a. The total on-object exposure times for each galaxy, after rejection of subexposures that were affected by unstable readout electronics or strong background gradients, are listed in columns 3 5 of Table II.1. All data reduction procedures that were applied to the NIR images are detailed in N03a. The angular resolution of the resulting images is listed in column 9 of Table II.1.

\section{II.2.1 Flux calibration and extinction correction}

The transparency variations within each night, $\gtrsim 0.15$ mag in the $K$ band, did not allow for a precise flux calibration through observations of standard stars. All data were therefore calibrated using fluxes of bright (typically $10 \ldots 15 \mathrm{~J}$ mag) field stars in the vicinity of the sample galaxies, given in the Two Micron All Sky Survey (2MASS) catalogue $^{2}$ (see Cutri et al. 2000, Jarrett et al. 2000). To calibrate the $K^{\prime}$ images, the 2MASS $K_{s}$ fluxes were first transformed to $K^{\prime}$ magnitudes using Equations (3) from N03a. The results listed within this paper therefore refer to the $J, H, K^{\prime}$ photometric system defined by the Calar Alto 3.6m telescope and the OMEGA PRIME camera, tied to 2MASS zero points. Since color terms of the transformation between either photometric system are not available, intrinsic uncertainties of few 0.01 mag may be present. Notes on these uncertainties and on comparisons to data calibrated in other photometric systems are given in Sect. 2.4.1 of N03a.

All magnitudes and colors given in this paper are corrected for Galactic extinction as described in Sect. 2.4.2 of N03a, adopting the $B$ band extinction values given in Table II.1.

\footnotetext{
2 http://www.ipac.caltech.edu/2mass/
} 
Table II.1. Sample galaxies

\begin{tabular}{|c|c|c|c|c|c|c|c|c|c|}
\hline 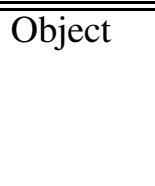 & $\begin{array}{l}\text { RA(J2000) } \\
\text { DEC(J2000) } \\
\text { (2) }\end{array}$ & $\begin{array}{l}\mathrm{t}_{J} \\
{[\mathrm{~s}]} \\
(3)\end{array}$ & $\begin{array}{l}\mathrm{t}_{H} \\
\\
{[\mathrm{~s}]} \\
(4)\end{array}$ & $\begin{array}{l}\mathrm{t}_{K^{\prime}} \\
\\
{[\mathrm{s}]} \\
(5)\end{array}$ & $\begin{array}{c}\mathrm{M}_{B} \\
\text { (ref.) } \\
{[\mathrm{mag}]} \\
(6)\end{array}$ & $\begin{array}{c}\mathrm{A}_{B} \\
\\
{[\mathrm{mag}]} \\
(7)\end{array}$ & $\begin{array}{c}\mathrm{D} \\
\text { (ref.) } \\
{[\mathrm{Mpc}]} \\
(8)\end{array}$ & $\begin{array}{c}\text { FWHM } \\
\text { (final) } \\
{\left[{ }^{\prime \prime}\right.} \\
(9)\end{array}$ & $\begin{array}{l}\begin{array}{l}\text { other } \\
\text { names }\end{array} \\
(10)\end{array}$ \\
\hline $\begin{array}{l}\text { Mkn } 314 \\
\text { (iE) }\end{array}$ & $\begin{array}{r}23^{\mathrm{h}} 02^{\mathrm{m}} 59^{\mathrm{s}} .3 \\
+16^{\circ} 36^{\prime} 18^{\prime \prime} .9 \\
\end{array}$ & 1440 & 1740 & 1920 & $\begin{array}{l}-18.4 \\
\text { (f) }\end{array}$ & 0.37 & $\begin{array}{l}28.9 \\
\text { (a) }\end{array}$ & 1.5 & $\begin{array}{l}\text { NGC 7468; } \\
\text { UGC } 12329\end{array}$ \\
\hline $\begin{array}{l}\text { Mkn } 209 \\
\text { (iE) }\end{array}$ & $\begin{array}{r}12^{\mathrm{h}} 26^{\mathrm{m}} 16^{\mathrm{s}} .0 \\
+48^{\circ} 29^{\prime} 36^{\prime \prime} .6 \\
\end{array}$ & 1620 & $\overline{1440}$ & $\overline{1140}$ & $\begin{array}{c}-14.2 \\
(\mathrm{~g}) \\
\end{array}$ & 0.07 & $\begin{array}{l}5.8 \\
\text { (b) } \\
\end{array}$ & 1.5 & $\begin{array}{l}\text { UGCA 281; IZw 36; } \\
\text { Haro } 29\end{array}$ \\
\hline $\begin{array}{l}\begin{array}{l}\text { Mkn } 996 \\
(\mathrm{nE})\end{array} \\
\end{array}$ & $\begin{array}{r}01^{\mathrm{h}} 27^{\mathrm{m}} 35^{\mathrm{s}} .5 \\
-06^{\circ} 19^{\prime} 36^{\prime \prime} .1 \\
\end{array}$ & 1260 & 1440 & $\overline{1200}$ & $\begin{array}{c}-16.6 \\
(\mathrm{~h})\end{array}$ & 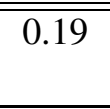 & $\begin{array}{c}20.4 \\
(\mathrm{c})\end{array}$ & 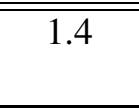 & \\
\hline $\begin{array}{l}\text { Mkn 370 } \\
(\mathrm{nE})\end{array}$ & $\begin{array}{r}02^{\mathrm{h}} 40^{\mathrm{m}} 29^{\mathrm{s}} .0 \\
+19^{\circ} 17^{\prime} 50^{\prime \prime} .1\end{array}$ & 11140 & 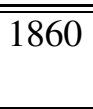 & $\overline{1640}$ & $\begin{array}{c}-16.9 \\
\text { (f) }\end{array}$ & $\overline{0.40}$ & $\begin{array}{c}11.2 \\
\text { (a) }\end{array}$ & $\overline{1.2}$ & $\begin{array}{l}\text { NGC 1036; IC 1828; } \\
\text { UGC } 2160\end{array}$ \\
\hline $\begin{array}{l}\text { I Zw } 115 \\
\text { (iI) }\end{array}$ & $\begin{array}{r}15^{\mathrm{h}} 32^{\mathrm{m}} 57^{\mathrm{s}} .0 \\
+46^{\circ} 27^{\prime} 06^{\prime \prime} .5 \\
\end{array}$ & 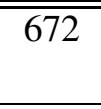 & 672 & 480 & $\begin{array}{c}-16.4 \\
(\mathrm{~g}) \\
\end{array}$ & $\overline{0.40}$ & $\begin{array}{c}15.0 \\
\text { (a) }\end{array}$ & $\overline{1.3}$ & $\begin{array}{l}\text { UGC 9893; } \\
\text { VV 720 }\end{array}$ \\
\hline $\begin{array}{l}\text { Mkn 5 } \\
\text { (iE/iI,C) }\end{array}$ & $\begin{array}{r}06^{\mathrm{h}} 42^{\mathrm{m}} 15^{\mathrm{s}} .5 \\
+75^{\circ} 37^{\prime} 32^{\prime \prime} .6 \\
\end{array}$ & 1650 & 2100 & $\overline{\overline{2340}}$ & $\begin{array}{l}-15.7 \\
\text { (f) }\end{array}$ & $\overline{0.36}$ & $\begin{array}{c}15.3 \\
\text { (a) }\end{array}$ & 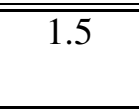 & " UGCA 130 \\
\hline
\end{tabular}


Table II.1. (continued)

\begin{tabular}{|c|c|c|c|c|c|c|c|c|c|}
\hline OFject & $\begin{array}{l}\text { RA(J2000) } \\
\text { DEC(J2000) } \\
\text { (2) }\end{array}$ & $\begin{array}{l}\mathrm{t}_{J} \\
{[\mathrm{~s}]} \\
(3)\end{array}$ & $\begin{array}{l}\mathrm{t}_{H} \\
{[\mathrm{~s}]} \\
(4)\end{array}$ & $\begin{array}{l}\mathrm{t}_{K^{\prime}} \\
\\
{[\mathrm{s}]} \\
(5)\end{array}$ & $\begin{array}{c}\mathrm{M}_{B} \\
\text { (ref.) } \\
{[\mathrm{mag}]} \\
(6)\end{array}$ & $\begin{array}{c}\mathrm{A}_{B} \\
{[\mathrm{mag}]} \\
(7)\end{array}$ & $\begin{array}{c}\text { D } \\
\text { (ref.) } \\
{[\mathrm{Mpc}]} \\
(8)\end{array}$ & $\begin{array}{c}\text { FWHM } \\
\text { (final) } \\
{\left[{ }^{\prime \prime}\right]} \\
(9)\end{array}$ & $\begin{array}{l}\begin{array}{l}\text { other } \\
\text { names }\end{array} \\
(10)\end{array}$ \\
\hline $\begin{array}{l}\text { Mkn } 600 \\
\text { (iE) }\end{array}$ & $\begin{array}{r}02^{\mathrm{h}} 51^{\mathrm{m}} 04^{\mathrm{s}} \cdot 6 \\
+04^{\circ} 27^{\prime} 13^{\prime \prime} .9\end{array}$ & 1560 & 1320 & 1080 & $\begin{array}{c}-15.4 \\
\text { (f) }\end{array}$ & 0.28 & $\begin{array}{l}12.6 \\
\text { (a) }\end{array}$ & 1.1 & \\
\hline $\begin{array}{l}\text { NGC } 6789 \\
\text { (iE) }\end{array}$ & $\begin{array}{r}19^{\mathrm{h}} 16^{\mathrm{m}} 41^{\mathrm{s}} .9 \\
+63^{\circ} 58^{\prime} 18^{\prime \prime} .0 \\
\end{array}$ & "1200 & "1680 & "1920 & $\begin{array}{l}-14.3 \\
\text { (i) }\end{array}$ & 0.30 & $\begin{array}{l}3.6 \\
\text { (d) }\end{array}$ & 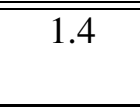 & 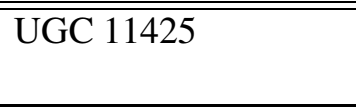 \\
\hline $\begin{array}{l}\text { Mkn } 324 \\
\text { (iE) }\end{array}$ & $\begin{array}{r}23^{\mathrm{h}} 26^{\mathrm{m}} 32^{\mathrm{s}} .8 \\
+18^{\circ} 15^{\prime} 59^{\prime \prime} .0\end{array}$ & 1680 & 600 & 1140 & $\begin{array}{c}-16.4 \\
\text { (f) }\end{array}$ & 0.22 & $\begin{array}{c}21.8 \\
\text { (a) }\end{array}$ & 1.4 & UGCA 439 \\
\hline $\begin{array}{l}\text { Mkn } 450 \\
\text { (iE) }\end{array}$ & $\begin{array}{r}13^{\mathrm{h}} 14^{\mathrm{m}} 48^{\mathrm{s}} .3 \\
+34^{\circ} 52^{\prime} 51^{\prime \prime} .3 \\
\end{array}$ & 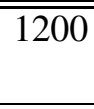 & 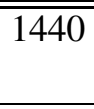 & 1920 & $\begin{array}{c}-16.7 \\
(\mathrm{j}) \\
\end{array}$ & 0.06 & $\begin{array}{c}17.9 \\
(\mathrm{a})\end{array}$ & 1.3 & $\begin{array}{l}\text { UGC 08323; VV 616; } \\
\text { HS 1312+3508 }\end{array}$ \\
\hline $\begin{array}{l}\text { NGC } 5058 \\
\text { (iI?) }\end{array}$ & $\begin{array}{r}13^{\mathrm{h}} 16^{\mathrm{m}} 52^{\mathrm{s}} .3 \\
+12^{\circ} 32^{\prime} 55^{\prime \prime} .3\end{array}$ & 720 & 1680 & 1440 & $\begin{array}{l}-15.9 \\
\text { (i) }\end{array}$ & 0.13 & $\begin{array}{l}11.6 \\
(\mathrm{c}, \mathrm{e})\end{array}$ & 1.1 & $\begin{array}{l}\text { UGC 08345; Mkn 786; } \\
\text { KPG } 370\end{array}$ \\
\hline
\end{tabular}

A: Resolution of the best image set available for the respective galaxy, after reduction and combination (a): Tully (1988), (b): Schulte-Ladbeck et al. 2001, (c): inferred from the heliocentric velocity $v_{\text {hel }}$ listed in the NED, corrected for solar motion with respect to the center of the Virgo Cluster and adopting $\mathrm{H}_{0}=75 \mathrm{~km} \mathrm{~s}^{-1} \mathrm{Mpc}^{-1}$ (cf. N03a), (d): Drozdovsky et al. (2001), (e): see Sect. II.4.11, (f): $\mathrm{m}_{B}$ from Cairós et al. C01a, (g): $\mathrm{m}_{B}$ from Papaderos et al. 1996a, (h): $\mathrm{m}_{B}$ from Thuan et al. 1996, (i): $\mathrm{m}_{B}$ from the RC3 (de Vaucouleurs et al. 1991), (j): $\mathrm{m}_{B}$ from Vennik et al. 2000. 


\section{II.3 Surface photometry and profile decomposition}

The SBPs and color profiles were derived as detailed in N03a, using throughout the algorithm "iv" presented in Papaderos et al. 2002. A comparison to SBPs derived by alternative methods, mostly "i" and "iii" described in Papaderos et al. 1996a (hereafter P96a), was performed for each profile to ensure consistency at both low and high $S / N$ levels.

Also the decomposition of the derived SBPs into the intensity distributions of the LSB host and the starburst component, required to separately study the emission of either stellar component, is explained in Sect. 3.2 of N03a.

For a better understanding of the results of the present work (Table II.2), we reiterate here the functions that were employed to fit the SBPs of the LSB host galaxies. The resulting parameters of those fits provide the structural parameters of the LSB host, and allow to calculate a model of its intensity distribution, which is then subtracted from the total SBPs to obtain the light distribution of the starburst component.

At large galactocentric radii $(>2 \ldots 3$ scale lengths $\alpha)$, the SPBs of the LSB hosts show for most BCDs a smooth decay, which can typically be approximated by the exponential law (e.g. LT86, P96a, Telles et al. 1997, Cairós et al. 2001a, hereafter C01a).

If the intensity is expressed in terms of the surface brightness $\mu$, this function reads as

$$
\mu\left(R^{\star}\right)=\mu_{\mathrm{E}, 0}+1.086 \frac{R^{\star}}{\alpha}
$$

with $\mu_{\mathrm{E}, 0}, \alpha$ and $R^{\star}$ denoting, respectively, the extrapolated central surface brightness in mag/ $\square^{\prime \prime}$, the exponential scale length, and the photometric radius.

As detailed in N03a, an exponential fit to the outer SBP of the LSB host provided in some cases no meaningful decomposition, but exceeded the intensity of the observed total SBP at small radii. In these cases, where a central flattening of the outer exponential intensity distribution of the LSB host ("type V SBP", Binggeli \& Cameron 1991, see Sect. 3.2 in N03a) had to be postulated, a modified exponential distribution Eq. (II.2) was applied (cf. P96a) to decompose the total SBPs:

$$
I\left(R^{\star}\right)=I_{\mathrm{E}, 0} \exp \left(-\frac{R^{\star}}{\alpha}\right)\left\{1-q \exp \left(-P_{3}\left(R^{\star}\right)\right)\right\}
$$

with

$$
P_{3}\left(R^{\star}\right)=\left(\frac{R^{\star}}{b \alpha}\right)^{3}+\left(\frac{R^{\star}}{\alpha} \cdot \frac{1-q}{q}\right)
$$

This empirical function, in the following referred to as med, flattens with respect to a pure exponential law inside of a cutoff radius $b \alpha$, and attains at $R^{*}=0^{\prime \prime}$ an intensity given by the relative depression parameter $q=\Delta I / I_{\mathrm{E}, 0}<1$. Details on the determination of the parameters of the med, $\mu_{\mathrm{E}, 0}, \alpha$, and $(b, q)$, can be found in Sect. 3.2 of N03a. 
For several objects, the SBPs of the LSB host show small systematic deviations from an exponential slope, yet at low significance levels. Those BCDs were therefore decomposed by means of exponential fits. Nevertheless, a differing LSB profile shape is possible for Mkn 370, and type V SBPs appear likely for Mkn 209, Mkn 314, Mkn 600 and NGC 5058.

Alternative fits to the light distribution of the LSB host, using the Sérsic law instead of the med, were abandoned in the present work. As was extensively discussed in N03a and C03a, the use of the latter empirical function to model the LSB emission of $\mathrm{BCDs}$ in connected to principal problems.

Sérsic fits were merely used to describe the total SBPs. In the case of BCDs, the latter include the emission of both, the starburst component and the LSB host galaxy, which differ strongly from each other with respect to their mass-to-light ratios. These Sérsic fits are therefore of purely formal nature, to allow comparisons to previous surface photometry studies which performed such fits.

The photometric quantities that were derived for the sample BCDs are summarized in Table II.2. BCDs without significant signatures of a type $\mathrm{V}$ profile in their underlying LSB component, decomposed by means of a pure exponential (Eq. II.1), are marked with an asterisk in column 1. For the remaining systems we list the $(b, q)$ parameters, as obtained respectively by fitting Eq. (II.2) to SBPs of the LSB host galaxy. The latter were derived from images out of which irregular starburst emission had been largely removed (N03a). Columns 3 and 4 list, respectively, the extrapolated central surface brightness $\mu_{\mathrm{E}, 0}$ and exponential scale length $\alpha$, obtained by fitting an exponential (Eq. II.1) to the outer exponential LSB part of each SBP. Column 5 lists the total apparent magnitude of the LSB component, computed by extrapolating the fitted model (i.e. either an exponential or a med) to $R^{\star}=\infty$. Columns 6 through 9 list the radii and magnitudes of the star-forming $(\mathrm{P})$ and underlying stellar LSB component (E), as obtained by profile decomposition. Following P96a, we measure the respective radial extent $\left(P_{\text {iso }}, E_{\text {iso }}\right)$ and encircled magnitude $\left(m_{P_{\text {iso }}}, m_{E_{\text {iso }}}\right)$ of each component at an isophotal level $i s o$, taken to be $23 \mathrm{mag} / \square^{\prime \prime}$ for $J$ and $22 \mathrm{mag} / \square^{\prime \prime}$ for $H$ and $K$. The isophotal radii determined for the sample BCDs at $23 J$ mag/ $\square^{\prime \prime}$ turn out to be comparable to those obtained from optical SBPs at $25 B \mathrm{mag} / \square^{\prime \prime}$ ( $P_{25}$ and $E_{25}$ in P96a). Column 10 lists the magnitude from an SBP integration out to the last data point, and total magnitudes from aperture measurements (cf. Sect. II.3.2) are listed in column 11. The radii $r_{\text {eff }}$ and $r_{80}$, enclosing $50 \%$ and $80 \%$ of the SBP's flux are included in column 12 . Finally, a formal Sérsic exponent for the whole SBP $\left(\eta_{\mathrm{SBP}}\right)$, for later comparison with literature data, is listed in column 13 of Tab. II.2.

\section{II.3.1 Colors of the underlying LSB host galaxy}

Colors of the LSB host galaxy were derived as the error-weighted mean of the color profiles, outside radii affected by SF activity (cf. Sect. 3.5. in N03a). Deviant points, being probably affected by uncertainties in the sky determination, and local residuals from the subtraction of background sources were rejected. The mean colors of the host galaxies are shown at the right edge of each color profile (Figs. II.1 - II.11), and are 
Table II.2. Structural properties of the $\operatorname{dwarfs}^{a}$; see also the discussion of individual objects

\begin{tabular}{|c|c|c|c|c|c|c|c|c|c|c|c|c|}
\hline $\begin{array}{l}\text { Name } \\
(b, q)^{b} \\
\eta_{\mathrm{LSB}}^{c} \\
(1)\end{array}$ & Band & $\begin{array}{c}\mu_{E, 0} \\
\mathrm{mag} / \square^{\prime \prime}\end{array}$ & $\begin{array}{c}\alpha \\
\mathrm{kpc}\end{array}$ & $\begin{array}{c}m_{\mathrm{LSB}}^{\mathrm{fit}} \\
\text { mag }\end{array}$ & $\begin{array}{l}P_{\text {iso }} \\
\text { kpc }\end{array}$ & $\begin{array}{c}m_{P_{\text {iso }}} \\
\text { mag }\end{array}$ & $\begin{array}{l}E_{\text {iso }} \\
\mathrm{kpc}\end{array}$ & $\begin{array}{c}m_{\mathrm{E}_{\mathrm{iso}}} \\
\mathrm{mag}\end{array}$ & $\begin{array}{c}m_{\mathrm{SBP}} \\
\mathrm{mag}\end{array}$ & $\begin{array}{c}m_{\text {tot }} \\
\text { mag }\end{array}$ & $\begin{array}{c}r_{\mathrm{eff}}, r_{80} \\
\mathrm{kpc}\end{array}$ & $\eta_{\mathrm{SBP}}$ \\
\hline Mkn 314 & $J$ & $19.10 \pm 0.13$ & $1.00 \pm 0.04$ & 12.84 & 1.85 & 12.84 & 3.58 & 12.99 & 12.12 & 12.05 & $0.88,1.86$ & 1.46 \\
\hline \multirow[t]{2}{*}{$\star^{f}$} & $H$ & $18.61 \pm 0.15$ & $1.09 \pm 0.05$ & 12.17 & 1.70 & 12.26 & 3.39 & 12.39 & 11.51 & 11.38 & $0.90,1.96$ & 1.50 \\
\hline & $K^{\prime}$ & $18.73 \pm 0.17$ & $1.22 \pm 0.07$ & 12.04 & 2.12 & 11.77 & 3.67 & 12.28 & 11.21 & 11.17 & $0.88,1.88$ & 1.45 \\
\hline Mkn 209 & $J$ & $20.21 \pm 0.06$ & $0.23 \pm 0.01$ & 13.63 & 0.16 & 16.37 & 0.60 & 13.98 & 13.69 & 13.49 & $0.29,0.47$ & 1.69 \\
\hline \multirow[t]{2}{*}{$\star^{f}$} & $H^{d}$ & $19.08 \pm 0.31$ & $0.18 \pm 0.02$ & 13.08 & 0.02 & 18.83 & 0.48 & 13.39 & 13.38 & 13.16 & $0.29,0.45$ & 1.31 \\
\hline & $K^{\prime}$ & - & - & - & - & - & - & - & 13.10 & & $0.28,0.44$ & 1.37 \\
\hline Mkn 996 & $J$ & $18.20 \pm 0.03$ & $0.40 \pm 0.01$ & 13.17 & 0.67 & 14.50 & 1.77 & 13.24 & 12.91 & 12.87 & $0.51,1.01$ & 1.53 \\
\hline \multirow[t]{2}{*}{$\star$} & $H$ & $17.80 \pm 0.04$ & $0.42 \pm 0.01$ & 12.65 & 0.69 & 13.74 & 1.64 & 12.76 & 12.33 & 12.26 & $0.51,1.03$ & 1.67 \\
\hline & $K_{e}^{\prime}$ & $17.48 \pm 0.05$ & $0.43 \pm 0.01$ & 12.32 & 0.68 & 13.20 & 1.77 & 12.41 & 12.04 & 11.94 & $0.42,0.84$ & 1.90 \\
\hline Mkn 370 & $J$ & $18.85 \pm 0.05$ & $0.64 \pm 0.01$ & 11.52 & 0.80 & 12.80 & 2.43 & 11.64 & 11.29 & 11.27 & $0.72,1.49$ & 1.76 \\
\hline \multirow[t]{2}{*}{$\star^{g}$} & $H$ & $18.36 \pm 0.06$ & $0.63 \pm 0.01$ & 11.04 & 0.84 & 12.14 & 2.12 & 11.22 & 10.74 & 10.72 & $0.71,1.46$ & 1.72 \\
\hline & $K^{\prime}$ & $18.00 \pm 0.22$ & $0.59 \pm 0.04$ & 10.83 & 0.78 & 12.03 & 2.17 & 10.97 & 10.57 & 10.46 & $0.67,1.39$ & 1.55 \\
\hline IZw 115 & $J$ & $19.52 \pm 0.13$ & $0.53 \pm 0.02$ & 13.68 & 0.85 & 15.05 & 1.47 & 14.15 & 13.46 & 13.26 & $0.88,1.46$ & 1.15 \\
\hline \multirow[t]{2}{*}{$(2.4,0.8)$} & $H$ & $19.23 \pm 0.35$ & $0.56 \pm 0.06$ & 13.25 & 0.80 & 14.49 & 1.18 & 14.10 & 13.00 & 12.89 & $0.88,1.46$ & 1.15 \\
\hline & $K^{\prime}$ & - & - & - & - & - & - & - & 12.87 & 12.64 & $0.80,1.32$ & 1.13 \\
\hline \multirow{3}{*}{$\begin{array}{l}\text { Mkn 5 } \\
(1.4,0.65)\end{array}$} & $J$ & $19.03 \pm 0.04$ & $0.37 \pm 0.01$ & 13.73 & 0.54 & 15.90 & 1.35 & 13.90 & 13.73 & 13.58 & $0.56,0.92$ & 1.08 \\
\hline & $H$ & $18.78 \pm 0.07$ & $0.40 \pm 0.01$ & 13.30 & 0.58 & 14.94 & 1.18 & 13.56 & 13.21 & 13.16 & $0.57,0.92$ & 1.04 \\
\hline & $K^{\prime}$ & $18.41 \pm 0.06$ & $0.40 \pm 0.01$ & 12.92 & 0.56 & 14.86 & 1.33 & 13.14 & 12.93 & 12.78 & $0.57,0.91$ & 1.22 \\
\hline
\end{tabular}


Table II.2. (continued)

\begin{tabular}{|c|c|c|c|c|c|c|c|c|c|c|c|c|}
\hline $\begin{array}{l}\text { Name } \\
(b, q)^{b} \\
\eta_{\mathrm{LSB}}^{c} \\
(1)\end{array}$ & Band & $\begin{array}{c}\mu_{E, 0} \\
\mathrm{mag} / \square^{\prime \prime}\end{array}$ & $\begin{array}{c}\alpha \\
\mathrm{kpc}\end{array}$ & $\begin{array}{c}m_{\mathrm{LSB}}^{\mathrm{fit}} \\
\text { mag }\end{array}$ & $\begin{array}{l}P_{\text {iso }} \\
\mathrm{kpc}\end{array}$ & $\begin{array}{c}m_{P_{\text {iso }}} \\
\text { mag }\end{array}$ & $\begin{array}{l}E_{\text {iso }} \\
\mathrm{kpc}\end{array}$ & $\begin{array}{c}m_{\mathrm{E}_{\text {iso }}} \\
\text { mag }\end{array}$ & $\begin{array}{c}m_{\mathrm{SBP}} \\
\text { mag }\end{array}$ & $\begin{array}{c}m_{\text {tot }} \\
\text { mag }\end{array}$ & $\begin{array}{c}r_{\mathrm{eff}}, r_{80} \\
\mathrm{kpc}\end{array}$ & $\eta_{\mathrm{SBP}}$ \\
\hline Mkn 600 & $J$ & $19.35 \pm 0.08$ & $0.30 \pm 0.01$ & 13.93 & 0.41 & 15.63 & 0.99 & 14.11 & 13.86 & 13.78 & $0.35,0.64$ & 1.34 \\
\hline \multirow[t]{2}{*}{$\star^{f}$} & $H$ & $19.09 \pm 0.08$ & $0.32 \pm 0.01$ & 13.48 & 0.40 & 15.07 & 0.87 & 13.79 & 13.41 & 13.23 & $0.36,0.66$ & 1.35 \\
\hline & $K_{d}^{\prime}$ & $19.01 \pm 0.25$ & $0.32 \pm 0.04$ & 13.42 & 0.43 & 14.67 & 0.88 & 13.14 & 13.25 & 13.13 & $0.35,0.65$ & 1.52 \\
\hline NGC 6789 & $J$ & $18.79 \pm 0.13$ & $0.20 \pm 0.01$ & 11.93 & 0.31 & 13.96 & 0.75 & 12.11 & 11.82 & 11.78 & $0.33,0.57$ & 1.07 \\
\hline \multirow[t]{2}{*}{$(3.3,0.70)$} & $H$ & $18.37 \pm 0.08$ & $0.20 \pm 0.01$ & 11.51 & 0.25 & 13.56 & 0.64 & 11.78 & 11.38 & 11.32 & $0.33,0.56$ & 1.13 \\
\hline & $K^{\prime}$ & $18.12 \pm 0.31$ & $0.20 \pm 0.02$ & 11.25 & 0.23 & 13.58 & 0.69 & 11.48 & 11.19 & 11.08 & $0.34,0.57$ & 1.26 \\
\hline Mkn 324 & $J$ & $17.81 \pm 0.03$ & $0.28 \pm 0.01$ & 13.68 & 0.57 & 14.61 & 1.35 & 13.73 & 13.32 & 13.25 & $0.34,0.67$ & 1.26 \\
\hline \multirow[t]{2}{*}{$\star$} & $H$ & $17.36 \pm 0.10$ & $0.29 \pm 0.01$ & 13.17 & 0.55 & 14.08 & 1.24 & 13.26 & 12.81 & 12.75 & $0.34,0.68$ & 1.30 \\
\hline & $K_{d}^{\prime}$ & $16.93 \pm 0.12$ & $0.26 \pm 0.01$ & 12.94 & 0.50 & 14.07 & 1.24 & 13.00 & 12.66 & 12.55 & $0.32,0.62$ & 1.23 \\
\hline Mkn 450 & $J$ & $19.91 \pm 0.04$ & $0.87 \pm 0.01$ & 13.09 & 1.41 & 14.41 & 2.48 & 13.43 & 12.90 & 12.80 & $1.25,2.08$ & 1.46 \\
\hline \multirow[t]{2}{*}{$(1.5,0.65)$} & $H^{d}$ & $19.17 \pm 0.13$ & $0.80 \pm 0.03$ & 12.55 & 1.18 & 14.18 & 2.08 & 12.96 & 12.37 & 12.35 & $1.22,2.01$ & 1.06 \\
\hline & $K^{\prime}$ & 一 & 一 & - & - & 一 & 一 & 一 & 12.16 & 12.05 & $1.20,1.99$ & 1.27 \\
\hline \multirow{3}{*}{$\begin{array}{l}\text { NGC } 5058 \\
{ }_{\star}^{f}\end{array}$} & $J$ & $19.08 \pm 0.13$ & $0.47 \pm 0.02$ & 12.47 & 0.72 & 13.67 & 1.70 & 12.62 & 12.21 & 12.18 & $0.56,1.13$ & 1.44 \\
\hline & $H$ & $18.95 \pm 0.05$ & $0.52 \pm 0.01$ & 12.14 & 0.80 & 12.71 & 1.45 & 12.42 & 11.65 & 11.67 & $0.55,1.10$ & 1.42 \\
\hline & $K^{\prime}$ & $18.62 \pm 0.14$ & $0.49 \pm 0.02$ & 11.94 & 0.79 & 12.56 & 1.52 & 12.15 & 11.49 & 11.48 & $0.52,1.02$ & 1.46 \\
\hline
\end{tabular}

a: All values are corrected for Galactic extinction, adopting the $A_{B}$ from Tab. II.1 (cf. Sect. II.2.1).

$b, c$ : See Sect. II.3 for details. Objects for which the LSB component was modelled by a pure exponential (Eq. II.1) are marked with an asterisk

$d$ : Decomposition less reliable (faint object, or data affected by nearby bright stars).

$e: K^{\prime}$ SBP less deep than $J$ and $H$; effects on columns (11)-(13).

$f$ : small systematic deviations from an exponential LSB profile; type V SBP appears likely (see Sect. II.3).

$g$ : small systematic deviations from an exponential LSB profile (see Sect. II.3). 
listed in Table II.3. The $H-K^{\prime}$ colors given in this table were transformed to $H-K_{s}$ to facilitate comparisons to other photometric systems.

Table II.3. Colors of the host galaxy ${ }^{a}$

\begin{tabular}{lll}
\hline Object & $\begin{array}{l}J-H \\
{[\mathrm{mag}]}\end{array}$ & $\begin{array}{l}H-K_{s} \\
{[\mathrm{mag}]}\end{array}$ \\
\hline Mkn 314 & $0.77 \pm 0.17^{b}$ & $0.20 \pm 0.12^{b}$ \\
\hline Mkn 209 & $0.37 \pm 0.13^{b}$ & $0.19 \pm 0.18^{b}$ \\
\hline Mkn 996 & $0.56 \pm 0.12$ & $0.31 \pm 0.13^{b}$ \\
\hline Mkn 370 & $0.52 \pm 0.14$ & $0.16 \pm 0.17$ \\
\hline IZw 115 & $0.46 \pm 0.15^{b}$ & $0.26 \pm 0.26^{b}$ \\
\hline Mkn 5 & $0.54 \pm 0.15^{b}$ & $0.45 \pm 0.12^{b}$ \\
\hline Mkn 600 & $0.50 \pm 0.17^{b, c}$ & $0.08 \pm 0.29^{b, c}$ \\
\hline NGC 6789 & $0.42 \pm 0.12$ & $0.31 \pm 0.18^{b}$ \\
\hline Mkn 324 & $0.55 \pm 0.12$ & $0.22 \pm 0.18^{b}$ \\
\hline Mkn 450 & $0.51 \pm 0.16$ & $0.19 \pm 0.25^{b}$ \\
\hline NGC 5058 & $0.41 \pm 0.16$ & $0.24 \pm 0.25^{b}$ \\
\hline
\end{tabular}

${ }^{a}$ : Corrected for galactic extinction (see Sect. II.2.1);

${ }^{b}$ : possible local instabilities in one SBP at low $S / N$ levels.

${ }^{c}$ : possible contamination by nebular emission over a large portion of the LSB host galaxy

Colors are given in the NIR photometric system described in Sect. II.2.1; the $H-K_{s}$ colors were calculated from the $H-K^{\prime}$ colors using transformations given in N03a. Errors include cumulative uncertainties in the determination of color profiles, and of the calibration of the Calar Alto data by means of 2MASS zero points (see Sect. II.2.1).

\section{II.3.2 Unsharp masking technique \& aperture photometry}

To improve on studies of fine morphological details, the hierarchical binning (hb) transformation, a modified unsharp masking technique described in e.g. Papaderos (1998), was applied to the data. Features of interest are displayed in the insets of Figures II.1 - II.11.

Total magnitudes of the galaxies were derived within polygonal apertures which extend typically out to 1.5 Holmberg radii (column 11 of Table II.2), after removal of fore- and background sources from the area of interest (cf. Sect. II.2). These magnitudes can generally be considered more accurate than those inferred from an integration of SBPs (see N03a).

\section{II.4 Results and discussion of individual objects}

\section{II.4.1 Mkn 314 (NGC 7468, UGC 12329)}

As an iE-classified system, Mkn 314 represents the most common morphological BCD type (LT86). Optical surface photometry as well as deep $\mathrm{H} \alpha$ imaging have been pre- 

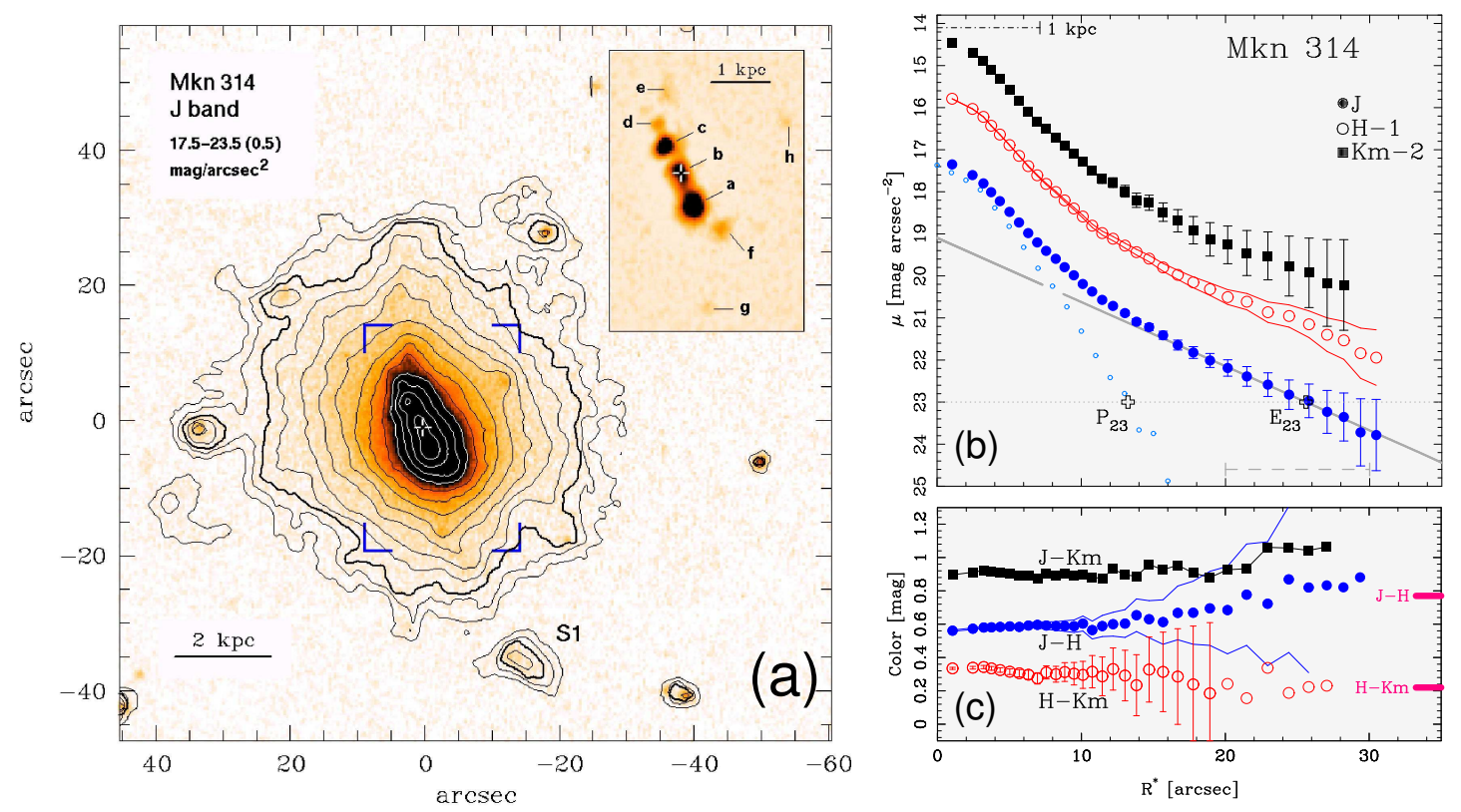

Fig. II.1. a) Contours overlaid with a $J$ image of Mkn 314 ( $D=28.9 \mathrm{Mpc})$. Contours, corrected for Galactic extinction, go from 17.5 to $23.5 \mathrm{~J} \mathrm{mag} / \square^{\prime \prime}$ in steps of $0.5 \mathrm{mag}$. The $23 \mathrm{~J} \mathrm{mag} / \square^{\prime \prime}$ isophote is depicted by the thick contour. The inset shows a contrast-enhanced (see Sect. II.3.2) and magnified version of the central region of the BCD (indicated by brackets in the contour map image). Compact sources arranged in a bar-like structure along the major axis of the BCD are marked, with the labeling of sources a-c following the denomination by Mazzarella \& Boroson (1993). The bright star-forming region $b$ is marked with a white cross in both the main plot and the inset. The detached SW source S1 shows active star formation. b) Surface brightness profiles (SBPs) of Mkn 314 in the $J, H$ and $K_{s}$, corrected for galactic extinction. For a better visualization, the $H$ and $K_{s}$ SBPs are shifted by -1 and -2 mag, respectively. The thick solid line shows an exponential fit to the stellar LSB component in $J$ (cf. Sect. II.3), computed within the radius range indicated by the long-dashed bar at the bottom of the diagram. The emission in excess to the fit (small open circles) is attributable to the starburst component, which dominates the light in the inner part of Mkn 314. The isophotal radii $P_{23}$ and $E_{23}$ of the star-forming and LSB component at the surface brightness level of $23 \mathrm{~J}$ mag/ $\square^{\prime \prime}$ (horizontal dotted line) are indicated. The bar at the upper left indicates a galactocentric radius of $1 \mathrm{kpc}$. c) Color profiles, computed by subtraction of the SBPs (panel $\mathbf{b}$ ). The thick lines at the rightmost part of the diagram indicate the mean $J-H$ and $H-K^{\prime}$ colors of the LSB component (see Sect. II.3.1).

sented in C01ab. This intrinsically luminous BCD $\left(\mathrm{M}_{B}=-18.4\right.$; Cairós et al. 2001b, hereafter $\mathrm{C} 01 \mathrm{~b}$ ) was morphologically selected as a candidate polar-ring galaxy (Whitmore et al. 1990, van Driel et al. 2001), and was included in the study of Markarian galaxies with multiple nuclei by Mazzarella \& Boroson (1993). Optical broad-band images by the latter authors revealed three prominent maxima, roughly arranged along the major axis of the galaxy (labeled a through $\mathrm{c}$ in Figure II.1a). Nordgren et al. (1995) found that regions $a$ and $c$, situated at comparable projected distances $\left(\approx 10^{\prime \prime} 5\right.$ or 0.57 kpc and 9.4 or $0.52 \mathrm{kpc}$, respectively) from the optically brightest, central source b, show velocity differences to the latter of $30 \pm 17$ and $20 \pm 14 \mathrm{~km} \mathrm{~s}^{-1}$. These values are 
of the order of typical H I velocity dispersions in BCDs (van Zee et al. 1998), and do not provide any strong evidence for kinematical distortions which might indicate a previous merger event. Narrow band images reveal that SF activity in the galaxy is distributed along the northeast-southwest direction, in a bar-like structure, which extends to about $5 \mathrm{kpc}$ southwest from the nuclear region (Deeg et al. 1997, C01b). BCDs showing such bar-structures are relatively frequent (for instance Mkn 370, this paper; Mkn 35, II Zw 71 and II Zw 33, C01b) and are morphologically classified as "chain starburst" in $\mathrm{C} 01 \mathrm{~b}$. The total $\mathrm{H} \alpha$ flux of the galaxy amounts to $\sim 5.2 \times 10^{-13} \mathrm{erg} \mathrm{cm}^{-2}$ $\mathrm{s}^{-1}$. In the $\mathrm{H} \alpha$ frames, three strong SF regions are detected, aligned with the central sources detected in the broad-band frames. The peak of the $\mathrm{H} \alpha$ emission is located at knot $c$, whereas that $b$ and $a$ are moderate sources.

The morphologies in the optical and in the NIR basically coincide, though the NIR frames provide a better spatial resolution. Smaller condensations, surrounding the latter three major sources, were identified (named $d-h$ in Fig. II.1a ).

The starburst population is immersed within an extended older population of stars, which displays elliptical isophotes and red colors $(B-R \approx 1$, Cairós et al. 2001a, hereafter C01a; C01b). Since the chain of SF sources along the major axis of Mkn 314 is less prominent in the NIR than in optical wavelengths (cf. C01b), slight differences in the outer slope of optical and NIR SBPs of the LSB component are to be expected. In $J$ and $H$ we derive a scale length of $\sim 1 \mathrm{kpc}$, somewhat smaller than the optical value $(\sim 1.2 \mathrm{kpc}, \mathrm{C} 01 \mathrm{a})$. NIR SBPs reveal a slight curvature for $R^{*} \geq 20^{\prime \prime}$ pointing to a type V SBP; however, the low $S / N$ level in this outermost region does not permit to corroborate this conclusion.

A tail structure, formed by several SF knots, departs from the central regions into the south-west direction, connecting with an extended source named "S1" in Fig. II.1a . This object, which could be detected in all NIR bands, splits into two compact H II regions on $\mathrm{H} \alpha$ frames $(\mathrm{C} 01 \mathrm{~b})$. The blue optical colors ( $B-R \sim 0.5 \mathrm{mag}$; $\mathrm{C} 01 \mathrm{~b}$ ) of $\mathrm{S} 1$ are comparable to those observed in the central part of the BCD (regions a through $\mathrm{C}$, $\mathrm{C} 01 \mathrm{~b})$. From the present data we cannot judge whether $\mathrm{S} 1$ is a gaseous or stellar interloper infalling onto Mkn 314: the projected velocity difference of $30 \mathrm{~km} \mathrm{~s}^{-1}$ (Nordgren et al. 1995) to Mkn 314 is not unusual for close dwarf companions of BCDs (Noeske et al. 2001a). Also, Taylor et al. (1994) find from interferometric H I observations of Mkn 314 little indications for an interaction and describe merely an "oval distortion" of the gaseous component.

\section{II.4.2 Mkn 209 (UGCA 281, I Zw 36, Haro 29)}

Mkn 209 provides an example of an intrinsically faint $\left(\mathrm{M}_{B}=-14.2\right.$, Schulte-Ladbeck et al. 2001), compact (optical radius of $\approx 0.6 \mathrm{kpc}$ at $25 B \mathrm{mag} / \square^{\prime \prime}$, P96a) and metaldeficient $\left(\mathrm{Z} \approx Z_{\odot} / 14\right.$, Izotov \& Thuan 1999) BCD with an iE-morphology (LT86). This galaxy has been the subject of numerous studies, due to its relative proximity ( $D \approx$ 3.6 Mpc, Schulte-Ladbeck et al. 2001).

The SF regions of Mkn 209 are morphologically reminiscent of those in Mkn 5 (Sect. II.4.6), and are dominated by two bright sources (a and b, Fig. II.2a). Gil de 

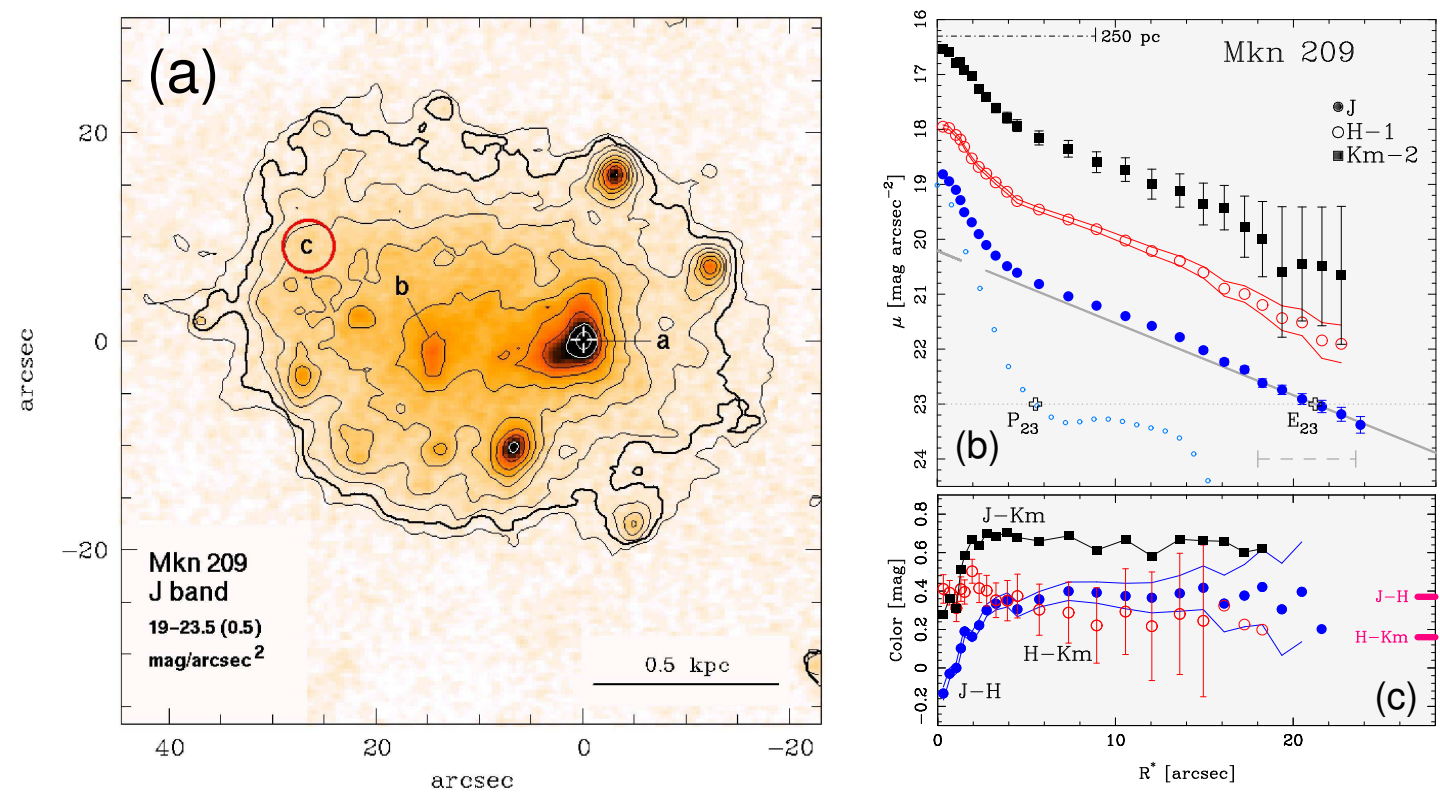

Fig. II.2. Mkn 209 ( $D=5.8$ Mpc). For explanations of symbols and labels, refer to Fig. II.1. a) $J$ band image and isophotes. The $\mathrm{H} \alpha$-emitting SF regions a and b, as well as the blue region c, almost absent in NIR images, are marked. b),c) Surface brightness and color profiles.

Paz et al. (2003, hereafter GMP03) found that the $\mathrm{H} \alpha$ emission peaks at the western $\mathrm{SF}$ region a (Fig. II.2a). Additional diffuse $\mathrm{H} \alpha$ emission is also present further to the east, close to region $\mathrm{b}\left(\approx 18^{\prime \prime}\right.$ from $\left.\mathrm{a}\right)$. HST observations of knot a (Deharveng et al. 1994) showed the young stars to have ages $\leq$ than 12 Myr, in agreement with spectral evolutionary synthesis models by Mas-Hesse \& Kunth (1999), who derived a burst age of $\approx 2.7$ Myr. Spectroscopic studies of Mkn 209 furthermore revealed the presence of Wolf-Rayet stars (Schaerer et al. 1999, Guseva et al. 2000).

The source $c$ (Fig. II.2a), detected in optical images $\sim 15^{\prime \prime}$ northeast of of $b$, is nearly absent in the NIR. As indicated by its blue colors on uncalibrated optical-NIR color maps, and the presence of a local $\mathrm{H} \alpha$ maximum (GMP03), region $\mathrm{C}$ may be dominated by nebular emission. The faintness of this source in the NIR may indicate that the stellar population in this area is still too young ( $\lesssim 8 \mathrm{Myr}$ ) to be dominated by Red Super Giant (RSG) stars.

From an IUE UV spectral study, Fanelli et al. (1988) conjectured that Mkn 209 could be a young galaxy, currently undergoing its first episode of star formation. A later HST study by Deharveng et al. (1994) showed, however, that the observed red colors could not be attributed to RSG stars alone, but required the presence of an older stellar population. An old population was confirmed by P96a, who reported almost constant, red ( $B-R \sim 1$ ) colors for the extended, elliptical stellar host galaxy of Mkn 209, first detected by LT86. Recent $J$ and $H$ color magnitude diagram studies of Mkn 209, using HST NICMOS data (Schulte-Ladbeck et al. 2001), showed the presence of stars with ages $>1-2 \mathrm{Gyr}$, in agreement with the above results. 
The SBPs of the LSB host can only be analyzed outside the very extended plateau emission of the starburst, for $R^{*}>18^{\prime \prime}$. An exponential fit, when extrapolated inwards, does nowhere imply an intensity brighter than the observed one. It leads, however, to a local depression of the derived radial intensity distribution of the starburst component at $R^{*} \sim 7^{\prime \prime}$. A type V LSB profile, fitted by a med (Eq. II.2) with a cutoff radius $b \alpha \approx 14^{\prime \prime}$, could provide a more plausible decomposition, but cannot be confirmed, due to the numerous fore- and background sources which limit the reliability of the SBPs at their outermost data points $\left(R^{*} \gtrsim 20^{\prime \prime}\right)$.

\section{II.4.3 Mkn 996}
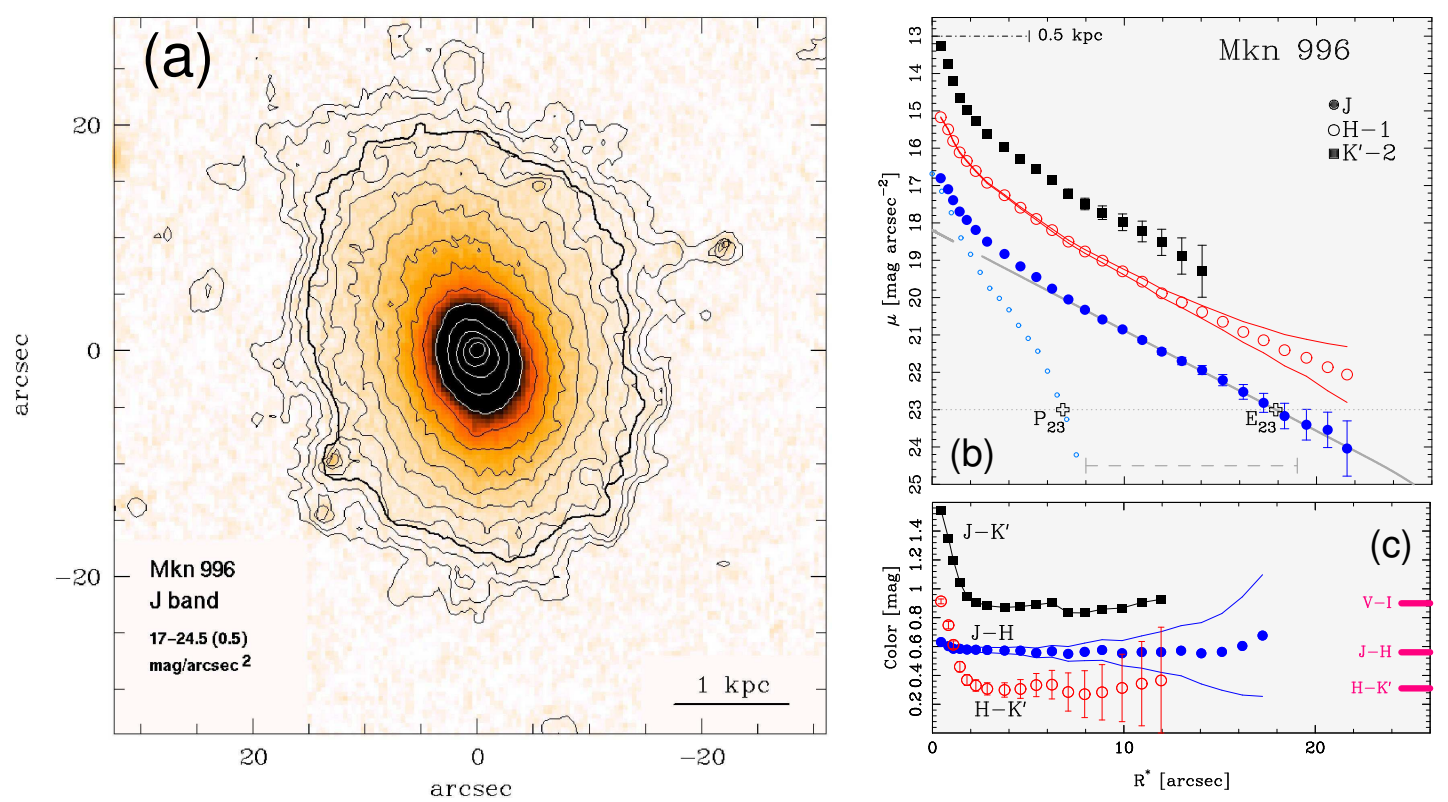

Fig. II.3. Mkn 996 ( $D=20.4$ Mpc). For explanations of symbols and labels, see Fig. II.1. a) $J$ band image and isophotes. b),c) Surface brightness and color profiles. The indicated $V-I$ color of the LSB host galaxy has been adopted from Thuan et al. (1996).

Mkn $996\left(M_{B}=-16.6 \mathrm{mag}\right)$ may be considered a prototypical example of the $\mathrm{nE}$ BCD class. It shows a low oxygen abundance $\left(Z \approx Z_{\odot} / 10\right.$; Thuan et al. 1996), typical for BCDs, and a very compact morphology of its starburst component, situated near the geometrical center of its smooth LSB host galaxy. Optical spectra revealed strong Wolf-Rayet features, characteristic of WN and WC stars (Thuan et al. 1996, Schaerer et al. 1999) and imply an unusually high electron density of $\sim 5 \times 10^{4} \mathrm{~cm}^{-3}$ (Thuan et al. 1996). HST WFPC2 $V$ and $I$ data (Thuan et al. 1996) showed SF activity to be confined to the inner $\approx 3^{\prime \prime}\left(R^{*} \approx 315 \mathrm{pc}\right)$ of the BCD. The same data revealed dust patches which extend to the north of the central SF region, as well as a central two-arm spiral pattern with a size of $160 \mathrm{pc}$. The presence of considerable amounts of dust is also indicated by the NIR color profiles (Fig. II.3c), displaying unusually red colors within the central $2^{\prime \prime}$ which cannot be accounted for by stellar or nebular emission (cf. 
e.g. Figures 1 or 17 in N03a). Measurements in apertures, several times larger than the angular resolution of the images, confirm that this color excess is not attributable to possible resolution effects in the color profiles.

Another intriguing property of this galaxy is a strongly asymmetric distribution of several globular clusters with an age of $\approx 10 \mathrm{Gyr}$, in the very outskirts of the LSB component.

Surface photometry studies by Thuan et al. (1996) showed that for $R^{*} \gtrsim 8^{\prime \prime}$ the stellar LSB host can be well fitted by an exponential law with a scale length $\alpha=420 \mathrm{pc}$. The colors of the outer regions of the galaxy $(V-I \approx 0.9)$ are characteristic of an old LSB population. These results are in good agreement with our NIR surface photometry, from which we infer an exponential intensity decrease of the LSB component with a scale length between 400 and $430 \mathrm{pc}$. Both our results, and those by Thuan et al. (1996) are in contrast with the conclusion by Doublier et al. (1999), that Mkn 996 follows an overall de Vaucouleurs-profile. This disagreement might stem from a subtle excess for $R^{*}>18^{\prime \prime}$, visible in both our SBPs and those by Thuan et al. (1996), which at first glance points to a shallow, more extended LSB population. We believe, however, that this small excess originates from the combined emission of the asymmetrically distributed globular cluster population, discovered by Thuan et al. (1996). These sources cannot be properly resolved and subtracted using ground-based data, and could therefore induce a slight flattening of the SBPs at large radii. We therefore fitted the SBPs for $R^{*}<18^{\prime \prime}$ in $J$ only, i.e. at radii where the emission can be reliably attributed to the unresolved stellar LSB population of Mkn 996.

\section{II.4.4 Mkn 370 (NGC 1036, UGC 02160)}

With respect to other BCDs, the iE-classified (LT86) BCD Mkn 370 is particularly metal-rich $\left(\mathrm{Z} \approx Z_{\odot} / 2, \mathrm{M}_{B}=-16.9 ; \mathrm{C} 01 \mathrm{~b}\right)$. Deep broad-band surface photometry in the optical and high-resolution color and $\mathrm{H} \alpha$ maps have been first presented in C01a,b. A dedicated spectrophotometric study of this BCD has recently been published in Cairós et al. (2002).

Two major sources, separated by $\approx 7^{\prime \prime}(380 \mathrm{pc})$, are located in the central region of the galaxy. These correspond to the "double nucleus" catalogued in Mazzarella \& Boroson (1993) and are labeled $a$ and b in Figure II.4a, following the nomenclature of the latter authors. As apparent from the contrast-enhanced inset in the $J$ band image (Fig. II.4a), the elongated central region a splits into a brighter southwestern and a fainter northeastern source, similar to what was reported by Nordgren et al. (1995). The NIR images show several fainter sources, roughly aligned with the major axis of region a.

The positions of both $a$ and $b$ coincide in the optical and in the NIR, although $b$, which strongly emits in the blue bands, is only weakly detected in the NIR. The blue colors of this source $(U-B=-0.79)$, together with the flat emission line spectrum and the large $\mathrm{H} \alpha$ equivalent width $(E W(\mathrm{H} \alpha) \approx 500 \AA$; Nordgren et al. 1995, Cairós et al.2002) are indicative of a young stellar population (ages $\leq 5 \mathrm{Myr}$ ) and substantial ionized gas contribution. Knot a presents slightly redder colors $(U-B=-0.60)$ and a 

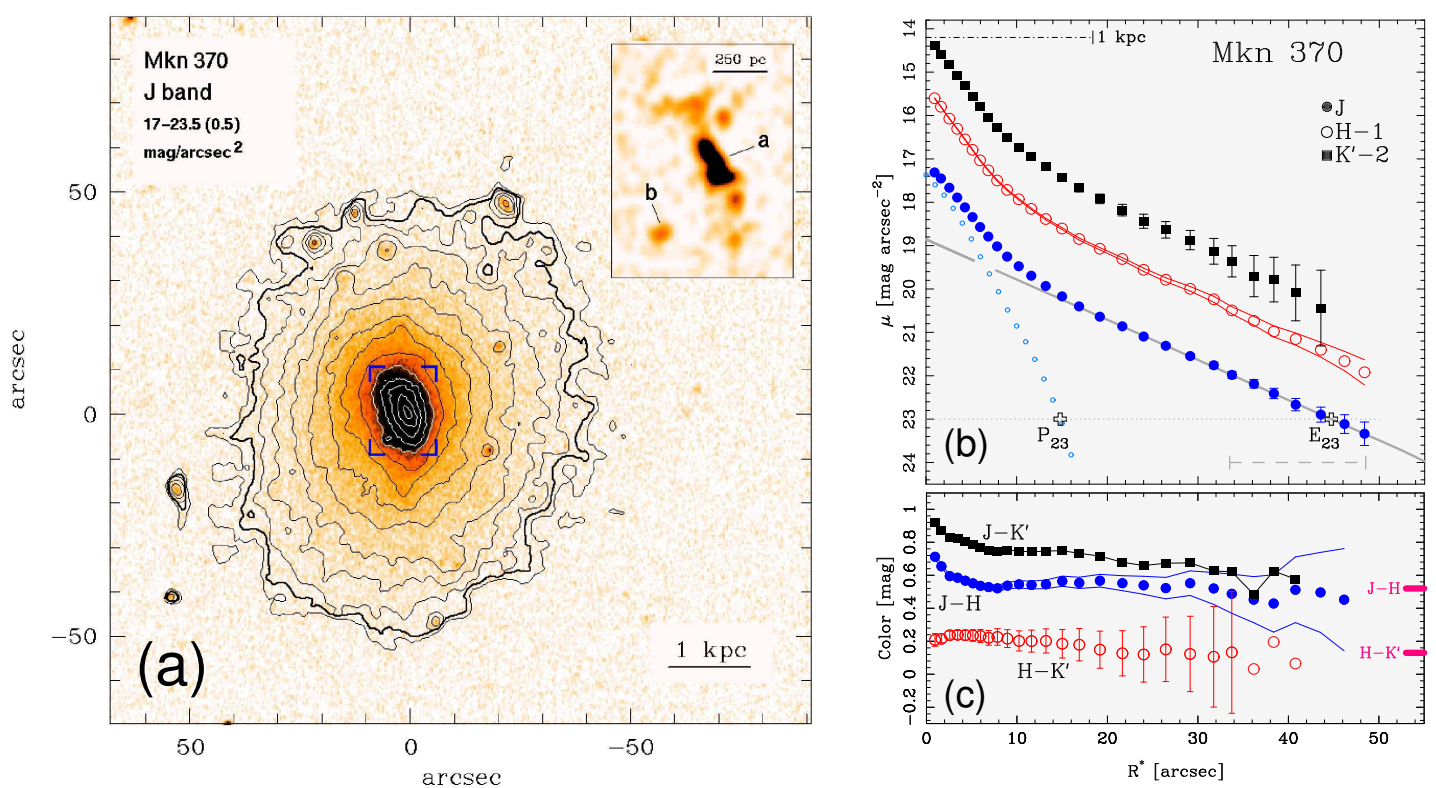

Fig. II.4. Mkn 370 ( $D=11.2 \mathrm{Mpc})$. For explanations of symbols and labels, see Fig. II.1. a) $J$ band image and isophotes. b),c) Surface brightness and color profiles.

high continuum level, with absorption features that witness the substantial underlying population of older stars.

The finding that knot $b$ shows no appreciable old stellar background in NIR images, but is dominated by nebular emission, does not suggest that this source is the remnant of a former galaxy which has merged with Mkn 370 in the past. The latter idea is neither supported by the moderate velocity difference between $a$ and $b\left(40 \mathrm{~km} \mathrm{~s}^{-1}\right.$, Nordgren et al. 1995), which is of the order of typical velocity dispersions found the in H I components of BCDs (van Zee et al. 2001). Instead, the double-nucleus morphology of Mkn 370 is apparently attributable to extranuclear SF activity in knot b.

The LSB population, traceable on our images out to $R^{*} \approx 50^{\prime \prime}$, provides about $80 \%$ of the $J$ light. Its NIR colors (Table II.3), as well as the integrated $B-J$ color of 2.3 mag, computed using data from Cairós et al. (2002), are both consistent with an old stellar population. The LSB component shows in NIR wavelengths a roughly exponential slope, with a possible, but not significant slight flattening for $R^{*} \lesssim 30^{\prime \prime}$. The $J$ band scale length, $\alpha \lesssim 0.64 \mathrm{kpc}$, is significantly smaller than the $\alpha \sim 1 \mathrm{kpc}$ inferred from $B$ band data (Cairós et al. 2002). This difference is most likely due to the multiple extranuclear $\mathrm{H} \alpha$-emitting sources, distributed roughly along the optical major axis of Mkn 370 (Nordgren et al. 1995, Cairós et al. 2002). Similar to what is discussed for Mkn 600 (Sect. II.4.7), these sources may contaminate the LSB emission at large radii especially in optical wavelengths, artificially increasing the scale length of the old population. 

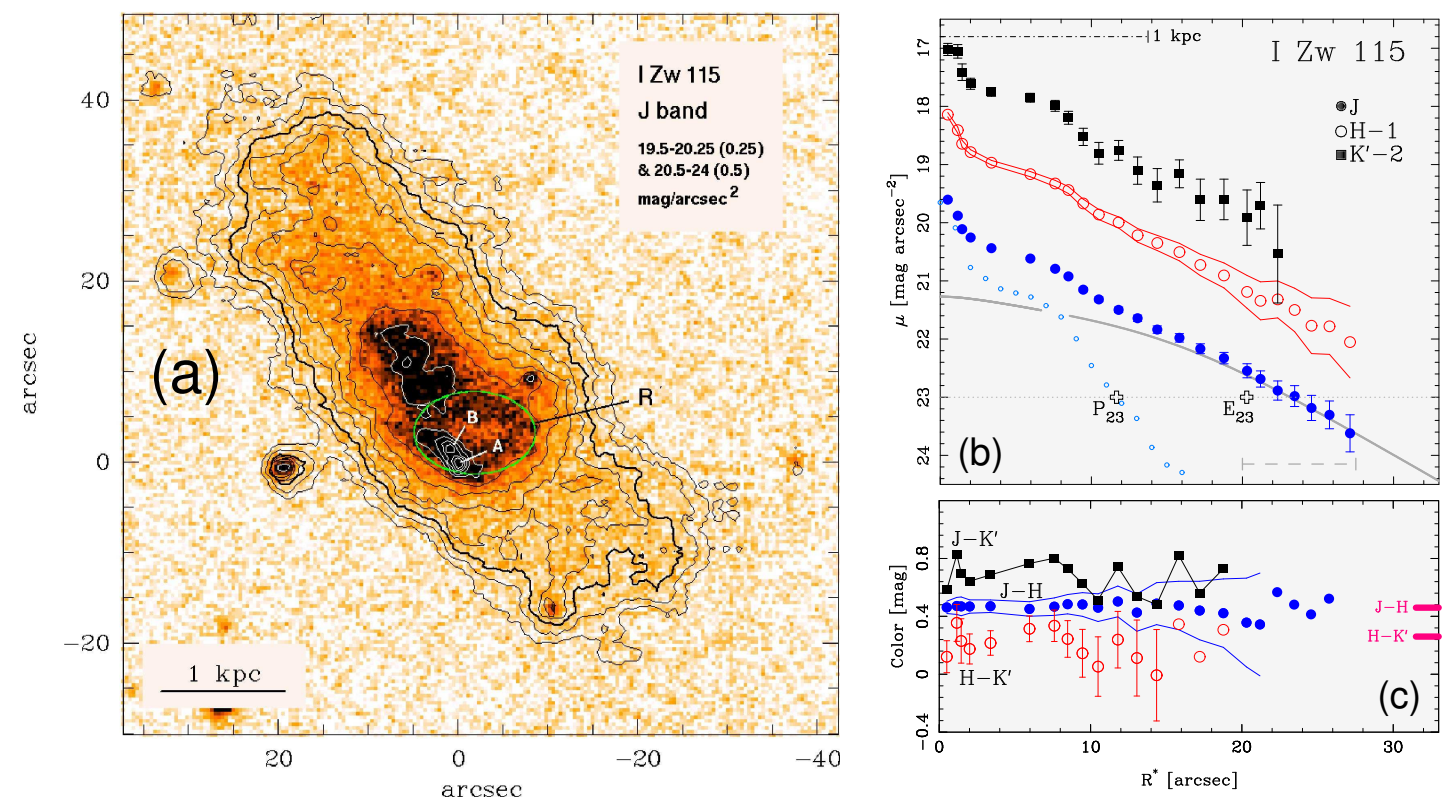

Fig. II.5. I Zw 115 ( $D=15$ Mpc). For explanations of symbols and labels, see Fig. II.1. (a): $J$ band image and isophotes. (b),(c): Surface brightness and color profiles. The thick grey line shows a fit to the host galaxy using a modified exponential distribution Eq. (II.2) with $b, q=2.4,0.80$.

\section{II.4.5 I Zw 115 (UGC 09893)}

This iI - classified (LT86) BCD ( $\left.\mathrm{M}_{B}=-16.4\right)$ was described in the Atlas of interacting galaxies by Vorontsov-Vel'Yaminov (1977) as "a pair of coalescents". Optical surface photometry for this system was first presented in P96a, while further $B, R$ and $\mathrm{H} \alpha$ images have recently been published in the catalogue of Blue Compact Dwarf Galaxies by GMP03.

I Zw 115 presents a peculiar morphology, displaying several intensity maxima within an LSB component which, other than in the majority of BCDs, shows boxy outer isophotes. P96a conjectured that this could be a signature of a dynamically unrelaxed underlying stellar component. The derived $B-J$ color the LSB host galaxy, $\sim 1.8 \pm 0.3 \mathrm{mag}$, is consistent with an age of a few Gyr. The brightest region, which spatially coincides in the optical and NIR broad-band frames, is located towards the south-west of the LSB component and is resolved into two smaller regions in the NIR images, labeled $A$ and $B$ in Fig. II.5. The latter regions seem to delineate, together with several other condensations, a ring-like structure ("R" in Fig. II.5a) with a projected diameter of $\sim 500 \mathrm{pc}$ in southeast-northwest direction. Northeast of this ring-like structure, several maxima are detected, reminiscent of the tails of SF regions observed in "cometary" BCDs. However, $\mathrm{H} \alpha$ emission in I Zw 115 is primarily confined to one single SF region, which is displaced $\sim 15^{\prime \prime}$ northeastwards with respect to $\mathrm{a}$ and $\mathrm{b}$ (see the maps by GMP03).

The SBPs show at large radii an exponential decay, with a $J$ band scale length of $\approx 530 \mathrm{pc}$, compatible to the one derived from optical data (P96a, transformed to the 
distance adopted in this paper). The type V LSB profile detected by the latter authors is confirmed by our analysis, which, however, implies a stronger central depression $q$ and larger cutoff radius $b \alpha$ than those inferred in the optical. Using the approach described in Sect. II.3, we can constrain the parameters of a med to $(b, q) \approx 2.4,0.80$.

\section{II.4.6 Mkn 5 (UGCA 130)}
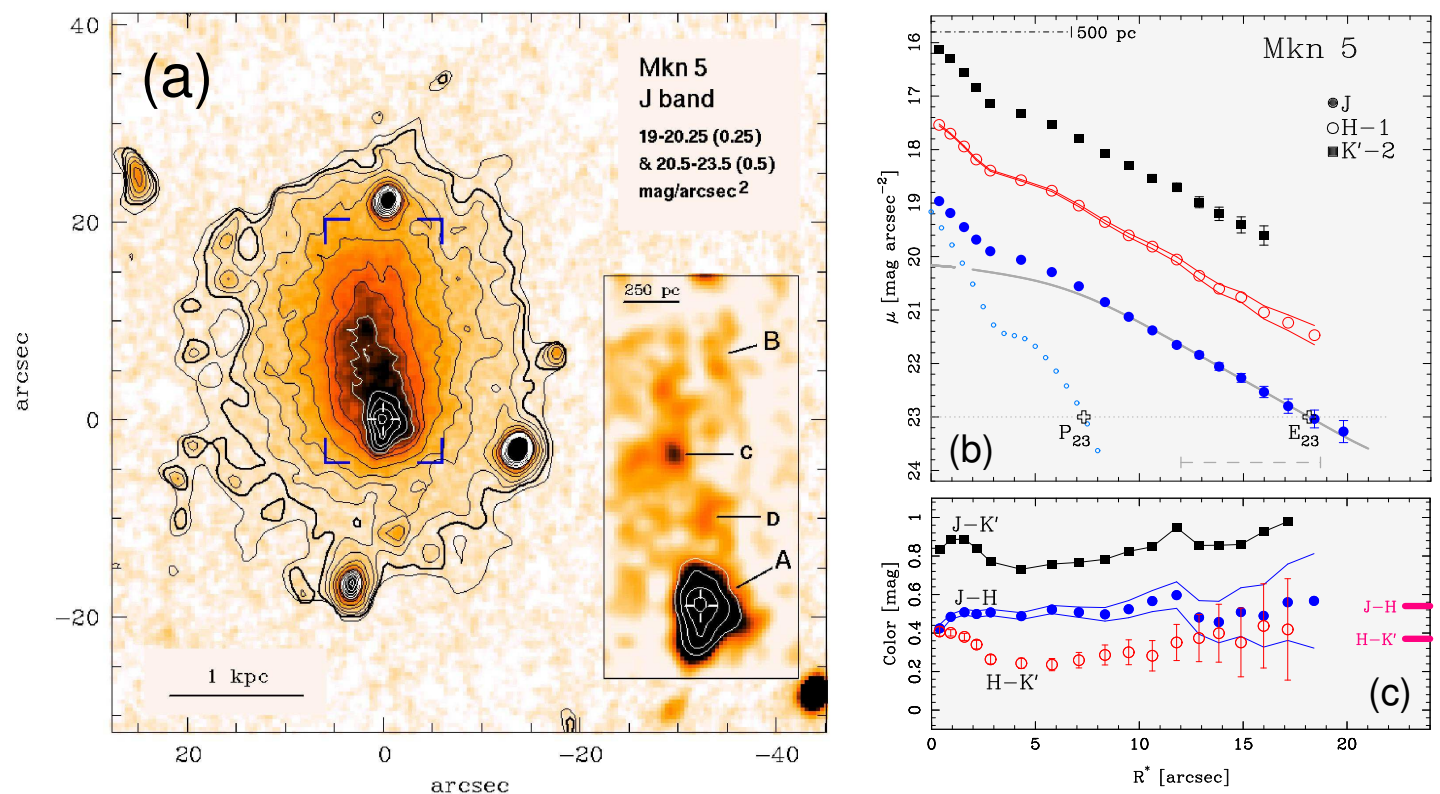

Fig. II.6. Mkn 5 ( $D=15.3 \mathrm{Mpc})$. For explanations of symbols and labels, refer to Fig. II.1. a) $J$ band image and isophotes. b),c) Surface brightness and color profiles. The thick grey line shows a fit to the LSB host galaxy using a modified exponential distribution Eq. (II.2) with $b, q=1.4,0.65$.

While Mkn 5 can be considered an average BCD with respect to its absolute magnitude $\left(M_{B}=-15.7\right)$, oxygen abundance $\left(\sim Z_{\odot} / 7\right.$; Izotov \& Thuan 1999$)$ and the regular, elliptical isophotes of its LSB host galaxy, it displays a peculiar, "cometary" morphology of its SF regions. This latter classification (LT86) denotes the presence of a dominant SF complex ("A" in Fig. II.6a) towards one end of an elongated stellar host galaxy, with fainter SF regions distributed along the optical major axis. Remarkably, the "iI,C" BCDs, selected by this morphology of the SF regions, typically display a more elongated, irregular host galaxy than that observed in Mkn 5 (see Noeske et al. 2000).

The dominant SF region A shows WR features (Conti 1991) and is the locus of intense and moderately extended nebular emission $(\mathrm{EW}(\mathrm{H} \alpha) \gtrsim 60 \AA$; Noeske 1999). The colors of this knot $(U-B=-0.78, B-V=0.49, V-R=0.18, V-I=0.19$ within a $4^{\prime \prime}$ aperture) are compatible with a burst age $\leq 4$ Myr (Cairós 2000). Note that $B-V$ appears relatively red due to the contribution of strong nebular line emission. 
Among the SF regions forming the "tail" northwards of A, the source $\mathrm{C}$ (Fig. II.6a) is the brightest in the NIR, in contrast to optical images, where this knot is only marginally detected. The second-brightest source in the optical, $B$, situated $\sim 20^{\prime \prime}$ north of $\mathrm{A}$, is barely seen even on contrast-enhanced $J$ images. The $\mathrm{H} \alpha$ equivalent width at this position is relatively low, $\sim 15 \AA$, suggesting that its blue colors are due to a young stellar population, rather than strong nebular emission.

The NIR surface brightness profiles reveal an extended plateau feature at high to intermediate intensity levels, similar to optical ones (C01a). The exponential LSB profile, observed in the outskirts of Mkn 5, flattens for small radii and can be well approximated by a med with $(b, q)=1.4,0.65$. The $J$ band exponential scale length, $\alpha \approx 5^{\prime \prime}$ $(0.37 \mathrm{kpc})$, is in good agreement with the results by Noeske et al. (2001b) and C01a.

\section{II.4.7 Mkn 600}
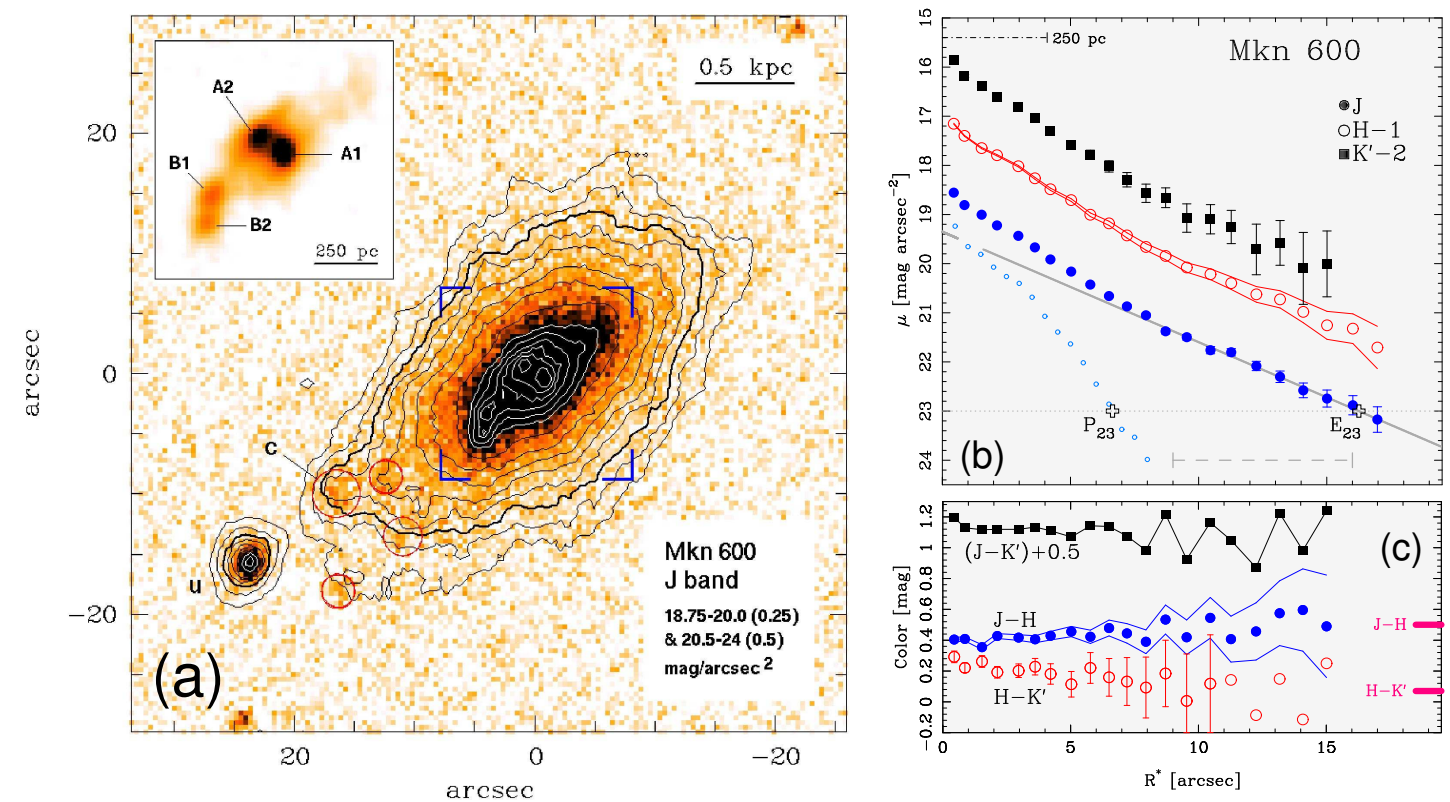

Fig. II.7. Mkn 600 ( $D=12.6$ Mpc). For explanations of symbols and labels, see Fig. II.1. a) $J$ band image and isophotes. The circles at the SE of the galaxy's main body mark extranuclear knots, one of which (C) was studied in e.g. C01b. The extended red object ' $u$ ', described by the same authors and probably not belonging to Mkn 600, is marked. b),c) Surface brightness and color profiles.

Star-forming activity in this $\mathrm{iE} \mathrm{BCD}\left(\mathrm{M}_{B}=-15.4, \mathrm{C} 01 \mathrm{~b} ; 12+\log \mathrm{O} / \mathrm{H}=7.83\right.$, Izotov\& Thuan 1999, Cairós 2000) occurs predominantly in its inner part $\left(R^{*} \leq 8^{\prime \prime}\right)$, where two distinct maxima, $A$ and $B$, are seen on optical images $(\mathrm{C} 01 \mathrm{~b})$. On the NIR images, each of the latter regions is resolved into two condensations, respectively denoted $A 1$, $A 2$ and B1, B2 in Fig. II.7a. The ongoing SF activity in both $A$ and $B$ is indicated by deep $\mathrm{H} \alpha$ images $(\mathrm{C} 01 \mathrm{~b})$ which show that either region coincides with local excesses in $\mathrm{H} \alpha$ line and $\mathrm{H} \alpha$ equivalent width maps. Both colors and $\mathrm{H} \alpha$ equivalent widths of $\mathrm{A}$ 
and B point to burst ages $<5 \mathrm{Myr}$ (Cairós 2000). Optical and NIR data reveal at fainter surface brightness levels a regular LSB host galaxy which extends out to $R^{*} \sim 30^{\prime \prime}$ and displays roughly elliptical isophotes. The colors of the latter component $(V-R \approx 0.3$, $V-I \approx 0.8, \mathrm{C} 01 \mathrm{a}$; for NIR colors, see Table II.3) are indicative of a several Gyr old stellar population.

A chain of fainter knots extends southeastwards from the center of Mkn 600, and connects with a moderately bright, $\mathrm{H} \alpha$-emitting source (region C in Fig. II.7). Source C, marginally detected on the NIR frames, emits strongly in $\mathrm{H} \alpha(E W(\mathrm{H} \alpha) \approx 590 \AA)$, and presents colors slightly bluer than the central knots $(U-B=-0.64,-0.72$ and -0.86 for knots $\mathrm{a}, \mathrm{b}$ and $\mathrm{c}$, respectively), suggesting a possible propagation of SF activity.

The hypothesis that the peculiar distribution of SF activity in Mkn 600 may be the result of an ongoing or recent interaction cannot be discarded, given the presence of a nearby H I companion (Taylor et al. 1993). Our NIR images reveal no stellar counterpart of this object down to a surface brightness limit of $\approx 23 \mathrm{Jmag} / \square^{\prime \prime}$.

As is evident from Fig. II.7a, the influence of the aforementioned extranuclear SF region $\mathrm{C}$ is relatively small in the NIR. This is also the case for other condensations in the vicinity of c (circles in Fig.II.7a). Surface brightness profiles, derived after subtraction of these sources, do therefore not notably differ from those derived from original images. For $R^{*}>9$ arcsec, the SBPs were approximated by an exponential distribution. The NIR scale lengths we derive $\left(\alpha \approx 4.8^{\prime \prime}\right)$ are slightly smaller than those derived by C01a from optical data ( $\left.\alpha \approx 5^{\prime \prime} .5\right)$. This difference is probably due to the stronger contribution of region $\mathrm{C}$ in optical bands (see also Sect. II.4.4).

The integral colors we infer for Mkn 600 can hardly be reconciled with those by Doublier et al. (2001). These authors report an integral $J-K$ color of $\sim 1$ mag, much redder than the $J-K$ of 0.65 we derive.

\section{II.4.8 NGC 6789 (UGC 11425)}

NGC 6789, an intrinsically faint $\left(\mathrm{M}_{B}=-14.3\right.$, Drozdovsky et al. 2001) galaxy with an $\mathrm{iE}$ morphology, is located at a distance of about 3.6 Mpc (Drozdovsky et al. 2001) and therefore belongs to the most nearby BCD candidates known to date, together with IC 10 (Richer et al. 2001) and IC 4662 (N03a). Karachentsev \& Makarov (1998) found NGC 6789 to be very isolated, situated towards the local void.

Deep optical imaging (Drozdovsky \& Tikhonov 2000) of this system revealed properties typical among BCDs, in particular an inner $\left(R^{*} \sim 150 \mathrm{pc}\right)$, high surface-brightness ionizing stellar population and a red $(V-I \approx 0.9 \mathrm{mag})$ underlying LSB host, extending out to at least $0.6 \mathrm{kpc}$.

Recent HST/WFPC2 observations (Drozdovsky et al. 2001) allowed to resolve this galaxy into more than 15000 stars, and to derive a minimum age of $1 \mathrm{Gyr}$ from colormagnitude diagrams, in agreement with the red $V-I$ LSB colors.

Inspection of the $J$ SBP slope in the radius range $25^{\prime \prime} \lesssim R^{*} \lesssim 38^{\prime \prime}$ shows that the LSB emission of NGC 6789 follows a type V profile, which was fitted by a med with a cutoff radius of $3.3 \alpha$ and central depression of $q \approx 0.7$. The scale length we derive in the outer regions, $\alpha \approx 200 \mathrm{pc}$ (Table II.2), differs from that inferred by Drozdovsky 

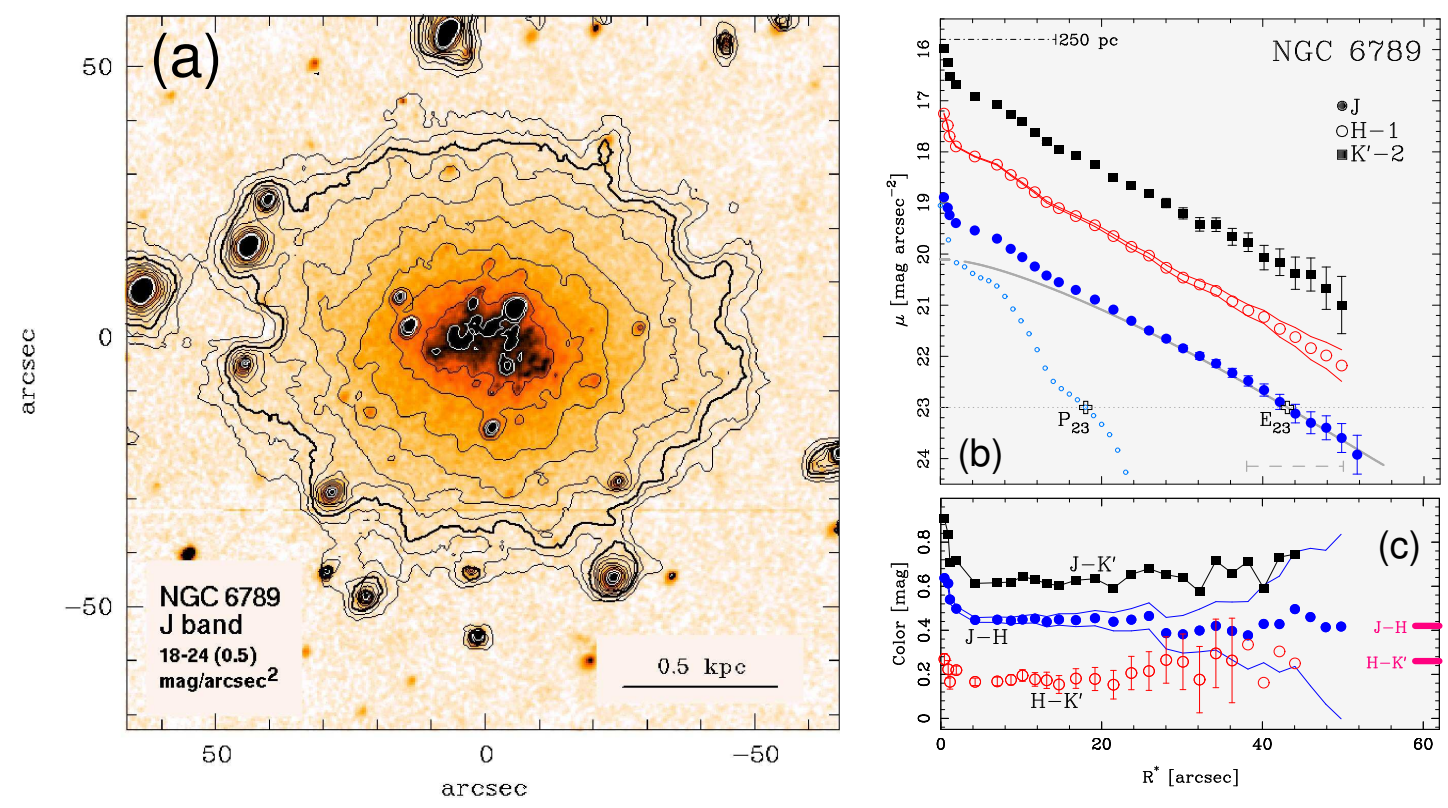

Fig. II.8. NGC 6789 ( $D=3.6 \mathrm{Mpc}$ ). For explanations of symbols and labels, see Fig. II.1. (a): $J$ band image and isophotes. (b),(c): Surface brightness and color profiles. The thick grey line shows a fit to the host galaxy using a modified exponential distribution Eq. (II.2) with $b, q=3.3,0.70$.

\& Tikhonov (2000) $(\alpha \approx 280 \mathrm{pc})$. Since the moderately flattening type V SBP of the LSB host was not revealed by optical data, the latter authors applied an exponential fit to the entire LSB emission (for $R^{*} \gtrsim 20^{\prime \prime}$ ), thereby including slightly flattened parts of the SBP inside the cutoff radius ( $\left.b \alpha \approx 38^{\prime \prime}\right)$. The exponential part of the med fitted to our $J$ band data has been adjusted to the steeper, outer exponential regime of the profile $\left(R^{*}>39^{\prime \prime}\right)$.

The exponential scale lengths and extrapolated central surface brightnesses inferred for NGC 6789, both by Drozdovsky \& Tikhonov (2000) and in this paper (see Table II.2), indicate a compact structure of the stellar host. This further supports the view that NGC 6789 displays properties typical of BCDs, rather than those of more diffuse dwarf irregulars.

\section{II.4.9 Mkn 324 (UGCA 439)}

Mkn $324\left(\mathrm{M}_{B}=-16.4, \mathrm{C} 01 \mathrm{~b}\right)$ is a compact iE BCD which displays two distinct SF regions close to its geometrical center, referred to by Mazzarella \& Boroson (1993) as $\mathrm{a}$ and $\mathrm{b}$ (Fig. II.9a) and studied by the same authors.

Surface photometry in the optical has been previously presented by Doublier et al. 1997,1999 and C01a. The latter authors also computed optical color maps, and disclosed on deep $\mathrm{H} \alpha$ images a complex morphology of the ionized gas, most notably a large supershell expanding to the northwest of the starburst region. The $\mathrm{H} \alpha$ intensity was found to peak in between the pair of bright star-forming regions $a$ and $b(\mathrm{C} 01 \mathrm{~b}$, 

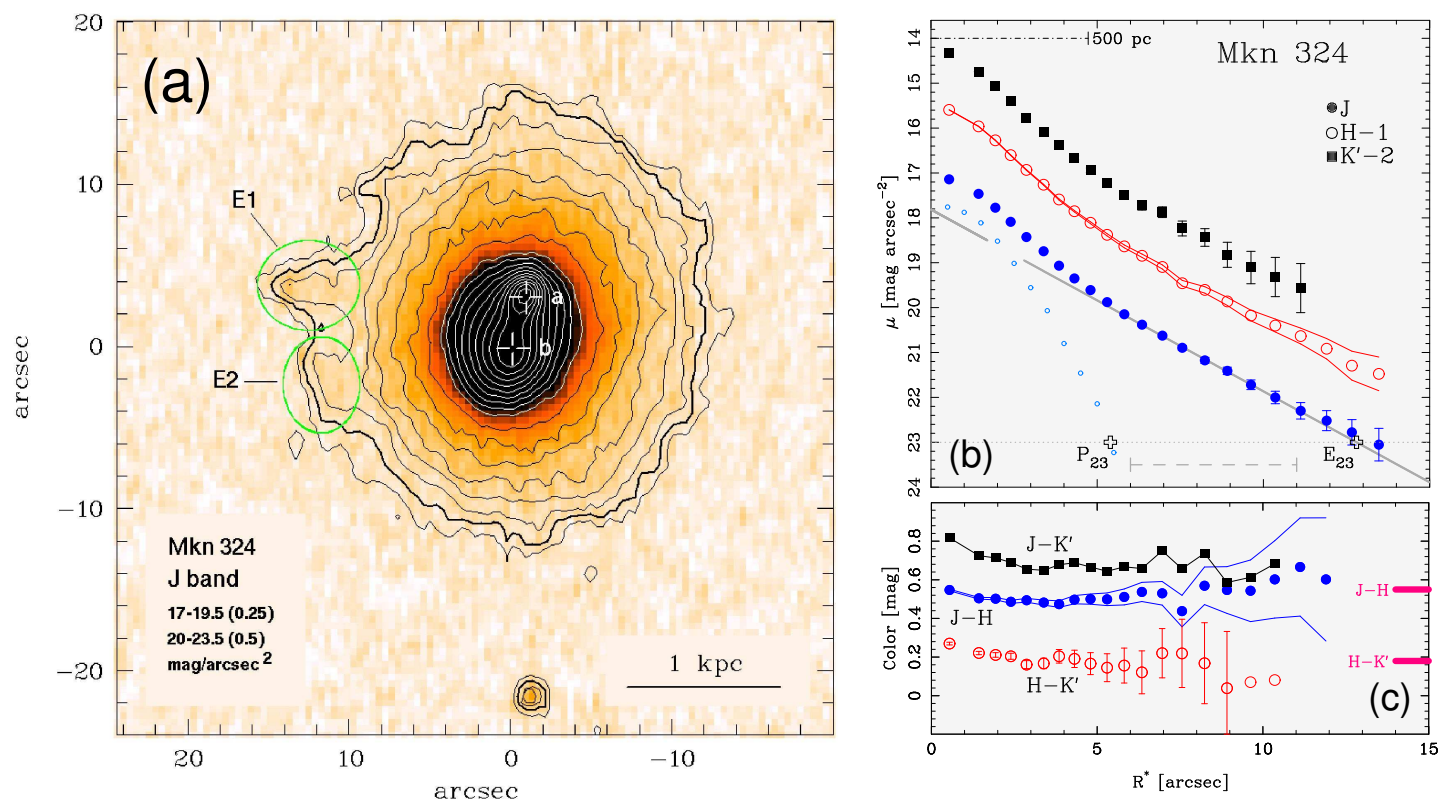

Fig. II.9. Mkn 324 ( $D=21.8 \mathrm{Mpc}$ ). For explanations of symbols and labels, refer to Fig. II.1. a) $J$ band image and isophotes. The star-forming knots a and b (Mazzarella \& Boroson 1993) are labeled. E1 and E2 mark the diffuse sources adjacent to the east of the LSB component (see text). b),c) Surface brightness and color profiles.

Petrosian et al. 2002). In its LSB periphery, Mkn 324 is delimited by regular outer isophotes, and shows no conspicuous signatures of a strong gravitative perturbation.

It appears nevertheless possible that the starburst activity of this system has been triggered by a distant low-mass companion. High-resolution H I VLA maps (van Zee et al. 2001) have recently unveiled a gas cloud with a mass of $\sim 2 \times 10^{8} M_{\odot}$, approximately $115 \mathrm{kpc} \mathrm{NW}$ of the BCD (values transformed to the distance adopted in this paper), with a projected velocity difference of $\sim 100 \mathrm{~km} \mathrm{~s}^{-1}$ to Mkn 324. A followup analysis of this system by Cairós et al. (2003b) has revealed an intrinsically faint, low-surface-brightness optical counterpart to this H I source. In this respect, it appears noteworthy that the H I component of Mkn 324 shows an overall solid-body rotation, though with conspicuous kinematical peculiarities, reminiscent of tidal tails (van Zee et al. 2001). 2D studies of the ionized gas component of the BCD (Petrosian et al. 2002) have shown a largely chaotic velocity field, with some underlying regular pattern.

Our surface photometry reveals in all NIR bands an exponential LSB distribution in the radius range $5^{\prime \prime} .9 \lesssim R^{*} \lesssim 11^{\prime \prime}$, with a moderately strong starburst emission. For this outer LSB component, we derive an exponential $J$ band scale length of $\approx 280 \mathrm{pc}$, in agreement with the $I$ band scale length derived by C01a. Some excess emission above the exponential SBPs for $R^{*}>11^{\prime \prime}$ is presumably due to an imperfect subtraction of diffuse sources adjacent to the eastern edge of the LSB component (sources E1 and E2 in Fig. II.9a).

The possibility that this excess above the exponential fit at large radii is a signature of a Sérsic law with an exponent $\eta \gtrsim 1$ could be dismissed. Sérsic fits over different radii in the interval $5^{\prime \prime} .9 \leq R^{*} \leq 15^{\prime \prime}$, yielding exponents $\eta$ between 1.4 and 2 , could not 
be extrapolated inwards without exceeding the observed SBPs at small radii, i.e., they fall short of describing the SBP of the LSB component.

\section{II.4.10 Mkn 450 (UGC 08323)}
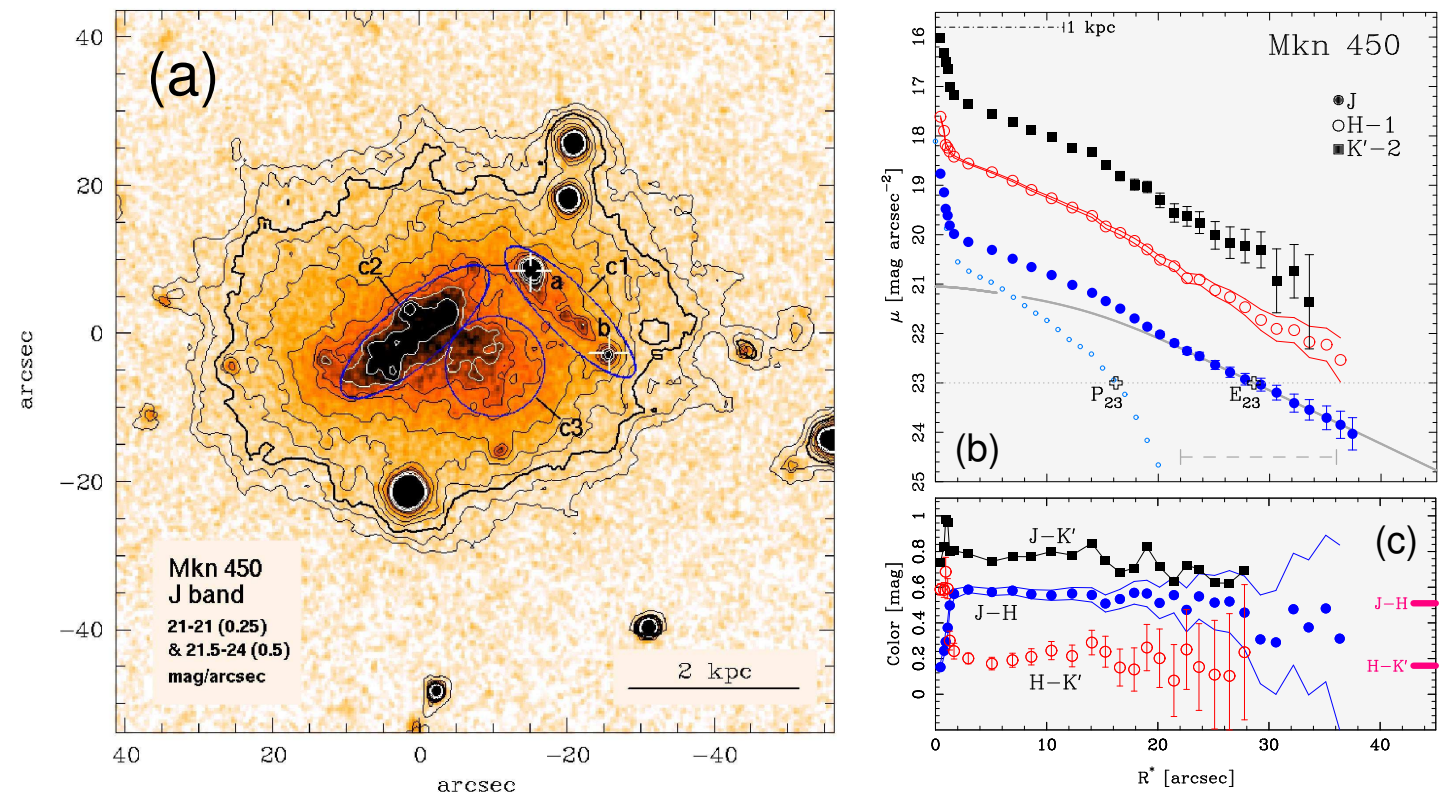

Fig. II.10. Mkn 450 ( $D=17.9$ Mpc). For explanations of symbols and labels, refer to Fig. II.1. a) $J$ band image and isophotes. The bright star-forming knots $\mathrm{a}$ and $\mathrm{b}$ are labeled. $\mathrm{c} 1 \ldots$ c3 mark distinct star-forming complexes described in the text. b),c) Surface brightness and color profiles. The thick grey line shows a fit to the host galaxy using a modified exponential distribution Eq. (II.2) with $b, q=1.5,0.65$.

Star-forming regions within this $\mathrm{iE} \mathrm{BCD}$ are distributed over a large surface fraction of its elliptical stellar LSB host. Individual condensations appear to be arranged within 3 distinct associations. Close to the geometrical center of the LSB host, an elongated structure (c2 in Fig. II.10) with an extent of roughly $2 \mathrm{kpc}$ is seen along the southeastnorthwest direction. It is displaced relative to the optical major axis of the galaxy, which roughly runs in east-west direction. Adjacent to the southwestern edge of $\mathrm{c} 2$, a fainter complex of knots, c3, can be discerned. A third strip of star-forming knots, c1, is seen NW of regions $\mathrm{C} 2$ and $\mathrm{c3}$, oriented roughly perpendicular to the former. The latter feature hosts the brightest individual condensations, $a$ and $b$ in Fig. II.10a. The photometric properties of either source (a: $m_{J} \sim 17 \mathrm{mag}, J-H \sim 0.3 \mathrm{mag}, H-K^{\prime} \sim$ $0.6 \mathrm{mag}$; b: $\left.m_{J} \sim 18 \mathrm{mag}, J-H \sim 0.2 \mathrm{mag}, H-K^{\prime} \sim 0.6 \mathrm{mag}\right)$, derived after correction for the surrounding continuum, point to young stellar populations and strong contributions of ionized gas. Recent $\mathrm{H} \alpha$ maps by GMP03 confirm that SF activity in Mkn 450 is largely confined to regions $\mathrm{a}$ and $\mathrm{b}$, while only faint $\mathrm{H} \alpha$ emission is present at the location of the features $\mathrm{c} 2$ and $\mathrm{c} 3$. 
The alignment of the morphological complex $\mathrm{C} 1$ with respect to the LSB host is untypical among $\mathrm{iE} \mathrm{BCDs;} \mathrm{usually,} \mathrm{such} \mathrm{elongated} \mathrm{sequences} \mathrm{of} \mathrm{SF} \mathrm{regions} \mathrm{are} \mathrm{found}$ closer to the center of such objects, at position angles rather parallel than perpendicular to the major axis of the LSB host (see e.g. the morphological catalogs by Telles et al. 1997, C01a, GMP03). Mkn 450 is a field BCD (Popescu et al. 1999) which shows no peculiarities among its other properties, such as its $B$ magnitude of $\mathrm{M}_{B}=-16.70$ (Vennik et al. 2000), and both a moderate metal-deficiency (1/5 $Z_{\odot}$, Garnett 1990) and star formation rate $\left(\sim 0.1 M_{\odot} \mathrm{yr}^{-1}\right.$; Popescu et al. 1999, value transformed to the distance adopted in this paper).

Subtraction of only the brightest irregular SF regions reveals a modest central flattening of the stellar LSB host galaxy, well described by a med with $(b, q)=(1.5,0.65)$.

\section{II.4.11 NGC 5058 (UGC 08345, MKN 786)}
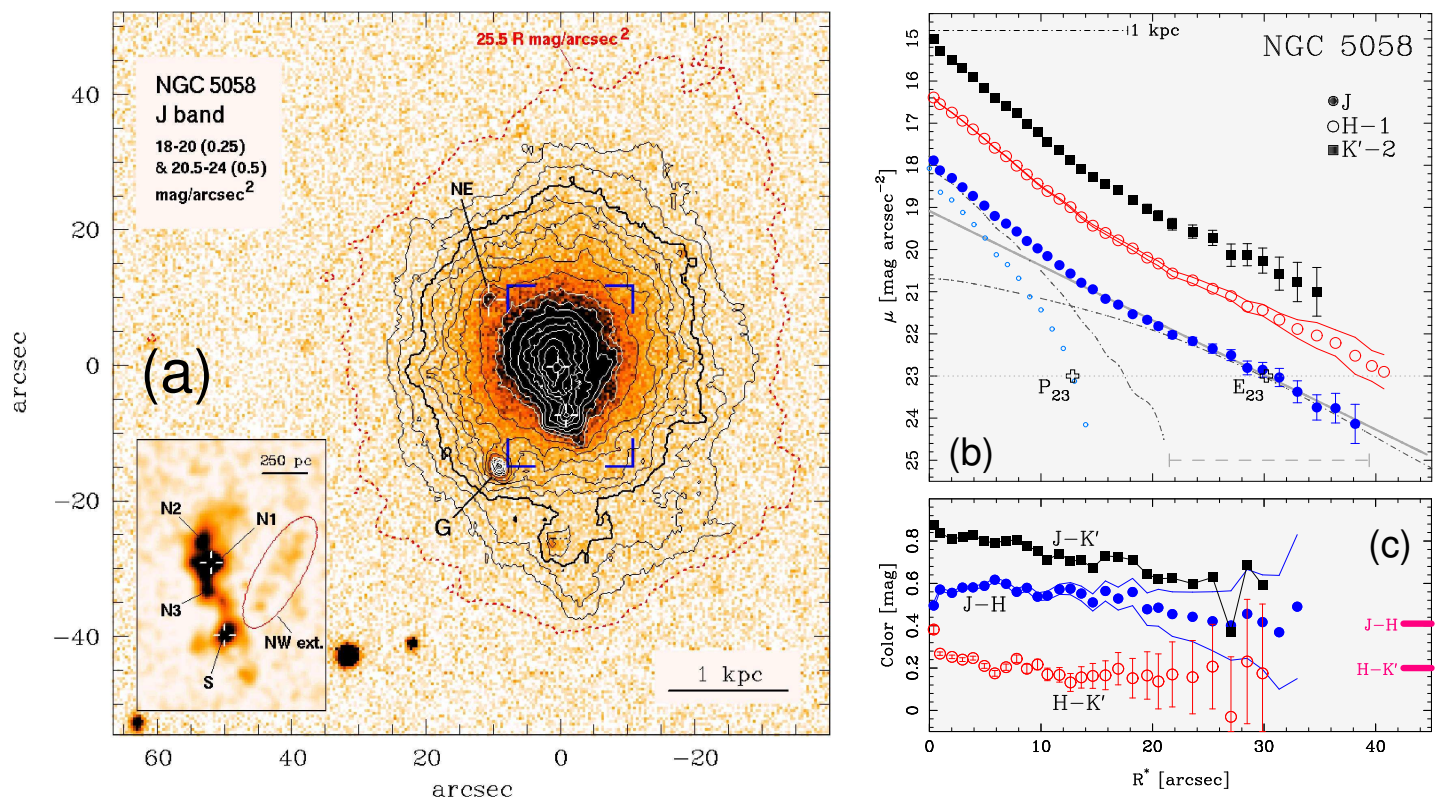

Fig. II.11. NGC $5058(D=11.6 \mathrm{Mpc})$. For explanations of symbols and labels, refer to Fig. II.1. a) $J$ band image and isophotes. The blue north-eastern knot (NE) and the red source to the southeast (G) are marked. Small white crosses mark the northern (N1) and southern (S) maxima. The inset shows a magnified, unsharp-masked image of the region bracketed in the main image. See the text. b),c): Surface brightness and color profiles. The thick solid line illustrates an exponential fit to the stellar LSB component in $J$ (cf. Sect. II.3), computed in the radius range indicated by the light gray, long-dashed bar at the bottom of the figure. The emission in excess to this fit is shown by the small open circles. The thin dash-dotted curve gives an example of alternative decomposition solutions, using a type $\mathrm{V}$ model for the LSB component (Eq. II.2 with $b, q=3.8,0.9$ ) . The residuals to this fit are also plotted as a dashdotted line. 
Deep images of this galaxy show two maxima (Fig. II.11a) within a common, extended LSB component, which at low surface brightness levels displays an asymmetric, curved morphology (see the $25.5 R \mathrm{mag} / \square^{\prime \prime}$ isophote in Fig. II.11a).

NGC 5058 was previously classified as a galaxy pair (KPG 370; Karachentsev 1972). The components of this pair (N \& S, Tifft 1982) are most likely identical to the maxima visible in Fig. II.11a, as can be inferred from their relative positions (projected separation 8.7 at a position angle $12^{\circ}$; Tifft 1982 for components $\mathrm{N} \& \mathrm{~S}$ ). We therefore adopted the latter nomenclature (cf. the inset in Fig. II.11a).

On contrast-enhanced NIR images, source $\mathrm{N}$ resolves into 3 distinct regions (N1, N2, N3 in Fig. II.11a). Fainter extensions emanate from this central source to the north, and southwards, connecting with source S. A third extension ("NW" in Fig. II.11a) extends northwestwards from the southern source $\mathrm{S}$. New $\mathrm{H} \alpha$ images by GMP03 show the SF activity to peak close to source S, and around a northeastern component, detached from the central regions ("NE" in Fig. II.11). The same data reveal only weak $\mathrm{H} \alpha$ emission along the NW extension, suggesting that this feature is not mainly due to nebular emission.

Since NGC 5058 is located at a small angular separation $\left(11^{\circ} .6\right)$ from the center of the Virgo Cluster, redshift-based distance determinations using Virgocentric infall models suffer from the Triple Value Problem (Teerikorpi et al. 1992). An alternative distance determination by the same authors, based on the Tully-Fisher relation, yields however a large distance of $37.7 \mathrm{Mpc}$, even outside the range of values allowed within the uncertainties of the Triple Value Problem (See Fig. 1 in Teerikorpi et al. 1992). We suggest that the H I velocity width that these authors use, $118 \mathrm{~km} \mathrm{~s}^{-1}$, may be partly enlarged by the southern component $\mathrm{S}$, which, by its velocity difference to component $\mathrm{N}\left(58 \mathrm{~km} \mathrm{~s}^{-1}\right.$, Tifft 1982), might be a kinematically distinct subunit of NGC 5058.

Dynamical distortions or even a past merger event in this galaxy appear also possible in view of the peculiar shape of the isophotes in the LSB regime, as mentioned above. Irregularities south of the center of the galaxy are traceable down to the limits of the $J$ band data $\left(\sim 24 \mathrm{Jmag} / \square^{\prime \prime}\right)$. These condensations in the southernmost outskirts of the galaxy show no obvious $\mathrm{H} \alpha$ emission (GMP03).

We adopt here a distance $D=11.6 \mathrm{Mpc}$, using the velocity of NGC 5058 with respect to the center of the Virgo Cluster (see N03a) and $H_{0}=75 \mathrm{~km} \mathrm{~s}^{-1} \mathrm{Mpc}^{-1}$; note that also for much larger distances, up to $D \lesssim 31.1 \mathrm{Mpc}$, the resulting absolute magnitude would be $>-18 B$ mag, still qualifying NGC 5058 as a BCD.

In view of the complex morphology at all surface brightness levels, and of widespread SF activity and further condensations, a decomposition of the surface brightness profiles is rendered problematic. For $R^{*}>21.6^{\prime \prime}$ i.e. outside appreciable irregular emission in the $J$ images, the SBPs can be approximated by an exponential, albeit some systematic differences (Fig. II.4.11b). The SBPs suggest however the presence of a type V SBP at large radii. An example decomposition by means of a med is shown in Fig. II.4.11b. The parameters of this function cannot be reliably constrained, given the extended influence of irregular and starburst emission, and the low $S / N$ levels $\left(\mu_{J} \sim 22 \ldots 23 \mathrm{mag} / \square^{\prime \prime}\right)$ at which we suspect the flattening to occur. The results of the 
profile decomposition given in Table II.2 therefore refer to the exponential fit to the LSB host.

Acknowledgement. Research by K.G.N. has been supported by the Deutsche Forschungsgemeinschaft (DFG) grants FR325/50-1 and FR325/50-2. P.P. and K.J.F. received support from the Deutsches Zentrum für Luft- und Raumfahrt e.V. (DLR) under grant 50 OR 9907 7. L.M.C. acknowledges support from the European Community Marie Curie Grant HPMF-CT-200000774. We are indebted to Dr. Tom Jarrett for kindly providing photometric data from the 2MASS survey prior to publication, which allowed to calibrate a part of the present sample. We thank Dr. Uta Fritze - v. Alvensleben, P. Anders, J. Bicker and J. Schulz for kindly providing the GALEV models. This research has made use of the NASA/IPAC Extragalactic Database (NED) which is operated by the Jet Propulsion Laboratory, CALTECH, under contract with the National Aeronautic and Space Administration. This publication makes use of data products from the Two Micron All Sky Survey, which is a joint project of the University of Massachusetts and the Infrared Processing and Analysis Center/California Institute of Technology, funded by the National Aeronautics and Space Administration and the National Science Foundation.

\section{References}

Binggeli, B., Cameron, L. M. 1991, A\&A, 252, 27

Cairós, L. M 2000, PhD Thesis, Universidad de La Laguna

Cairós, L. M., Caon, N., García-Lorenzo, B., Vílchez, J. M., \& Muñoz-Tuñón, C. 2002, ApJ, 577, 164

Cairós, L. M., Caon, N., Papaderos, P., Noeske, K. G., Vílchez, J. M., García Lorenzo, B., Muñoz-Tuñon, C. 2002a, submitted to the ApJ

Cairós, L. M., Caon, N., Vílchez, J. M., González-Pérez, J. N., \& Muñoz-Tuñón, C. 2001, ApJS, 136, 393 (C01b)

Cairós, L. M., Vílchez, J. M., González Pérez, J., Iglesias-Páramo, J. \& Caon, N. 2001a, ApJS, 133, 321 (C01a)

Cairós, L. M., Papaderos, P., Noeske, K. G., Caon, N. in preparation

Conti, P. S. 1991, ApJ, 377, 115

Cutri, R. M., et al. 2000, The 2MASS Explanatory Supplement

Deeg, H.-J., Duric, N., \& Brinks, E. 1997, A\&A, 323, 323

Deharveng, J.-M. et al. 1994, A\&A, 288, 413

de Vaucouleurs, G., de Vaucouleurs, A., Corwin, H. G., Buta, R. J., Paturel, G. \& Fouque, P. 1991, Third Reference Catalogue of bright Galaxies (Springer Verlag)

De Young, D. S. \& Heckman, T. M. 1994, ApJ, 431, 598

Doublier, V., Comte, G., Petrosian, A., Surace, C., \& Turatto, M. 1997, A\&AS, 124, 405

Doublier, V., Caulet, A., Comte, G. 1999, A\&AS, 138, 213

Doublier, V., Caulet, A., Comte, G. 2001, A\&A, 367, 33

Drozdovsky, I. O., Schulte-Ladbeck, R. E., Hopp, U., Crone, M. M. \& Greggio, L. 2001, ApJ, 551, L135

Drozdovsky, I. \& Tikhonov, N. 2000, A\&AS, 142, 347

Fanelli, M. N., O'Connell, R. W., \& Thuan, T. X. 1988, ApJ, 334, 665

Garnett 1990

Gerola, H., Seiden, P. \& Schulmann, L. 1980, ApJ, 242, 517

Gil de Paz, A., Zamorano, J.; Gallego, J., Domínguez, F. de B. 2000a, A\&AS, 145, 377 
Gil de Paz, A., Zamorano, J., Gallego, J. 2000b, A\&A, 361, 465

Gil de Paz, A., Madore, B.F., \& Pevonova, O. 2003, ApJS, in press (GMP03)

Guseva, N. G., Izotov, Y. I., \& Thuan, T. X. 2000, ApJ, 531, 776

Izotov, Y. I. \& Thuan, T. X. 1999, ApJ, 511, 639

Jarrett, T. H., Chester, T., Cutri, R., Schneider, S. \& Huchra, J. 2000, AJ, 119, 2498

Karachentsev, I. D. \& Makarov, D. I. 1998, A\&A, 331, 891

Karachentsev, I. D. 1972, Isolated Pairs of Galaxies Catalogue, 7, 3

Krüger, H., Fritze-v. Alvensleben, U., Loose, H.-H. 1995, A\&A, 303, 41

Loose, H. H. \& Thuan, T. X. 1986, Star Forming Dwarf Galaxies and Related Objects, 73 (LT86)

Mac Low, M. \& Ferrara, A. 1999, ApJ, 513, 142

Makarova, L. N., Karachentsev, I. D., \& Georgiev, T. B. 1997, Astronomy Letters, 23, 378

Mas-Hesse, J. M. \& Kunth, D. 1999, A\&A, 349, 765

Mazzarella, J. M. \& Boroson, T. A. 1993, ApJS, 85, 27

Noeske, K. G. 1999, Diploma Thesis, Universität Göttingen

Noeske, K. G., Guseva, N. G., Fricke, K. J., Izotov, Y. I., Papaderos, P., Thuan, T. X. 2000, A\&A, 361, 31 (Paper IV)

Noeske, K. G., Iglesias-Páramo, J., Vílchez, J. M., Papaderos, P., \& Fricke, K. J. 2001a, A\&A, 371, 806 (Paper VI)

Noeske, K. G., Cairós, L. M. Papaderos, P., Vílchez, J. M. \& Fricke, K. J. 2001b, Ap\&SS, 276, 901

Noeske, K. G., Papaderos, P., Cairós, L. M. \& Fricke, K. J. 2003a, A\&A, submitted (Paper I)

Nordgren, T. E., Helou, G., Chengalur, J. N., Terzian, Y., \& Khachikian, E. 1995, ApJS, 99, 461

Papaderos, P., et al. 2003, in prep.

Papaderos, P. 1998, PhD Thesis, University of Göttingen

Papaderos, P., Izotov, Yu.I., Noeske, K.G., Thuan, T.X., Fricke, K.J., 2001, in "Dwarf Galaxies and their Environment", eds K.S. de Boer, R.J. Dettmar, U. Klein, Shaker Verlag, p. 111

Papaderos, P., Izotov, Yu.I., Thuan, T.X., Noeske, K.G., Guseva, N.G., Fricke, K.J. 2002, $A \& A$, in press

Papaderos, P., Loose, H.-H., Fricke, K. J., Thuan, T. X. 1996b, A\&A,314, 59 (P96b)

Papaderos, P., Loose, H.-H., Thuan, T.X., Fricke, K.J., 1996a, A\&AS 120, 207 (P96a)

Petrosian, A. R., Movsessian, T., Comte, G., Kunth, D., \& Dodonov, S. 2002, A\&A, 391, 487

Popescu, C. C., Hopp, U. \& Rosa, M. R. 1999, A\&A, 350, 414

Richer, M. G., Bullejos, A., Borissova, J., et al. 2001, A\&A, 370, 34

Salzer, J.J., McAlpine, G.M., Boroson, T.A. 1989b, ApJS, 70, 479

Schaerer, D., Contini, T., \& Pindao, M. 1999, A\&AS, 136, 35

Schulte-Ladbeck, R. E., Hopp, U., Greggio, L., Crone, M. M., \& Drozdovsky, I. O. 2001, AJ, 121, 3007

Silich, S. \& Tenorio-Tagle, G. 2001, ApJ, 552, 91

Taylor, C. L., Brinks, E., Pogge, R. W. \& Skillman, E. D. 1994, AJ, 107, 971

Taylor, C., Brinks, E. \& Skillman, E. D. 1993, AJ, 105, 128

Telles, E., Melnick, J. \& Terlevich, R. 1997b, MNRAS, 288, 78

Teerikorpi, P., Bottinelli, L., Gouguenheim, L. \& Paturel, G. 1992, A\&A, 260, 17

Thuan, T.X. \& Martin, G. 1981, ApJ, 247, 823

Thuan, T. X., Izotov, Y. I., Lipovetsky, V. A. 1996, ApJ, 463, 120 
Tifft, W. G. 1982, ApJS, 50, 319

Tully, R. B. 1988, Nearby Galaxies Catalog (Cambridge University Press)

van Driel, W., Arnaboldi, M., Combes, F., \& Sparke, L. S. 2000, A\&AS, 141, 385

van Zee, L., Salzer, J. J., \& Skillman, E. D. 2001, AJ, 122, 121

van Zee, L., Skillman, E.D. \& Salzer, J.J. 1998, AJ, 116, 1186

Vennik et al. 2000

Viallefond, F. \& Thuan, T. X. 1983, ApJ, 269, 444

Vorontsov-Vel'Yaminov, B. A. 1977, A\&AS, 28, 1

Whitmore, B. C., Lucas, R. A., McElroy, D. B., Steiman-Cameron, T. Y., Sackett, P. D., \& Olling, R. P. 1990, AJ, 100, 1489 


\title{
New insights to the photometric structure of Blue Compact Dwarf Galaxies from deep Near-Infrared studies $^{0}$
}

\author{
III. The photometric structure of the stellar host galaxy and its \\ relations to star-forming activity
}

\author{
K.G. Noeske, P. Papaderos, L.M. Cairós, K.J. Fricke \\ Universitäts-Sternwarte, Geismarlandstraße 11, D-37083 Göttingen, Germany
}

Summary. We analyze the results of Near Infrared (NIR) surface photometry of a sample of 31 Blue Compact Dwarf Galaxies (BCDs) and 2 luminous Blue Compact Galaxies, published in previous papers of this series. Compared to the visible light, NIR data allow for a better separation of the starburst emission from the light distribution of the old underlying stellar low-surface brightness (LSB) host galaxy. We study the photometric structure of the latter stellar component, and explore its possible influence on the global star-forming process. The exponential scale lengths $\alpha_{E}$ and extrapolated central surface brightnesses $\mu_{E, 0}$ of the stellar host galaxies, derived from exponential fits to their outer regions, are generally in agreement in optical and NIR wavelengths, confirming the previous conclusion that stellar hosts of BCDs possess a systematically more compact stellar distribution than dwarf irregulars. For more than $50 \%$ of the BCDs, we detect "type V" surface brightness profiles (SBPs) of the stellar host galaxy, i.e. exponential profiles flattening within a galactocentric radius of 1 to 3 scale lengths. In view of strong observational biases against the detection of such profiles in BCDs, this fraction is most likely significantly underestimated. The physical origin of such profiles in spheroidal dwarf galaxies has not been investigated thus far. We modelled type $\mathrm{V}$ profiles using a modified exponential distribution (Papaderos et al. 1996a) which, in addition to the extrapolated central surface brightness $\mu_{E, 0}$ and scale length $\alpha_{E}$, depends on a cutoff radius $b \alpha_{E}$ and a relative depression index $q$, relative to a standard exponential distribution. $b$ and $q$ show a tight non-linear relation among each other, yet no significant dependence on the absolute magnitude $M_{\mathrm{E}}$ and structural parameters $\mu_{E, 0}$ and $\alpha_{E}$ of the stellar host. We derive a mean starburst-to-LSB luminosity ratio of $\sim 0.6$ in $J$ and $\sim 1.8$ in $B$, and estimate the degree to which the latter ratio might be affected by the non-detection of type V LSB profiles in optical data. Combining empirical results on the radius and fractional areas of the starburst component in BCDs with previous estimates to the intrinsic luminosity density profile in their LSB host galaxies, we raise the hypothesis of a LSB host threshold density of the order of $\sim(0.4 \pm 0.2) M_{\odot} \mathrm{pc}^{-3}$, below which burst-like, collective star-forming activity is unlikely to occur. This simple hypothesis can reproduce remarkably well the observed systematic separation of BCDs from dwarf irregulars in the $\alpha_{E}-M_{\mathrm{E}}$ and $\mu_{E, 0}-M_{\mathrm{E}}$ parameter space, and can account in a qualitative manner for the relation between $M_{\mathrm{E}}$ and both the absolute and relative spatial extent of the starburst component within the LSB host galaxy. We suggest that the dependence of the starburst properties on the

\footnotetext{
0 To be submitted to Astronomy \& Astrophysics.
} 
stellar mass density of the LSB host galaxy might be explained by a modified Jeans-criterion that includes the influence of a compact stellar LSB background on the Jeans mass, similar to a model envisaged by Loose \& Fricke (1980).

\section{III.1 Introduction}

In this paper we discuss the photometric structure of Blue Compact Dwarf (BCD) galaxies, based on observational constraints from the study of a large sample of such systems in the Near Infrared (NIR; Noeske et al. 2003a, hereafter N03a, Cairós et al. 2003 (C03) and Noeske et al. 2003b (N03b)). Our goal is to systematically study the structural properties of the old stellar low-surface-brightness (LSB) host galaxy in BCDs, and to explore their possible influence on the starburst activity. For such an investigation one first needs to separate the light distributions of the young and old stellar populations of a BCD by decomposing surface brightness profiles (SBPs). Whereas such an analysis is rendered difficult by the extended starburst emission at optical wavelengths, it can be performed with a much better precision in the NIR (see N03a, C03).

The sample selection, observations and data reduction techniques have been described in N03a, C03 and N03b. In these studies we also give details about the derivation, analysis and decomposition of SBPs and color profiles, and study the size and total luminosity of the starburst and the LSB population of BCDs. In addition, we fitted different empirical functions to the LSB surface brightness profiles (hereafter $\mathrm{SBP}_{\mathrm{LSB}}$ ) of BCDs, such as the Sérsic law or a modified exponential distribution proposed in Papaderos et al. (1996a; P96a).

In the present paper, we compile and further analyze the results of the above listed surface photometry studies. Using the complete sample, complemented with $B$ band data from the literature, we compare the structural and photometric properties of the old and young stellar populations of BCDs in the visible and NIR range. Furthermore, we derive estimates to the stellar mass distribution of the old LSB host which contains practically the total stellar mass of a typical BCD and can therefore be important for understanding the overall dynamics of these systems. The relation between the stellar mass distribution and the occurrence, spatial extent and intensity of star-forming (SF) activity in BCDs are investigated in detail, and our results are discussed in view of a possible evolutionary link between dwarf irregulars (dIs) and BCDs. We also attempt a first systematic study of exponential $\mathrm{SBP}_{\mathrm{LSB}}$ which show a flattening at small galactocentric radii ("type V SBP $\mathrm{LSB}$ "). Our results (N03a,b) suggest that such profiles are relatively common among BCDs, but have been mostly missed in previous optical studies because of the dominant starburst emission. The exact form and physical origin of such profiles in the stellar LSB component of BCDs are both not yet investigated.

This paper is structured as follows: In Sect. III.2, we list the sample galaxies and the literature sources of the NIR and $B$ band photometry. In Sect. 3, we compare the structural properties of the $\mathrm{SBP}_{\mathrm{LSB}}$ in the $B$ band and the NIR, as derived from the commonly used exponential fits to the outer part of SBPs. A detailed study of inwardsflattening (type V) $\mathrm{SBP}_{\mathrm{LSB}}$ with respect to their frequency and the dependence of their 
characterizing parameters on other observables is presented in Sect. 4. In Sect. 5 we explore the relation between the starburst component and the structural properties of the underlying LSB host and, based on the present observational constraints, we revisit the hypothesis of an evolutionary link between BCDs and dIs. Our results are summarized in Sect. 6.

\section{III.2 The data set}

The NIR surface photometry data upon which the current analysis is based are compiled from N03a, C03 and N03b . Comparison data in the $B$ band were mostly taken from P96a and Cairós et al. 2001a.

The total sample of 33 objects is listed in Table III.1. Column 2 gives the morphological type of each $\mathrm{BCD}(\mathrm{iE} / \mathrm{nE} / \mathrm{iI} / \mathrm{iI}, \mathrm{C})$ according to classification scheme by Loose \& Thuan (LT86). "BCG" denotes Blue Compact Galaxies, i.e. objects significantly brighter than the commonly used absolute magnitude cutoff $(-18 \mathrm{~B}$ mag) for dwarf galaxies. In the third column, we give a qualitative description for the goodness of fits to the visible (outer) part of each $\mathrm{SBP}_{\mathrm{LSB}}$ in $\mathrm{N03a}$ and $\mathrm{N03b}$, and on the inferred shape of these $\mathrm{SBP}_{\mathrm{LSB}}: 1$ ) perfectly exponential $\mathrm{SBP}_{\mathrm{LSB}} 2$ ) approximated by an exponential, despite small systematic deviations; actual shape insecure 3) roughly exponential with

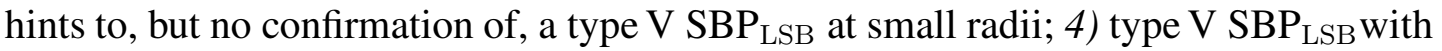
large uncertainties; 5) clear evidence for a type $\mathrm{V} \mathrm{SBP}_{\mathrm{LSB}}$. Both cases 4) and 5) were modelled by means of a modified exponential (Eq. III.18). Columns (4) and (5) list the literature sources of the $B$ band and NIR surface photometry.

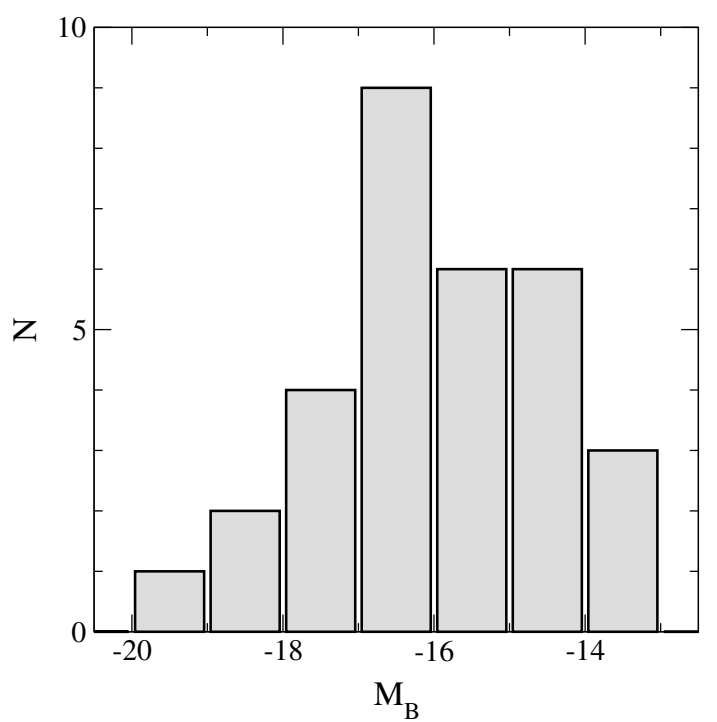

Fig. III.1. Histogram of the absolute $B$ band magnitudes of the sample galaxies

The sample covers all morphological types of BCDs except for the rare i0 BCD class. Nineteen galaxies belong to the most common $\mathrm{iE} / \mathrm{nE}$ class, with regular elliptical outer isophotes and 11 are iI objects, presenting irregular outer isophotes. Six 
Table III.1. Sample galaxies; see Sect. III.2

\begin{tabular}{|c|c|c|c|c|}
\hline $\begin{array}{l}\text { Object } \\
\text { (1) }\end{array}$ & $\begin{array}{l}\text { morph. } \\
\text { (2) }\end{array}$ & $\begin{array}{l}\text { SBP }_{\text {LSB }} \text { type } \\
\text { (3) }\end{array}$ & $\begin{array}{c}\operatorname{Ref}_{B} \\
(4)\end{array}$ & $\begin{array}{l}\operatorname{Ref}_{N I R} \\
\quad(5)\end{array}$ \\
\hline Mkn 297 & BCG & - & C01a & $\mathrm{C} 03$ \\
\hline I Zw 123 & $\mathrm{iE}$ & - & $\mathrm{C} 01 \mathrm{a}$ & $\mathrm{C} 03$ \\
\hline II Zw 71 & $\mathrm{iE}$ & - & $\mathrm{C} 01 \mathrm{a}$ & $\mathrm{C} 03$ \\
\hline II Zw 70 & iI & - & $\mathrm{C} 01 \mathrm{a}$ & $\mathrm{C} 03$ \\
\hline UM 462 & iI & - & C01a & $\mathrm{C} 03$ \\
\hline Mkn 36 & $\mathrm{iE}$ & - & $\mathrm{C} 01 \mathrm{a}$ & $\mathrm{C} 03$ \\
\hline Mkn 35 & $\mathrm{iE}$ & - & $\mathrm{C} 01 \mathrm{a}$ & $\mathrm{C} 03$ \\
\hline Mkn 33 & $\mathrm{iE}$ & - & $\mathrm{C} 01 \mathrm{a}$ & $\mathrm{C} 03$ \\
\hline Mkn 407 & $\mathrm{iE}$ & - & $\mathrm{C} 01 \mathrm{a}$ & $\mathrm{C} 03$ \\
\hline NGC 5058 & $\mathrm{iI} / \mathrm{M} ?$ & 3 & - & N03b \\
\hline Mkn 450 & $\mathrm{iE}$ & 5 & V00 & N03b \\
\hline NGC 6789 & $\mathrm{iE}$ & 4 & - & N03b \\
\hline Mkn 324 & $\mathrm{iE}$ & 1 & $\mathrm{C} 01$ & N03b \\
\hline Mkn 600 & $\mathrm{iE}$ & 3 & $\mathrm{C} 01 \mathrm{a}$ & N03b \\
\hline I Zw 115 & iI & 5 & P96a & N03b \\
\hline Mkn 370 & $\mathrm{iE}$ & 2 & $\mathrm{C} 01 \mathrm{a}$ & N03b \\
\hline Mkn 996 & $\mathrm{nE}$ & 1 & T96 & N03b \\
\hline Mkn 209 & $\mathrm{iE}$ & 3 & P96a & N03b \\
\hline Mkn 314 & $\mathrm{iE}$ & 3 & $\mathrm{C} 01 \mathrm{a}$ & N03b \\
\hline Mkn 5 & iI,C & 5 & $\mathrm{C} 01 \mathrm{a}$ & N03b \\
\hline IC 4662 & $\mathrm{iE}$ & 4 & - & N03a \\
\hline Mkn 1329 & iI,C & 5 & - & N03a \\
\hline Mkn 178 & $\mathrm{iE}$ & 2 & $\mathrm{P} 02 \mathrm{a}$ & N03a \\
\hline Tol 1214-277 & iI,C & 5 & F01 & N03a \\
\hline UM 448 & BCG & 3 & - & N03a \\
\hline Pox 4B & $\mathrm{iI} / \mathrm{dI}$ & 5 & - & N03a \\
\hline Pox 4 & iI & 4 & - & N03a \\
\hline Tol 1400-411 & iI,C & 5 & - & N03a \\
\hline He $2-10$ & $\mathrm{nE}$ & 1 & P98 & N03a \\
\hline UM 461 & iI & 5 & - & N03a \\
\hline Haro 14 & $\mathrm{iE}$ & 5 & M99 & N03a \\
\hline Tol 65 & iI,C & 5 & P99 & N03a \\
\hline Tol3 & $\mathrm{iE}$ & 1 & M99 & N03a \\
\hline
\end{tabular}

References: C01: Cairós et al. 2001a, C03: Cairós et al. 2003, F01: Fricke et al. 2001, M99: Marlowe et al. 1997, N03a: Noeske et al. 2003a, N03b: Noeske et al. 2003b, P96a: Papaderos et al. 1996a, P98: Papaderos 1998, P99: Papaderos et al. 1999, P02a: Papaderos et al. 2002, V00: Vennik et al. 2000 
objects from the latter subset show a cometary (iI,C) morphology, with their SF activity concentrated towards the one end of an elongated LSB host. Two sample galaxies (Mkn 297 and UM 448) fall into the BCG category, and Pox 4B, the faint companion of the iI BCD Pox 4, is a yet unclassified star-forming dwarf galaxy. Our sample shows a median absolute $B$ magnitude of -16.5 mag (Fig. III.1).

\section{III.3 The photometric structure of the LSB host of BCDs}

\section{III.3.1 Central surface brightness and exponential scale length}

An exponential model (Eqs. III.1\&III.2) is generally used to approximate the $\mathrm{SBP}_{\mathrm{LSB}}$ of BCDs (e.g. P96b, Marlowe et al. 1997, Telles et al. 1997, Salzer \& Norton 1999, Vennik et al. 2000, Cairós et al. 2001a), and the SBPs of most dwarf galaxies outside their central regions (Binggeli \& Cameron 1991, Patterson \& Thuan 1996, Parodi et al. 2002, Barazza et al. 2001, Makarova et al. 2002, van Zee 2000,2001). Given that optical surface photometry for dwarf galaxies is mostly available in the $B$ band, and in the NIR wavelength range $J$ data have typically the best quality, we use data in these two wavebands to compare the exponential fits to the $\mathrm{SBP}_{\mathrm{LSB}}$.

Throughout this paper, we will refer to the extrapolated central intensity and surface brightness of the exponential LSB model as $I_{0, \mathrm{E}}$ and $\mu_{E, 0}$, respectively, and to the exponential scale length as $\alpha_{E}$. The apparent and absolute magnitudes of the LSB component are denoted $m_{\mathrm{E}}$ and $M_{\mathrm{E}}$, respectively. The aforementioned quantities are related among each other as:

$$
\begin{array}{r}
I\left(R^{\star}\right)=I_{\mathrm{E}, 0} \exp \left(-\frac{R^{\star}}{\alpha_{\mathrm{E}}}\right) \\
\mu\left(R^{\star}\right)\left[\mathrm{mag}^{\star} \operatorname{arcsec}^{-2}\right]=\mu_{\mathrm{E}, 0}+1.086 \frac{R^{\star}}{\alpha_{\mathrm{E}}} \\
m_{\mathrm{E}}=\mu_{\mathrm{E}, 0}-5 \log \left(\alpha_{E}\left[^{\prime \prime}\right]\right)-2.00 \\
M_{\mathrm{E}}=\mu_{0, E}-5 \log \left(\alpha_{E}[\mathrm{pc}]\right)-23.57
\end{array}
$$

with $R^{\star}$ denoting the photometric radius.

Note that $M_{\mathrm{E}}$ has not been calculated throughout using Eq. (III.4); for BCDs showing a type $\mathrm{V}$ profile in their LSB component, we computed $M_{\mathrm{E}}$ by integrating the modified exponential (P96a; Eq. III.18, this paper) fit to the $\mathrm{SBP}_{\mathrm{LSB}}$. Two of the four free parameters of the latter model, $\alpha_{E}$ and $\mu_{E, 0}$, were obtained as usual, by fitting Eq. (III.4) to the outer exponential part of each $\mathrm{SBP}_{\mathrm{LSB}}$, outside the cutoff radius inside of which a type V SBP levels off.

Figure III.2 shows the distribution of $\mu_{E, 0}$ and $\alpha_{E}$ in the $B$ and $J$ bands, vs. the absolute $J$ magnitude $M_{\mathrm{E}, J}$ of the LSB component, for the objects from Table III.1. From the right panel of the figure it is evident that the $\alpha_{E}$ derived in the $J$ and in the $B$ band do, on average, not significantly differ. The scale lengths derived in $H$ and $K$ show a comparable agreement with those from the $B$ band, with mean ratios of 

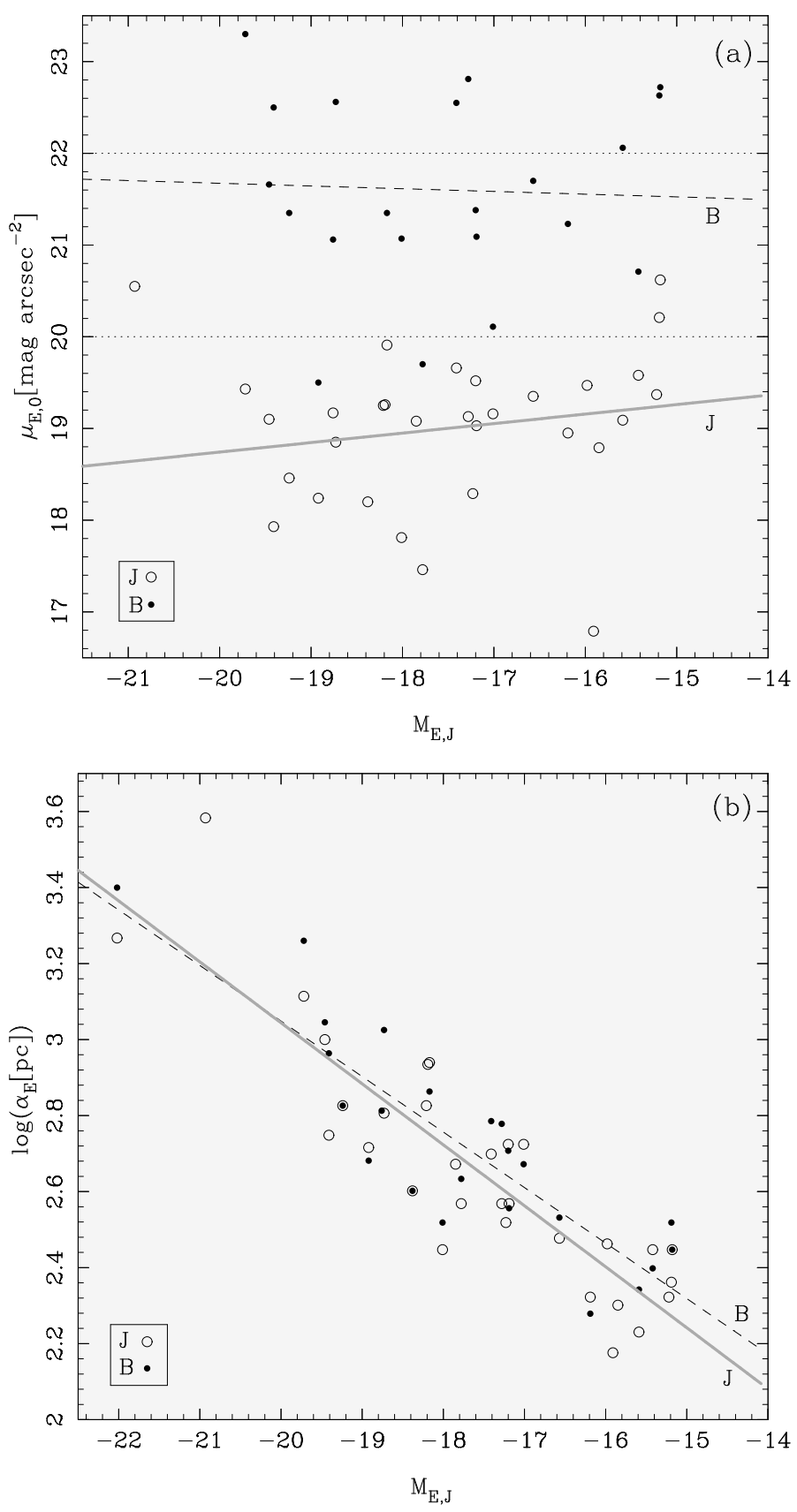

Fig. III.2. a) Extrapolated $B$ and $J$ band central surface brightnesses $\mu_{E, 0}$ and b) $B$ and $J$ band exponential scale lengths $\alpha_{E}$ of the LSB components of the sample galaxies, plotted against the absolute magnitude $\mathrm{M}_{\mathrm{E}}$ of the LSB component in the $J$ band. Straight gray and long-dashed black lines show respectively fits to the $J$ and $B$ data. Thin dotted lines in panel (a) indicate surface brightness levels of 20 and $22 \mathrm{mag} / \square^{\prime \prime}$ for orientation. 


$$
\begin{array}{ll}
<\alpha_{J} / \alpha_{B}>=0.90 & \sigma_{\mathrm{n}-1}=0.19 \\
<\alpha_{H} / \alpha_{B}>=0.86 & \sigma_{\mathrm{n}-1}=0.20 \\
<\alpha_{K} / \alpha_{B}>=0.85 & \sigma_{\mathrm{n}-1}=0.22
\end{array}
$$

The slight trend for the $\alpha_{E}$ in $J$ being by $\approx 10-15 \%$ smaller than in $B$, is mainly attributable to some galaxies with $\alpha_{E, \mathrm{NIR}}<0.8 \alpha_{E, B}$. In all of these cases, either the separation of the SF regions from the underlying old stellar host galaxy was problematic, or the $\mathrm{SBP}_{\mathrm{LSB}}$ shows a slightly concave shape, which is better fit by a Sérsic model with an exponent $\eta_{\mathrm{LSB}}>1$ (see N03a for details on profile fitting). For example, for the LSB host of the luminous $\left(M_{B}=-18.03\right)$ object Mkn 33, C03 report a Sérsic law with an exponent $\eta_{\mathrm{LSB}} \approx 2.9$ in $J$ and $\approx 1.4$ in $K$. Smaller $\alpha_{E}$ in the NIR are to be expected in such cases, since differing data qualities limit exponential fits to the LSB host to smaller radii in the NIR than in the optical.

The NIR-to-optical scale length ratio for Mkn 209, UM 462, Mkn 36 and Mkn 297 may also be slightly underestimated, either because of insufficient data depth, or due to very extended SF activity, which restrict the fit to the LSB component in the NIR to the low $S / N$ regime. Additionally, Mkn 297 is an intrinsically luminous BCG $\left(M_{B}=-21.0\right)$ galaxy merger candidate, rather than a BCD.

On the other hand, in two cases, Mkn 35 and Mkn 370, an asymmetric chain of SF regions, reaching out to the Holmberg radius, may have led to a slight overestimate of the scale length of the LSB component. This effect is probably more important in the optical than in the NIR, and may therefore lead to a reduced $\alpha_{\mathrm{NIR}} / \alpha_{B}$ ratio.

To summarize, from the present sample we find no solid evidence for the NIR scale length of the LSB component in BCDs being systematically smaller than in optical wavelengths. Excluding the above mentioned objects we deduce mean optical-NIR scale length ratios

$$
<\alpha_{J} / \alpha_{B}>\approx<\alpha_{H} / \alpha_{B}>\approx<\alpha_{K} / \alpha_{B}>\approx 1
$$

in support of the idea that the evolved LSB host of BCDs shows on average no appreciable color gradients, though individual exceptions can not be ruled out. In addition, as mentioned in N03a, the uncertainties of the $\alpha_{E}$ derived in the NIR for the current sample are $\gtrsim 10 \%$.

It is evident from Fig. III.2a that the sample BCDs generally have extrapolated central $J$ band surface brightnesses $\mu_{E, 0, J}<20 \mathrm{mag} / \operatorname{arcsec}^{2}$. Fainter $\mu_{E, 0}$ were derived for 3 objects, only, Mkn 178, Mkn 209 and the luminous BCG UM 448, where the $\mathrm{SBP}_{\mathrm{LSB}}$ could not be reliably constrained and exponential fits have been rather uncertain.

The approximative limit of $\mu_{E, 0} \lesssim 20 \mathrm{~J}$ mag/ $\square^{\prime \prime}$ corresponds to a $B$ surface brightness $\lesssim 22 \mathrm{mag} / \square^{\prime \prime}$, if a $B-J$ color $\lesssim 2.2$ is adopted. This is the approximative upper limit of this color, observed in the outer parts of the LSB components of the current sample (see N03a \& N03b, or compare the results in $\mathrm{C} 03$ to those presented in Cairós 2000).

An expected $B$ band limit of $\mu_{E, 0} \lesssim 22 \mathrm{mag} / \square^{\prime \prime}$ is roughly supported by the previous optical surface photometry as shown in Fig. III.2a. However, seven outlying galaxies 
display a $\mu_{E, 0}$ which is considerably fainter than this value. Two of these objects, Mkn 178 and Mkn 209, show faint $\mu_{E, 0}$ also in $J$ (see above). The remaining five outlying galaxies have, on the contrary, relatively bright $\mu_{E, 0}$ in the $J$ band, corresponding to implausibly red colors $\left(\mu_{E, 0, B}-\mu_{E, 0, J} \gtrsim 3.5\right)$ at the centers of their LSB hosts. It turns out that these objects are among the above discussed cases for which significantly larger $\alpha_{E}$ were derived in $B$ than in $J$. The large values of $\left(\mu_{E, 0, B}-\mu_{E, 0, J}\right)$ are therefore most likely attributable to an overestimation of $\alpha_{E}$ in $B$ : an error of $\alpha_{E}$ of $\sim 10-20 \%$ changes the corresponding $\mu_{E, 0}$ by $0.5-1 \mathrm{mag}$, due to the extrapolation from the region where the LSB is fitted $\left(R^{\star} \sim 2 \alpha_{E}\right)$ to $R^{\star}=0$.

\section{III.3.2 The dependence of the structural properties of the LSB host on its luminosity}

For the main class of evolved $\mathrm{iE} / \mathrm{nE}$ BCDs, the absolute magnitude of the LSB component $M_{\mathrm{E}}$ gives an approximative measure for the total stellar mass. The systematic difference of the LSB component of BCDs from quiescent gas-rich dwarf galaxies in the $\mu_{E, 0}-M_{\mathrm{E}}$ and $\alpha_{E}-M_{\mathrm{E}}$ parameter space (Fig. III.2a,b), detected first in P96b, may yield fundamental diagnostics for addressing the dynamical and evolutionary state of these systems. According to the results by P96b (see also Patterson \& Thuan 1996, Salzer \& Norton 1999 and Marlowe et al. 1997,1999), BCDs show a more compact structure of their stellar host galaxies, with $\alpha_{E}$ smaller by a factor of $\sim 2$ and $\mu_{E, 0}$ brighter by $\sim 1.5 \mathrm{mag}$, than dIs and dEs.

For the BCD sample analyzed here, we confirm the compact structure of their LSB hosts previously found in the $B$ band. This rules out that the compact optical structure inferred for the underlying LSB component of BCDs is an artifact of, e.g., contamination by residual SF light in optical wavelengths. On the contrary, the results discussed in Section III.3.1 suggest that residual starburst emission may lead to the determination of a slightly larger $\alpha_{E}$ and $\mu_{E, 0}$ from optical data, and moderate thereby the structural dichotomy between BCDs and other dwarf galaxies.

Linear fits to the BCD data (Fig. III.2b) yield the relations

$$
\begin{aligned}
& \log \alpha_{\mathrm{E}, J}=-(0.16 \pm 0.02) M_{\mathrm{E}, J}-(0.17 \pm 0.28) \\
& \log \alpha_{\mathrm{E}, B}=-(0.15 \pm 0.02) M_{\mathrm{E}, J}+(0.13 \pm 0.31)
\end{aligned}
$$

with $\alpha_{E}$ and $\mu_{E, 0}$ in units of pc and mag, respectively. As expected from the equality between $\alpha_{J}$ and $\alpha_{B}$ (Sect. III.3.1), the slopes in Eqs. III.9 and III.10 are identical. The r.h.s. constants in both equations can be taken to be zero, given the large uncertainty in the extrapolation of the slope to $M_{\mathrm{E}}=0$.

If the above relations, Eqs. (III.9) and (III.10), are derived for our sample using $M_{\mathrm{E}}$ in the $B$ band as taken from the literature (see Table III.1), we obtain

$$
\begin{aligned}
& \log \alpha_{\mathrm{E}, J}=-(0.15 \pm 0.02) M_{\mathrm{E}, B}-(0.32 \pm 0.36) \\
& \log \alpha_{\mathrm{E}, B}=-(0.15 \pm 0.03) M_{\mathrm{E}, B}+(0.36 \pm 0.45)
\end{aligned}
$$

in agreement with the relations inferred before. 
Note that a possible non-detection of a centrally flattening $\mathrm{SBP}_{\mathrm{LSB}}$ from optical data, and consequently an overestimate of the LSB luminosity for part of the sample BCDs (see discussion in N03a and Sect. III.4), will not notably change the slope between $\alpha$ and $M_{\mathrm{E}}$ in Eqs. (III.9) and (III.10). It would merely result in a minor shift in the axis intercepts of the linear fits (Fig. 2).

From the study of a first, small sample of BCDs, P96b found no clear statistical evidence that the trend for a decreasing central surface brightness $\mu_{E, 0}$ with fainter LSB magnitude continues for $M_{\mathrm{E}}>-16 B$ mag. However, a larger data set which was recently compiled (e.g. Papaderos et al. 2002; see their Fig. 1, or Fig. III.2a, this paper) shows that a tendency for fainter $\mu_{E, 0}$ with fainter $M_{\mathrm{E}}$ is still observable towards the faint end of the dwarf galaxy sequence, although with considerable scatter. Nonweighted linear fits to the available data yield a weak trend between $\mu_{E, 0}$ and $M_{\mathrm{E}}$ of the form

$$
\begin{gathered}
\mu_{0, J}=(0.10 \pm 0.09) M_{\mathrm{E}, J}+(20.8 \pm 1.6) \\
\mu_{0, B}=(0.20 \pm 0.15) M_{\mathrm{E}, B}+(24.6 \pm 2.4) .
\end{gathered}
$$

Additional support for a dependence of $\mu_{E, 0}$ on $M_{\mathrm{E}}$ in both optical and NIR wavelengths can be derived from solving Eq. (III.4) for $\log \left(\alpha_{\mathrm{E}}\right)$ :

$$
\log \left(\alpha_{\mathrm{E}}[\mathrm{pc}]\right)=-0.2 M_{\mathrm{E}}+0.2 \mu_{\mathrm{E}, 0}-4.714 \text {. }
$$

If $\mu_{E, 0}$ was on average independent of $M_{\mathrm{E}}$, the $\log \left(\alpha_{\mathrm{E}}\right)-M_{\mathrm{E}}$ relation should show a slope of -0.2 , significantly steeper than the one empirically found $(\approx-0.15 \pm 0.02$; Eqs. III.9-III.12). Combining Eqs. (III.4), (III.9) and (III.12), we can independently derive a relation between $\mu_{E, 0}$ and $M_{\mathrm{E}}$ as:

$$
\begin{gathered}
\mu_{0, J}=(0.20 \pm 0.10) M_{\mathrm{E}, J}+(22.7 \pm 1.4) \\
\mu_{0, B}=(0.25 \pm 0.15) M_{\mathrm{E}, B}+(25.4 \pm 2.3) .
\end{gathered}
$$

Both latter relations are consistent with the fit results Eqs. (III.13) and (III.14) within their uncertainties.

\section{III.4 Inwards-flattening exponential (type V) $\mathrm{SBP}_{\mathrm{LSB}}$}

Inwards-flattening exponential $\mathrm{SBP}_{\mathrm{LSB}}$, classified "type $\mathrm{V}$ " for dwarf galaxies by Binggeli \& Cameron (1991), were first detected in BCDs by P96a (see also references in Guseva et al. 2001). However, clear observational evidence for a frequent occurrence of such $\mathrm{SBP}_{\mathrm{LSB}}$ in BCDs was first obtained from deep NIR surface photometry studies in N03a. Next we address the question of the frequency of type $\mathrm{V}$ profiles in BCDs and undergo steps towards their systematization. Our discussion is based on the $J$ band data by N03a and N03b, that has been processed and analyzed in a uniform manner (see Sect. III.2). Out of those 24 objects, 22 are BCDs (classified iE/nE/iI/iI,C in Tab. III.1). 
Following the discussion of our previous paper N03a, we use the modified exponen-

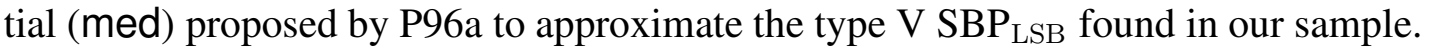

$$
I\left(R^{\star}\right)=I_{\mathrm{E}, 0} \exp \left(-\frac{R^{\star}}{\alpha}\right)\left\{1-q \exp \left[-P_{3}\left(R^{\star}\right)\right]\right\}
$$

with

$$
P_{3}\left(R^{\star}\right)=\left(\frac{R^{\star}}{b \cdot \alpha}\right)^{3}+\left(\frac{R^{\star}}{\alpha} \cdot \frac{1-q}{q}\right) .
$$

As was already stated in N03a, the precise shape and physical origin of type V SBPs in spheroidal dwarf galaxies is to date not studied. Therefore the choice of Eq. (III.18) does not reflect a preference to a physical model of the mass distribution or dynamics of the stellar LSB component. Instead, this function has been worked out by P96a as a tool which allows to empirically quantify the main structural features of type $\mathrm{V} \mathrm{SBP}_{\mathrm{LSB}}$ in such galaxies. In addition to $I_{\mathrm{E}, 0}$ and $\alpha_{\mathrm{E}}$, the distribution given by Eq. III.18 depends on the cutoff radius $b \alpha_{\mathrm{E}}$ inwards of which the exponential profile levels off, and on the relative intensity depression $q=\Delta I / I_{\mathrm{E}, 0}(<1)$ with respect to the central intensity $I_{\mathrm{E}, 0}$ as predicted by extrapolation of the outer exponential slope to $R^{*}=0^{\prime \prime}$. Equation (III.18) approximates the observed $\mathrm{SBP}_{\mathrm{LSB}}$ by a single smooth function, showing beyond the cutoff-radius $b \alpha$ a perfectly exponential fall-off with the scale length $\alpha_{\mathrm{E}}$. By this fact, the med reproduces adequately the exponential intensity decrease at large $R^{*}$, which is generally derived in the outskirts of the LSB component. Equation (III.18) is an extension rather than a revision of the commonly used exponential law. In particular, the med does not alter the results of previous studies of the $\mu_{\mathrm{E}, 0}-\alpha_{\mathrm{E}}$ parameter space of dwarf galaxies. This is not the case for Sérsic models, which, although fitting satisfactorily type $\mathrm{V}$ profiles (see, e.g., N03a and references therein), yield a scale parameter that is not identical to that of an exponential model. Additionally, for a reasonable combination of $b$ and $q$, the intensity distribution Eq. (III.18) yields a monotonous and finite intrinsic luminosity density within the LSB component, contrary to Sérsic models with $\eta_{\mathrm{LSB}}<0.5$ (P96a).

This approach is similar to e.g. the one by Näslund \& Jörsäter (1997) and Pohlen et al. (2002a, and references therein), who used two differing exponentials inside and outside a "break radius" $R_{\mathrm{br}}$ to fit the SBPs of low-surface-brightness spiral galaxies. This kind of double-exponential distributions, with an extended transition region around a cutoff radius, can alternatively be used to approximate the type $\mathrm{V} \mathrm{SBP}_{\mathrm{LSB}}$ profiles observed in N03a and N03b.

\section{III.4.1 Frequency of type V SBP ${ }_{\text {LSB }}$ and relation to the BCD morphology}

The smaller BCD sample studied in N03a, although overrepresenting irregular morphological types, had already pointed to the presence of type $\mathrm{V}$ profiles in the LSB component of more than one half of BCDs. This high fraction is confirmed on inspection of our extended BCD sample (Table III.1): in 12 of 22 BCDs (i.e. $55 \%$ with a

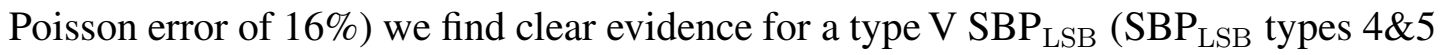



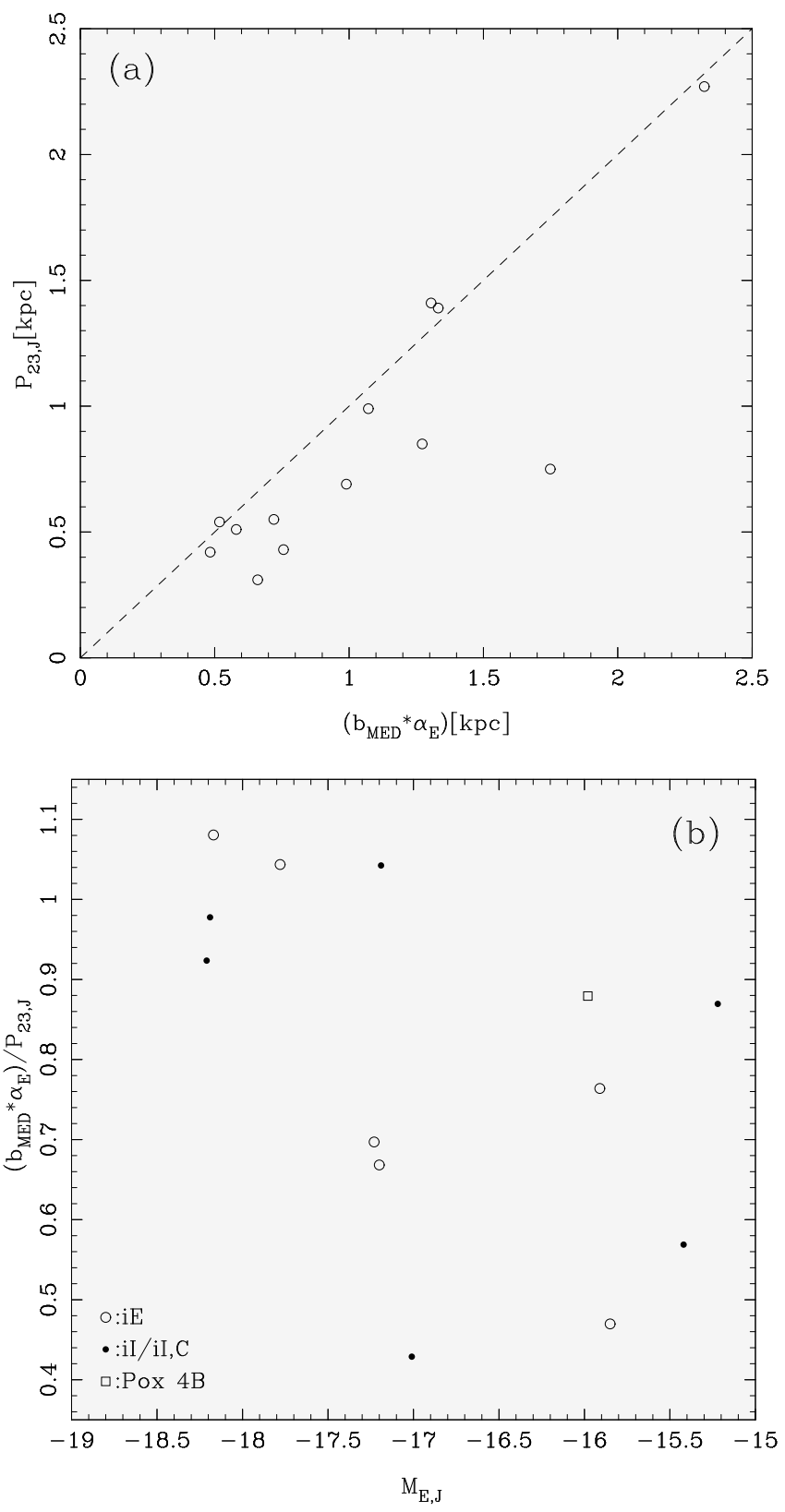

Fig. III.3. a) Radius $P_{23}$ of the starburst component, determined at the isophotal level of 23 $J \mathrm{mag} / \square^{\prime \prime}$, vs. the cutoff radius $b \alpha_{\mathrm{E}}$ of the underlying LSB host galaxy for all sample BCDs with a type V LSB profile (type $4 \& 5$ in Tab. III.1). The long-dashed line shows equality of both radii. b) Ratio of the cutoff radius $b \alpha_{E}$ to the starburst radius $P_{23}$ as a function of $M_{\mathrm{E}}$. Note the trend of the cutoff-radius $b \alpha_{E}$ to approach the starburst radius $P_{23}$ for the brightest sample galaxies. 
in Tab. III.1, see Sect. III.2). Four other BCDs are type V candidates ( $\mathrm{SBP}_{\mathrm{LSB}}$ type 3) and two show no reliably classifiable $\mathrm{SBP}_{\mathrm{LSB}}$ (denoted type 2). Only in four sample BCDs we observe a perfectly exponential $\mathrm{SBP}_{\mathrm{LSB}}$ (type 1).

Perhaps, type $\mathrm{V}$ profiles are not equally frequent among different morphological classes of BCDs (see Table III.1). They appear very common among irregular iI/iI,C BCDs (observed in 9 of the 10 objects). Out of the $13 \mathrm{iE} / \mathrm{nE}$ sample BCDs, only in $4 \mathrm{a}$ type $\mathrm{V}$ profile is unambiguously detected. Four other iE/nE BCDs (He 2-10, Mkn 324, Tol 3, Mkn 996) show no deviations from the exponential law throughout their LSB intensity regime. From Fig. III.5b is evident that these galaxies have bright $\left(M_{\mathrm{E}} \lesssim-18\right)$ LSB hosts. All show, in addition, a strong, centrally concentrated starburst component (high concentration indices $C I$ ), as expected from the relation between $M_{\mathrm{E}}$ and $C I$ (see Sect. III.5.2.4).

\section{III.4.1.1 Completeness}

As was pointed out in N03a, NIR data moderate, yet not eliminate problems in the direct study of the LSB host galaxy in its central part. Extended starburst emission

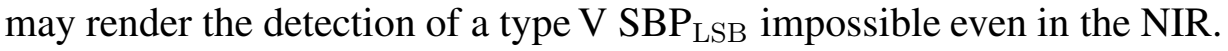

A comparison of the radius inside of which the line-of-sight intensity of the starburst becomes significant, here approximated by the plateau radius $P_{23}$ (cf. paper I and P96a,b), to the empirical cutoff-radius $b \alpha_{\mathrm{E}}$ within which a central flattening occurs, is shown in Fig. III.3a. All sample BCDs with a type $\mathrm{V}$ profile are located in the region where $P_{23, J} \lesssim b \alpha_{\mathrm{E}}$. For those systems we obtain a mean starburst-to-SBP $\mathrm{LSB}_{\text {cutoff }}$ ratio of

$$
\left\langle\frac{P_{23, J}}{b \cdot \alpha_{\mathrm{E}}}\right\rangle=0.80 \pm 0.06, \sigma_{n-1}=0.23
$$

with a maximal value of $\left(P_{23, J} / b \alpha_{\mathrm{E}}\right)_{\max }=1$.1. Figure III.3a therefore suggests that type $\mathrm{V} \mathrm{SBP}_{\mathrm{LSB}}$ profiles typically escape detection whenever their cutoff radius $b \alpha_{\mathrm{E}}$ is significantly smaller than $P_{23, J}$. Consequently, a weak central flattening cannot be excluded even for those BCDs which show a perfectly exponential intensity decrease in their LSB periphery (type 1 in Table III.1).

This is supported by the evidence of Fig. III.3b, which suggests that the cutoff radii approach the starburst radii for increasing LSB luminosities. As stated in the last subsection, the $4 \mathrm{BCDs}$ with apparently purely exponential $\mathrm{SBP}_{\mathrm{LSB}}$ have throughout luminous LSB hosts, so that their cutoff radii may be considerably smaller than their starburst radii, rendering a central flattening undetectable. The differences to a purely exponential $\mathrm{SBP}_{\mathrm{LSB}}$ model would in these cases be small, other than for the large $b, q$ we derive for most of the type $\mathrm{V} \mathrm{SBP} \mathrm{LSB}_{\mathrm{LB}}$.

From such considerations, we hypothesize that the true fraction of BCDs with a type $\mathrm{V} \mathrm{SBP}_{\mathrm{LSB}}$ may be much larger than the percentage of $55 \%$ deduced here. 


\section{III.4.1.2 Relations between the flattening parameters and integral properties of the LSB host}

It is worthwhile to study whether the cutoff-radius and central intensity depression of a type $\mathrm{V}$ profile correlate with other properties of the LSB host galaxy (e.g., absolute magnitude). Approximating a type V profile with Eq. (III.18) we find that, except for the tendency shown in Fig. III.3b, neither $b$ or $b \alpha_{E}$, nor $q$ show significant correlations with $\mu_{E, 0}, \alpha_{E}$ or $M_{\mathrm{E}}$. Also the frequency of the type $\mathrm{V} \mathrm{SBP}_{\mathrm{LSB}}$ we find does not exhibit any relation to $M_{\mathrm{E}}$, apart from the possible bias against the most luminous LSB hosts discussed in the previous subsection. Albeit the small sample size, this suggests that mechanisms leading to the development of type $\mathrm{V} \mathrm{SBP}_{\mathrm{LSB}}$ in $\mathrm{BCDs}$ are not operating exclusively for a certain morphological type or luminosity span of the LSB component.

A close relation is found between the flattening parameters $q$ and $b$ themselves, shown in Fig. III.4 for the 12 secure type V BCDs and the star-forming dwarf galaxy Pox 4B. P96a proposed that a tentative ratio $b \simeq 3 q$ is adequate for type $\mathrm{V}$ profiles with a comparatively small central depression (i.e., for $q \lesssim 0.4$ ). They conjectured, however, that $b / q$, as well as $q$ itself may depend on $M_{\mathrm{E}}$.

In the range of stronger flattening parameters found for the present sample, i.e. for $q \gtrsim 0.6$, the solutions of $(b, q)$ can be fitted by a function of the form:

$$
q=1-\frac{0.76}{b^{2}}
$$

A maximal $q$ for a given $b$ is to be expected, since a strong central depression implies, for too small cutoff radii, implausible radial distributions of the stellar surface density (cf. P96a, Fig. 13). However, the derived solutions of $(b, q)$ do not seem to delineate a loosely defined parameter space to which Eq. (III.21) gives an upper envelope, but apparently follow a tight relation. Of course, a direct determination of the $b / q$ ratio is virtually impossible in the range of small cutoff-radii (for $b \lesssim 1.5 \alpha_{\mathrm{E}}$ ) where starburst emission typically dominates. The outlying points are Tol 1400-411, NGC 6789 and I $\mathrm{Zw} 115$, galaxies for which the $q$ might be slightly underestimated. Interestingly, the degree of the central flattening of the $\mathrm{SBP}_{\mathrm{LSB}}$ shows a trend to increase towards higher starburst strengths (Fig. III.4b\&c). This may, again, be partly due to difficulties in detecting a small flattening in the presence of an extended starburst. It is furthermore noteworthy that a slight underestimation in $q$ by the profile decomposition results in an overestimation of the starburst luminosity, and vice versa.

\section{III.5 Relations between the structure of the LSB host and the properties of the starburst}

\section{III.5.1 Luminosity contribution by the starburst}

The luminosity ratio of the starburst component $L_{\mathrm{SB}}$ and the underlying stellar LSB host $L_{\mathrm{E}}$ is known from optical studies (Drinkwater \& Hardy 1991, P96b, Salzer \& 

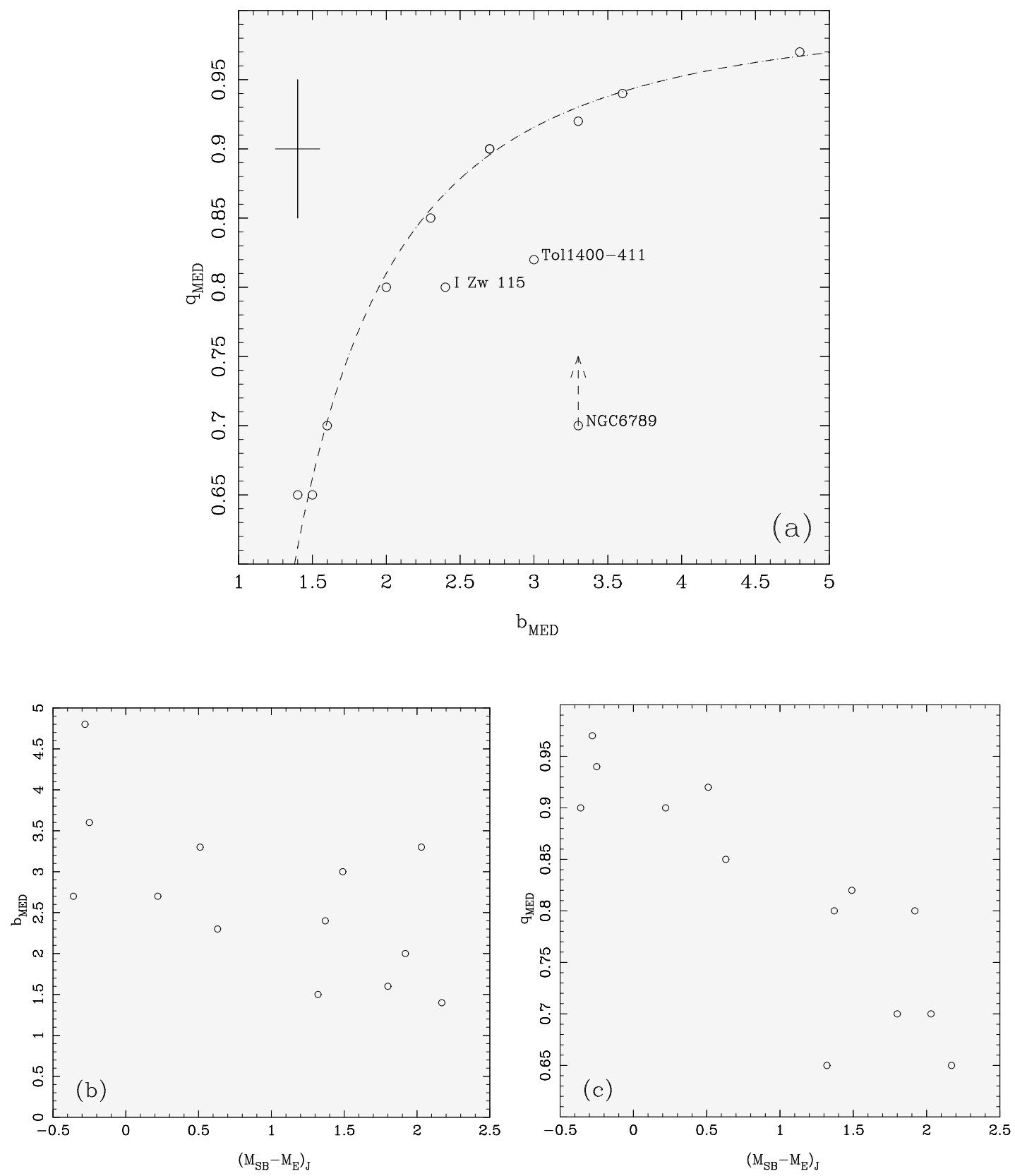

Fig. III.4. a Flattening parameters $b$ and $q$ (see Eq. III.18) of the confirmed type V $\mathrm{SBP}_{\mathrm{LSB}}$ (classified as 4 and 5 in col. 3 of Table III.1). The long-dashed curve shows a fit (Eq. III.21) to the upper envelope of the data, excluding NGC 6789, Tol 1400-411, and I Zw 115, for which $q$ may be more strongly underestimated than for the other objects. The cross in the upper left corner gives typical uncertainties of $b$ and $q . \mathbf{b , c} b$ and $q$ as a function of the relative burst strength, represented by the difference of the absolute magnitudes of the starburst $\left(M_{\mathrm{SB}}\right)$ and the stellar host $\left(M_{\mathrm{E}}\right)$. 
Norton 1999) to show a considerable scatter. The spread of this ratio certainly originates to a large extent from the fact that the optical/NIR luminosity of a starburst depends strongly on the burst age. Nevertheless, the average $\left\langle L_{\mathrm{SB}} / L_{\mathrm{E}}\right\rangle$ ratio is useful for estimating the photometric fading which a BCD can on average undergo after the termination of its SF activity.

From the present sample, we derive average values, errors about the mean and sample standard deviations $\sigma_{n-1}$ in the $B$ and $J$ bands:

$$
\begin{aligned}
\left\langle L_{\mathrm{SB}} / L_{\mathrm{E}}\right\rangle_{B} & =1.75 \pm 0.30, & & \sigma_{n-1}=1.28 \\
\left\langle L_{\mathrm{SB}} / L_{\mathrm{E}}\right\rangle_{J} & =0.57 \pm 0.10, & & \sigma_{n-1}=0.52
\end{aligned}
$$

from 19 and 32 sample points in the $B$ and $J$ bands, respectively ${ }^{1}$ with a $B$ band median value of 1.57. These values correspond to starburst light fractions of the total light of the galaxy of $\approx 0.4 \pm 0.1$ in $J$ and $\approx 0.6 \pm 0.1$ in $B$. None of these luminosity ratios shows a significant correlation with the absolute magnitude $M_{\mathrm{E}}$ of the host galaxy (cf. Fig. III.8a).

One should be cautious in making comparisons between the luminosity ratio $\left\langle L_{\mathrm{SB}} / L_{\mathrm{E}}\right\rangle$ as derived in optical and NIR wavelengths. As remarked in N03a, type V $\mathrm{SBP}_{\mathrm{LSB}}$ remain in most cases undetected in the $B$ band. The decomposition of those SBPs was mostly performed by means of an exponential fit to the $\mathrm{SBP}_{\mathrm{LSB}}$, which leads to an overestimation of $L_{\mathrm{E}}$ and, correspondingly, to an underestimation of $L_{\mathrm{SB}}$.

Those objects for which both in the $B$ and $J$ bands a clearly exponential $\mathrm{SBP}_{\mathrm{LSB}}$ was found can be considered to be adequately decomposed by an exponential in the $B$ band. These show an average $\left\langle L_{\mathrm{SB}} / L_{\mathrm{tot}}\right\rangle_{B} \approx 0.6$. For those galaxies which were approximated by an exponential in the $B$ band, but were in the $J$ band unambiguously

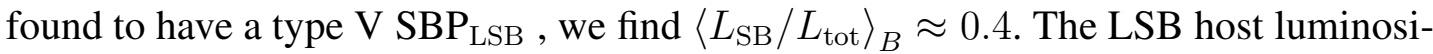
ties were in the latter cases overestimated by an average factor of $\sim 1.5$. This value corresponds to an average underestimation of the starburst flux by a factor of $\sim 2 / 3$, or to an underestimation of the averaged $\left\langle L_{\mathrm{SB}} / L_{\mathrm{E}}\right\rangle$ ratio by a factor of $\sim 0.5$. Since, however, few data points ( 5 in either of the latter subsamples) entered in this estimation, and the intrinsic scatter of the $L_{\mathrm{SB}} / L_{\mathrm{E}}$ ratios is large as mentioned above, these factors should be considered indicative only.

\section{III.5.2 A tentative link between the structure of the stellar host and the occurrence and extent of burst-like star formation in typical BCDs}

Previous work has established that SF in BCDs proceeds at a much higher rate than in dIs, in a burst-like mode which in many cases comes along with formation of Super Stellar Clusters (SSCs, e.g. O'Connell et al. 1994). By contrast, dIs present a more extended, low-level mode of SF (see, e.g., Patterson \& Thuan 1996) which results merely in a modest luminosity enhancement. These fundamentally different modes of star formation take place in systems with comparable mass and gas content, but strikingly different mass distributions. BCDs show a compact structure and larger mass

\footnotetext{
${ }^{1}$ The luminous BCG Mkn 297, a likely post-merger, was excluded.
} 
concentration with respect to both, their stellar and gaseous component, most notably, a by a factor of $\sim 10$ higher central mass density than dIs (P96b, Papaderos \& Fricke 1998, van Zee et al. 1998,2001). P96b and Papaderos (1998) found that the amplitude and fractional area of the starburst component in a BCD are not fortuitous, but apparently related to the structural properties and luminosity of the underlying old LSB host galaxy. A similar behaviour has been recently reported for dIs (Hunter et al. 1998) and Spiral Galaxies (Ryder \& Dopita 1994). In either class of galaxies, the surface densities of the old stars and of the SF activity were found to correlate. Interestingly, the surface densities of the $\mathrm{HI}$ and the star-formation rates per unit area in dIs do not display a comparably tight correlation (Hunter et al. 1998).

Such lines of evidence suggest that the gravitational potential formed by the higher mass-to-light ratio LSB host either provides by itself, or closely traces, the conditions needed for burst-like SF activity in the case of BCDs, or for a more continuous lowlevel SF activity in the case of dIs. Since the observed stellar surface densities are closely related to the mean volume density, one conceivable hypothesis is that there is a certain stellar mass density in the underlying stellar LSB background favouring burst-like SF activity.

\section{III.5.2.1 Is the onset of starburst activity related to the stellar mass density of the LSB host?}

To test the hypothesis of a critical stellar density related to burst-like star formation, we estimate here the stellar luminosity density $l_{\mathrm{E}}$ of the underlying LSB host at the characteristic plateau radius $P_{23}$. The plateau component includes the light not only from the very young stellar population but also from a moderately evolved population with an age of a few $\sim 10^{7}$ and $\sim 10^{8} \mathrm{yr}$ (cf. P96a). $P_{23}$ encloses therefore the average volume within a BCD which has been susceptible to SF activity over a longer timespan.

Since the $M / L$ may be regarded roughly constant within the LSB host, as indicated by the approximately constant color profiles of this component (P96a; Cairós et al. 2001a; N03a,b; C03), the luminosity density $l_{\mathrm{E}}$ can be directly transformed to a stellar mass density $\rho_{\mathrm{E}}$. To this end we assume a $M / L_{J}$ of $\approx 2$ which is typical for a few Gyr old stellar population with subsolar metallicity (Anders 2003, priv. comm., Schulz et al. 2002).

The derivation of $l_{\mathrm{E}}\left(R^{\star}\right)$ from the projected radial intensity distribution requires high S/N data, and is subject to uncertainties connected with the assumptions that are made about the 3D distribution of stellar mass in the LSB component. We utilize here the approximations to the luminosity density of an exponential intensity profile given in P96a. These authors adopted spherical symmetry, most likely a better approximation to the 3-dimensional stellar mass distribution of BCDs than a disk geometry (cf. e.g. Sung et al. 1998, Koprolin 2002).

P96a adopted a med with a small central intensity flattening of $(b, q)=(0.3,0.1)$, to circumvent the problem of an indefinite central luminosity density occurring for a purely exponential profile. The intrinsic luminosity density $l_{\mathrm{E}}\left(R^{\star}\right)$ in $L_{\odot} \mathrm{pc}^{-3}$ of such an intensity profile can be approximated in the radius range $2 \alpha_{\mathrm{E}} \leq R^{\star} \leq 7 \alpha_{\mathrm{E}}$ as 


$$
l_{\mathrm{E}}\left(\frac{R^{\star}}{\alpha_{\mathrm{E}}}\right)=\frac{4 I_{\mathrm{E}, 0}}{\alpha_{\mathrm{E}}} \exp \left(-\frac{R^{\star}}{0.9 \alpha_{\mathrm{E}}}\right) .
$$

(see Fig. 14 in P96a). The central surface brightness $\mu_{E, 0}$ of such a profile reads as

$$
I_{\mathrm{E}, 0}\left[L_{\odot} \mathrm{pc}^{-2}\right]=4.25 \times 10^{8-0.4\left(\mu_{\mathrm{E}, 0}-M_{\odot}^{\mathrm{abs}}\right)}
$$

with an absolute solar $J$ magnitude $M_{\odot}^{\text {abs }}=3.70$ (Worthey et al. 1994). For $R^{\star}<2 \alpha_{\mathrm{E}}$, the $l_{\mathrm{E}}\left(R^{\star}\right)$ will be underestimated by only $\lesssim 0.1 \mathrm{dex}$ if $\alpha_{\mathrm{E}} \leq R^{\star} \leq 2 \alpha_{\mathrm{E}}$ and $(b, q)=(0.3,0.1)$, and stronger for smaller $(b, q)$. However, the $(b, q)$ we typically derived are considerably larger than $(0.3,0.1)$, corresponding to an overestimation of $l_{\mathrm{E}}\left(R^{\star}\right)$ by Eq. (III.24), which counteracts the underestimation $l_{\mathrm{E}}$ of at small $R^{\star}$ by the same equation.

An attempt to determine $l_{\mathrm{E}}\left(R^{\star}\right)$ more precisely through a numerical deprojection of each $\mathrm{SBP}_{\mathrm{LSB}}$ is hardly meaningful, in view of the incompleteness of the present data sample and the uncertainties in fitting the $\mathrm{SBP}_{\mathrm{LSB}}$. Such uncertainties are aggravated by intrinsic uncertainties connected with the 3-dimensional object geometry (for instance, inclination of an oblate spheroid), radiative transfer, and the precise slope of a med at small radii (generally for $R^{\star} \lesssim P_{23}$ ). Moreover, we are not primarily interested in the actual density distribution of the LSB component at small radii, but rather in an empirical estimate of the minimum underlying stellar density at the radius $P_{23}$.

We subsume the above mentioned uncertainties, which cannot be eliminated further, in a deprojection deviation function $C_{1}$ (Eq. III.26), which describes for each object the ratio of the true $l_{\mathrm{E}}\left(R^{\star} / \alpha_{\mathrm{E}}\right)$ to the one derived from Eq. (III.24) (the denominator in Eq. III.26); $g$ accounts for the intrinsic geometry, $\mathrm{SBP}_{\mathrm{LSB}}$ shape and inclination of each BCD; $g_{a}$ in the denominator denotes the configuration adopted in Eq. (III.24), i.e. spherical symmetry and a pure med $\mathrm{SBP}_{\mathrm{LSB}}$.

$$
C_{1, \mathrm{obj}}\left(\frac{R^{\star}}{\alpha_{\mathrm{E}}}\right)=\frac{l\left(\frac{R^{\star}}{\alpha_{\mathrm{E}}},\left(\mu_{\mathrm{E}, 0}, \alpha_{\mathrm{E}}, b, q, g\right)_{\mathrm{obj}}\right)}{l\left(\frac{R^{\star}}{\alpha_{\mathrm{E}}},\left(\mu_{\mathrm{E}, 0}, \alpha_{\mathrm{E}}\right)_{\mathrm{obj}}, 0.3,0.1, g_{a}\right)}
$$

We restrict our discussion to 'typical' $\mathrm{iE} / \mathrm{nE}$ BCDs from the samples of N03a and N03b (Table III.1), and the moderately irregular objects Mkn 5, UM 461 and I Zw 115. Mkn 209 is excluded due to large uncertainties in its $\mathrm{SBP}_{\mathrm{LSB}}$ decomposition. Displaying relatively regular, elliptical LSB hosts, these objects are most likely to approximately match the assumption of spheroidal geometry. This is less obvious for the extreme iI,C objects (Tol 1214-277, Tol 65, Tol 1400-411, Mkn 1329) which may systematically differ from $\mathrm{iE} / \mathrm{nE}$ BCDs with respect to their 3-dimensional properties and evolutionary status (Noeske et al. 2000, Papaderos et al. 1999, Fricke et al. 2001). In addition, galaxies with known or likely close companions were removed from the sample, since interactions may have altered conditions for the onset of starburst activity on a global scale (Pox 4, see Méndez \& Esteban 1999; IC 4662, see Hidalgo-Gámez et al. 2002; NGC 5058, Tifft 1982).

Figure III.5a shows the $J$ stellar luminosity densities $l_{\mathrm{E}}\left(P_{23} / \alpha_{\mathrm{E}}\right)$ as estimated from Eq. (III.24), i.e. the true values, multiplied by the unknown deprojection deviation 

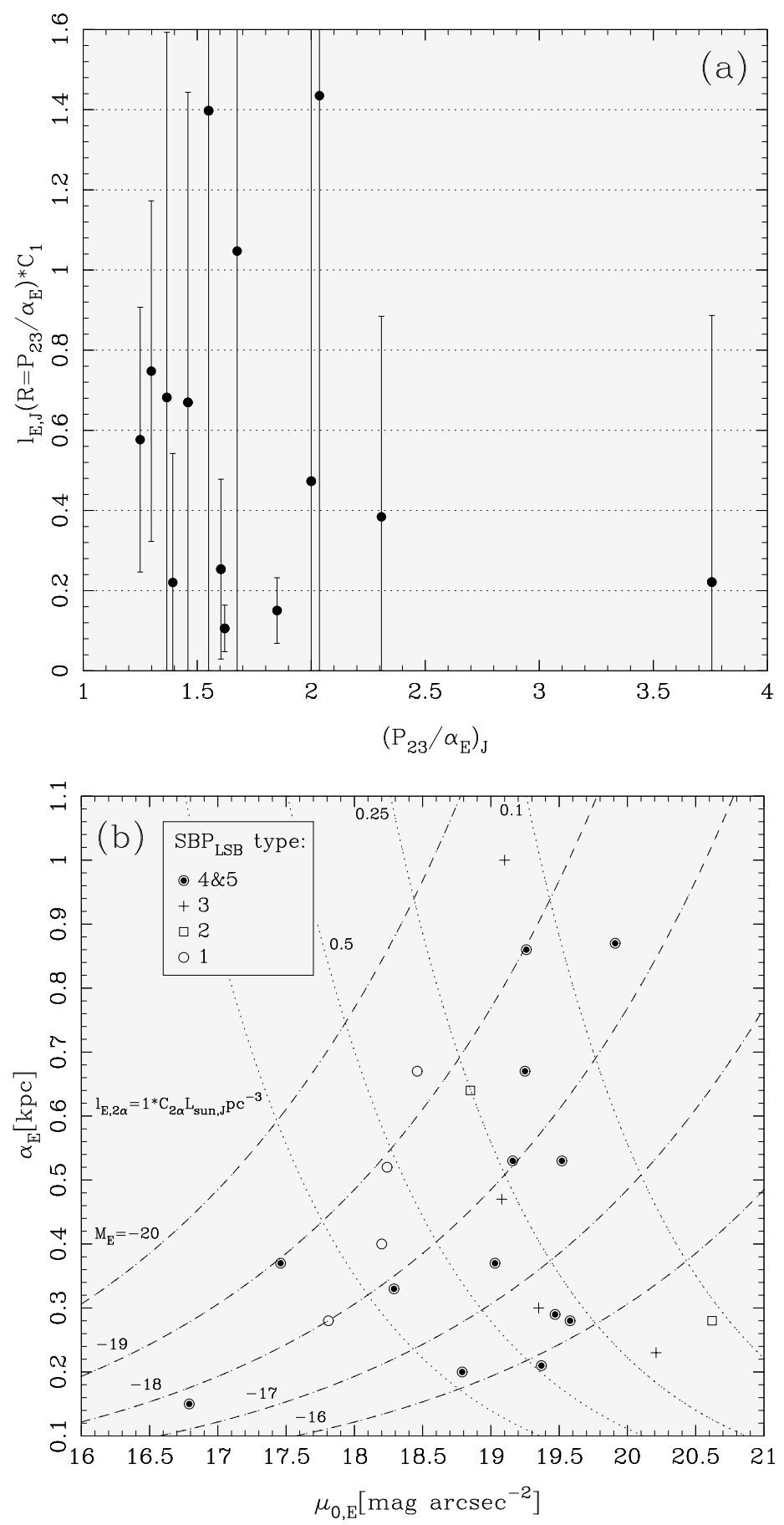

Fig. III.5. a) $J$ band stellar luminosity density $l_{\mathrm{E}}$ of the underlying LSB host galaxy at the starburst radius $P_{23}$, vs $P_{23}$ normalized to the scale length $\alpha_{E}$. $l_{\mathrm{E}}$ has been estimated from Eq. (III.24). The luminous galaxy UM 448 is excluded from the diagram. b) $\mu_{\mathrm{E}, 0}-\alpha_{\mathrm{E}}$ parameter space in the $J$ band for the sample from N03a and N03b. The symbols refer to the different subdivisions of the available sample with regard to the $\mathrm{SBP}_{\mathrm{LSB}}$ shape, as indicated in col. 3 of Table III.1. Long-dashed curves show isoluminosity contours of the LSB hosts. Dotted lines indicate contours of equal stellar density for the LSB hosts at $R^{\star}=2 \alpha\left(l_{\mathrm{E}, 2 \alpha}\right)$, calculated for a med with $(b, q)=(0.3,0.1)$ and $l_{\mathrm{E}, 2 \alpha}$ of $1,0.5,0.25$ and $0.1 L_{\odot, J} \mathrm{pc}^{-3}$. 
function $C_{1}$ for each data point (Eq. III.26). Since $C_{1}$ is a function of $\left(P_{23} / \alpha_{\mathrm{E}}\right)$, we indicate the latter value on the abscissa. It is evident that $l_{\mathrm{E}}\left(P_{23} / \alpha_{\mathrm{E}}\right)$ shows no systematic dependence on $\left(P_{23} / \alpha_{\mathrm{E}}\right)$. Errors of $l_{\mathrm{E}}$ are calculated adopting the typical measurement uncertainties of $10 \%, 25 \%$ and 0.1 mag for $\alpha_{E}, P_{23}$ and $\mu_{E, 0}$, respectively ${ }^{2}$. The uncertainties grow rapidly for larger $l_{\mathrm{E}}$, i.e. smaller $R^{\star}$, due to the exponential dependence of $l_{\mathrm{E}}$ on $\left(-R^{\star}\right)$ in Eq. (III.24).

From the observed range of $l_{\mathrm{E}}\left(P_{23} / \alpha_{\mathrm{E}}\right)$, we estimate a lower limit of $l_{\mathrm{E}}\left(P_{23} / \alpha_{\mathrm{E}}\right)$ of $\sim 0.2 \pm 0.1 L_{\odot, J} \mathrm{pc}^{-3}$, taking into account that at $P_{23}<2 \alpha_{E}$ the deprojection deviation $C_{1}$ may significantly differ from unity, and therefore assuming that some points are slightly underestimated $\left(C_{1} \cdot l_{\mathrm{E}}\left(P_{23} / \alpha_{\mathrm{E}}\right)<0.2 L_{\odot, J} \mathrm{pc}^{-3}\right)$. We therefore restrict our discussion to the lower limit of $l_{\mathrm{E}}$ estimated from Fig. III.5, and raise the hypothesis of a minimum stellar luminosity density of $l_{\text {crit }} \sim 0.2 \pm 0.1 L_{\odot, J} \mathrm{pc}^{-3}$, corresponding to an underlying stellar mass density of $\rho_{\text {crit }} \sim 0.4 \pm 0.2 \mathrm{M}_{\odot} \mathrm{pc}^{-3}$, below which burst-like SF is unlikely to occur. In the following we test whether such a strongly simplifying assumption is consistent with the observations.

\section{III.5.2.2 The $\alpha_{E-\mu_{E, 0}}$ vs. $M_{\mathrm{E}}$ relation}

Here, we will use the structure-luminosity relations found for different dwarf galaxies, $\alpha_{E}$ vs. $M_{\mathrm{E}}$ and $\mu_{E, 0}$ vs. $M_{\mathrm{E}}$ (Sect. III.3.2), to further assess the hypothesis that stellar density may act as a regulating agent of burst-like SF activity. From Eq. (III.4), it is apparent that the $\alpha_{E}-M_{\mathrm{E}}$ and $\mu_{E, 0}-M_{\mathrm{E}}$ relations can be understood as transformations of the $\mu_{E, 0}-\alpha_{E}$ parameter space populated by BCDs, as illustrated in Fig. III.5b ${ }^{3}$. For exponential $\mathrm{SBP}_{\mathrm{LSB}}$, the stellar luminosity density $l_{\mathrm{E}}\left(R^{\star}\right)$ is related to $\mu_{E, 0}$ and $\alpha_{E}$ by Eq. (III.24) (see the dotted lines in Fig. III.5b). For a given $l_{\mathrm{E}}\left(R^{\star}=2 \alpha_{\mathrm{E}}\right)$, Eqs. (III.24) and (III.4) allow therefore the calculation of $\mu_{E, 0}\left(M_{\mathrm{E}}\right)$ and $\alpha_{E}\left(M_{\mathrm{E}}\right)$ through Eqs. (III.27) and (III.28),

$$
\begin{aligned}
\mu_{\mathrm{E}, 0}\left(M_{\mathrm{E}}, l_{\mathrm{E}, 2 \alpha}\right)= & \frac{1}{3} M_{\mathrm{E}}-\frac{5}{3} \log l_{\mathrm{E}, 2 \alpha}+21.632 \\
& +\frac{2}{3} M_{\odot}^{\text {abs }}+\frac{5}{3} \log C_{2 \alpha} \\
\log \alpha_{\mathrm{E}}\left(M_{\mathrm{E}}, l_{\mathrm{E}, 2 \alpha}\right)= & -\frac{2}{15} M_{\mathrm{E}}-\frac{1}{3} \log l_{\mathrm{E}, 2 \alpha}-0.388 \\
& +\frac{2}{15} M_{\odot}^{\text {abs }}+\frac{1}{3} \log C_{2 \alpha}
\end{aligned}
$$

with $l_{\mathrm{E}, 2 \alpha}$ in units of $L_{\odot, J} \mathrm{pc}^{-3}, \alpha_{E}$ in pc, $\mu_{E, 0}$ in mag/ $\square^{\prime \prime}$, and $C_{2 \alpha}=C_{1}\left(R^{\star}=2 \alpha_{\mathrm{E}}\right)$.

\footnotetext{
${ }^{2}$ Uncertainties of $P_{23}$ are chosen relatively large, since this value depends critically on the exact determination of the $\mathrm{SBP}_{\mathrm{LSB}}$ shape, e.g. the detection of a type V SBP.

${ }^{3}$ For consistency, and keeping in mind that type V SBP ${ }_{\mathrm{LSB}}$ may have not have been detected whenever their cutoff-radius $b \alpha_{E}$ is smaller than the starburst plateau radius $P_{23}$, we calculate $M_{\mathrm{E}}$ throughout this section from $\mu_{E, 0}$ and $\alpha_{E}$ according to Eq. (III.4) for all sample BCDs.
} 

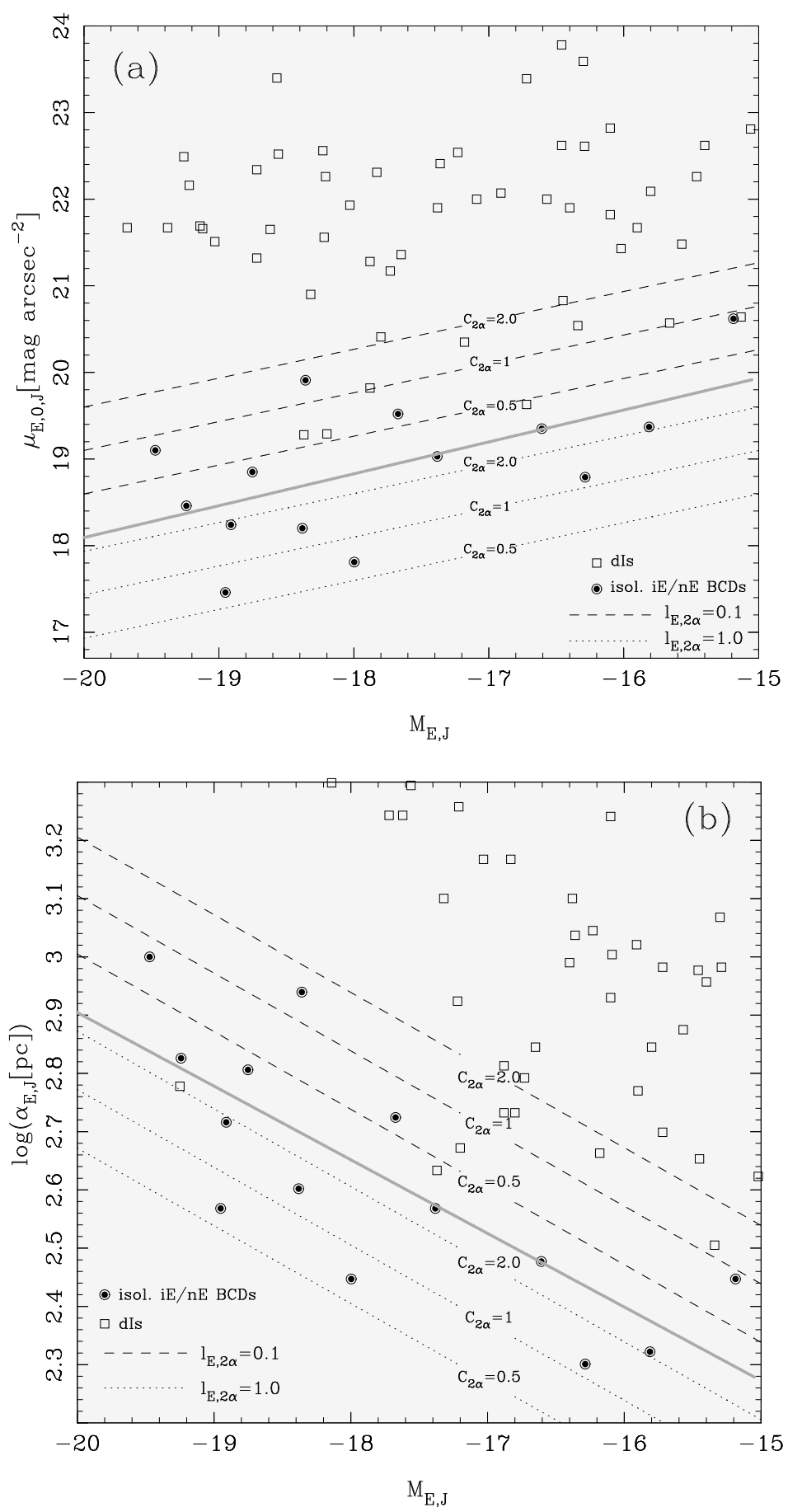

Fig. III.6. a) Extrapolated $J$ central surface brightness $\mu_{E, 0}$ and b) $J$ exponential scale length $\alpha_{E}$ of the LSB components of the isolated BCDs studied in Sect. III.5.2, plotted against the absolute magnitude $\mathrm{M}_{\mathrm{E}, J}$ of their LSB components as calculated from Eq. (III.4) (filled circles). Heavy gray lines show linear fits to the BCD data. Overplotted are lines of constant stellar luminosity density at $R^{\star}=2 \alpha_{E}, l_{\mathrm{E}, 2 \alpha}=0.1 L_{\odot, J} \mathrm{pc}^{-3}$ (dashed lines) and $1.0 L_{\odot, J} \mathrm{pc}^{-3}$ (dotted lines) as obtained from Eqs. (III.27) and (III.28). The effect of deprojection deviation factors $C_{2 \alpha}=0.5$ and 2 is shown. Squares show, for comparison, dIs from Binggeli \& Cameron (1991) and Patterson \& Thuan (1996). 
If collective, burst-like star formation requires a minimum $l_{\text {crit }}$, then only those dwarf galaxies should undergo a BCD phase, which at least at the centers of their LSB hosts show a sufficiently high stellar luminosity density.

We do not consider $l_{\mathrm{E}}$ at $R^{\star}=0^{\prime \prime}$ here, since this value may vary strongly for slightly differing $(b, q)$ and geometries (see Eq. III.26), and has a very local character. Instead, Eqs. (III.27) and (III.28) are evaluated for different $l_{\mathrm{E}, 2 \alpha}=l_{\mathrm{E}}\left(R^{\star}=2 \alpha_{\mathrm{E}}\right)$, a radius where the expected deviations $C_{2 \alpha}=C_{1}\left(R^{\star}=2 \alpha_{\mathrm{E}}\right)$ of Eq. (III.24) differ much less strongly from unity than $C_{1}\left(R^{\star}=0\right)$. The transition from $l_{\mathrm{E}}\left(R^{\star}=0\right)$ to $l_{\mathrm{E}, 2 \alpha}$ is justified, since both values are proportional to each other for a given pair of $b, q$. This choice furthermore accounts for the fact that starburst activity in BCDs is not confined to a central spot, but occurs over an extended volume of the LSB host.

The lines of constant $l_{\mathrm{E}, 2 \alpha}$, shown in Fig. III.6, run roughly parallel to the BCD sequence. Equations (III.27) and (III.28) yield slopes $\mu_{E, 0} \propto 0.33 M_{\mathrm{E}}$ and $\log \alpha_{E} \propto$ $-0.13 M_{\mathrm{E}}$, which compare well to those from linear fits to the data, $\mu_{E, 0} \propto(0.37 \pm$ $0.15) M_{\mathrm{E}}$ and $\log \alpha_{E} \propto(-0.13 \pm 0.03) M_{\mathrm{E}}$. The approximate lower limit to the data, $l_{\mathrm{E}, 2 \alpha} \sim 0.1 L_{\odot, J} \mathrm{pc}^{-3}$, estimated allowing for moderate deprojection deviation factors $C_{2 \alpha}$, is slightly lower than the estimated $l_{\text {crit }}$ of $\sim 0.2 L_{\odot, J} \mathrm{pc}^{-3}$. This difference reflects that the observed limiting radii of SF, $P_{23}$, are mostly $<2 \alpha_{E}$ (cf. Fig. III.7), therefore $l_{\mathrm{E}}\left(P_{23}\right)$ are slightly larger than $l_{\mathrm{E}, 2 \alpha}$ (Eq. III.24).

Apparently, the observed segregation of starbursting from quiescent gas-rich dwarfs in the $\mu_{E, 0}-M_{\mathrm{E}}$ and $\alpha_{E}-M_{\mathrm{E}}$ parameter space reflects different central stellar luminosity densities of the LSB host, with BCDs being associated with lines of of large density $l_{\mathrm{E}, 2 \alpha}$. These results support the hypothesis proposed in Sect. III.5.2.1, that a necessary condition for the ignition of starburst activity in gas-rich dwarf galaxies is a minimum stellar density in their LSB host galaxy. The existence of some dIs with $l_{\mathrm{E}, 2 \alpha}$ higher than the putative lower limit of starburst activity is consistent with the assumption that such a lower limit does only allow, but not force starburst activity (see the previous subsection).

\section{III.5.2.3 Clues to the relations between BCDs and dIs}

To summarize, the evidence of Figs. III.6a and b supports the hypothesis of a minimum stellar mass density, needed to ignite burst-like star-forming activity within the gaseous halo of an isolated dwarf galaxy. Such a scenario, if proven, even offers an explanation for the observed slopes of the $\alpha_{E}$ vs $M_{\mathrm{E}}$ and $\mu_{E, 0}$ vs $M_{\mathrm{E}}$ relations of BCDs, as well as for the systematic separation of these systems from dIs with respect to the structural properties of their LSB host galaxy. As was already discussed by Papaderos (1998), dIs are those SF dwarf galaxies which show diffuse stellar mass distributions, i.e. lower densities within their central regions, not allowing (or not forcing) a starburst event. BCDs, on the contrary, occupy those regions in the parameter space which allow for sufficiently high stellar mass densities to give rise to burst-like SF activity.

We can, however, not explain the relatively narrow range of $l_{\mathrm{E}, 2 \alpha}$ of the BCDs' LSB hosts: although the spacing of the lines of constant $l_{\mathrm{E}, 2 \alpha}$ is logarithmic in Fig. III.6, there seems to be a limit of $l_{\mathrm{E}, 2 \alpha} \lesssim 2 L_{\odot, J} \mathrm{pc}^{-3}$. If BCDs represent the most compact 
objects among star-forming dwarf galaxies, then it is puzzling why the parameter space of their central stellar density is so narrow, compared to the entire range of structural properties covered by dwarf galaxies (see Fig. 13 in Papaderos et al. 2002), and roughly delimited by a line of constant stellar LSB density.

One possible explanation is that the luminosity density of the starburst component may increase with $l_{\mathrm{E}, 2 \alpha}$ (cf. Sect. III.5.2.5). This relation may imply that the mechanical energy ejection rate from the starburst increases with the compactness of the host galaxies. Such a stellar density-dependent starburst activity could therefore act as a regulating agent, forcing compact mass distributions to adiabatically expand (P96b), or even entirely disrupting extremely low-mass objects.

The absence of dI-classified objects in regions of high central density $l_{\mathrm{E}, 2 \alpha}$ (Fig. III.6) may be important to scenarios of evolutionary interrelations between BCDs and dIs. P96b concluded from the structural differences between the stellar hosts of either object class that an evolutionary link between dIs and BCDs would require an adiabatic and homologous redistribution of the stellar mass within the LSB component, in response to a global change in the gravitational potential of these systems within their Holmberg radii. Such a change in the gravitational well can result from a large-scale $\mathrm{H}$ I inflow preceding the burst and, subsequently, the starburst-driven ejection of gas into the halo. The a posteriori discovery of a much higher central $\mathrm{H} \mathrm{I}$ concentration in BCDs than in dIs by van Zee et al. (1998,2001) and Simpson \& Gottesmann (2000) strongly support the latter hypothesis. This assumption is also compatible with the limiting central density of the stellar host which may be due to a starburst-induced regulation (see the previous paragraph), and with the possible relation between the flattening

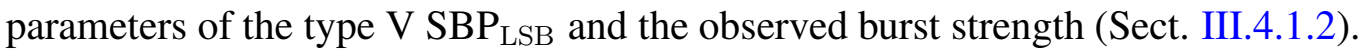

P96b described two limiting cases, depending on whether or not Dark Matter dominates on a radial scale comparable to the Holmberg radius of a BCD: a) a static LSB distribution is realized when Dark Matter entirely dominates within the optical size of a BCD, "freezing" its global gravitational potential. In that case, no evolutionary connection between BCDs and dIs is possible. b) the aforementioned dynamic evolutionary $\mathrm{BCD} \leftrightarrow \mathrm{dI}$ transition, which is only possible if DM does not dominate within the Holmberg radius.

This first scenario was considered most likely by e.g. Meurer et al. (1998) on the assumption that DM dominates the dynamics of both BCDs and dIs at all radii. However, the static scenario favoured by Meurer et al. would imply that also the most compact BCDs $\left(l_{\mathrm{E}, 2 \alpha} \gtrsim 1 L_{\odot, J} \mathrm{pc}^{-3}\right)$ retain their compact structure in between starburst episodes. If these interburst phases are sufficiently long to allow the bursts to fade significantly (a few $10^{8} \mathrm{yr}$ ), then one would expect a sizeable number of quiescent gas-rich dwarf galaxies, most likely classified as compact dIs or blue dEs, with structural properties indistinguishable from those of BCDs.

Such a rich population of compact post-starburst dwarf galaxies remains elusive; neither such compact dIs, nor a significant number of comparably compact blue dEs (see Fig. 13 of Papaderos et al. 2002) have been discovered as yet. This observational result contradicts the static scenario of total DM dominance, unless the most compact BCDs are almost always in a state of sufficiently high SF activity to be classified as 
BCDs. This would require that BCDs have maintained strong SF activity over their entire lifetime of several Gyr, with at most short phases of lowered activity. The dynamic hypothesis received support by recent dedicated kinematic studies of BCDs (Walter et al. 1997, Gil de Paz et al. 1999, 2002) which argue against a universal dominance of DM within the optical extent of these systems.

\section{III.5.2.4 The dependence of the starburst extent on the luminosity of the LSB host}

From a deprojection of the exponential intensity profiles of the LSB hosts of BCDs, P96b found the central stellar mass density in these systems to increase towards fainter $M_{\mathrm{E}}$. At the same time, the relative extent of the starburst within the LSB host was found to increase. Papaderos (1998) hypothesized that the latter relation could be a consequence of the former.

On the hypothesis of the existence of an $l_{\text {crit }}$ above which burst-like SF can occur, an indicative upper limit to the spatial extent of the starburst can be calculated for a given set of structural properties for the LSB host. We will in the following explore whether this assumption can reproduce the observed relations between the extent of the starburst and the LSB host luminosity.

For given $l_{\mathrm{E}, 2 \alpha}$ and $l_{\text {crit }}$, the radius $R_{\text {crit }}^{\star}$ where $l_{\mathrm{E}}\left(R_{\text {crit }}^{\star}\right)=l_{\text {crit }}$, i.e. within which $\mathrm{SF}$ is expected to occur, can be calculated from Eqs. (III.24), (III.27) and (III.28):

$$
\begin{aligned}
& \frac{R_{\text {crit }}^{\star}}{\alpha_{\mathrm{E}}}\left(l_{\mathrm{E}, 0}, l_{\text {crit }}\right)= \\
& 0.9 \ln 10\left[\log \left(\frac{l_{\mathrm{E}, 2 \alpha}}{l_{\text {crit }}}\right)+0.965+\log \left(\frac{C_{1}}{C_{2 \alpha}}\right)\right]
\end{aligned}
$$

so that $\frac{R_{\text {crit }}^{\star}}{\alpha_{\mathrm{E}}}\left(l_{\mathrm{E}, 0}, l_{\text {crit }}\right)$ should be independent of $M_{\mathrm{E}}$.

In Fig. III.7a we compare the plateau radius, normalized to the scale length of the underlying LSB component $\left(P_{23} / \alpha_{E}\right)$ vs. $M_{\mathrm{E}}$. Indeed, there is no obvious dependence on $M_{\mathrm{E}}$, as predicted by Eq. (III.29). Also the range of values of $\left(P_{23} / \alpha_{E}\right)$ can be approximately described by Eq. (III.29), if the estimated $l_{\text {crit }}$ of $0.2 L_{\odot, J} \mathrm{pc}^{-3}$ is adopted. Values of $\left(P_{23} / \alpha_{E}\right)$, calculated for lower and upper limits of $l_{\mathrm{E}, 2 \alpha}, 0.1$ and $1 L_{\odot, J} \mathrm{pc}^{-3}$ (see above), reproduce the lower and upper limits of the data, for moderate deprojection deviation factors $C_{1}$ and $C_{2 \alpha}$ (long-dashed and dotted lines in Fig. III.7a). The deviant point represents Haro 14, a BCD with large $(b, q)=(3.6,0.94)$, for which the observed $\left(P_{23} / \alpha_{E}\right)$ is probably overestimated.

On the assumption of an $l_{\text {crit }}$, also the the star formation concentration index $C I$, introduced by P96b, can be calculated. This value describes the fractional area of the LSB host covered by the starburst, with both areas measured at an isophotal level $\mu_{\text {iso }}$ (25 $\mathrm{B} \mathrm{mag} / \square^{\prime \prime}$ in P96b and $23 \mathrm{~J} \mathrm{mag} / \square^{\prime \prime}$ in N03a,b).

$$
C I=1-\left(\frac{P_{\mu_{\text {iso }}}}{E_{\mu_{\text {iso }}}}\right)^{2}
$$



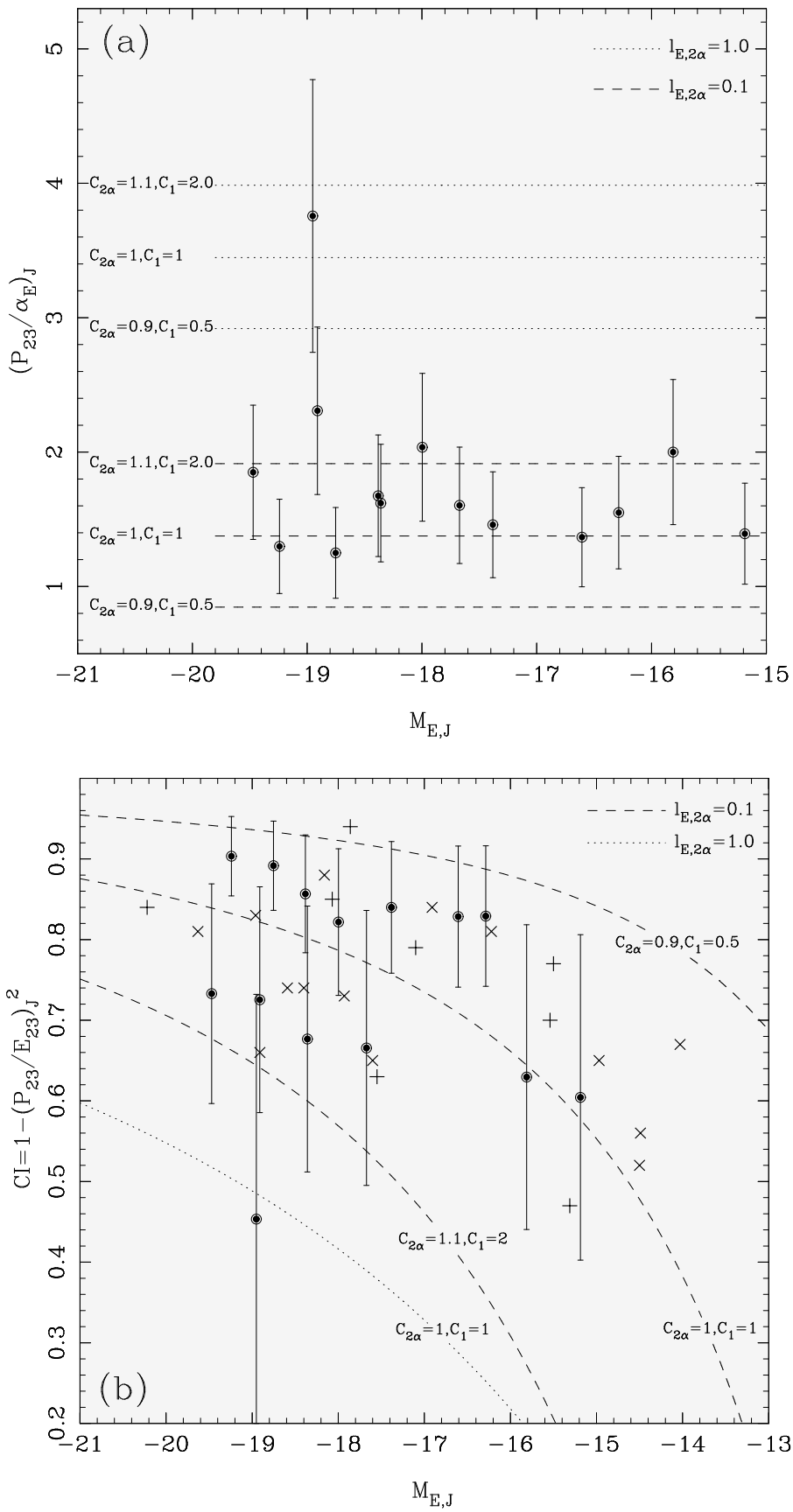

Fig. III.7. a) Radius $P_{23}$ of the starburst component, normalized to the exponential scale length of the underlying LSB component $\left(\alpha_{E}\right)$, vs. absolute $J$ magnitude of the LSB host $\left(M_{\mathrm{E}}\right)$ for all isolated BCDs considered in Sect. III.5.2. Horizontal lines show the $\left(P_{23} / \alpha_{E}\right)$ vs. $M_{\mathrm{E}}$ relations predicted by Eq. (III.29), for $l_{\text {crit }}=0.2 L_{\odot, J} \mathrm{pc}^{-3}$ and different $l_{\mathrm{E}, 2 \alpha}, C_{2 \alpha}$ and $C_{1}$ as indicated at each line. b) $J$ band star formation concentration index $C I$ vs. $M_{\mathrm{E}}$ of the isolated BCDs considered in Sect. III.5.2 (filled circles). For comparison, we show BCDs investigated in the $B$ band by P96a ('X') and Noeske 1999 ('+'). For those objects, the $M_{\mathrm{E}}$ derived in the $B$ band were transformed to the $J$ band adopting a $(B-J)_{\mathrm{LSB}}$ of 2 . Thin lines show the $C I$ predicted by Eq. (III.31), for $l_{\text {crit }}=0.2 L_{\odot, J} \mathrm{pc}^{-3}$ and $l_{\mathrm{E}, 2 \alpha}, C_{2 \alpha}$ and $C_{1}$ as indicated. Errors of the observed $C I$ are calculated taking into account uncertainties in $\mu_{E, 0}, \alpha_{E}$ and $P_{23}$ (Sect. III.5.2.1), as well as typical uncertainties of $5 \%$ in the determination of the isophotal radius $E_{23}$ of the underlying LSB host. 
Here, $P_{\mu_{i s o}}$ and $E_{\mu_{i s o}}$ respectively denote the isophotal radii of the starburst (plateau) and the LSB host. Using Eq. (III.2), this can be rewritten as

$$
\begin{aligned}
C I & \left(M_{\mathrm{E}}, l_{\mathrm{E}, 2 \alpha}, l_{\text {crit }}\right) \\
& =1-\left[\frac{1.086\left(\frac{R_{\text {crit }}^{\star}}{\alpha_{\mathrm{E}}}\right)\left(M_{\mathrm{E}}, l_{\text {crit }}, l_{\mathrm{E}, 2 \alpha}\right)}{\mu_{\text {iso }}-\mu_{\mathrm{E}}\left(M_{\mathrm{E}}, l_{\mathrm{E}, 2 \alpha}\right)}\right]^{2}
\end{aligned}
$$

This equation can be evaluated using the results from Eqs. (III.29) and (III.27), and therefore also allows for the consideration of different $l_{\mathrm{E}, 2 \alpha}$ and deviation factors $C_{2 \alpha}$ and $C_{1}$, which enter into the latter both equations. The progression of Eq. (III.31) is shown in Fig. III.7b, evaluated for the same $l_{\text {crit }}$ and upper and lower limits of $l_{\mathrm{E}, 2 \alpha}$ as Eq. (III.29). Observed data, derived from the BCD sample considered in the present section, are shown by filled circles. Similarly to Fig. III.7a, the upper and lower limits of $l_{\mathrm{E}, 2 \alpha}$, describing the range of the structural parameters of BCD hosts, can reproduce the observed range of $C I$ at a given $M_{\mathrm{E}}$. Again, the outlying point represents Haro 14 (see above).

The "+" and " $x$ " signs in Fig. III.7b mark $B$ band data from P96a and Noeske (1999), with $M_{\mathrm{E}}$ approximately transformed to the $J$ band adopting a mean $B-J=2$ (see Table 3 in both N03a and N03b). Also the distribution of those data can be well reproduced by Eq. (III.31), adopting the range of parameters we have estimated above.

We note here that P96b and Noeske (1999) assumed a linear relation to quantify the average dependence of the $C I$ on $M_{\mathrm{E}}$. On the assumption of $l_{\text {crit }}$ and exponential LSB host galaxies, Eq. (III.31) shows that this relation is in fact non-linear. However, the deviations from linearity are not well discernible in observational data within the $M_{\mathrm{E}}$ interval covered by BCDs, due to the intrinsic scatter of the $C I-M_{\mathrm{E}}$ relation, which originates from the intrinsic dispersion in the LSB hosts' structural properties at a given $M_{\mathrm{E}}$ (Sect. III.5.2.2).

\section{III.5.2.5 Luminosity densities of the starburst and the stellar host galaxy}

As was shown by P96b, the relative extent of the starburst within the host galaxy $\left(P_{23} / E_{23}\right)$, and hence its fractional volume $\left(P_{23} / E_{23}\right)^{3}$ within the LSB host, is on average dependent on $M_{\mathrm{E}}$ (cf. Fig. III.7 \& Eq. III.30 in the present paper). The burst-to-host galaxy light ratios show, however, no correlation with $M_{\mathrm{E}}\left(L_{\mathrm{SB}} / L_{\mathrm{E}} \propto\right.$ $(-0.02 \pm 0.13) M_{\mathrm{E}}$, see Fig. III.8a). This indicates that the luminosity of the starburst, $L_{S B}$, does not depend on its volume $\left(\propto P_{23}^{3}\right)$ alone. Instead, also the luminosity density $\overline{l_{S B}}$ of the starburst, averaged over its volume,

$$
\overline{l_{S B}}=\frac{3 L_{S B}}{4 \pi\left(P_{23}\right)^{3}}
$$

should systematically depend on the properties of the stellar host. If we adopt that, on average, $L_{S B} \propto L_{\mathrm{E}}$, as indicated by Fig. III.8a, and use $L_{\mathrm{E}} \propto I_{\mathrm{E}, 0}$ (Eq. III.4) and $I_{\mathrm{E}, 0} \propto l_{\mathrm{E}, 0} \alpha_{E}$ (Eq. III.24), then Eq. (III.32) leads to a proportionality of the form 

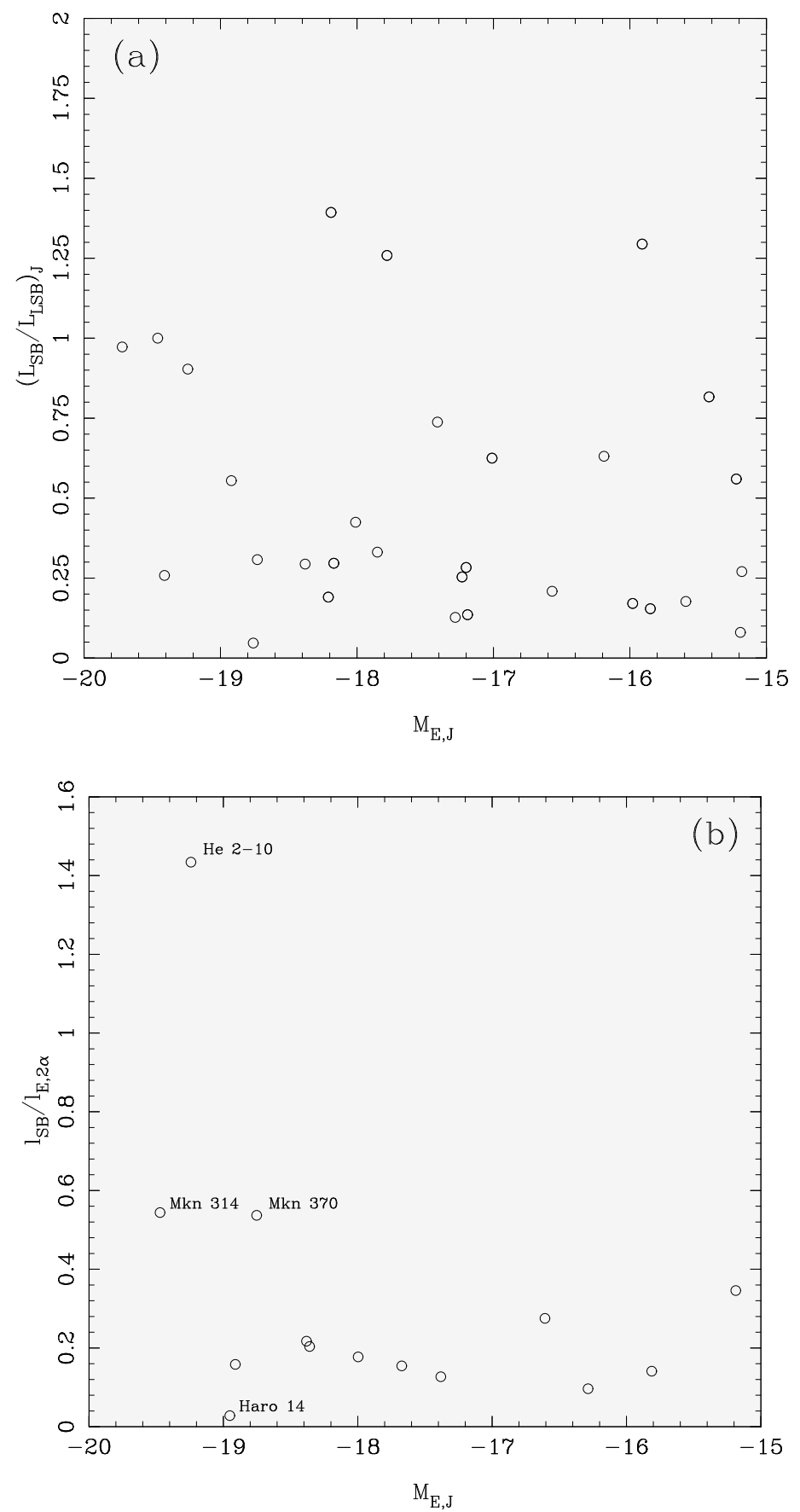

Fig. III.8. a) Luminosity ratio $L_{\mathrm{SB}} / L_{\mathrm{E}}$ of the starburst and LSB component in the $J$ band vs. absolute magnitude of the stellar LSB host galaxy. b) Ratio of the mean luminosity density $l_{\mathrm{SB}}$ of the starburst component inside its radius $P_{23}$ and the luminosity density $l_{\mathrm{E}, 2 \alpha}$ of the underlying LSB host galaxy at a galactocentric radius $R=2 \alpha_{E}$ (see Sect. III.5.2.5) vs. the absolute $J$ magnitude $M_{\mathrm{E}, \mathrm{J}}$ of the LSB host. 


$$
\overline{l_{S B}} \propto \frac{l_{\mathrm{E}, 0}}{\left(\frac{P_{23}}{\alpha_{\mathrm{E}}}\right)^{3}}
$$

with $l_{\mathrm{E}, 0}$ denoting the central luminosity density of the stellar host.

As was shown in Sect. III.5.2.4, $\left(P_{23} / \alpha_{\mathrm{E}}\right)$ is approximately constant, varying around its mean value by a factor of $\lesssim 1.5$. The ratio $\left(\overline{l_{S B}} / l_{\mathrm{E}, 0}\right)$ should therefore also be constant, on average, with intrinsic variations of the order of the variations of $\left(P_{23} / \alpha_{\mathrm{E}}\right)$ to the 3rd power. Additional scatter could be introduced by e.g. the strong variability of $L_{S B}$ with the burst age.

To test this hypothesis, we restrict ourselves to the subsample investigated in this Sect. III.5.2, i.e. for which the central luminosity density can be estimated using Eq. (III.24). As justified in Sect. III.5.2.2, we use instead of the central luminosity density the value $l_{\mathrm{E}}$ at the radius $R^{*}=2 \alpha_{\mathrm{E}}$, which typically gives an better estimation of the stellar density in the central region of the stellar host.

The resulting ratios $\left(\overline{l_{S B}} / l_{\mathrm{E}, 2 \alpha}\right)$ indeed seem to form a roughly constant sequence (Fig. III.8b). The outlying points correspond to Haro 14, for which $l_{\mathrm{E}, 2 \alpha}$ may be overestimated, Mkn 314, a possible polar ring galaxy (Whitmore et al. 1990), Mkn 370, which shows a morphology similar to Mkn 314 (C01a,b) and for which an exponential SBP $\mathrm{LSB}_{\mathrm{LB}}$ may not be an adequate model (Tab. III.1), and He 2-10, displaying an extremely strong burst. If these points are excluded, one obtains a mean value $\left\langle\overline{l_{S B}} / l_{\mathrm{E}, 2 \alpha}\right\rangle=0.19$, with a sample standard deviation of 0.09 . Individual $\left(\overline{l_{S B}} / l_{\mathrm{E}, 0}\right)$ show no significant dependency on $M_{\mathrm{E}}$.

This result may suggest a close relation between the central density of the stellar host galaxy and the amplitude of SF activity for a large fraction of BCDs. The average dependency $\left(\overline{l_{S B}} \propto l_{\mathrm{E}, 0}\right)$ would be surprisingly simple, and receives support from the results by Papaderos (1998). This author found the burst strength as derived from ROSAT X-ray observations, $L_{X} / L_{B}$, to scale with the central stellar mass density of the host galaxy.

\section{III.5.2.6 A modified Jeans-criterion as a possible explanation?}

An appealing scenario, relating the occurrence and amplitude of starbursts to the mass density of a compact stellar background, was proposed by Loose \& Fricke (1980) and Loose et al. (1982), and discussed in the framework of the X-ray properties of starbursts in BCDs by Papaderos (1998). The former authors showed that the tidal field of a stellar background with an average mass density $\rho_{*}$ can modify the Jeans-criterion, such that individual gas clouds are stabilized against collapse and subsequent SF until their density $\rho_{c}$ exceeds $\rho_{*}$.

This mechanism could account in a simple way for a minimum density $l_{\text {crit }}$ of the LSB component to host starburst activity. Such a limit would correspond to a $\rho_{*}$ in the presence of which the $\rho_{c}$ of individual clouds can grow sufficiently large to give rise to a collective star-forming episode with the amplitude of a starburst, a spatial extent of several hundred pc, formation of SSCs, and a resulting thermalization of the interstellar medium on a kpc scale. 
The observed proportionality between $\overline{l_{\mathrm{SB}}}$ and $l_{\mathrm{E}, 0}$ or $l_{\mathrm{E}, 2 \alpha}$ (Eq. III.33) could be understood in a similar way. A proportionality between $\rho_{c}$ and $\rho_{*}$ would, averaged over a sufficiently large volume and number of clouds, imply the observed proportionality between $\overline{l_{\mathrm{SB}}}$ and $\rho_{*}$.

\section{III.5.2.7 Summarizing remarks}

In this section, we have tested the hypothesis of a critical stellar threshold density, above which burst-like SF activity is possible in gas-rich dwarf galaxies. We have restricted ourselves to probably isolated BCDs with a relatively regular morphology. On the assumption that their stellar host galaxies have exponential intensity distributions, and can be on average approximated by spheroidal geometry, this $l_{\text {crit }}$-hypothesis can consistently account for several relations empirically found for BCDs.

We wish to emphasize that the arguments supporting the hypothesis of an $l_{\text {crit }}$ do not rely on a certain value of this threshold. Both the predictions of Eqs. (III.27) and (III.28), and those of Eqs. (III.29) and (III.31) support the $l_{\text {crit }}$ - hypothesis by reproducing the slopes and progressions of the observed relations, irrespective of the assumed value of $l_{\text {crit }}$. Varying $l_{\text {crit }}$ would merely introduce effects which are equivalent to those of deprojection deviation functions $C_{1}$ and $C_{2 \alpha}$ which differ from one (Fig. III.7), and to slight variations of the parameter $l_{\mathrm{E}, 2 \alpha}$, both illustrated in the figures III.6 and III.7.

The results presented throughout this Sect. III.5.2 strongly suggest that the stellar mass density of the LSB host either forms itself, or closely traces, a prime parameter governing the starburst activity in gas-rich dwarf galaxies. In view of the data uncertainties and scatter, as well as the assumptions and approximations made in the present investigation, the importance of further parameters should however not be disregarded. In this respect, it will be particularly important to consider the distribution of the neutral gas which, as shown in van Zee et al. $(1998,2001)$, is more dense in BCDs than in dIs.

We further note that, as shown in Fig. III.3, the cutoff radius $b \alpha_{\mathrm{E}}$ within which a type V LSB hosts flattens off is generally close to $P_{23}$. Both radii correspond therefore to similar $l_{\mathrm{E}}$, and to an approximate spatial limit $R_{\text {crit }}^{\star}$ of the SF activity. An interplay between the central flattening of type V LSB hosts and star-forming activity appears therefore also likely (cf. Sect. III.4.1.2), similar to what has been observed for spiral galaxies (Pohlen et al. 2002b) and in a dI (Hunter 2002) (cf. Sect. III.4). This hypothesis may also receive support from the observed trend between the flattening parameters

$b, q$ of the type V LSB hosts and the starburst strength, reported in Sect. III.4 of this paper.

\section{III.6 Summary and Conclusions}

We have analyzed the results of NIR surface photometry of a sample of 33 Blue Compact Dwarf Galaxies (BCDs) and more luminous Blue Compact Galaxies, compiled from previous parts of the present series of papers. Compared to previous studies in optical wavelengths, these NIR data allow to better separate the luminosity contribution 
of the underlying old stellar LSB host galaxy from that of the young stellar population, formed in the ongoing and recent star-forming activity. The luminosity fraction of both latter major stellar populations has been determined and discussed with regard to previous determinations in the visible light. The available NIR data set has been further employed to systematically study for the first time "type V" surface brightness profiles of the stellar host galaxy $\left(\mathrm{SBP}_{\mathrm{LSB}}\right)$, with respect to their frequency, parametrization and possible relation to other observables of BCDs. Specifically, we have quantitatively investigated whether the density profile of the old stellar host galaxy is influencing the occurrence, spatial extent and volume density of star-forming (SF) activity. The results of this study were discussed in view of possible evolutionary connections between BCDs and dwarf irregulars. Our main conclusions may be summarized as follows:

1. The structural parameters of the $\mathrm{SBP}_{\mathrm{LSB}}$ of the stellar host galaxies, derived from exponential fits to their outer regions, are generally in agreement in optical and NIR bands. Our data confirm the basic trends between the central surface brightness $\mu_{E, 0}$, exponential scale length $\alpha_{E}$ and absolute magnitude $M_{\mathrm{E}}$ of the LSB host galaxy, discovered previously from optical $B$ data. They also show that, at equal $M_{\mathrm{E}}$, the underlying stellar LSB host of BCDs shows a significantly more compact density distribution than in dwarf irregulars.

2. We infer a fraction of $55 \pm 16 \%$ of BCDs with a type $\mathrm{V}$ profile in their underlying LSB host galaxy. The LSB component of these systems shows an exponential slope in its outer part, and a conspicuous intensity flattening inside a cutoff radius which is typically equivalent to 1-3 exponential scale lengths. Such type V profiles have been detected in 9 out of 10 irregular (iI/iI,C), but only in 4 out of 13 elliptical (iE/nE) BCDs. However, we discuss a number of arguments supporting the view that this apparent bias arises mainly from observational selection effects and that the actual fraction of type $\mathrm{V}$ profiles in $\mathrm{iE} / \mathrm{nE}$ systems is considerably larger. Extended starburst emission in the latter systems outshines the inner part of the LSB component, preventing a reliable determination of the intensity profile of the underlying old stellar population. Indeed, the strong observational bias against the detection of type $\mathrm{V}$ profiles is reflected on the fact that whenever such a distribution is detected, it generally shows a characteristic cutoff radius comparable to, or larger than, the radius of the starburst component.

3. Type $\mathrm{V}$ profiles have been approximated using a modified exponential distribution (hereafter denoted med) proposed in Papaderos et al. (1996a). The intensity distribution given by this formula depends, in addition to the extrapolated central surface brightness $\mu_{E, 0}$ and the exponential scale length $\alpha_{E}$, on the cutoff-radius $b \alpha_{E}$ where a type $\mathrm{V}$ profile levels off, and on its relative intensity depression $q$, compared to $\mu_{E, 0}$. For most type $\mathrm{V} \mathrm{SBP}_{\mathrm{LSB}}, q$ has been found to be non-linearly related to the cutoffradius $b$. Neither $b$ nor $q$ show a systematic dependence on $M_{\mathrm{E}}, \alpha_{E}$ or $\mu_{E, 0}$. However, there is a hint for either parameter to increase for BCDs with higher starburst-to-LSB luminosity ratios $\left(L_{\mathrm{SB}} / L_{\mathrm{E}}\right)$.

4. The starburst-to-LSB luminosity ratio $L_{\mathrm{SB}} / L_{\mathrm{E}}$ shows a large scatter, with mean values of $\sim 0.6$ and $\sim 1.8$ in the $J$ and $B$ bands, respectively, and reveals no significant correlation with $M_{\mathrm{E}}$. We argue that profile decomposition in BCDs with a type $\mathrm{V}$ 
$\mathrm{SBP}_{\mathrm{LSB}}$, using a purely exponential LSB model, may on average underestimate the true $L_{\mathrm{SB}} / L_{\mathrm{E}}$ ratio by a factor of $\sim 0.5$ in the $B$ band.

5. For a subsample of typical, isolated BCDs, we estimate the stellar luminosity density distributions of their old stellar host galaxies. Based on these results, we raise the hypothesis of a critical stellar luminosity density of the host galaxy, $l_{\text {crit }}$, above which burst-like SF activity becomes possible. We empirically infer the $l_{\text {crit }}$ to $\sim 0.2 \pm$ $0.1 L_{\odot, J} \mathrm{pc}^{-3}$, corresponding to a mass density of $\sim 0.4 \pm 0.2 M_{\odot} \mathrm{pc}^{-3}$ for an old stellar population with subsolar metallicity.

For stellar host galaxies with an exponential $\mathrm{SBP}_{\mathrm{LSB}}$ and a roughly spheroidal geometry, this hypothesis of an $l_{\text {crit }}$ can explain the observed separation of BCDs and dIs in the $\mu_{E, 0}-M_{\mathrm{E}}$ and $\mu_{E, 0}-\alpha_{E}$ parameter space. The same assumption allows to analytically predict the slopes, and to partially understand the intrinsic spread, of the $\mu_{E, 0}$ $-M_{\mathrm{E}}$ and $\alpha_{E}-M_{\mathrm{E}}$ relations observed for BCDs. In the same vein, we can reproduce the observed dependence of the star formation concentration index on $M_{\mathrm{E}}$, as well as the independence of the linear starburst extent of $M_{\mathrm{E}}$.

6. For a major fraction of BCDs, the data suggest that the mean luminosity density of the starburst scales with the central density of the LSB host galaxy. We propose that the observed relations between the starburst activity and the stellar mass density of the host galaxy might be explained by a modification of the Jeans-criterion in the presence of a compact stellar background, similar to a model put forward by Loose \& Fricke (1980).

7. In the $\mu_{E, 0}-\alpha_{E}$ parameter space of stellar hosts of gas-rich star-forming dwarf galaxies, the most compact configurations are almost invariably identifiable with BCDs, i.e. objects undergoing vigorous, collective star-forming activity. The almost complete lack of comparably compact objects in a quiescent state argues against a static scenario of dwarf galaxy evolution, commonly ascribed to an extraordinarily dense Dark Matter halo that dominates the mass at all radii. The current results, in conjunction with different lines of evidence in the optical and radio wavelengths, support the hypothesis of an adiabatic redistribution of stellar mass in the LSB component of BCDs, in response to a large scale inflow and subsequent starburst-driven expansion of the massive $\mathrm{H}$ I halo of these systems.

Acknowledgement. Research by K.G.N. has been supported by the Deutsche Forschungsgemeinschaft (DFG) grants FR325/50-1 and FR325/50-2. Research by P.P. and K.J.F. has been supported by the Deutsches Zentrum für Luft- und Raumfahrt e.V. (DLR) under grant 50 OR 9907 7. L.M.C. acknowledges support from the European Community Marie Curie grant HPMF-CT-2000-00774. We thank Dr. Uta Fritze - v. Alvensleben, P. Anders, J. Bicker and J. Schulz for kindly providing the GALEV models. This research has made use of the NASA/IPAC Extragalactic Database (NED) which is operated by the Jet Propulsion Laboratory, CALTECH, under contract with the National Aeronautic and Space Administration. This publication makes use of data products from the Two Micron All Sky Survey, which is a joint project of the University of Massachusetts and the Infrared Processing and Analysis Center/California Institute of Technology, funded by the National Aeronautics and Space Administration and the National Science Foundation. 


\section{References}

Balkowski, C., Chamaraux, P. \& Weliachew, L. 1978, A\&A, 69, 263

Barazza, F. D., Binggeli, B. \& Prugniel, P. 2001, A\&A, 373, 12

Binggeli, B., Cameron, L. M. 1991, A\&A, 252, 27

Cairós, L. M. 2000, PhD Thesis, Univ. de La Laguna

Cairós, L. M., Vílchez, J. M., González Pérez, J., Iglesias-Páramo, J., \& Caon, N. 2001, ApJS, 133, 321

Cairós, L. M., Caon, N., Papaderos, P., Noeske, K. G., V’ ilchez, J. M., García Lorenzo, B., Muñoz-Tuñon, C. 2002a, submitted (C03)

Drinkwater, M. \& Hardy, E. 1991, AJ, 101, 94

5950

Fricke, K. J., Izotov, Y. I., Papaderos, P., Guseva, N. G., Thuan, T. X. 2001, AJ, 121, 169 (F01)

Gil de Paz, A., Silich, S. A., Madore, B. F., Sánchez Contreras, C., Zamorano, J. \& Gallego, J. 2002, ApJ, 573, L101

Gil de Paz, A., Zamorano, J. \& Gallego, J. 1999, MNRAS, 306, 975

Hidalgo-Gámez, A. M., Masegosa, J., Olofsson, K. 2002, RMxAC, 12, 242

Hunter, D. A., Elmegreen, B. G. \& Baker, A. L. 1998, ApJ, 493, 595

Hunter, D.A. 2002: The Outer Edges of Dwarf Irregular Galaxies, Online Proceedings (http: / / www. lowell.edu), D. Hunter and S. Oey (eds.)

Koprolin, W. 2002: The Outer Edges of Dwarf Irregular Galaxies, Online Proceedings (http: / /www. lowell.edu), D. Hunter and S. Oey (eds.)

Loose, H. \& Fricke, K. 1980, Astrophys. Letters, 21, 65

Loose, H. H., Krügel, E. \& Tutukov, A. 1982, A\&A, 105, 342

Loose, H. H. \& Thuan, T. X. 1986, Star Forming Dwarf Galaxies and Related Objects, 73 (LT86)

Makarova, L. N., Karachentsev, I. D., Grebel, E. K., Barsunova, O. Yu. 2002, A\&A, 384, 72

Marlowe, A. T., Meurer, G. R., Heckman, T. M. 1999, ApJ, 522, 183

Marlowe, A. T., Meurer, G. R., Heckman, T. M., Schommer, R. 1997, ApJS, 112, 285

Méndez, D.I., Esteban, C. 1999, AJ, 118, 2723

Meurer, G. R., Staveley-Smith, L. \& Killeen, N. E. B. 1998, MNRAS, 300, 705

Näslund, M. \& Jörsäter, S. 1997, A\&A, 325, 915

Noeske, K. G. 1999, Diploma Thesis, University of Göttingen

Noeske, K. G., Guseva, N. G., Fricke, K. J., Izotov, Y. I., Papaderos, P., Thuan, T. X. 2000, A\&A, 361, 31 (Paper IV)

Noeske, K.G., Papaderos, P., Cairós, L.M. \& Fricke, K.J. 2003, A\&A, submitted (N03a) (Paper I)

Noeske, K.G., Papaderos, P., Cairós, L.M. \& Fricke, K.J. 2003, A\&A, submitted (N03b) (Paper II)

O’Connell, R. W., Gallagher, J. S. \& Hunter, D. A. 1994, ApJ, 433, 65

Papaderos, P. 1998, PhD Thesis, University of Göttingen

Papaderos, P., Fricke, K. J., Thuan, T. X., Izotov, Y. I., Nicklas, H. 1999, A\&A, 352, L57

Papaderos, P., Izotov, Yu.I., Thuan, T.X., Noeske, K.G., Guseva, N.G., Fricke, K.J. 2002,

A\&A,

Papaderos, P., Loose, H.-H., Fricke, K. J., Thuan, T. X. 1996b, A\&A,314, 59 (P96b)

Papaderos, P., Loose, H.-H., Thuan, T.X., Fricke, K.J., 1996a, A\&AS 120, 207 (P96a)

Parodi, B. R., Barazza, F. D. \& Binggeli, B. 2002, A\&A, 388, 29 
Patterson, R. J., Thuan, T. X. 1996, ApJS, 107, 103

Pohlen, M., Dettmar, R-J., Lütticke, R. \& Aronica, G. 2002a, A\&A, 392, 807

Pohlen, M., Dettmar, R-J., Beckman, J. \& Balcells, M. 2002: The Outer Edges of Dwarf Irregular Galaxies, Online Proceedings (http: / / www. lowel l . edu), D. Hunter and S. Oey (eds.)

Ryder, S. D. \& Dopita, M. A. 1994, ApJ, 430, 142

Salzer, J. J. \& Norton, S. A. 1999, ASP Conf. Ser. 170: The Low Surface Brightness Universe, 253

Schulz, J., Fritze - v. Alvensleben, U., Möller, C. S., Fricke, K. J. 2002, A\&A, in press

Simpson, C. E. \& Gottesman, S. T. 2000, AJ, 120, 2975

Sung, E., Han, C., Ryden, B. S., Chun, M. \& Kim, H. 1998, ApJ, 499, 140

Telles, E., Melnick, J., \& Terlevich, R. 1997, MNRAS, 288, 78

Tifft, W. G. 1982, ApJS, 50, 319

van Zee, L. 2000, AJ, 119, 2757

van Zee, L., Salzer, J. J. \& Skillman, E. D. 2001, AJ, 122, 121

van Zee, L., Skillman, E.D. \& Salzer, J.J. 1998, AJ, 116, 1186

Vennik, J., Hopp, U., \& Popescu, C. C. 2000, A\&AS, 142, 399

Walter, F., Brinks, E., Duric, N., \& Klein, U. 1997, AJ, 113, 2031

Whitmore, B. C., Lucas, R. A., McElroy, D. B., Steiman-Cameron, T. Y., Sackett, P. D. \&

Olling, R. P. 1990, AJ, 100, 1489

Worthey, G. 1994, ApJS, 95, 107 


\title{
The Cometary Blue Compact Dwarf Galaxies Mkn 59 and Mkn 71: ${ }^{0}$
}

\section{A Clue to Dwarf Galaxy Evolution?}

\author{
K.G. Noeske ${ }^{1}$, N.G. Guseva ${ }^{2}$, K.J. Fricke ${ }^{1}$, Y.I. Izotov ${ }^{2}$, P. Papaderos ${ }^{1}$, T.X. Thuan ${ }^{3}$ \\ ${ }^{1}$ Universitäts-Sternwarte, Geismarlandstraße 11, D-37083 Göttingen, Germany \\ ${ }^{2}$ Main Astronomical Observatory of National Academy of Sciences of Ukraine, Goloseevo, \\ $252650 \mathrm{Kiev}-22$, Ukraine \\ ${ }^{3}$ Astronomy Department, University of Virginia, Charlottesville, VA 22903, USA
}

Summary. "Cometary" Blue Compact Dwarf Galaxies (iI,C BCDs) are characterized by an off-center starburst close to the end of their elongated stellar bodies. This rare phenomenon may carry some clues on how collective star formation ignites and propagates in gas-rich lowmass stellar systems. This off-center burst may be a fortuitous enhancement of the otherwise moderate star-forming activity of a dwarf irregular (dI), or may be caused by a set of special properties of such systems or their environment. We attempt here a first investigation of this issue by analysing two prototypical examples of cometary dwarf galaxies, the nearby iI,C BCDs Markarian 59 and Markarian 71, both containing an extraordinarily luminous $\mathrm{H}$ II region in the outskirts of a dI-like host. Using deep ground-based spectrophotometric data ${ }^{1,2}$ and HST images ${ }^{3}$, we study the physical state of the starburst regions and the structural properties of the underlying irregular galaxies. We find that the average metallicities show small scatter in the vicinity of the star-forming regions and along the major axis of Mkn 59 which suggests that mixing of heavy elements must have been efficient on scales of several kpc. The azimuthally averaged radial intensity distributions of the underlying host galaxies in either iI,C BCD can be approximated by an exponential law with a central surface brightness and scale length that is intermediate between typical $\mathrm{iE} / \mathrm{nE}$ BCDs and dwarf irregulars. Spectral population synthesis models in combination with colour magnitude diagrams and colour profiles yield a most probable formation age of $\sim 2 \mathrm{Gyr}$ for the low surface brightness (LSB) host galaxies in both iI,C BCDs, with upper age limits of $\sim 4$ Gyr for Mkn 59 and $\sim 3$ Gyr for Mkn 71, i.e. significantly lower than the typical age of several Gyr derived for the LSB component of $\mathrm{iE} / \mathrm{nE}$ BCDs. These findings raise the question whether iI,C systems form a distinct physical class within

\footnotetext{
${ }^{0}$ This paper has been published in this form in Astronomy \& Astrophysics, Volume 361, p. 33

${ }^{1}$ Obtained at the German-Spanish Astronomical Center, Calar Alto, operated by the MaxPlanck-Institute for Astronomy, Heidelberg, jointly with the Spanish National Commission for Astronomy.

2 Obtained at the Kitt Peak National Observatory, National Optical Astronomy Observatories, operated by the Association of Universities for Research in Astronomy, Inc., under cooperative agreement with the National Science Foundation.

${ }^{3}$ Based on observations with the NASA/ESA Hubble Space Telescope, obtained at the Space Telescope Science Institute, which is operated by AURA, INC., under NASA contract No. NAS 5-26555.
} 
BCDs with respect to the age and structural properties of their hosts, or whether they represent an evolutionary stage connecting young i0 BCDs and "classical" iE/nE BCDs. In spite of the scarcity of available data, a review of the properties of analogous objects studied in the local universe and at medium redshifts provides some support for this evolutionary hypothesis.

\section{IV.1 Introduction}

Despite the effort that has been devoted to the investigation of Blue Compact Dwarf Galaxies (BCDs), the origin of their recurrent starburst activity as well as its impact on their spectrophotometric and dynamical evolution is still poorly understood. A thorough investigation of these processes is required before questions pertaining to evolutionary connections among dwarf irregulars (dIs), dwarf ellipticals (dEs) and BCDs (Thuan 1985, Davies \& Phillipps 1988, Papaderos et al. 1996b; hereafter P96b, Patterson \& Thuan 1996, Salzer \& Norton 1998, Marlowe et al. 1997, 1999) can be answered. Deep imaging of the low surface brightness (LSB) component of BCDs, discovered first by Loose \& Thuan (1985; hereafter LT85), disclosed an evolved stellar population underlying the regions of active star formation (SF) (Loose \& Thuan 1986, Kunth et al. 1988, Papaderos et al. 1996a; hereafter P96a). Therefore, the initial hypothesis that BCDs are "extragalactic H II regions" lacking an older stellar population (Sargent \& Searle 1970) had to be dismissed for the majority of these systems.

An exception to this finding is made by a tiny fraction $(\lesssim 1 \%)$ of young galaxy candidates which recently have been identified among BCDs (Thuan et al. 1997, Papaderos et al. 1998, Thuan \& Izotov 1999). There is increasing observational evidence that such systems, invariably found among the most metal-deficient $\left(Z \lesssim 1 / 20 Z_{\odot}\right.$, Thuan \& Izotov 1999, Izotov \& Thuan 1999) members of the BCD class, have started forming the bulk of their stellar component less than $100 \mathrm{Myr}$ ago. This qualifies them as local counterparts of primeval galaxies, i.e. the first galactic building blocks which are thought to have formed at high redshifts.

It seems meaningful to search among the variety of dwarf galaxies for possible successors of young galaxies, i.e. objects in a more evolved stage with a substantial fraction of their masses still in gaseous form. An age sequence built therefrom would allow to study the evolution of nearby dwarf galaxies, which may at the same time give clues to early galaxy evolution processes at high redshift. This is of great interest for the assessment of the starburst-driven evolution of young galaxies, some of which may become the building blocks of larger systems and for the understanding of issues like the faint blue galaxy excess at intermediate redshifts (Babul \& Rees 1992, Koo et al. 1997, Guzmán et al. 1998).

Loose \& Thuan (1985) developed a morphological classification scheme for BCDs and found that $\sim 90 \%$ of their sample is made up of $\mathrm{iE}-$ and $\mathrm{nE}-$ objects. These show, respectively, an irregular or nuclear starburst component superposed on a smooth elliptical LSB host galaxy with optical colours pointing to ages of several Gyr (Loose \& Thuan 1986, P96b, Telles \& Terlevich 1997, Noeske 1999). In contrast, the few members of the rare class of i0 BCDs examined so far have been found to be young galaxy candidates (Thuan et al. 1997, Papaderos et al. 1998, Thuan \& Izotov 1999, Izotov et 
al. 1999), as they apparently lack a smooth stellar underlying component that would have required previous episodes of SF to be built.

Only the class of iI BCDs, characterized by both irregular LSB components and starburst regions, has not yet been studied systematically. This paper focuses on the subset of iI,C BCDs (LT85) where "C" denotes a cometary appearance caused by a bright star-forming complex at one end of an elongated stellar galactic body (see Figs. IV.1 and IV.6). In the notation of Salzer et al. (1989) an iI,C BCD is to be included in the class of H II hotspot galaxies; these systems seem to systematically differ from other subclasses of $\mathrm{H}$ II galaxies with respect to the metallicities and excitation properties of their ionized gas. While the main bodies of iI,C systems are reminiscent of low surface

Table IV.1. Ground-based imaging

\begin{tabular}{|c|c|c|c|c|c|}
\hline \multirow{3}{*}{$\begin{array}{l}\text { Object } \\
\alpha(2000.0) \\
\delta(2000.0) \\
(1)\end{array}$} & \multirow{3}{*}{$\begin{array}{l}\text { Filter } \\
\text { (2) }\end{array}$} & \multicolumn{4}{|c|}{$\begin{array}{l}t_{\exp } \text { Night } \\
\text { sec }\end{array}$} \\
\hline & & & & & \\
\hline & & (3) & (4) & (5) & (6) \\
\hline \multirow[t]{2}{*}{ Mkn 59} & $\bar{B}$ & 180 & 4 & 1.67 & 22.4 \\
\hline & $B$ & 1100 & 3 & 2.95 & 22.5 \\
\hline 125900.3 & $R$ & 540 & 4 & 1.57 & 20.8 \\
\hline+345043 & $I$ & 300 & 4 & 1.45 & 18.8 \\
\hline \multirow[t]{2}{*}{$\overline{\text { Mkn } 71}$} & $\bar{B}$ & 1500 & 1 & 1.59 & 22.1 \\
\hline & $R$ & 300 & 4 & 1.48 & 20.6 \\
\hline 72841.4 & $R$ & 600 & 3 & 2.92 & 20.7 \\
\hline \multirow[t]{3}{*}{+691126} & $I$ & 600 & 1 & 1.67 & 18.9 \\
\hline & $\mathrm{H} \alpha$ & 900 & 3 & 3.07 & - \\
\hline & $\mathrm{H} \alpha$ & 900 & 4 & 1.74 & - \\
\hline
\end{tabular}

brightness dwarf irregulars (dIs) or magellanic irregulars (Kennicutt et al. 1980, Dottori et al. 1994, Wilcots et al. 1996) with some widespread low-level SF, the bright offcenter H II regions are typical for BCDs with respect to their sizes, $\mathrm{H} \alpha$ luminosities and electron temperatures. The detection of Super Star Clusters (SSCs) in these spots points to a very recent or still ongoing starburst episode (Kennicutt et al. 1980, Dottori et al. 1994, Barth et al. 1994).

Optical surveys of BCDs (LT85, Kunth et al. 1988, Salzer et al. 1989, P96a, Marlowe et al. 1997, Doublier et al. 1997) and dIs (e.g. Hunter et al. 1998) suggest that in these systems SF per unit area correlates with the local surface density of the underlying host galaxy. In this respect, the occurrence of a starburst with the amplitude observed in iI,C BCDs at one end of an elongated stellar LSB body appears puzzling and may be interpreted in at least two ways:

(i) iI,C BCDs are in fact dIs or magellanic irregulars caught during a brief stochastic enhancement of their otherwise moderate star formation rate per unit area. This would imply that both the structural properties of their stellar hosts and H I envelopes will be indistinguishable from those of other dIs. 


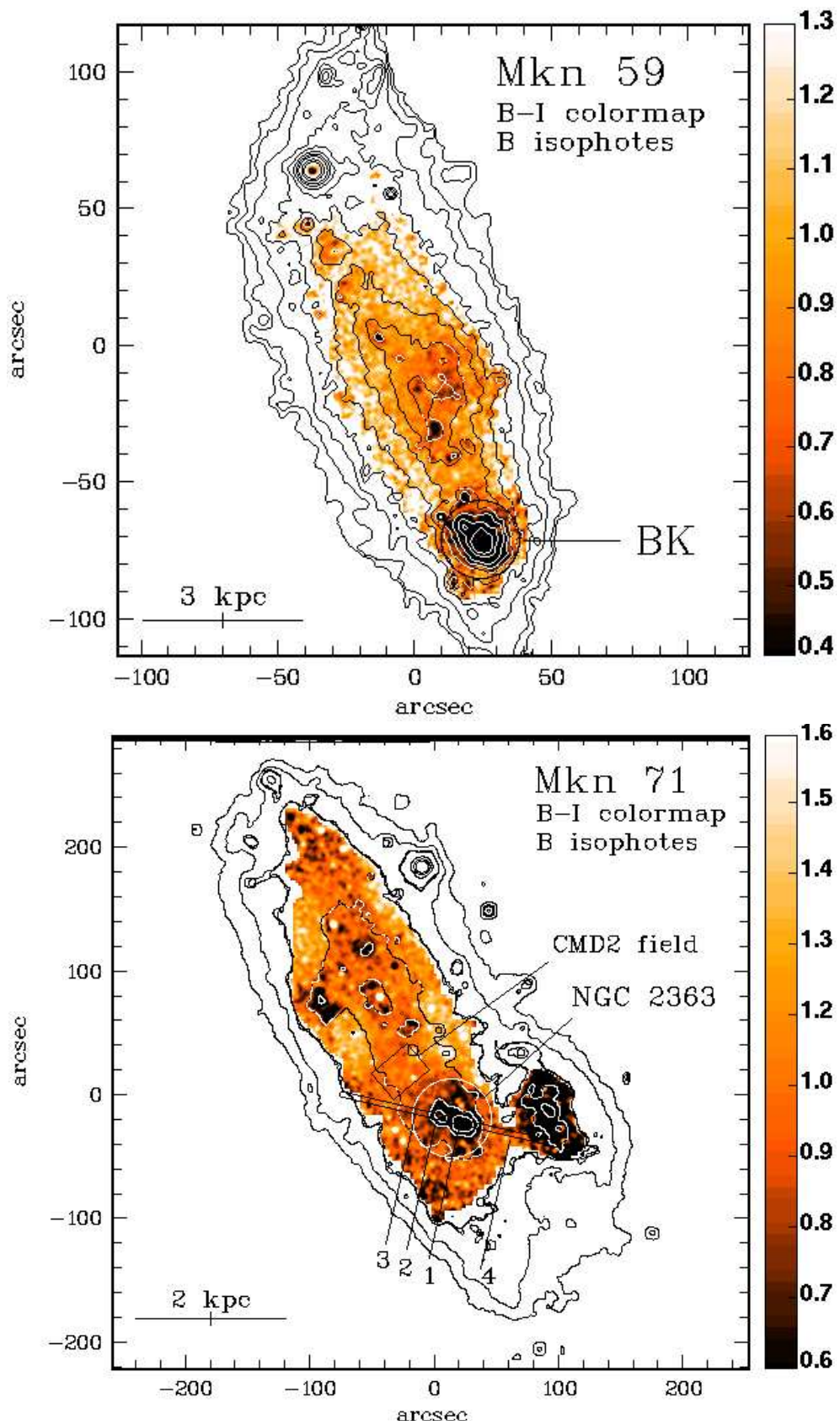

Fig. IV.1. $(B-I)$ colour maps with overplotted $B$ band isophotes. top Mkn 59; the isophotes correspond to surface brightness levels of 20,21, 22 and $22.5-25.5 \mathrm{mag} / \square^{\prime \prime}$ in steps of $0.5 \mathrm{mag}$. The bright starburst knot (BK), described in detail by Dottori et al. (1994) is indicated. bottom Mkn 71; the isophotes correspond to surface brightness levels of 21, 22, 23, 24, 24.5 and 25 mag/ $\square^{\prime \prime}$. The giant H II complex NGC 2363, as well as the field used to derive the colourmagnitude diagram of the galaxy's underlying stellar population from HST data (CMD 2) are marked. The indices 1-4 along the orientation of the long-slit (centered at the axis origin) mark the regions from which the spectra displayed in Figure 8 were extracted. North is up and east to the left. 
(ii) In iI,C BCDs the conditions necessary for the ignition of a starburst are not fulfilled at the center but in the outskirts of their LSB components. This may be due to a conspiracy of intrinsic and external properties of an evolved BCD (kinematics of the gaseous component and Dark Matter distribution or external perturbation by a companion or intracluster gas) or alternatively be a common feature among less evolved systems.

Here we attempt to test such hypotheses by analysing optical data of two nearby iI,C BCDs, Mkn 59 (NGC 4861) and Mkn 71 (NGC 2366, hosting the H II region complex NGC 2363). The distance to Mkn 71 was determined to $D=3.44 \mathrm{Mpc}$ through observations of Cepheids (Tolstoy et al. 1995). For Mkn 59 different models for infall and small-scale perturbations in the Virgo Cluster environment yield a distance between $\sim 0.7 \mathrm{Mpc}$ (Heckman et al. 1998) and 17.8 Mpc (Tully 1988). The principal results of this work do not depend on the exact distance to Mkn 59 (cf. Section IV.3.1). Throughout this paper, we adopt $D=10.7 \mathrm{Mpc}$ (Heckman et al. 1998).

Using broad- and narrow band images and long-slit spectra from own groundbased observations and archival HST data, we shall investigate the physical state and the chemical composition of the ISM and examine whether the iI,C BCDs under study differ from typical $\mathrm{iE} / \mathrm{nE} \mathrm{BCDs}$ with respect to the ages and structural properties of their LSB hosts. In Section 2, we describe our observations and data analysis. We present our results in Section 3, and discuss them in Section 4. In Section 5, we summarize our results and conclusions.

\section{IV.2 Data acquisition and processing}

\section{IV.2.1 Ground-based imaging}

\section{IV.2.1.1 Observations and data reduction}

Images were taken on March 7th - 10th 1997 at the 2.2m telescope of the GermanSpanish Astronomical Center, Calar Alto, Spain. We used the Calar Alto Faint Object Spectrograph (CAFOS), equipped with a $2048 \times 2048$ pixel SITe\#1d CCD. The focal ratio of $\mathrm{f} / 4.4$ in the $\mathrm{RC}$ focus and the pixel size of $24 \mu \mathrm{m} /$ pixel yield an instrumental scale of $0.53^{\prime \prime} /$ pixel and a usable field of view of $\sim 15^{\prime}$. At a gain ratio of $2.3 \mathrm{e}^{-} \mathrm{ADU}^{-1}$ the read-out noise was $<3$ counts (rms). Column (1) of the observing log (Table IV.1) contains the equatorial coordinates of the targets, cols. (2) and (3) give the filters and exposure times, respectively. Column (4) lists the night of the observing run, starting with night 1 from March 7th to March 8th 1997. The seeing and the mean sky surface brightness during each exposure are given in cols. (5) and (6). During each night, dark-, bias-, and flat-field exposures were taken, and the photometric standard field NGC 2419 (Christian et al. 1985) was observed at different zenith angles. Using the ESO MIDAS ${ }^{4}$ software package, standard reduction and calibration steps were applied to the raw images. During nights 3 and 4 the conditions were photometric, resulting in

\footnotetext{
${ }^{4}$ Munich Image Data Analysis System, provided by the European Southern Observatory (ESO).
} 


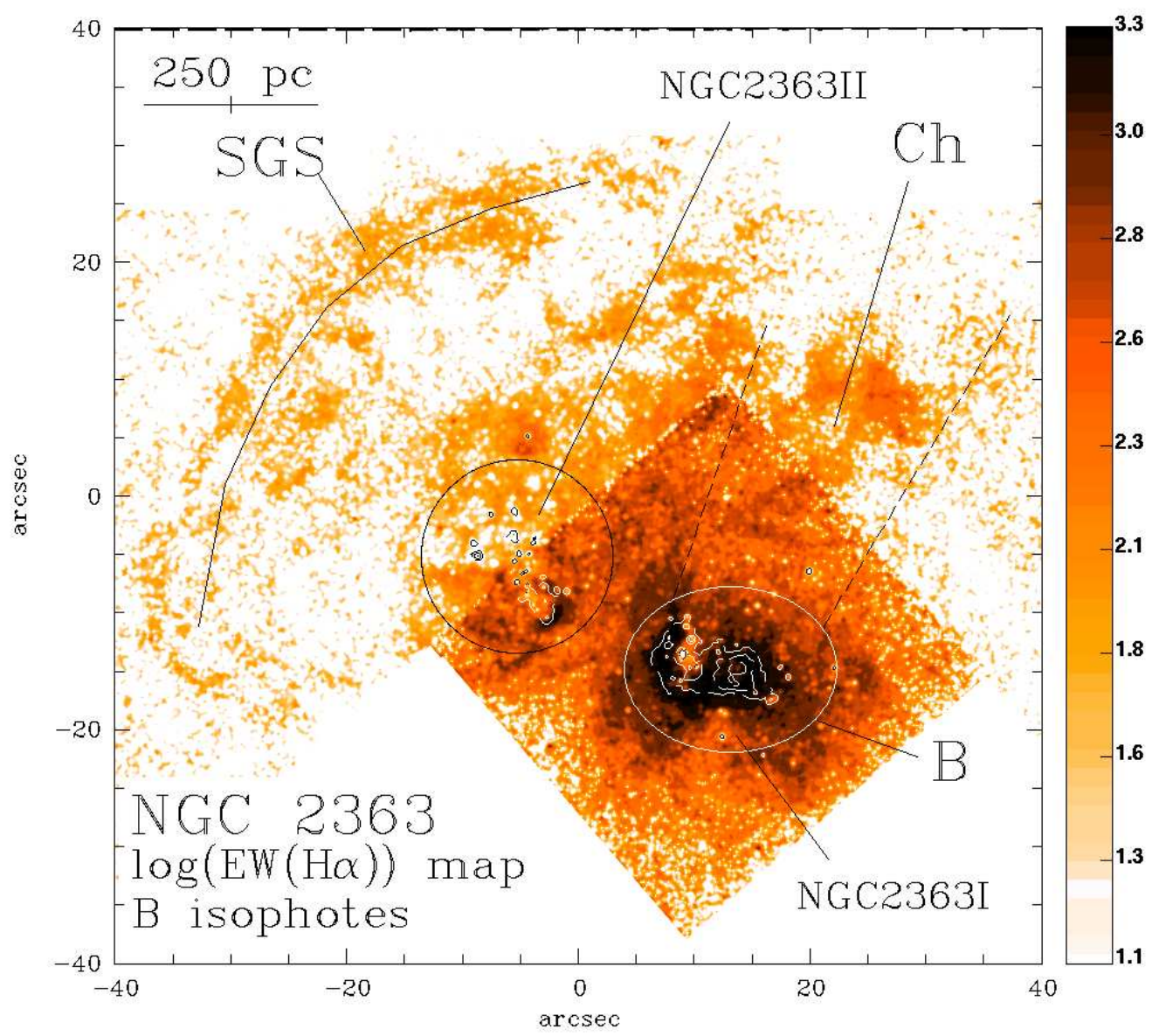

Fig. IV.2. Logarithmic representation of the $\mathrm{H} \alpha$ equivalent width $(E W(\mathrm{H} \alpha)[\AA])$ map of the H II complex NGC 2363 within Mkn 71, computed from HST/WFPC2 images as described in Section 2.1.1. $B$ band contours at $18-20 \mathrm{mag} / \square^{\prime \prime}$ mark the positions of the young star clusters NGC 2363 I and II within NGC 2363. The arc-like structure, extending out to $\sim 0.5 \mathrm{kpc}$ northeast of NGC 2363 II, probably marks a supergiant shell (SGS) (Hunter \& Gallagher 1997). The expanding superbubble (B) and the probable outflow chimney (Ch, delimited by the pair of dashed lines) around NGC 2363 I (Roy et al. 1991) are indicated. The spatial distribution of the young stellar background in NGC 2363 I and NGC 2363 II is reviewed in Section IV.4.1. North is up and east to the left.

calibration errors well below 0.05 mag. A poor atmospheric transparency during night 1 led to strong airmass-dependent terms for the $B$ band exposure of Mkn 71. Therefore the calibration was accomplished by comparing aperture photometry of bright isolated point sources on HST- and ground-based images. The photometric uncertainty of the latter $B$ band exposure was found to be $\lesssim 0.1 \mathrm{mag}$. All exposures for one object were aligned to each other using positions of point sources; the transformed images were generated by re-sampling the original image using a flux-conserving routine. Colour maps were derived after the resolution of different frames had been equalized by con- 
volving the exposure with the better resolution with a normalized gaussian distribution of adequate width.

The continuum level in the $\mathrm{H} \alpha$ images was inferred by scaling the $R$ band images by an empirically determined factor $C$ so that the fluxes of field stars were matched between the raw $\mathrm{H} \alpha$ - and the scaled $R$ exposures. In turn, the scaled $R$ band exposures were subtracted from the raw $\mathrm{H} \alpha$ images, giving the emission flux $F_{\mathrm{em}}$ at each pixel. The latter approach is strictly correct only when the $\mathrm{H} \alpha$ emission line is contributing a minor fraction of the line-of-sight $R$ band flux. In regions where $\mathrm{H} \alpha$ emission makes a substantial fraction of the photons received in the $R$ band the empirical scaling may lead to a slight overestimation of the continuum flux. In these cases the correct $\mathrm{H} \alpha$ emission $F_{e m}^{c}$ is obtained from $F_{e m}$ as:

$$
F_{e m}^{c}=\frac{1}{1-C \cdot T_{R}(\mathrm{H} \alpha)} F_{e m}
$$

where $C$ is the empirical factor described above and $T_{R}(\mathrm{H} \alpha)$ the mean transmission of the $R$ band filter at the $\mathrm{H} \alpha$ wavelength. The resulting narrow band frames were calibrated using continuum-subtracted $\mathrm{H} \alpha$ exposures of the planetary nebula NGC 2392 for which aperture flux measurements are given by Kaler (1983).

To derive $\mathrm{H} \alpha$ equivalent width maps we subtracted first the corrected line emission frame $F_{e m}^{c}$ (Eq.IV.1) from the raw $\mathrm{H} \alpha$ image to obtain a continuum image $F_{\text {cont }}$, which was then normalized to the continuum flux per $1 \AA$ wavelength interval by dividing it by the effective width of the $\mathrm{H} \alpha$ filter. The emission line image $F_{e m}^{c}$ was divided by the transmission of the $\mathrm{H} \alpha$ filter at the $\mathrm{H} \alpha$ wavelength. The resulting frame, containing the total $\mathrm{H} \alpha$ emission flux, was divided by the $1 \AA$ - normalized continuum flux frame to obtain the $\mathrm{H} \alpha$ equivalent width image.

The colour- and $\mathrm{H} \alpha$ maps were corrected for interstellar extinction following Savage \& Mathis (1979) and using the extinction coefficients $C(\mathrm{H} \beta)$ given in Section IV.2.3, which translate into $E(B-V)=0.06 \mathrm{mag}$ and $E(B-V)=0.08 \mathrm{mag}$ for Mkn 59 and Mkn 71, respectively.

\section{IV.2.1.2 Surface photometry}

Surface brightness profiles (SBPs) are the result of a transformation of a galaxy's 2dimensional flux pattern into a monotonically decreasing 1-dimensional intensity distribution. For a set of methods to compute the photometric radius $R^{\star}(\mu)$, i.e. the radius of a circle with an area equal to the one enclosed by the isophote at the surface brightness level $\mu\left[\mathrm{mag} / \square^{\prime \prime}\right]$, see e.g. Loose \& Thuan (1986) and P96a. The method of summing up the area defined by pixels with fluxes exceeding a threshold $I(\mu)$ (method iii; P96a) was considered most appropriate for the BCDs analyzed here as both their underlying and starburst components are of irregular morphology. Other techniques, such as isophote integration or ellipse fitting, though well suited for more regular BCDs, are hardly applicable to the optical images of Mkn 59 and Mkn 71, mainly due to the fact that their high surface brightness components split into many star-forming regions occupying a substantial fraction of the host galaxy's surface. 

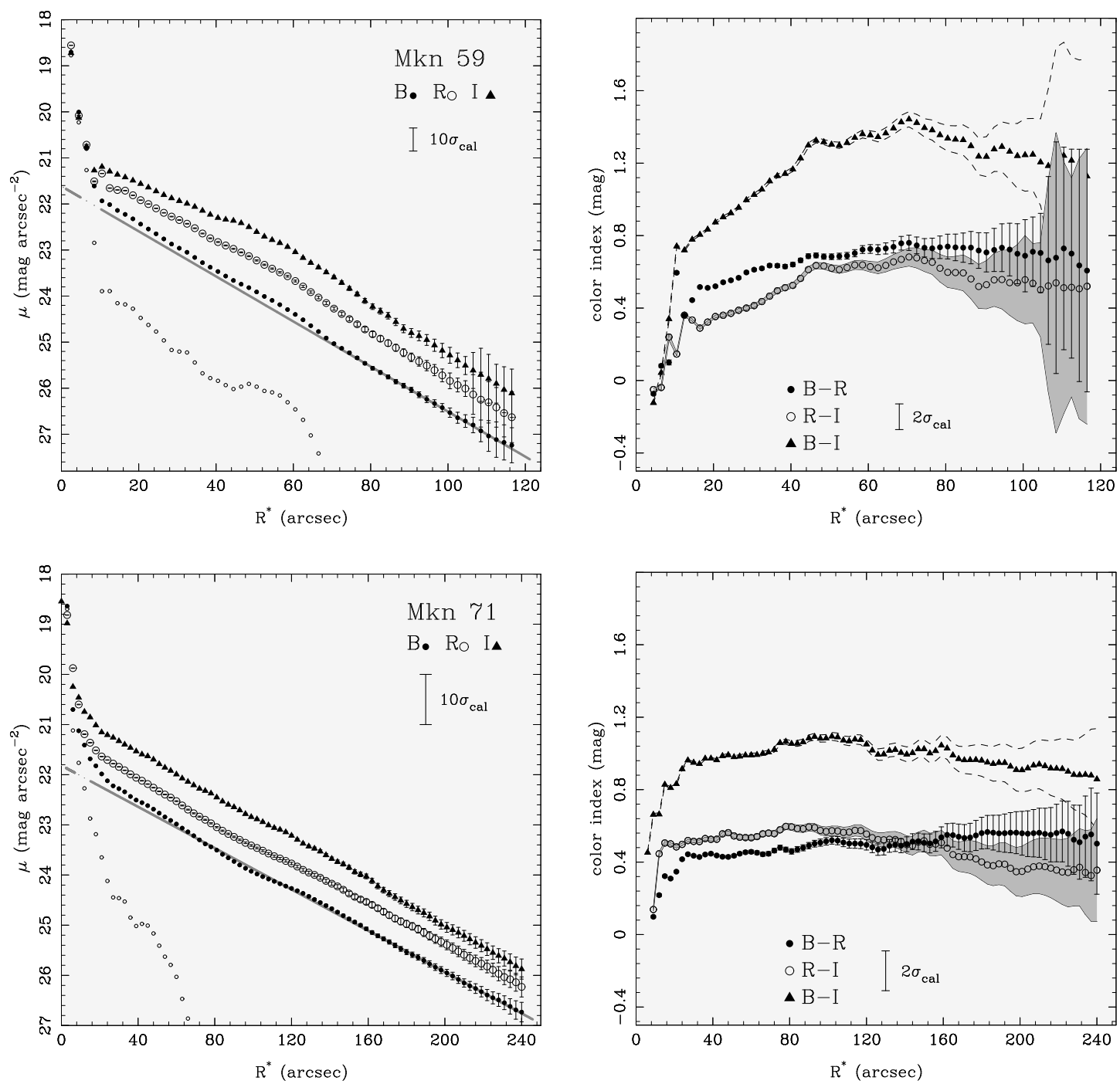

Fig. IV.3. left Surface brightness profiles of Mkn 59 (top) and Mkn 71 (bottom) in $B, R$ and $I$. Small circles show the surface brightness distribution of the residual $B$ band emission in excess of the exponential fit to the LSB component (straight line). The calibration uncertainty $\sigma_{c a l}$ effecting a vertical shift of the entire profile is displayed 10 times enlarged. right Radially averaged colour profiles with 2 times enlarged calibration error bars. Note that for surface brightness levels fainter than $\sim 23.5 B$ mag $\operatorname{arcsec}^{-2}$ the colour index of the underlying LSB galaxy becomes nearly constant.

For each surface brightness level $\mu$, the number $N(\mu)$ of all pixels within a polygonal aperture with a count level $\geq I(\mu)$ was determined; the corresponding photometric radius is

$$
R^{\star}(\mu)=\frac{1}{\pi} \sqrt{N(\mu) \cdot A_{\mathrm{pxl}}},
$$

where $A_{\mathrm{pxl}}$ is the solid angle per pixel in $\square^{\prime \prime}$. Numerical simulations showed that for the technique described above Poisson photon noise may cause a systematic flattening of SBPs at a signal to noise $S / N$ level $\lesssim 10$. This frequently observed artificial profile 
Table IV.2. Structural properties of the starburst- and LSB components of Mkn 59 and Mkn 71 derived from profile decomposition.

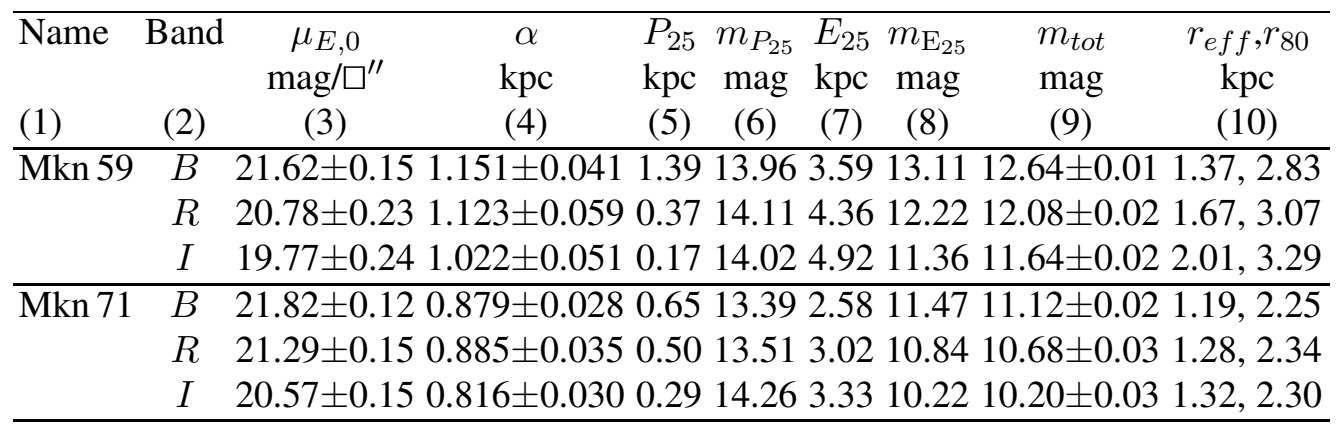

flattening could be, however, satisfactorily corrected down to a $(S / N)$ level of $\approx 1$ by using the adaptive filtering algorithm implemented in MIDAS (see Richter et al. 1991 for a description). The uncertainties at each photometric radius were calculated taking into account the corresponding intensity, the sky noise of each image, and the number of pixels involved in its calculation (cf. P96a). Colour profiles were computed by subtracting SBPs from each other; the surface brightness- and colour profiles displayed in Figure 3 are corrected for interstellar extinction (cf. Section IV.2.1.1).

Unlike stellar systems like globular clusters and giant ellipticals where the mass to light $(M / L)$ ratio may be regarded nearly constant over the whole system, the integrated luminosity of BCDs originates from two distinct stellar populations with substantially different ages and $M / L$ ratios. Although the LSB component contributes on average one half of the total $B$ band luminosity of a BCD (P96b, Salzer \& Norton 1998), it contains most of the stellar mass of the system. Therefore, its intensity distribution yields information on the inner gravitational potential within which the starburst ignites and evolves. In order to disentangle the light distribution of the older host galaxy from the one of the superimposed starburst component, we applied a simple 2-component decomposition scheme adjusted interactively to each profile, instead of iteratively fitting the full nonlinear 3-component scheme described in P96a. The intensity distribution of the LSB component is approximated by an exponential fitting law of the form

$$
I_{\mathrm{E}}\left(R^{\star}\right)=I_{\mathrm{E}, 0} \exp \left(-\frac{R^{\star}}{\alpha}\right)
$$

or equivalently

$$
\mu\left(R^{\star}\right)=\mu_{\mathrm{E}, 0}+1.086\left(\frac{R^{\star}}{\alpha}\right)
$$

where $\alpha$ denotes its exponential scale length in arcsec and $\mu$ the surface brightness level in mag/ $\square^{\prime \prime}$. Equation (IV.4) was adjusted to each profile by applying an error weighted linear fit to the data, at radii sufficiently large to be free from the contamination of the starburst light, i.e. where the colour profiles become constant and $\mathrm{H} \alpha$ 
emission vanishes. To check at which surface brightness levels gaseous emission becomes negligible, we overlaid the $\mathrm{H} \alpha$ emission-and $E W(\mathrm{H} \alpha)$ maps with broad band isophotes (cf. Figs. IV.1 and IV.2) . The fits, obtained at radii $R^{\star} \geq 76^{\prime \prime}$ for Mkn 59 and $R^{\star} \geq 160^{\prime \prime}$ for Mkn 71, yield the extrapolated central surface brightness $\mu_{E, 0}$ and the exponential scale length $\alpha$. The radial surface brightness distribution of the starburst component can be computed from the residual luminosity in excess of the fit Eq. (IV.4).

Table IV.2 summarizes the results of the profile decomposition. In the same way as described in P96a, cols. (3) and (4) give the central surface brightness and exponential scale length of the LSB component. Columns (5) and (7) list respectively the isophotal radii of the starburst component $P_{25}$ (practically the "plateau radius" used in P96a), and of the stellar LSB host, $E_{25}$. Both radii were determined from extinction-corrected SBPs at a surface brightness level of $25 \mathrm{mag} / \square^{\prime \prime}$. Columns (6) and (8) contain the apparent magnitudes of the latter components determined within $P_{25}$ and $E_{25}$, respectively, and col. (9) the total apparent magnitude in each band as derived from integration of the corresponding SBP out to the last measured point. Column (10) lists the effective radius $r_{e f f}$ and the radius $r_{80}$, enclosing $80 \%$ of the galaxy's total flux.

\section{IV.2.2 HST images}

\section{IV.2.2.1 Data reduction}

Next we shall discuss colour-magnitude diagrams (CMDs) of the H II complex NGC 2363 and the main body of Mkn 71 derived from Hubble Space Telescope (HST) archival data. The following analysis is based on integrations of $2 \times 800$ and $1 \times 700 \mathrm{sec}$ in the F439W filter, $2 \times 800 \mathrm{sec}$ in the F547M filter and $1 \times 900$ plus $1 \times 600 \mathrm{sec}$ in the F656N filter taken on January 8th, 1996 with the WFPC2 (PI: Drissen, Proposal ID 06096). All images were reduced through the standard pipeline as described in Holtzman et al. (1995a). Exposures taken in the same filter were co-added and corrected for cosmic ray events using the STSDAS package and IRAF ${ }^{2}$. We applied a charge transfer efficiency correction to the data as described in the HST Data Handbook (1998), performed a sky background subtraction and used the synthetic zero points and transformation coefficients given by Holtzman et al. (1995b) to transform the measured fluxes to magnitudes in the Johnson $U B V R I$ system. For the $\mathrm{H} \alpha$ narrow band filter, F656N, the continuum was subtracted as described in Section IV.2.1.1; the correction given by Eq. (IV.1) was not necessary, as the $\mathrm{H} \alpha$ line lies outside the wavelength range covered by the F547M filter. The calibration of the $\mathrm{H} \alpha$ images was done following the prescriptions by Holtzman et al. (1995b).

\section{IV.2.2.2 Colour-magnitude diagrams}

The HII region NGC 2363 within Mkn 71 (Figure 1; right) was centered on the PC/WF1 chip (instrumental scale of 0 '.046/pixel), allowing the resolution of compact

\footnotetext{
${ }^{2}$ IRAF is distributed by the National Optical Astronomy Observatories, which is operated by the Association of Universities for Research in Astronomy, Inc., under cooperative agreement with the National Science Foundation.
} 

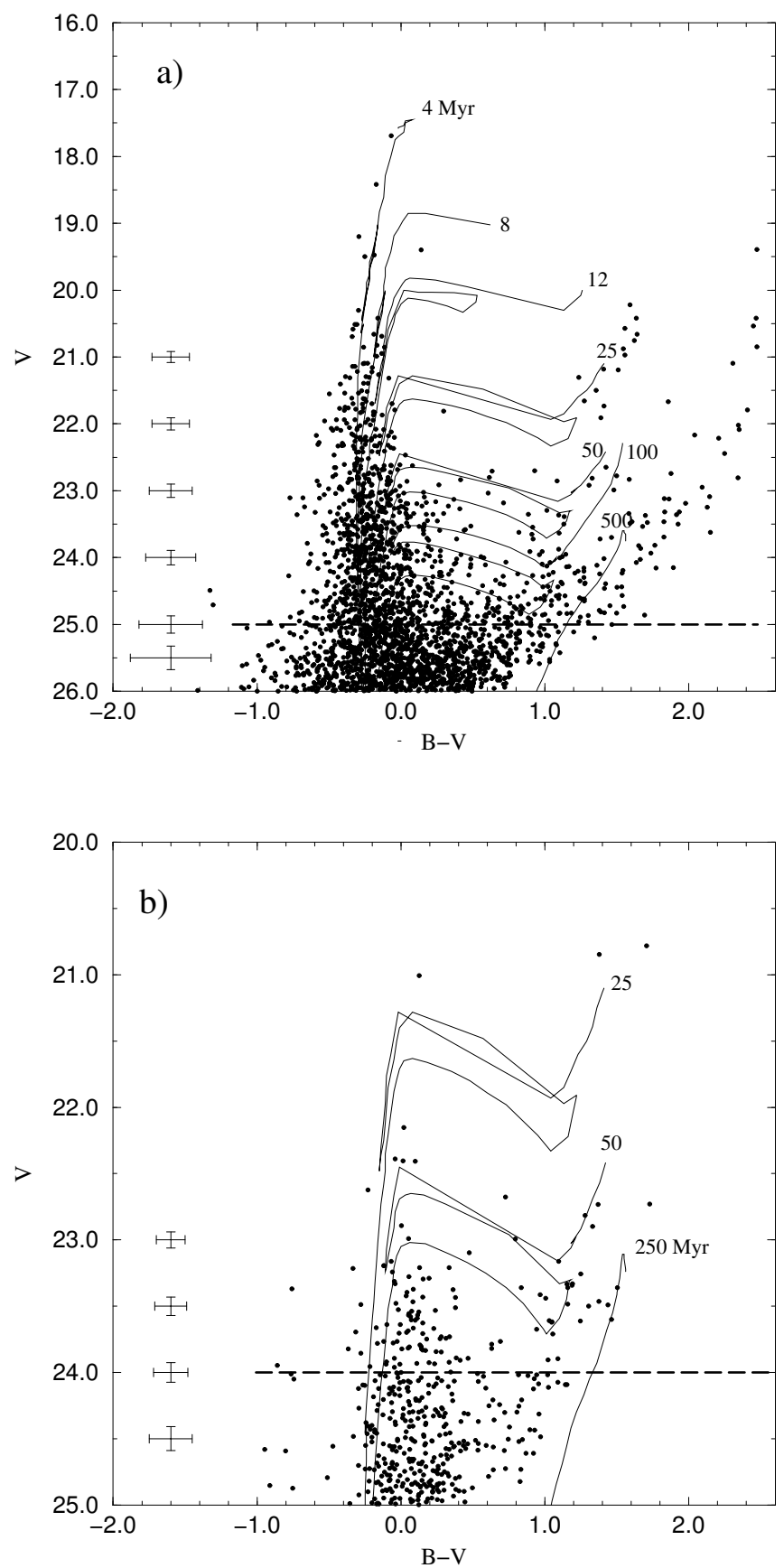

Fig. IV.4. $V$ vs. $(B-V)$ colour-magnitude diagrams (CMDs) of Mkn 71; overplotted are isochrones by Bertelli et al. (1994) for a metallicity of $1 / 20 Z_{\odot}$. Crosses to the left illustrate the mean errors in both axes as a function of the apparent $V$ magnitude; the dashed lines indicate the estimated confidence limit for the $B$ and $V$ band. a CMD derived within the PC1 chip of WFPC2, centered on the H II region NGC 2363; the isochrones correspond to ages of 4, 8, 12, 25, 50, 100 and 500 Myr. b CMD of the region of the main body of Mkn 71 indicated in Figure 1 (right panel, see label "CMD 2") where active star-forming regions are absent as derived from WF4 data. The isochrones correspond to ages of 25, 50 and $250 \mathrm{Myr}$. 
groups of stars with a mean linear separation of $\sim 0.8 \mathrm{pc}$. We utilized the PC1 data to derive CMDs in order to further constrain the results of the spectral population synthesis analysis (Section 3.3) using the DAOPHOT II stellar photometry package under ESO MIDAS. A model for the point spread function (PSF), necessary for performing multiple-PSF fitting in crowded fields, was computed from several isolated bright stars in each frame. Because of the severe crowding within some regions of NGC 2363 as well as local background variations due to strong gaseous emission, we evaluated the photometric uncertainties and completeness limits of the resulting point-source identification files, taking into account the error growth towards fainter luminosities and the magnitude histograms (cf. Figure IV.5).

The measured fluxes were calibrated and transformed to the Johnson $B$ and $V$ filters as described in the previous section. The luminosity functions of the detected point sources (Figure IV.5, left panel) suggest a reasonable completeness for sources brighter than $\approx 26$ mag in both $B$ and $V$. Faintwards of the latter magnitude cutoff the photometric errors become dominant (see Figure IV.4). Close to the detection limit, an additional error source is introduced by the small-scale variations of the background due to gaseous emission as well as strong noise peaks which possibly are misinterpreted as faint stars (cf. the excess counts at low luminosities in the upper left panel of Figure IV.5). We shall therefore consider the data reliable down to a limit of $\sim 25$ mag in $B$ and $V$, close to the one Drissen et al. (1999) obtain for the same data set. This cutoff is sufficient for our purposes; from a simple comparison of data points and isochrones it becomes evident that, given the photometric errors and poor time resolution of the $(B-V)$ CMD, no firm conclusions on the SF history can be drawn for ages $\gtrsim 50$ Myr. A second CMD was derived in the same way for a region on the WF4 chip apparently free of any appreciable signatures of current SF (marked in Figure IV.1) to obtain information on the galaxy's underlying stellar population separately. From an inspection of the right panels of Figure IV.5, and from the considerations referring to the CMD of NGC 2363 we estimate the confidence limit to $\approx 24$ mag for both $B$ and $V$. Either CMD is shown in Figure IV.4 with typical photometric uncertainties for different magnitude intervals along with synthetic isochrones for a metallicity of $Z=0.001$ adopted from Bertelli et al. (1994).

\section{IV.2.3 Spectroscopic observations and data reduction}

Spectrophotometric observations of Mkn 59 and Mkn 71 were obtained on March 17th and 18th 1994, using the Ritchey-Chrétien spectrograph of the KPNO 4m Mayall telescope. We used a $2^{\prime \prime} \times 300^{\prime \prime}$ slit with the KPC-10A grating $\left(316\right.$ lines $\left.\mathrm{mm}^{-1}\right)$ in first order, with a GG 385 order separation filter which cuts off all second order contamination for wavelengths blueward of $7400 \AA$. This instrumental setup gave a spatial scale along the slit of 0.69 pixel $^{-1}$, a scale perpendicular to the slit of $2.7 \AA$ pixel $^{-1}$, a spectral range of 3500-7500 $\AA$, and a spectral resolution of $\sim 5 \AA$. The seeing was $1^{\prime \prime} .5$ FWHM. Mkn 59 was observed at two slit positions (cf. Figure IV.6), both centered at the brightest star-forming region with P.A. $=59^{\circ}$ and P.A. $=15^{\circ}$ (close to the direction of the major axis). The total exposure time for the first orientation of the slit was 70 
Table IV.3. Emission Line Intensities in Mkn 59

\begin{tabular}{|c|c|c|c|}
\hline \multirow[t]{2}{*}{$\overline{\text { Ion }}$} & region 1 & \multicolumn{2}{|c|}{ region 2} \\
\hline & $F(\lambda) / F(\mathrm{H} \beta)$ & $F(\lambda) / F(\mathrm{H} \beta)$ & $I(\lambda) / I(\mathrm{H} \beta)$ \\
\hline 3727 [O II] & $1.0086 \pm 0.0018 \quad 1.0879 \pm 0.0020$ & $1.9191 \pm 0.0832$ & $2.0801 \pm 0.1049$ \\
\hline $3750 \mathrm{H} 12$ & $0.0204 \pm 0.00060 .0268 \pm 0.0009$ & $\ldots$ & ... \\
\hline $3770 \mathrm{H} 11$ & $0.0280 \pm 0.00060 .0349 \pm 0.0009$ & $\ldots$ & ... \\
\hline 3798 H10 & $0.0341 \pm 0.00060 .0415 \pm 0.0009$ & $\ldots$ & $\ldots$ \\
\hline 3835 H9 & $0.0498 \pm 0.00060 .0581 \pm 0.0009$ & $\ldots$ & $\ldots$ \\
\hline 3868 [Ne III] & $0.4566 \pm 0.00110 .4872 \pm 0.0012$ & $0.3179 \pm 0.0503$ & $0.3366 \pm 0.0584$ \\
\hline $3889 \mathrm{He} \mathrm{I}+\mathrm{H} 8$ & $0.1633 \pm 0.00080 .1786 \pm 0.0010$ & $\ldots$ & $\ldots$ \\
\hline $3968[\mathrm{Ne} \mathrm{III}]+\mathrm{H} 7$ & $0.2806 \pm 0.00090 .3014 \pm 0.0011$ & $0.1467 \pm 0.0309$ & $0.2848 \pm 0.0698$ \\
\hline $4026 \mathrm{He} \mathrm{I}$ & $0.0110 \pm 0.00050 .0116 \pm 0.0005$ & $\ldots$ & $\ldots$ \\
\hline 4069 [S II] & $0.0097 \pm 0.00060 .0102 \pm 0.0006$ & $\ldots$ & $\ldots$ \\
\hline 4076 [S II] & $0.0031 \pm 0.00360 .0033 \pm 0.0038$ & $\ldots$ & $\ldots$ \\
\hline $4101 \mathrm{H} \delta$ & $0.2396 \pm 0.00080 .2552 \pm 0.0010$ & $0.1296 \pm 0.0246$ & $0.2606 \pm 0.0598$ \\
\hline $4340 \mathrm{H} \gamma$ & $0.4589 \pm 0.00110 .4766 \pm 0.0012$ & $0.3775 \pm 0.0316$ & $0.4737 \pm 0.0461$ \\
\hline 4363 [O III] & $0.0890 \pm 0.00060 .0916 \pm 0.0006$ & $0.0560 \pm 0.0326$ & $0.0550 \pm 0.0349$ \\
\hline $4471 \mathrm{He} \mathrm{I}$ & $0.0357 \pm 0.00050 .0365 \pm 0.0005$ & $0.0875 \pm 0.0320$ & $0.0846 \pm 0.0339$ \\
\hline 4658 [ III] & $0.0054 \pm 0.00040 .0054 \pm 0.0004$ & $\ldots$ & $\ldots$ \\
\hline 4686 He II & $0.0131 \pm 0.00050 .0132 \pm 0.0005$ & $\ldots$ & $\cdots$ \\
\hline $4713[$ Ar IV] + He I & $0.0084 \pm 0.00040 .0085 \pm 0.0004$ & $\cdots$ & $\ldots$ \\
\hline 4740 [Ar IV] & $0.0055 \pm 0.00040 .0056 \pm 0.0004$ & $\ldots$ & $\ldots$ \\
\hline $4861 \mathrm{H} \beta$ & $1.0000 \pm 0.0017 \quad 1.0000 \pm 0.0017$ & $1.0000 \pm 0.0495$ & $1.0000 \pm 0.0554$ \\
\hline $4921 \mathrm{He} \mathrm{I}$ & $0.0082 \pm 0.00040 .0081 \pm 0.0004$ & $\ldots$ & ... \\
\hline 4959 [O III] & $2.0670 \pm 0.00302 .0503 \pm 0.0030$ & $1.4993 \pm 0.0672$ & $1.3588 \pm 0.0667$ \\
\hline $5007[\mathrm{O} \mathrm{III}]$ & $6.1585 \pm 0.00786 .0917 \pm 0.0078$ & $4.3242 \pm 0.1668$ & $3.8956 \pm 0.1645$ \\
\hline $5199[\mathrm{~N} \mathrm{I}]$ & $0.0033 \pm 0.00040 .0033 \pm 0.0004$ & $\ldots$ & $\ldots$ \\
\hline $5271[\mathrm{Fe} \mathrm{III]}$ & $0.0019 \pm 0.00040 .0019 \pm 0.0003$ & $\ldots$ & $\ldots$ \\
\hline 5518 [Cl III] & $0.0043 \pm 0.00040 .0041 \pm 0.0003$ & $\ldots$ & $\ldots$ \\
\hline 5538 [Cl III $]$ & $0.0026 \pm 0.00040 .0025 \pm 0.0004$ & $\ldots$ & $\ldots$ \\
\hline $5876 \mathrm{He} \mathrm{I}$ & $0.1138 \pm 0.00050 .1075 \pm 0.0005$ & $0.0854 \pm 0.0160$ & $0.0698 \pm 0.0144$ \\
\hline $6300[\mathrm{O}$ I] & $0.0210 \pm 0.00040 .0195 \pm 0.0004$ & $\ldots$ & $\ldots$ \\
\hline 6312 [S III] & $0.0209 \pm 0.00040 .0194 \pm 0.0003$ & $\ldots$ & $\cdots$ \\
\hline 6364 & $=0.00030 .006$ & $\ldots$ & $\ldots$ \\
\hline 6548 [N II] & $0.0242 \pm 0.00050 .0222 \pm 0.0005$ & $\ldots$ & . \\
\hline $6563 \mathrm{H} \alpha$ & $3.0749 \pm 0.00412 .8214 \pm 0.0041$ & $3.6134 \pm 0.1384$ & $2.8062 \pm 0.1277$ \\
\hline 6583 [N II] & $0.0540 \pm 0.00040 .0495 \pm 0.0004$ & $0.1800 \pm 0.0229$ & $0.1377 \pm 0.0194$ \\
\hline $6678 \mathrm{He} \mathrm{I}$ & $0.0323 \pm 0.00040 .0295 \pm 0.0003$ & $0.0427 \pm 0.0137$ & $0.0324 \pm 0.0114$ \\
\hline 6717 [S II] & $0.1052 \pm 0.00050 .0959 \pm 0.0005$ & $0.2983 \pm 0.0266$ & $0.2258 \pm 0.0225$ \\
\hline $6731[\mathrm{~S} \mathrm{II}]$ & $0.0785 \pm 0.00050 .0715 \pm 0.0004$ & $0.1597 \pm 0.0245$ & $0.1207 \pm 0.0204$ \\
\hline $7065 \mathrm{He} \mathrm{I}$ & $0.0280 \pm 0.0003 \quad 0.0252 \pm 0.0003$ & $\ldots$ & $\ldots$ \\
\hline 7136 [Ar III] & $0.0862 \pm 0.00050 .0774 \pm 0.0004$ & $0.1331 \pm 0.0178$ & $0.0974 \pm 0.0144$ \\
\hline $7281 \mathrm{He} \mathrm{I}$ & $0.0051 \pm 0.0003 \quad 0.0046 \pm 0.0002$ & $\ldots$ & $\ldots$ \\
\hline 7320 [O II] & $0.0186 \pm 0.00040 .0166 \pm 0.0004$ & ... & $\ldots$ \\
\hline 7330 [O II $]$ & $0.0209 \pm 0.00050 .0187 \pm 0.0005$ & ... & $\cdots$ \\
\hline$C\left(\mathrm{H}_{1}\right.$ & $0.110 \pm 0.002$ & 0.23 & $=0.050$ \\
\hline$F(\mathrm{H} \beta)^{a}$ & $15.08 \pm 0.02$ & $0.28 \pm$ & $=0.01$ \\
\hline$E W(\mathrm{H} \beta) \AA$ & $150.1 \pm 0.2$ & & \pm 1 \\
\hline$E W(\mathrm{abs}) \AA$ & $0.35 \pm 0.04$ & $3.9 \pm$ & \\
\hline
\end{tabular}

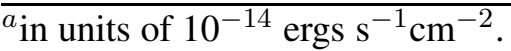



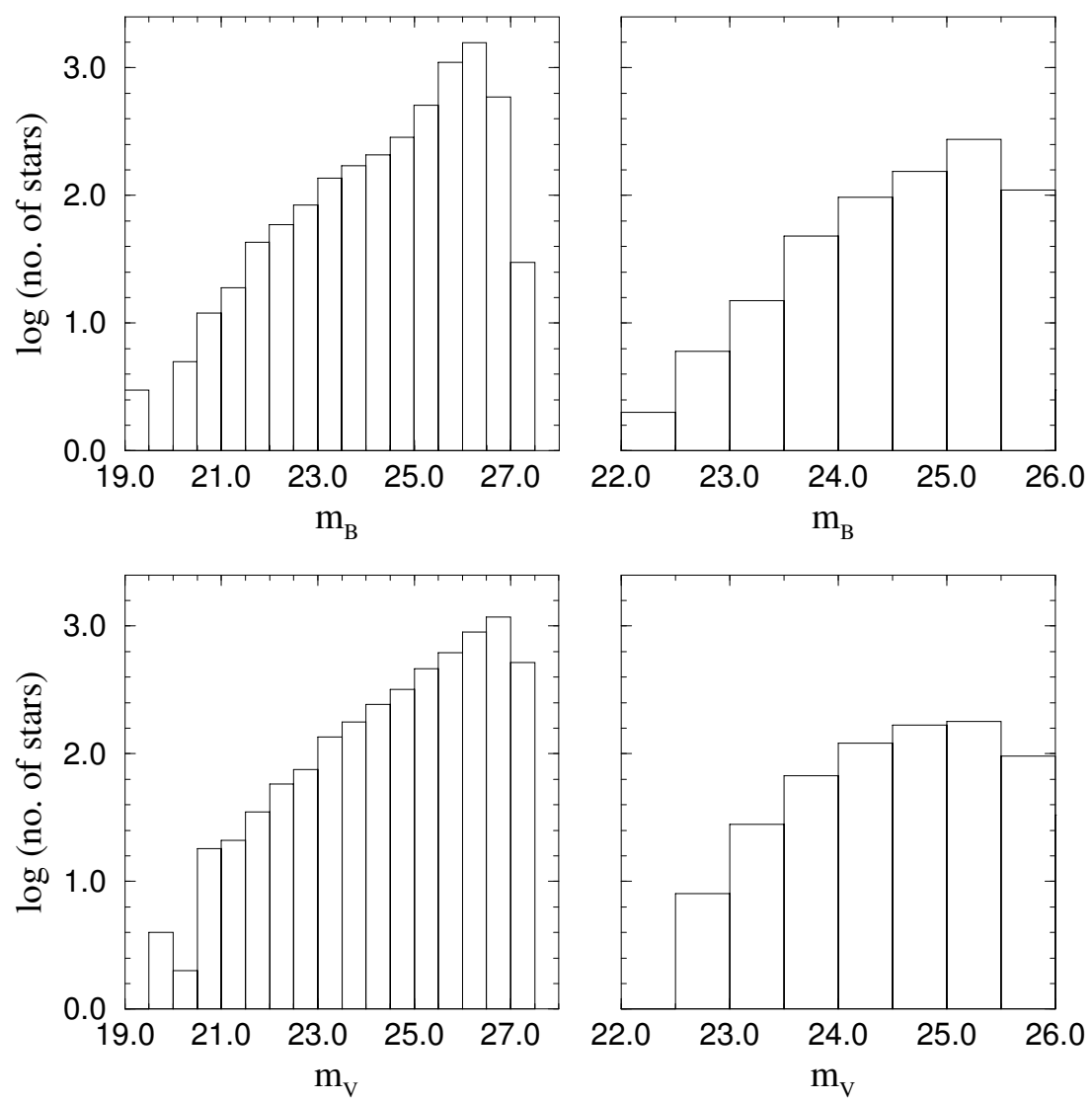

Fig. IV.5. Luminosity functions of the point sources included in the colour-magnitude diagrams (Figure IV.4) as obtained within the PC1- (left) and WF4 chip (right) of HST WFPC2.

minutes and was broken up into four subexposures. At the second slit orientation a single 15 minutes exposure was taken.

Mkn 71 was observed at a slit orientation of P.A. $=77^{\circ}$ (see Figure IV.1), centered on the brightest star-forming region (NGC 2363 I). The total exposure time was 29 minutes, broken up into five subexposures. Three Kitt Peak IRS spectroscopic standard stars were observed during each night for flux calibration. The airmasses during all observations were $\leq 1.25$, therefore a correction for atmospheric dispersion was not necessary. Spectra of He-Ne-Ar comparison lamps were obtained before and after each observation to calibrate the wavelength scale.

The data reduction was done with the IRAF software package. The twodimensional spectra were bias-subtracted and flat-field corrected. Then the IDENTIFY, REIDENTIFY, FITCOORD, TRANSFORM, BACKGROUND and CALIBRATE routines were used to perform the wavelength calibration, correction for distortion and tilt, night sky subtraction and flux calibration for each frame. Onedimensional spectra were extracted from the flux-calibrated two-dimensional spectra using the IRAF task APALL. For Mkn 59 we chose ten $7^{\prime \prime} \times 2^{\prime \prime}$ regions along the major 
Table IV.4. Emission Line Intensities in Mkn 59

\begin{tabular}{|c|c|c|}
\hline \multirow[t]{2}{*}{ Ion } & \multicolumn{2}{|c|}{ region 3} \\
\hline & $F(\lambda) / F(\mathrm{H} \beta)$ & $I(\lambda) / I(\mathrm{H} \beta)$ \\
\hline 3727 [O II] & $3.1730 \pm 0.1866$ & $2.9505 \pm 0.2018$ \\
\hline 3868 [Ne III] & $0.5371 \pm 0.0516$ & $0.4994 \pm 0.0528$ \\
\hline $3968[\mathrm{Ne} \mathrm{III}]+\mathrm{H} 7$ & $0.2177 \pm 0.0343$ & $0.2832 \pm 0.0603$ \\
\hline $4101 \mathrm{H} \delta$ & $0.1568 \pm 0.0426$ & $0.2377 \pm 0.0836$ \\
\hline $4340 \mathrm{H} \gamma$ & $0.4335 \pm 0.0518$ & $0.4833 \pm 0.0714$ \\
\hline 4363 [O III] & $0.0594 \pm 0.0287$ & $0.0552 \pm 0.0287$ \\
\hline $4861 \mathrm{H} \beta$ & $1.0000 \pm 0.0739$ & $1.0000 \pm 0.0848$ \\
\hline 4959 [O III] & $1.3522 \pm 0.0939$ & $1.2574 \pm 0.0939$ \\
\hline 5007 [O III] & $3.8327 \pm 0.2218$ & $3.5640 \pm 0.2219$ \\
\hline $6300[\mathrm{O} \mathrm{I}]$ & $\ldots$ & $\ldots$ \\
\hline $6563 \mathrm{H} \alpha$ & $2.5651 \pm 0.1519$ & $2.4329 \pm 0.1693$ \\
\hline 6583 [N II] & $0.2177 \pm 0.0374$ & $0.2024 \pm 0.0378$ \\
\hline 6717 [S II] & $0.3142 \pm 0.0410$ & $0.2921 \pm 0.0418$ \\
\hline 6731 [S II] & $0.3037 \pm 0.0430$ & $0.2824 \pm 0.0437$ \\
\hline 7136 [Ar III] & ... & .. \\
\hline$C(\mathrm{H} \beta) \operatorname{dex}$ & \multicolumn{2}{|c|}{$0.000 \pm 0.077$} \\
\hline$F(\mathrm{H} \beta)^{a}$ & \multicolumn{2}{|c|}{$0.21 \pm 0.01$} \\
\hline$E W(\mathrm{H} \beta) \AA$ & \multicolumn{2}{|c|}{$51.1 \pm 2.7$} \\
\hline$E W(\mathrm{abs}) \AA$ & \multicolumn{2}{|c|}{$3.85 \pm 1.49$} \\
\hline
\end{tabular}

axis of the galaxy (i.e the slit at P.A. $=15^{\circ}$ ), the locations of which are indicated in Figure IV.6. Six of these spectra are shown in Figure IV.7. From the slit at P.A. $=59^{\circ}$ the subspectra were extracted at each pixel row along the slit, so that the resulting areas were $00^{\prime \prime} 69 \times 2^{\prime \prime}$ each. For Mkn 71 (see Figure IV.1), the subspectra from regions 1 and 2 were extracted from $7^{\prime \prime} \times 2^{\prime \prime}$ regions, while for regions 3 and 4 areas of $14^{\prime \prime} \times 2^{\prime \prime}$ and $28^{\prime \prime} \times 2^{\prime \prime}$ were used to obtain a sufficiently high $S / N$. The extracted spectra are shown in Figure IV.8.

The emission line intensities were measured utilizing a Gaussian profile fitting. All spectra were corrected for interstellar extinction, where the extinction coefficient $C(\mathrm{H} \beta)$ was derived from the hydrogen Balmer decrement using the equations given in Izotov et al. (1994) and the theoretical hydrogen emission line flux ratios from Brocklehurst (1971). The variations of $C(\mathrm{H} \beta)$ along different regions (Tables IV.3 and IV.4) arise mainly from observational uncertainties (imperfect focussing at the blue and red ends of the spectra), and from different combinations of Balmer lines that could be reliably measured in each subspectrum to determine the local extinction. While each spectrum was corrected using its individual value of $C(\mathrm{H} \beta)$, images were extinctioncorrected adopting a uniform value of $C(\mathrm{H} \beta)=0.09$ and $C(\mathrm{H} \beta)=0.12$ for Mkn 59 and Mkn 71, respectively (Guseva et al. 2000). 


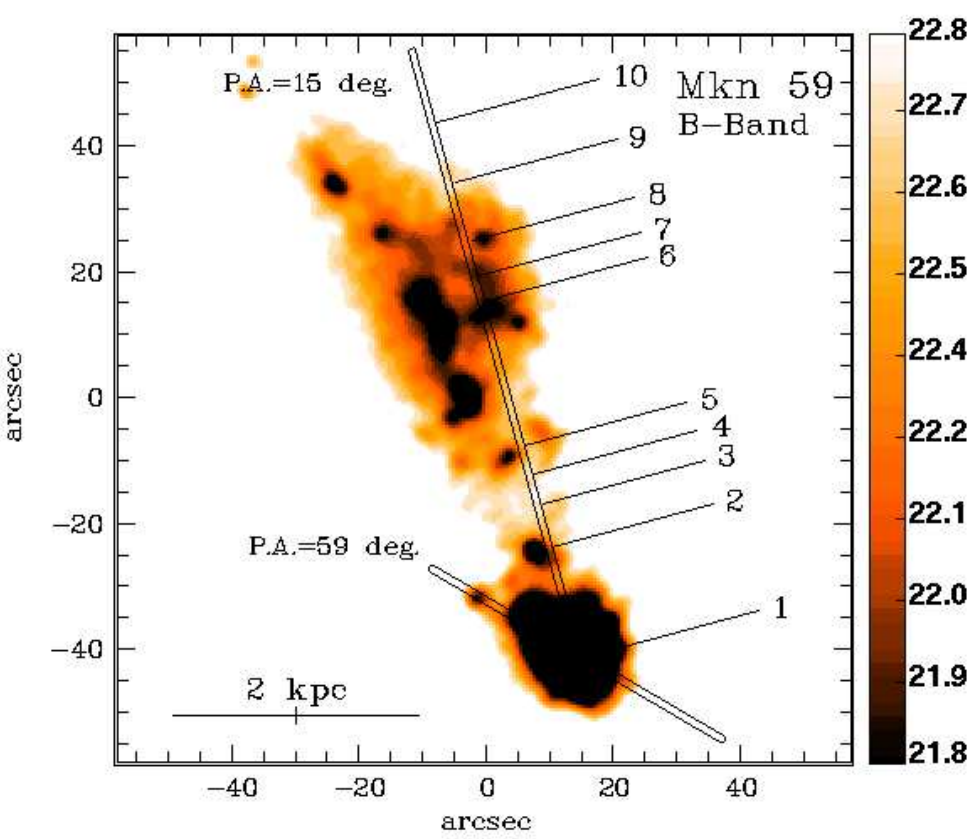

Fig. IV.6. $B$ band image of Mkn 59; the two different slit positions and the regions investigated individually (see Section IV.2.3) are shown. The grey scale to the right displays the surface brightness levels in B mag $\operatorname{arcsec}^{-2}$.

The electron temperature $T_{e}(\mathrm{O}$ III $)$ was derived from the observed flux ratio $[\mathrm{O}$ III $](\lambda 4959+\lambda 5007) / \lambda 4363$. In cases where the [O III] $\lambda 4363$ emission line was not detected, we used the so-called upper branch of the Edmunds \& Pagel (1984) calibration of the total oxygen emission line flux $[\mathrm{O}$ II $] \lambda 3727+[\mathrm{O}$ III $](\lambda 4959+\lambda 5007)$ vs. the electron temperature to determine $T_{e}$ and derived the oxygen abundance following van Zee et al. (1998a).

The observed $(F(\lambda))$ and corrected $(I(\lambda))$ emission line fluxes relative to the $\mathrm{H} \beta$ emission line fluxes for 3 regions in Mkn 59 (slit P.A. $=15^{\circ}$ ) are listed in Tables IV.3 and IV.4. The tables contain only the regions where the emission line [O III] $\lambda 4363$ was detected at a $S / N$ that allowed for a reliable flux measurement. Also listed are the extinction coefficient $C(\mathrm{H} \beta)$, the observed flux of the $\mathrm{H} \beta$ emission line and its equivalent width $E W(\mathrm{H} \beta)$ along with the equivalent width of hydrogen absorption lines $E W$ (abs). The line intensities of the brightest regions (1 and 2) of Mkn 71 are presented in Izotov et al. (1997).

Applying the electron temperature $T_{e}\left(\mathrm{O}\right.$ III), ionic abundances of $\mathrm{O}^{2+}, \mathrm{Ne}^{2+}$ and $\mathrm{Ar}^{3+}$ were derived. The temperature $T_{e}(\mathrm{O}$ II $)$ was inferred, according to Izotov et al. (1994, 1996), from the relation between $T_{e}(\mathrm{O}$ II $)$ and $T_{e}(\mathrm{O}$ III) using $\mathrm{H}$ II region photoionization models by Stasińska (1990). From $T_{e}(\mathrm{O}$ II $)$, the $\mathrm{O}^{+}$and $\mathrm{N}^{+}$ionic abundances were determined, while the intermediate value of the electron temperature $T_{e}$ (S III) served to derive the ionic abundances of $\mathrm{Ar}^{2+}$ and $\mathrm{S}^{2+}$ (Garnett 1992). The [S II] $\lambda 6717 / \lambda 6731$ ratio was used to determine the electron number density $N_{e}(\mathrm{~S}$ II). Total heavy element abundances were derived after correcting for unseen stages of ionization following Izotov et al. (1994, 1997) and Thuan et al. (1995). 
Table IV.5. Heavy Element Abundances in Mkn 59

\begin{tabular}{|c|c|c|c|}
\hline Property & region 1 & region 2 & region 3 \\
\hline$T_{e}(\mathrm{O}$ III $)(\mathrm{K})$ & $13,469 \pm 38$ & $13,069 \pm 3,299$ & $13,558 \pm 2,910$ \\
\hline$T_{e}(\mathrm{O}$ II $)(\mathrm{K})$ & $12,979 \pm 34$ & $12,762 \pm 3,008$ & $13,027 \pm 2,626$ \\
\hline$T_{e}(\mathrm{~S}$ III $)(\mathrm{K})$ & $13,224 \pm 32$ & $12,916 \pm 2,738$ & $13,293 \pm 2,415$ \\
\hline$N_{e}(\mathrm{~S} \mathrm{II})\left(\mathrm{cm}^{-3}\right)$ & $79 \pm 10$ & $10 \pm 10$ & $536 \pm 561$ \\
\hline $\mathrm{O}^{+} / \mathrm{H}^{+}\left(\times 10^{5}\right)$ & $1.502 \pm 0.012$ & $3.001 \pm 2.164$ & $4.239 \pm 2.571$ \\
\hline $\mathrm{O}^{++} / \mathrm{H}^{+}\left(\times 10^{5}\right)$ & $8.757 \pm 0.069$ & $6.152 \pm 4.365$ & $5.095 \pm 3.013$ \\
\hline $\mathrm{O}^{+3} / \mathrm{H}^{+}\left(\times 10^{5}\right)$ & $\ldots$ & $\ldots$ & $\ldots$ \\
\hline $\mathrm{O} / \mathrm{H}\left(\times 10^{5}\right)$ & $10.26 \pm 0.070$ & $9.154 \pm 4.872$ & $9.334 \pm 3.961$ \\
\hline $12+\log (\mathrm{O} / \mathrm{H})$ & $8.011 \pm 0.003$ & $7.962 \pm 0.231$ & $7.970 \pm 0.184$ \\
\hline$[\mathrm{O} / \mathrm{H}]^{a}$ & $-0.919 \pm 0.003$ & $-0.968 \pm 0.231$ & $-0.960 \pm 0.184$ \\
\hline $\mathrm{N}^{+} / \mathrm{H}^{+}\left(\times 10^{7}\right)$ & $4.902 \pm 0.040$ & $14.26 \pm 10.28$ & $19.98 \pm 12.12$ \\
\hline $\operatorname{ICF}(\mathrm{N})$ & 6.83 & 3.05 & 2.20 \\
\hline $\log (\mathrm{N} / \mathrm{O})$ & $-1.486 \pm 0.006$ & $-1.323 \pm 0.550$ & $-1.327 \pm 0.455$ \\
\hline $\mathrm{Ne}^{++} / \mathrm{H}^{+}\left(\times 10^{5}\right)$ & $1.639 \pm 0.014$ & $1.243 \pm 0.931$ & $1.646 \pm 1.016$ \\
\hline $\mathrm{ICF}(\mathrm{Ne})$ & 1.17 & 1.49 & 1.83 \\
\hline $\log (\mathrm{Ne} / \mathrm{O})$ & $-0.728 \pm 0.006$ & $-0.695 \pm 0.461$ & $-0.491 \pm 0.374$ \\
\hline $\mathrm{S}^{+} / \mathrm{H}^{+}\left(\times 10^{7}\right)$ & $2.164 \pm 0.013$ & $4.634 \pm 1.900$ & $7.776 \pm 2.782$ \\
\hline $\mathrm{S}^{++} / \mathrm{H}^{+}\left(\times 10^{7}\right)$ & $14.85 \pm 0.281$ & $\ldots$ & $\ldots$ \\
\hline $\mathrm{ICF}(\mathrm{S})$ & 1.89 & $\ldots$ & $\ldots$ \\
\hline $\log (\mathrm{S} / \mathrm{O})$ & $-1.504 \pm 0.006$ & $\cdots$ & ... \\
\hline $\mathrm{Ar}^{++} / \mathrm{H}^{+}\left(\times 10^{7}\right)$ & $3.497 \pm 0.023$ & $4.652 \pm 1.738$ & $\ldots$ \\
\hline $\mathrm{Ar}^{+3} / \mathrm{H}^{+}\left(\times 10^{7}\right)$ & $1.049 \pm 0.080$ & ; & $\ldots$ \\
\hline $\mathrm{ICF}(\mathrm{Ar})$ & 1.02 & 1.53 & $\ldots$ \\
\hline $\log (\mathrm{Ar} / \mathrm{O})$ & $-2.346 \pm 0.008$ & $-2.108 \pm 0.266$ & $\ldots$ \\
\hline $\mathrm{Fe}^{++} / \mathrm{H}^{+}\left(\times 10^{7}\right)$ & $1.505 \pm 0.123$ & $\ldots$ & $\ldots$ \\
\hline $\mathrm{ICF}(\mathrm{Fe})$ & 8.54 & $\ldots$ & $\ldots$ \\
\hline $\log (\mathrm{Fe} / \mathrm{O})$ & $-1.902 \pm 0.012$ & $\ldots$ & $\ldots$ \\
\hline
\end{tabular}

The resulting ionic and heavy element abundances for the $3 \mathrm{H}$ II regions in Mkn 59 with measured [O III] $\lambda 4363$ are given in Table IV.5 along with the adopted ionization correction factors (ICF) while the heavy element abundances in the two brightest knots of Mkn 71 are given in Izotov et al. (1997). 

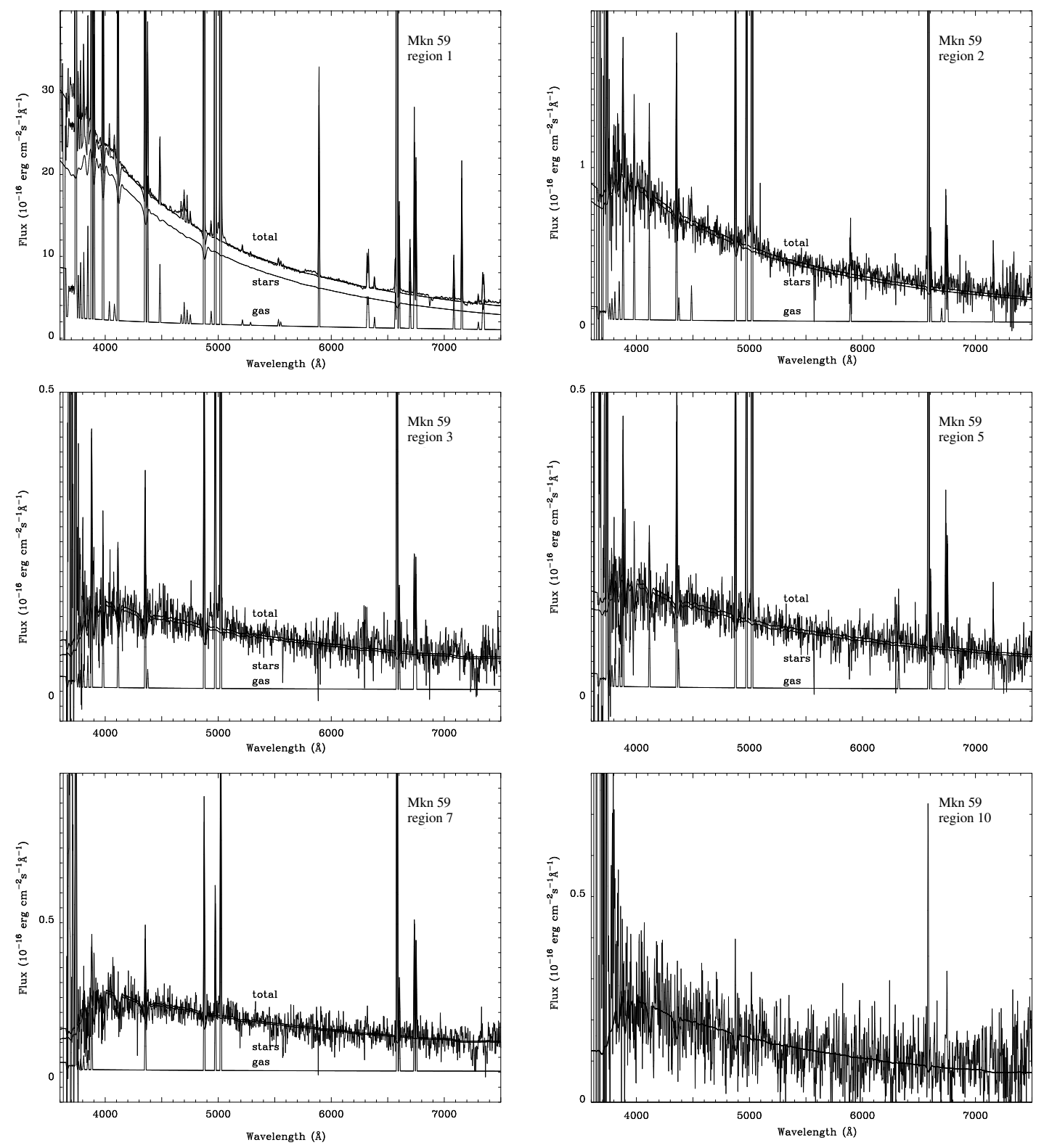

Fig. IV.7. Spectra of different regions of Mkn 59 extracted along the major axis slit at P.A. $=15^{\circ}$ (thin lines). Overplotted are spectral energy distributions (SEDs) of model composite stellar populations and gaseous SEDs. The SEDs of regions No. 3, 7 and 10 are redder than those of the other regions and refer to the underlying stellar component. It is obvious that the contribution of ionized gas to the observed SED is much larger in region 1 than in other regions further to the northeastern direction of the main body of the BCD. 

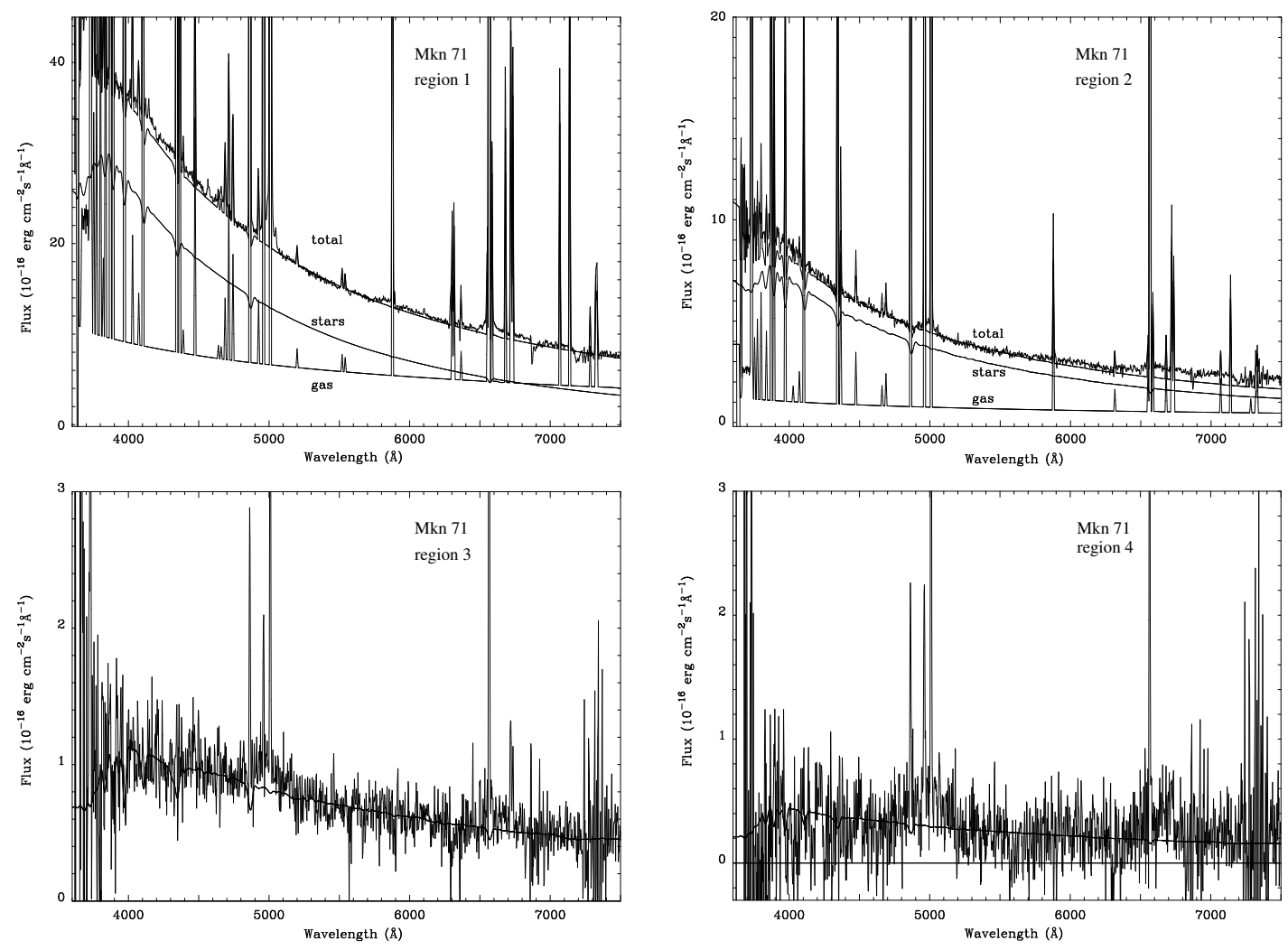

Fig. IV.8. Spectra of the regions in Mkn 71, extracted from the slit at P.A. $=77^{\circ}$ (thin lines). Overplotted are spectral energy distributions (SEDs) of model composite stellar populations and gaseous SEDs. The SED of the region No. 4 is redder than those of other regions and matches the underlying stellar component. Note the large contribution of the ionized gas in region 1.

\section{IV.3 Results}

\section{IV.3.1 Structural properties of the host galaxies}

Recent studies have established that the underlying host galaxy of $\mathrm{iE} / \mathrm{nE}$ BCDs does systematically differ with respect to its central surface brightness $\mu_{E, 0}$ and exponential scale length $\alpha$ from other classes of dwarf galaxies such as dIs and dEs (P96b, Patterson \& Thuan 1996, Marlowe et al. 1997, Papaderos 1998, Salzer \& Norton 1998, Marlowe et al. 1999). This structural dichotomy is evident from Figure IV.9 (adopted from Papaderos 1998) showing that the central surface brightness and exponential scale length of the LSB component of a BCD with an absolute $B$ magnitude $M_{E} \sim-16$ mag are respectively $\gtrsim 1.5$ mag brighter and a factor of $\sim 2$ smaller than in a typical $\mathrm{dI} / \mathrm{dE}$ of equal luminosity. Although there is no sharp limit, a gap around $\mu_{E, 0} \approx 22 B \mathrm{mag} / \square^{\prime \prime}$ separates the host galaxies of $\mathrm{iE} / \mathrm{nE} \mathrm{BCD}$ from other classes of dwarf galaxies. The same diagram shows that more luminous BCDs (i.e. systems with a host galaxy brighter than -16 mag in the $B$ band) follow the same trend populating systematically different areas in the $\mu_{E, 0}-M_{E}$ and $\log \left(\alpha_{E}\right)-M_{E}$-planes than dIs and dEs. In Figure IV.9 

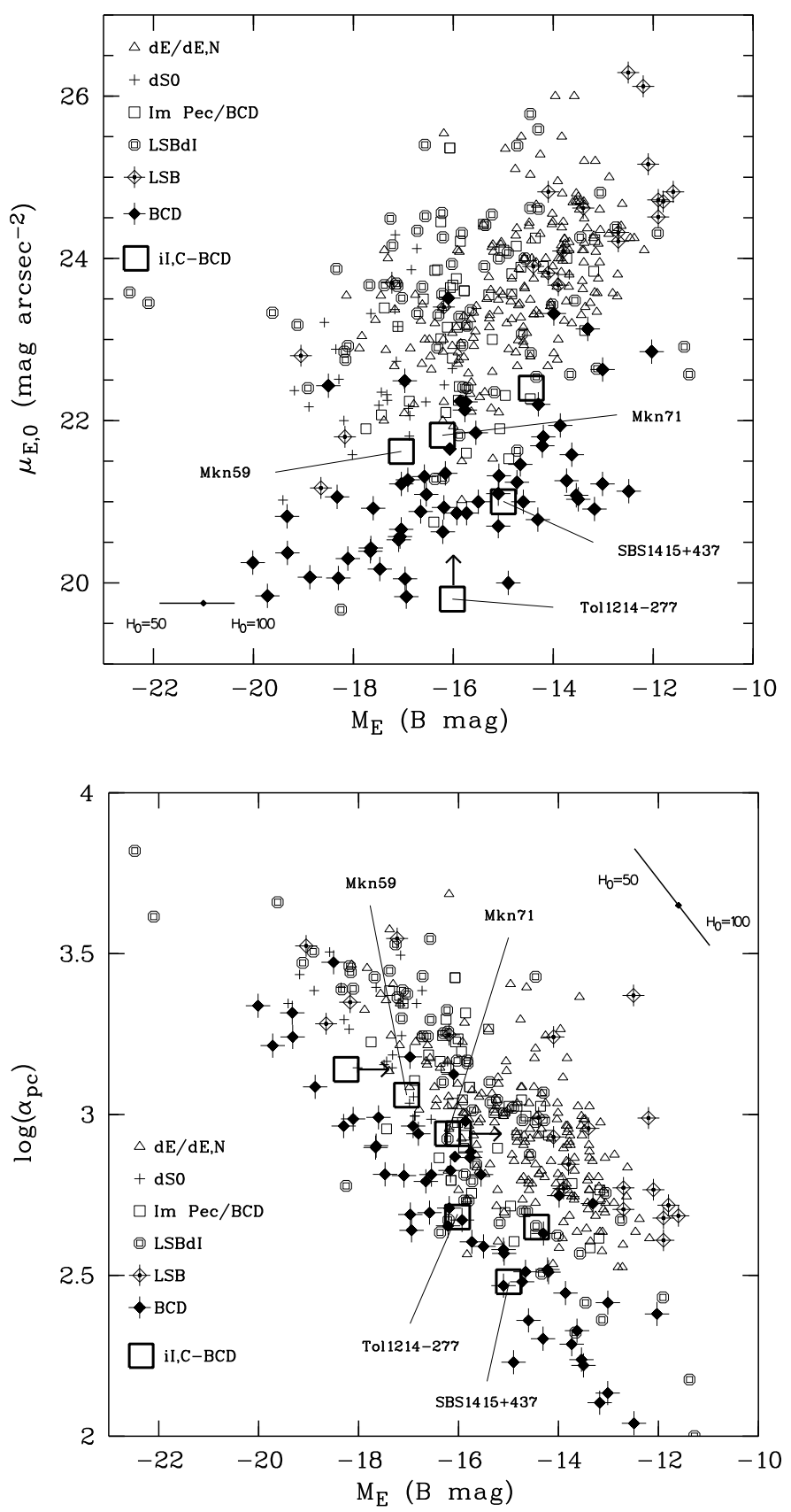

Fig. IV.9. $B$ band structural properties of the underlying exponential components for different types of dwarf and low surface brightness (LSB) galaxies, compiled in Papaderos (1998). The positions of the cometary BCDs Mkn 59, Mkn 71, Mkn 1328, UM 133, UM 417, SBS 1415+437 (Thuan et al. 1999) and Tol 1214-277 (Fricke et al. 2000; cf. Section IV.3.1, this work) are indicated by big squares. top Extrapolated central $B$ surface brightness $\mu_{E, 0}$ vs. absolute $B$ magnitude $M_{E}$ of the LSB components. bottom Exponential scale length $\alpha$ vs. $M_{E}$. Boxes with arrows to the right mark UM 133 (Telles \& Terlevich 1997) and Mkn 1328 (James 1994) and give upper limits for $M_{E}$. Data points for $\mathrm{iE} / \mathrm{nE}$ BCDs and other classes of dwarf galaxies are taken from Drinkwater \& Hardy (1991), P96a, Papaderos (1998), Marlowe et al. (1997), Noeske (1999), Noeske et al. (1999a) and Binggeli \& Cameron (1991, 1993), Caldwell \& Bothun (1987), Bothun et al. (1991), Patterson \& Thuan (1996), Vigroux et al. (1986), Hopp \& Schulte-Ladbeck (1991) and Carignan \& Beaulieu (1989), respectively. 
(left panel) we show with open squares the positions of the LSB components of the iI,C BCDs studied here (values derived in the $B$ band) along with the ones of the iI,C BCDs SBS 1415+437 (Thuan et al. 1999), Tol 1214-277 (Fricke et al. 2000) and UM 417 (Cairós et al. 2000). In the right panel of the same figure we include two further iI,C BCDs for which measurements of the exponential scale length of their LSB components are available, UM 133 (Telles \& Terlevich 1997) and Mkn 1328 (James 1994). It may be seen that the $\mathrm{iI}, \mathrm{C}$ BCDs fit into the gap between typical $\mathrm{iE} / \mathrm{nE}$ BCDs and dIs/dEs except for SBS 1415+437 and Tol 1214-277 which are indistinguishable from typical BCDs in the $\mu_{E, 0}-M_{E}$ and $\log \left(\alpha_{E}\right)-M_{E}$ parameter space.

The uncertain distance to Mkn 59 (cf. Section IV.1) does not affect the results stated above. As can be seen in both panels of Figure IV.9, the vector illustrating a shift to the data points due to a change of the Hubble constant is approximately parallel to the sequences of data points for $\mathrm{iE} / \mathrm{nE}$ BCDs and other types of dwarf galaxies. For a different distance, Mkn 59 therefore remains within the gap between compact BCDs and dIs/dEs.

\section{IV.3.2 Heavy element and line intensity distributions}

In Figure IV.10 we show the spatial intensity distributions of the $\mathrm{H} \alpha$ and $\mathrm{H} \beta$ lines along with that of the continuum adjacent to $\mathrm{H} \beta$, as determined from the one-dimensional spectra extracted along the slit at P.A. $=59^{\circ}$ of $\mathrm{Mkn} 59$ (cf. Figure 6). It is obvious that all three distributions are spatially coincident. This is also the case for other nebular emission lines, including $\mathrm{He}$ I, [N II], [O II], [O III] and [Ne III], which show the same spatial distribution as the $\mathrm{H} \alpha$ and $\mathrm{H} \beta$ emission lines.

Wolf-Rayet stars were detected in region No. 1 of both, Mkn 59 and Mkn 71. For Mkn 71, 5 late nitrogen WR stars (WN stars) and 2 early carbon WR stars (WC stars) stars were found. The number of WC stars in Mkn 59 was determined to be 13 and that of WN stars to be 40 (Guseva et al. 2000, values adapted to the distances used here). The spatial flux distributions of the blue $\lambda 4650$ and red $\lambda 5808 \mathrm{WR}$ bumps are shown in the right panel of Figure IV.10 along with the one of the nebular He II $\lambda 4686$ emission line. The red bump results primarily from WC stars, the blue bump from WN stars. We remark that the maxima of the $\mathrm{H} \beta$ and WR emission are shifted by 1 pixel $\left(0\right.$.' 69) along the slit at P.A. $=59^{\circ}$ suggesting that the locations of $\mathrm{O}$ and WR stars may slightly differ.

In all one-dimensional spectra of Mkn 59 at P.A. $=59^{\circ}$ the $[\mathrm{O}$ III $] \lambda 4363$ emission line is detected which allows a reliable determination of the electron temperatures and element abundances. Despite the large spatial variations of the oxygen line intensities and their ratios, the oxygen abundance is practically constant over the starburst region within the errors (Figure IV.11). The abundances of other heavy elements (neon, nitrogen, argon, sulphur, iron and chlorine) show the same, nearly constant spatial distribution along the slit with P.A. $=59^{\circ}$ through the center of the giant $\mathrm{H}$ II region. In spite of the small abundance variations within the central part, in most cases $\leq 0.2$ dex, we remark that a weak gradient is present for the abundances of all heavy elements along 

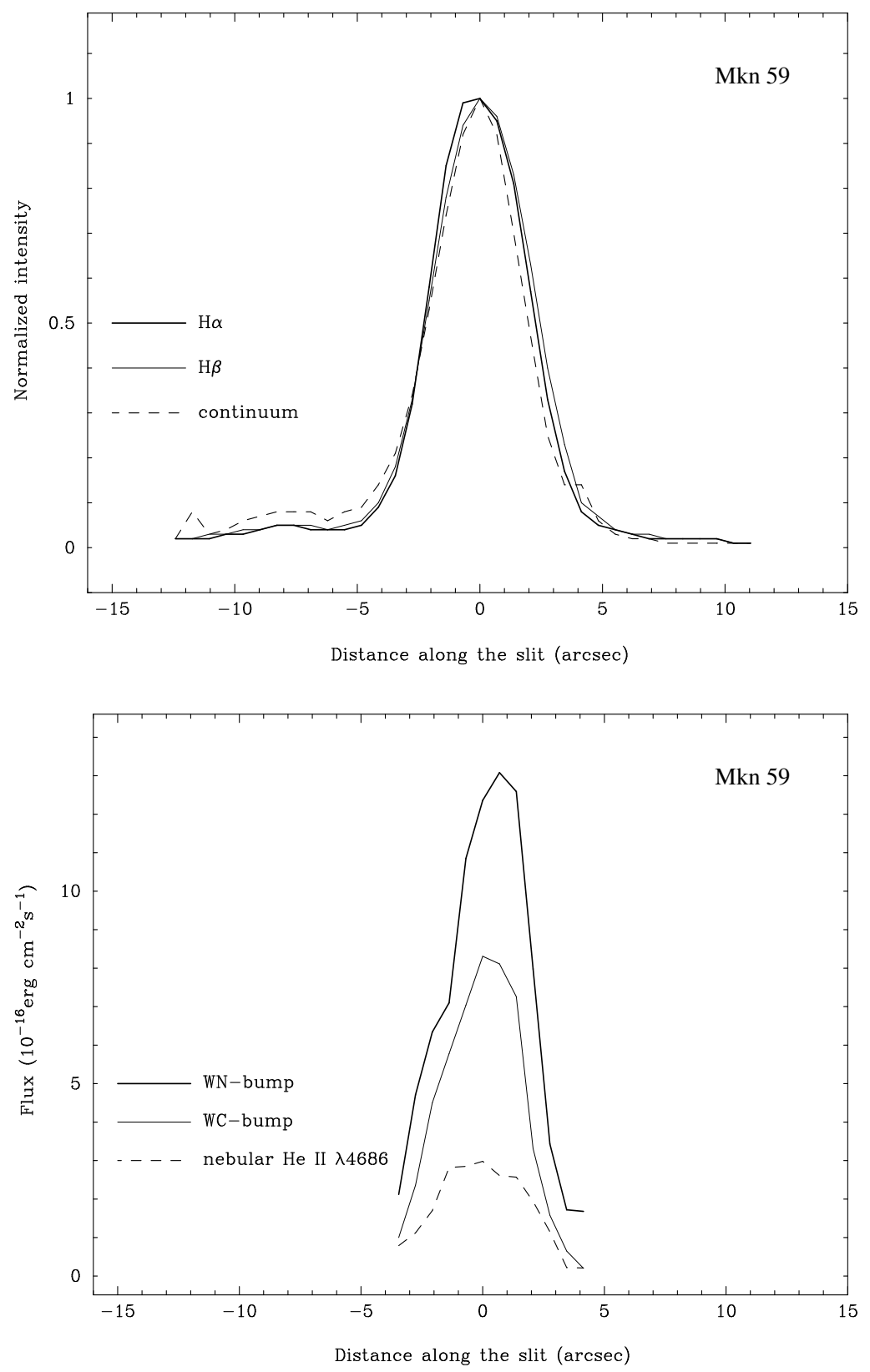

Fig. IV.10. top Spatial distribution of the continuum flux near $\mathrm{H} \beta$ (dashed line) and of the fluxes of the $\mathrm{H} \beta$ and $\mathrm{H} \alpha$ emission lines (thin and thick solid lines, respectively) in Mkn 59 in the direction of P.A. $=59^{\circ}$. The fluxes are normalized to the values at the brightest pixel. bottom Spatial distribution of the WR broad bump fluxes at $\lambda 4650$ (WN bump, thick line), $\lambda 5808$ (WC bump, thin line) and the nebular He II $\lambda 4686$ emission line flux (dashed line) in Mkn 59 along the P.A. $=59^{\circ}$ direction. Note that the maximum of the $\mathrm{WN}$ bump appears to be slightly shifted relative to the maximum of the $\mathrm{H} \beta$ emission line, continuum and nebular flux distribution. 

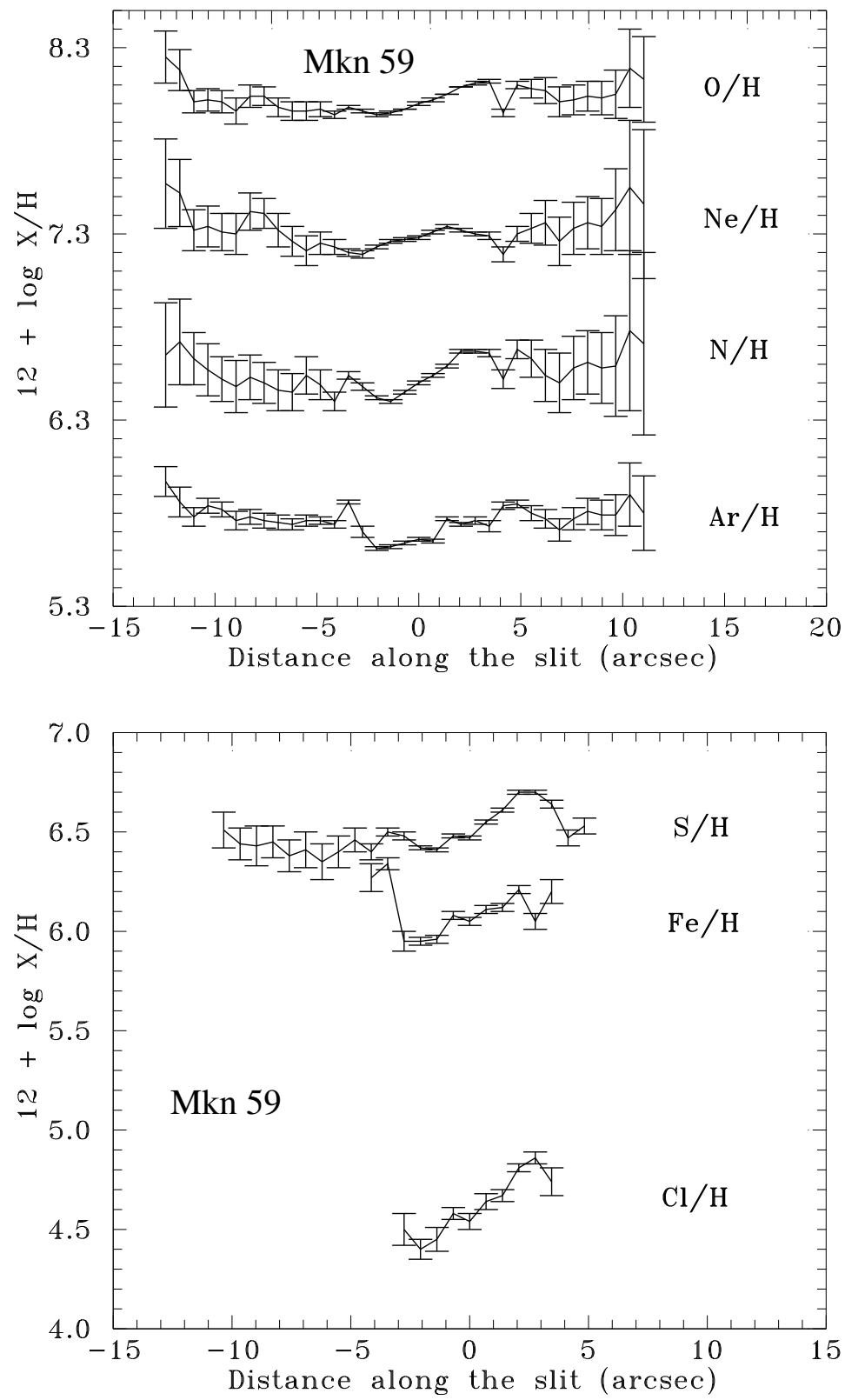

Fig. IV.11. Spatial distributions of the heavy element abundances in Mkn 59 along the slit at P.A. $=59^{\circ}$.

the slit from $-2^{\prime \prime}$ to $+3^{\prime \prime}$, i.e. on scales of $\sim 260 \mathrm{pc}$. This suggests a local heavy element enrichment and may be related to the possible displacement of WR stars relative to $\mathrm{O}$ stars (see above).

In Figure IV.12 we show the spatial distribution of the oxygen abundance in Mkn 59 along the major axis with P.A. $=15^{\circ}$. Three regions where the electron temperature was derived from the [O III] $\lambda 4363 /(\lambda 4959+\lambda 5007)$ flux ratio are indicated by stars, while open circles mark the regions where the [O III] $\lambda 4363$ line was not detected, thus oxygen abundances were derived following van Zee et al. (1998a). The latter abundances 
appear systematically higher compared to those where the electron temperature could be directly constrained utilizing the [O III] $\lambda 4363$ line. However, the differences are still comparable to the intrinsic uncertainty of the order of \pm 0.2 in $\log (\mathrm{O} / \mathrm{H})$ ascribed by van Zee et al. (1998a) to their empirical calibration. Taking this fact into account, as well as the weakness of the [O III] $\lambda 4363$ line in the outer parts of the galaxy, we shall consider the oxygen abundance as constant over the main body of Mkn 59 with an average value of $12+\log (\mathrm{O} / \mathrm{H})=8.0$.

The same analysis for Mkn 71 yields for the two brightest regions (regions 1 and 2; Figure IV.1, right), in which the [O III] $\lambda 4363$ line is observed, heavy element abundances of $12+\log (\mathrm{O} / \mathrm{H})=7.79$ and 7.77 , respectively. The oxygen abundance in the regions 3 and 4 derived from the empirical calibration method described in van Zee et al. (1998a) is larger by $\sim 0.3$ dex.

\section{IV.3.3 Population synthesis models}

For an analysis of the stellar content of the young ionizing clusters, we used the spectral energy distributions (SEDs) calculated by Schaerer \& Vacca (1998) for heavy element mass fractions and ages in the range between $Z=0.001-0.02$ and $t=0.1-10 \mathrm{Myr}$, respectively. For ages $t \geq 10 \mathrm{Myr}$, we calculated a grid of SEDs for stellar populations with ages ranging from $10 \mathrm{Myr}$ to $10 \mathrm{Gyr}$ in time steps of $\Delta \log t[\mathrm{yr}]=0.1$ and a heavy element mass fraction of $Z=0.002$, using isochrones from Bertelli et al. (1994) and the compilation of stellar atmosphere models from Lejeune et al. (1998). An initial mass function (IMF) with a Salpeter slope (2.35) and lower and upper mass limits of $0.6 M_{\odot}$ and $120 M_{\odot}$ was adopted.

To study the age of the stellar populations in the galaxies and to compare the results of the spectral analysis with broad band photometric data, it is necessary to take into account both the stellar and ionized gaseous emission in the spectra. For this purpose, the stellar SED has to be separated from the gaseous emission following the procedure described by Guseva et al. (1998) and Papaderos et al. (1998). We added stellar SEDs calculated for instantaneous bursts with different ages ("single stellar populations"; SSPS) to the observed gaseous emission SED to match the total SED. The contribution of the gaseous emission was scaled to the stellar emission by the ratio of the observed equivalent width of the $\mathrm{H} \beta$ emission line to the equivalent width of $\mathrm{H} \beta$ expected for pure gaseous emission. To calculate the gaseous continuum SED at each region along the slit, the observed $\mathrm{H} \beta$ flux and the electron temperatures were derived from the respective spectrum. The contributions of bound-free, free-free, and two-photon processes to the continuum emission were then calculated for the spectral range from 0 to $5 \mu \mathrm{m}$ (Ferland 1980, Aller 1984).

\section{IV.3.4 The ages of the stellar populations}

The number of $\mathrm{O}$ stars within the giant $\mathrm{H}$ II region complexes at the southeastern tips of Mkn 59 and Mkn 71 was derived from the $\mathrm{H} \beta$ flux to $\approx 4740$ and 2010, respectively, following the prescriptions by Guseva et al. (2000). As stated in Section IV.3.2, WR stars were detected in both BCDs, which, together with the large number of $\mathrm{O}$ stars, 
contribute strongly to the total SED. Taking into account the average metallicities, the ages of the brightest $\mathrm{H}$ II regions in which WR stars were detected cannot exceed 4 to 5 Myr (e.g. Schaerer \& Vacca 1998).

In Figs. IV.7 and IV.8 the modelled stellar SEDs, the gaseous SEDs and their coadded spectral distributions (marked as "total") are shown superposed on the observed spectra for different regions of the BCDs. For nearly all spectra, a good agreement is achieved between the observed and modelled SEDs. Only in the case of region 2 of Mkn 71 (see Figure IV.1, right and Figure IV.8), the agreement is not as good due probably to an imperfectly focussed telescope.

Assuming that the observed gas emission is not caused by local SF, but due to photoionization or shock ionization by SF outside the observed region, the observed SEDs are in some cases reproducible with one SSP of intermediate age (few $10^{8} \mathrm{yr}$ ) only; mostly, however, reproducing the observed SEDs required a superposition of SSPs with different ages and mass fractions. Tables IV.6 and IV.7 list the ages and relative mass fractions of the old and young SSPs which provide the best fits to the observed SEDs at different slit positions. The models imply respective ages of 4 and 2.9 Myr for the stellar population formed in the bright $\mathrm{H}$ II region complexes in Mkn 59 and Mkn 71 (slit regions 1) while for other slit positions the age of the younger stellar continuum was inferred to few 10 Myr. The ages of the old SSPs could be constrained to $\lesssim 2$ Gyr. Within the uncertainties of the methods applied, an upper limit of $\sim 3-$ $4 \mathrm{Gyr}$ is possible (see the following subsections), whereas even higher ages appear untenable. One has, however, to keep in mind that present spectrophotometric dating methods cannot definitely rule out the presence of a small fraction of even older stars, which due to their high $M / L$ would barely contribute to the SED.

\section{IV.3.4.1 Uncertainties of the population synthesis models}

The goodness of the spectral fits was found to sensitively depend on the mass fraction and ages of the adopted young and old SSPs. For instance, for region 1 of Mkn 59, increasing the age of the young SSP from 4 to $5 \mathrm{Myr}$ yielded a satisfactory fit, but a $6 \mathrm{Myr}$ old SSP resulted in systematic residuals between the observed and modelled SED. The age of the old SSPs could be constrained with an accuracy of $\approx 1 \mathrm{Gyr}$ at slit regions where young stellar sources provide a minor contribution to the light (regions 3, 7 and 10 in Mkn 59 and region 3 in Mkn 71). In e.g. region 7 of Mkn 59, old SSPs up to 2 Gyr could still reproduce the observed SED, while already a $3 \mathrm{Gyr}$ old SSP produced obvious residuals to the observed spectrum. A comparison of the observed and modelled $E W(\mathrm{H} \beta)$ yielded further constraints to resolve ambiguities between different SED solutions. The assumption of high ages for the old SSP requires an increased contribution by the young SSP to reproduce the blue continuum, which in turn results in too high $E W(\mathrm{H} \beta)$ as compared to the observed value.

Given that the metallicities of the stellar populations in BCDs may be lower than those of the ionized gas (Calzetti 1997, Guseva et al. 1998, Mas-Hesse \& Kunth 1999), we computed a set of models varying the metallicity of the SSPs around $Z=0.002$ by $0.5 \mathrm{dex}$, the smallest stepsize our model libraries allowed. Models computed on the 
Table IV.6. Synthetic colours of a mixture of young and old populations in Mkn 59

\begin{tabular}{|c|c|c|c|c|c|c|c|c|}
\hline \multicolumn{9}{|c|}{$\overline{\text { region } \log t \text { relative mass } \log t \text { relative mass }(B-V)(B-R)(R-I)(V-K)}$} \\
\hline \multicolumn{9}{|c|}{ a) Stellar and gaseous emission } \\
\hline 1 & 6.6 & 0.7 & 9.0 & 0.3 & 0.17 & -0.01 & -0.32 & 0.12 \\
\hline 2 & 6.9 & 0.6 & 9.3 & 0.4 & 0.03 & 0.09 & -0.03 & 0.64 \\
\hline 3 & 8.7 & 1.0 & $\ldots$ & $\ldots$ & 0.29 & 0.52 & 0.28 & 1.93 \\
\hline 4 & 7.4 & 0.1 & 9.3 & 0.9 & 0.21 & 0.42 & 0.25 & 1.59 \\
\hline 5 & 7.4 & 0.1 & 9.3 & 0.8 & 0.28 & 0.44 & 0.16 & 1.46 \\
\hline 6 & 7.0 & 0.3 & 9.3 & 0.7 & 0.04 & 0.21 & 0.24 & 1.39 \\
\hline 7 & 8.7 & 1.0 & $\ldots$ & $\ldots$ & 0.28 & 0.58 & 0.30 & 2.03 \\
\hline 8 & 7.4 & 1.0 & $\ldots$ & $\ldots$ & 0.05 & 0.24 & 0.27 & 1.54 \\
\hline 9 & 7.0 & 0.1 & 9.3 & 0.9 & 0.11 & 0.30 & 0.28 & 1.51 \\
\hline 10 & 8.3 & 1.0 & $\ldots$ & $\ldots$ & 0.15 & 0.33 & 0.32 & 1.90 \\
\hline \multicolumn{9}{|c|}{ b) Stellar emission } \\
\hline 1 & 6.6 & 0.7 & 9.0 & 0.3 & -0.15 & -0.17 & 0.00 & 0.20 \\
\hline 2 & 6.9 & 0.6 & 9.3 & 0.4 & -0.04 & 0.02 & 0.11 & 0.70 \\
\hline 3 & 8.7 & 1.0 & $\ldots$ & $\ldots$ & 0.28 & 0.54 & 0.39 & 2.08 \\
\hline 4 & 7.4 & 0.1 & 9.3 & 0.9 & 0.18 & 0.43 & 0.33 & 1.70 \\
\hline 5 & 7.2 & 0.2 & 9.3 & 0.8 & -0.01 & 0.11 & 0.19 & 1.22 \\
\hline 7 & 8.7 & 1.0 & $\ldots$ & $\ldots$ & 0.28 & 0.54 & 0.39 & 2.08 \\
\hline
\end{tabular}

Table IV.7. Synthetic colours of a mixture of young and old populations in Mkn 71

\begin{tabular}{|c|c|c|c|c|c|c|c|c|}
\hline \multicolumn{9}{|c|}{$\overline{\text { region } \log t \text { relative mass } \log t \text { relative mass }(B-V)(B-R)(R-I)(V-K)}$} \\
\hline \multicolumn{9}{|c|}{ a) Stellar and gaseous emission } \\
\hline 1 & 6.46 & 1.0 & $\ldots$ & ... & 0.26 & 0.04 & -0.58 & 0.07 \\
\hline 2 & 6.6 & 0.15 & 9.3 & 0.85 & 0.03 & 0.13 & -0.14 & 0.62 \\
\hline 3 & 6.6 & 0.02 & 9.0 & 0.98 & 0.30 & 0.57 & 0.40 & 2.09 \\
\hline 4 & 8.7 & 1.0 & ... & $\ldots$ & 0.28 & 0.54 & 0.39 & 2.08 \\
\hline \multicolumn{9}{|c|}{ b) Stellar emission } \\
\hline 1 & 6.4 & 1.0 & $\ldots$ & ... & -0.19 & -0.25 & -0.08 & -0.42 \\
\hline 2 & 6.6 & 0.15 & 9.3 & 0.85 & -0.07 & -0.03 & 0.09 & 0.67 \\
\hline
\end{tabular}

assumption of a metallicity other than $Z=0.002$ failed to adequately reproduce the observed spectrum, suggesting that the metallicity of the ionized gas is similar to that of the stellar population.

Alternatively, to constrain the age of the underlying galaxies, not only an instantaneous burst was considered, but also an extended episode of moderate SF at a constant rate. This way, the presumably complex formation histories of the galaxies were bracketed between two limiting cases.

In the same way as described in Section IV.3.3, a grid of model SEDs was calculated for stellar populations that were formed in extended SF episodes which started and ended at different times. The best-fitting solutions for e.g. Mkn 71 correspond to a 
formation episode of the underlying galaxy which started $\sim 2$ Gyr ago and ended $\sim 10 \mathrm{Myr}$ ago. Within the uncertainties, a SF episode that began up to $3 \mathrm{Gyr}$ ago is possible; higher ages of the underlying galaxies would require a very recent $(<10 \mathrm{Myr}$ ago) termination of their formation episode to reproduce the observed SEDs. Such a recently ongoing SF activity in the underlying galaxy in Mkn 71 can be rejected from the colour-magnitude diagrams (Figure IV.4); in the left panel (a), obtained at the starburst region NGC 2363, stars are absent between the actual burst (younger than $\sim 10 \mathrm{Myr}$ ) and the last SF episode of the underlying galaxy which seems to have occurred more than $\sim 20 \mathrm{Myr}$ ago (note the red supergiants at the tip of the $25 \mathrm{Myr}$ isochrone). Also in the right panel (b), no stars much younger than $\sim 20 \mathrm{Myr}$ are present. This indicates that, in the galaxy underlying the starburst, no significant SF occured during the last $\gtrsim 20 \mathrm{Myr}$, in agreement with the ground-based CMDs by Aparicio et al. (1995). A similar analysis for Mkn 59 indicates, within the unvertainties, an upper age limit of $\sim 4 \mathrm{Gyr}$.

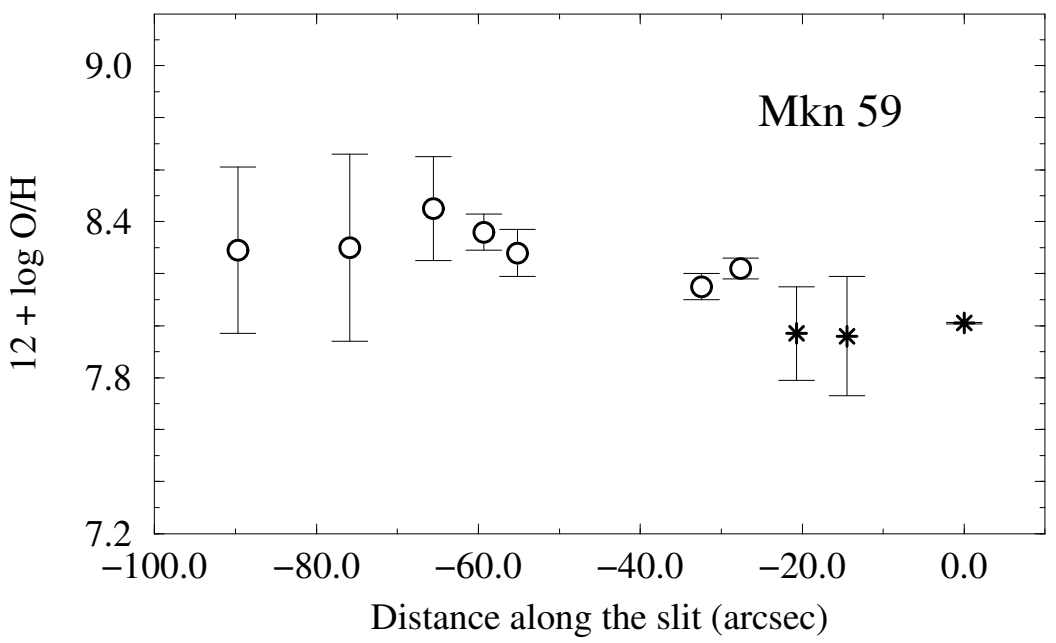

Fig. IV.12. Spatial distribution of the heavy element abundances in Mkn 59 along the slit at P.A. $=15^{\circ}$. Stars mark the regions where the $[\mathrm{O}$ III $] \lambda 4363$ emission line was detected, open circles correspond to regions where the oxygen abundance was derived from the empirical relation by van Zee et al. (1998a).

\section{IV.3.4.2 Consistency with imaging data; further implications}

The $E W(\mathrm{H} \beta)$ of region 1 in Mkn 59 and Mkn 71 amount to $150 \AA$ (Table IV.3) and $316 \AA$ (Izotov et al. 1997), respectively. For these regions the broad band colours calculated from the synthetic SEDs, including and ignoring gaseous emission (Tables IV.6 and IV.7) differ from each other by up to $0.5 \mathrm{mag}$. This demonstrates that a reliable dating of stellar populations within a starburst environment requires a correction for the colour shift induced by ample gaseous emission. 
The consistency of the observed broad band colours with the spectral population synthesis results was checked by extracting the areas covered by the spectrograph slits from the colour maps of Mkn 59 and Mkn 71 (cf. Section IV.2.1.1). Figure IV.13 shows the modelled and observed colours along the slits after the latter were transformed to the Johnson-Cousins system (Bessell 1990). Generally, the observed and synthesized
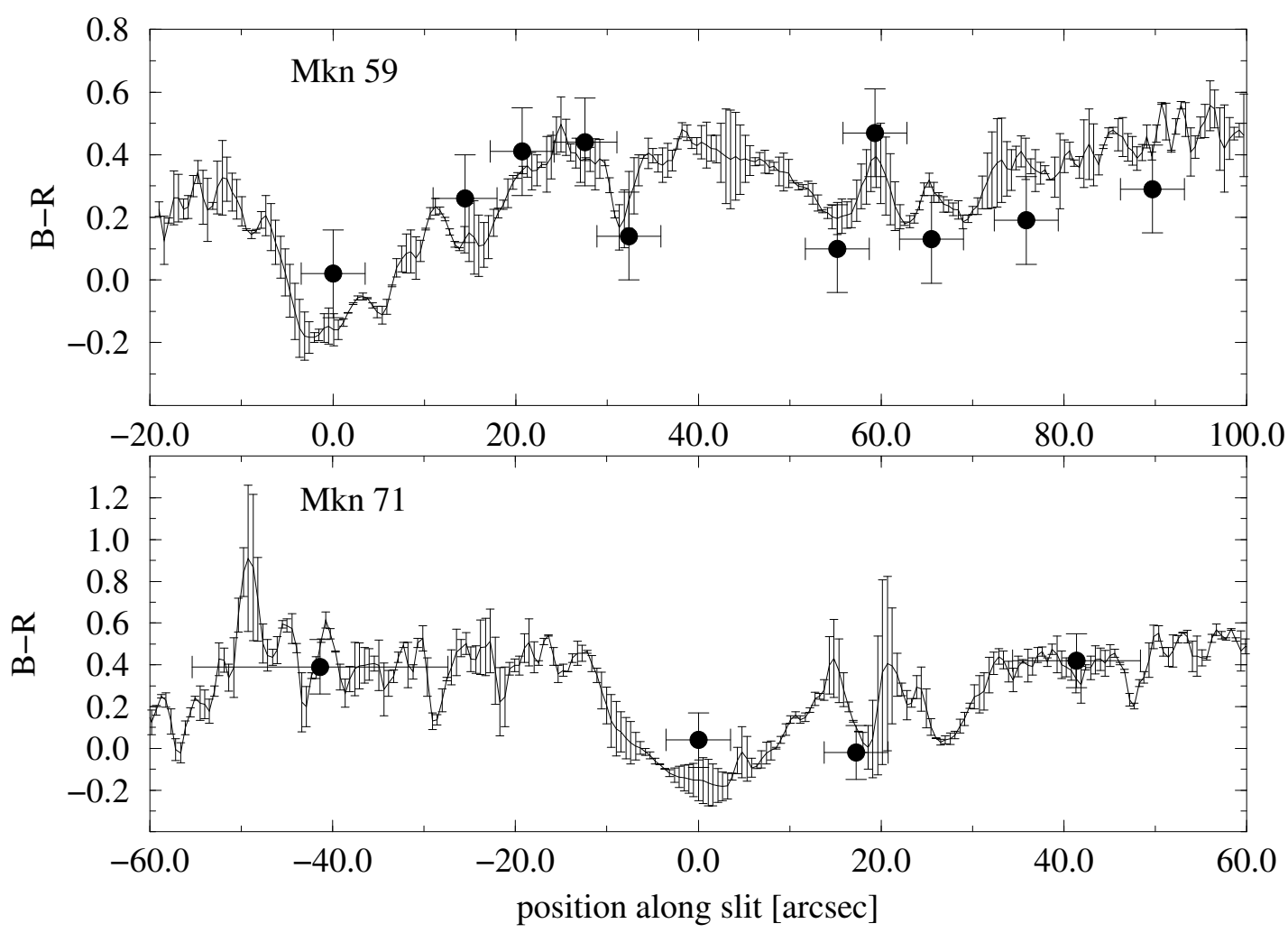

Fig. IV.13. Observed colour distributions along the slits extracted from colour maps, compared to the colours computed from population synthesis solutions (black dots) for the analyzed subspectra. All colours are derived on the assumption of a uniform extinction and are transformed to the standard Johnson-Cousins UBVRI system specified by Bessell (1990), Bessell et al. (1998). top: $\operatorname{Mkn} 59$, P.A. $=15^{\circ}$, bottom: Mkn 71, P.A. $=77^{\circ}$. Vertical error bars of the synthetic colours include cumulative calibration uncertainties of the colour maps and population synthesis models, and of the transformation between the filter systems; horizontal error bars give the length of regions along the slit from which the individual subspectra were extracted. The alignment uncertainty between the observed and synthetic colors in direction of the slit (i.e. along the abscissa) is $\approx 5^{\prime \prime}$. The error bars of the observed colour distributions give the $1 \sigma$ colour change for a $2^{\prime \prime}$ shift of the extracted colour map region perpendicular to the slit direction.

colours agree well with each other (within $1 \sigma$ uncertainties), given that i) the synthesized colours are averaged values over regions covering several arcsec length along the slit, and ii) a minor displacement between the positions of a region in the colour map and of the slit may significantly alter the observed colours (see Figure IV.6 and the 
$1 \sigma$-variations of the observed colours in Figure IV.13), as the H II regions are typically very compact.

Transforming the colours of the LSB component as derived from the colour profiles (Figure IV.3) to the Johnson-Cousins system yields a $B-R \lesssim 0.6$ for Mkn 59 and Mkn 71. This is in good agreement with the upper limits of the colours derived from the spectra (cf. Tables IV.6 and IV.7). The constancy of the colour profiles for photometric radii where the starburst light contribution becomes negligible (i.e. for $R^{*} \gtrsim P_{25}$ ) indicates that in the underlying galaxies population gradients are absent. Therefore, the upper age limits as being derived for either BCD along the spectrograph slits can be considered to be valid for the entire underlying galaxy.

\section{IV.4 Discussion}

\section{IV.4.1 The metallicity distribution}

Compared to BCDs in general, which show a metallicity distribution that peaks around $\approx 1 / 10 Z_{\odot}$ and steeply decays towards lower metallicities (Kunth \& Sargent 1986), the metal abundances of Mkn $59\left(1 / 8 Z_{\odot}\right)$ and Mkn $71\left(1 / 14 Z_{\odot}\right)$ are not exceptional. The nearly constant metallicity along the major and minor axes of Mkn 59, as well as along the minor axis of Mkn 71 (Figs. IV.11 and IV.12; see also González-Delgado et al. 1994), suggests that large-scale mixing processes in the ISM of both BCDs were at work on time scales of a few $10^{6} \mathrm{yr}$. Otherwise, one would expect measurable metallicity enhancements in the vicinity of regions of ongoing or recent SF. Furthermore, the transport of metal-enriched warm and hot gas on scales of up to $\sim 1 \mathrm{kpc}$ from the starburst region, an observational signature of which is the formation of supergiant shells, is commonly seen in the ISM of star-forming dwarf galaxies (cf. e.g. Marlowe et al. 1995, Hunter \& Gallagher 1997, Bomans et al. 1997, Brinks \& Walter 1998, Papaderos \& Fricke 1998, Strickland \& Stevens 1999; cf. also Figure IV.2, this work). The dilution of heavy elements in the vicinity of starburst regions may even be powered by galactic outflows. These were predicted by numerical models to develop within dwarf galaxies (Vader 1986, De Young \& Gallagher 1990, De Young \& Heckman 1994, Mac Low \& Ferrara 1999) and proved necessary to account for the much lower metallicities observed in BCDs with respect to those predicted from closed-box evolutionary synthesis models (Krüger 1992, Lisenfeld \& Ferrara 1998). The development of such a large-scale perturbation of the ambient gaseous component does not appear unreasonable given the high-velocity $\left(\sim 10^{3} \mathrm{~km} \mathrm{sec}^{-1}\right)$ gaseous motions revealed spectroscopically by Izotov et al. (1996) and Roy et al. (1991, see also Figure IV.2, this work) in Mkn 59 and Mkn 71, respectively.

The small variations of the heavy element abundances along the giant $\mathrm{H}$ II region complex of Mkn 59 (Section IV.3.2), as well as the possible spatial offset of the O-and WR stars, may be explained by a relocation of SF processes within a time span of few Myr. Propagating SF on a linear scale of $\sim 400 \mathrm{pc}$ within the last $10 \mathrm{Myr}$ has also been suggested by Drissen et al. (1999), to account for the age differences of the young star clusters NGC 2363 I and II within Mkn 71. 


\section{IV.4.2 Morphology vs. structural properties and age}

The two iI,C BCDs investigated here appear similar with respect to the age and structure of their exponentially distributed host galaxies. This raises the question of whether the entire class of iI,C BCDs shares, besides a morphological resemblance, a set of common physical properties, such as an intermediate age and structural properties bridging the gap between $\mathrm{iE} / \mathrm{nE}$ BCDs and dIs/dEs. A literature search for data on other nearby cometary BCDs does indeed provide some support to this hypothesis.

Mkn 1328/VCC 1374: NIR surface photometry (James 1994) yields an exponential scale length of $\alpha=0.88 \mathrm{kpc}$ for its LSB component. With its integrated absolute $B$ magnitude of $-16 \mathrm{mag}$, and taking into account that a burst raises the $B$ luminosity of a BCD by typically 0.75 mag (P96b, Salzer \& Norton 1998), Mkn 1328 resides presumably in between the parameter spaces populated by BCDs and dIs in the $\log \left(\alpha_{E}\right)-$ $M_{E}$-plane (cf. Figure 9). From the integral $(B-H)$ colour of $\approx 0.6 \mathrm{mag}$, the age of its host galaxy may be estimated to $\lesssim 6$ Gyr, following the predictions by Krüger (1992) and Krüger et al. (1995) when a burst parameter $b<0.01$ is adopted. The metallicity was determined to $1 / 8 Z_{\odot}$ (Kinman \& Davidson 1981).

UM 133: For the observed metallicity $\left(1 / 17 Z_{\odot}\right.$, Telles 1995$)$, the integral $(R-I)$ colour of 0.4 mag derived by Telles \& Terlevich (1997) is consistent with an age of $\sim 4$ Gyr. With $M_{V}=-18.25$, and an exponential scale length of $\alpha=1.39 \mathrm{kpc}$ for its LSB component (Telles \& Terlevich 1997), this object falls also into the gap between BCDs and dIs.

$U M 417\left(Z \approx 1 / 13 Z_{\odot}\right.$; Campos-Aguilar at al. 1993). Surface photometry of the LSB component yields an intermediately compact structure, too $\left(\alpha_{E}=0.45 \mathrm{kpc}\right.$, $M_{E}=-14.4 B$ mag and $\mu_{E, 0}=22.4 B \mathrm{mag} / \square^{\prime \prime}$; Cairós et al. 2000). The same authors deduce a $(B-V)$ color for the LSB component of $\lesssim 0.5 \mathrm{mag}$, compatible with an age of few Gyr.

SBS 1415+437 (Thuan, Izotov \& Foltz 1999): With a probable age of $\lesssim 100 \mathrm{Myr}$ and an extremely low metallicity $\left(1 / 21 Z_{\odot}\right)$, this galaxy is a young galaxy candidate. Its LSB component, however, does not fit within the structural gap between BCDs and dIs; with $\mu_{E, 0}=21.0 \mathrm{~V} \mathrm{mag} / \square^{\prime \prime}, \alpha_{E}=0.30 \mathrm{kpc}$ and $M_{E}=-14.95 \mathrm{~V}$ mag, it is rather comparable to a compact BCD. On the other hand, as this object apparently still undergoes its first major episode of SF, it is likely that its stellar LSB component has not yet been fully built but, as suggested by the age gradient along its major axis, delineates the trail along which SF has occurred.

Tol 1214-277: Similar to SBS 1415+437, this is an extremely low-metallicity iI,C BCD $\left(Z \approx Z_{\odot} / 23\right.$, Terlevich et al. 1991). Recent surface photometry studies with the VLT (Fricke et al. 2000) yield $\alpha_{E} \approx 0.48 \mathrm{kpc}$ and $M_{E} \approx-16 B$ mag. The average colours of the LSB component, $(U-B) \lesssim-0.4$ mag and $(B-R) \approx+0.3 \mathrm{mag}$, suggest an unevolved galaxy with an age of few $10^{8} \mathrm{yr}$.

The examples given above call for a further investigation of the following two hypotheses:

(i) Cometary BCDs are relatively young objects, with ages not exceeding a few Gyr, thus systematically younger than the majority of BCDs classified iE/nE. 
(ii) Except for the candidate young extremely metal-deficient galaxies $\left(\tau \sim 10^{8} \mathrm{yr}\right)$ among them, cometary BCDs show structural properties of their host galaxies being typically intermediate between those of $\mathrm{iE} / \mathrm{nE}$ BCDs and dIs/dEs.

Provided that the above hypotheses will be strengthened by an investigation of a larger sample of nearby iI,C BCDs, one may expect the iI,C morphology to occur more frequently in dwarf objects at higher redshifts. Indeed, examples of high- $z$ galaxies displaying a comet-like morphology have frequently been reported (cf. e.g. Dickinson 1996 for a galaxy cluster at $z=1.15$ ). Furthermore, a few medium-redshift objects with cometary morphology were included in the sample of Gúzman et al. (1998, Figure 1), notably the Faint Blue Galaxy (FBG) HERC 13088, at a redshift $z=0.436$. With a total luminosity of $M_{B}=-21.5 \mathrm{mag}$ and an exponential scale length $\alpha \approx 2.8 \mathrm{kpc}$, this galaxy appears an upscaled version of the nearby iI,C BCDs studied here. It is worth noting that the rest-frame $(B-V)$ colours of the LSB host of the latter FBG, $\sim 0.35$ mag, also suggest an age of $\lesssim 5$ Gyr.

These findings provide support to the hypotheses proposed in Section 1, namely that a strong off-center burst ontop a dwarf LSB component as observed in iI,C BCDs is primarily not a stochastic event but occurs in systems considerably younger than $\mathrm{iE} / \mathrm{nE} \mathrm{BCDs}$, probably differing from the latter with regard to their structural properties. These results emphasize the need for a detailed investigation of the processes leading to the development of iI,C morphology in dwarf galaxies. We shall briefly remark on that issue in the next section.

\section{IV.4.3 An elongated structure of high gas density?}

In the majority of BCDs, the centrally concentrated H II complexes where massive SF takes place are typically found to almost coincide with maxima in the surface density of an extended H I envelope (Taylor et al. 1994, Simpson 1995, van Zee et al. 1998b). Since a high gas density is necessary to sustain SF, such a condition is expected to be fulfilled near the end of the stellar body of an iI,C BCD. Age gradients of the starforming regions along the stellar body's major axis seem to be frequent attributes of such a system (Barth et al. 1994; Aparicio et al. 1995; Thuan et al. 1999). These may be plausibly explained as due to propagation of SF activity (cf. Thuan et al. 1987) along the major axis of an elongated $\mathrm{H}$ I body.

Given these observational results, it seems that the H I halos of iI,C BCDs have elongated central concentrations with major axes that coincide with those of the stellar bodies. Indeed, low-resolution H I maps of Mkn 59 (Wilcots et al. 1996) and Mkn 71 (Wevers et al. 1986) show the stellar components to follow prolate central concentrations within elongated $\mathrm{H}$ I clouds. A similar distribution of the gaseous halo does not seem rare among magellanic irregulars (Wilcots et al. 1996) and has been interferometrically mapped also in BCDs in different evolutionary stages (see e.g. II Zw 40, van Zee et al. 1998b, and IZw 18, van Zee et al. 1998c). Moreover, the young BCD SBS 0335-052 (Izotov et al. 1990) is forming within an elongated gas cloud with a probably primordial chemical composition (Thuan \& Izotov 1997, Lipovetsky et al. 1999). 
It might be argued that stellar bodies and $\mathrm{H}$ I concentrations of iI,C BCDs merely represent edge-on disks, as suggested by the slow rotation found for the $\mathrm{H}$ I envelopes of Mkn 59 and Mkn 71 as well as for the ionized gas of SBS 1415+437 (Thuan et al. 1999). The generally exponential surface brightness profiles of their stellar LSB hosts (cf. Figure IV.3) do not serve as a proof of a disk structure (Freeman 1970), since exponential SBPs are also observed for e.g. small spheroidal systems (Binggeli et al. 1984) and bars in late-type spirals (Elmegreen et al. 1996). However, the assumption of edge-on disks would demand the presence of numerous less-inclined iI,C BCDs, i.e. relatively blue disks with a single starburst in their outskirts, in contradiction to morphological studies of dwarf galaxies.

\section{IV.4.3.1 iI,C systems vs. old iE/nE BCDs}

Considering that only little data is available for BCDs with morphological types other than $\mathrm{iE} / \mathrm{nE}$, the construction of a tentative age-morphology sequence for gas-rich dwarf galaxies appears premature. The evolution of the different types of dwarf galaxies as well as their possible relations among each other are still sketchy, especially the triggering and effects of starbursts as well as the role of the Dark Matter (see Thuan 1985, Davies \& Phillipps 1988, P96b, Meurer 1998, Swaters 1998, Marlowe et al. 1997, 1999; van Zee et al. 1998b, Gil de Paz et al. 1999).

However, we argue that their relatively low ages would make iI,C BCDs a possible link between the extremely young $(\tau \sim 100 \mathrm{Myr})$ dwarf galaxy candidates, most of which belong to the $\mathrm{i} 0$ class, and the evolved $\mathrm{iE} / \mathrm{nE} \mathrm{BCDs}$, for which a lower age limit of 2 Gyr has been invariably derived (Krüger \& Fritze-v.Alvensleben 1994, Noeske 1999). In the young BCDs investigated thus far, a propagation of the SF activity has been observed (Papaderos et al. 1998, Izotov et al. 1999, Thuan et al. 1999). Thereby, it is conceivable that after a period of several $10^{8} \mathrm{yr}$, these objects will gradually develop a cometary morphology with the youngest and most active star-forming regions located at one end of an elongated stellar body.

\section{IV.5 Summary and conclusions}

Aiming at a better understanding of the different morphological subclasses of Blue Compact Dwarf Galaxies (BCDs) in the context of the evolution of star-forming dwarf galaxies, we have started a study of the "cometary" (iI,C ) subclass of BCDs. Contrary to the majority of BCDs where SF is confined to the inner part of an old circular or elliptical stellar LSB host, iI,C BCDs exhibit intense starburst activity close to one end of an elongated irregular LSB component. This intriguing morphology prompts two hypotheses: (i) iI,C BCDs are in fact dwarf irregulars (dIs) observed during a major stochastic enhancement of their otherwise moderate SF activity and (ii) a set of physical properties of the gaseous and stellar components favours the ignition of a burst with an amplitude comparable to that typically observed in other BCDs at the outskirts of an older LSB component. 
For the two nearby cometary BCDs Markarian 59 and Markarian 71, a variety of deep ground-based and HST photometric and spectrophotometric data are available. To investigate the structural properties of the LSB component we derived surface brightness profiles (SBPs), corrected for systematic effects which can be important at low $S / N$ levels. Photometric properties of the underlying host galaxy and the superposed starburst component were derived by fitting a simple decomposition scheme to the SBPs; the radial extent of the starburst component as derived from this profile decomposition was verified using $\mathrm{H} \alpha$ - and colour maps. The spatial distributions of heavy element abundances in the starburst regions and over the main stellar body were derived using long-slit spectra. After the superposed emission by ionized gas had been semi-empirically modelled and subtracted from the original spectra, a population synthesis analysis was carried out at several positions along each slit to derive the properties of the underlying young and older stellar continuum. For Mkn 71 we also derived $(B-V)$ colour-magnitude diagrams from HST data. The main findings of our analysis may be summarized as follows:

1. The azimuthally averaged intensity distribution of the underlying LSB host galaxy of both iI,C BCDs can be approximated by an exponential fitting law with a central surface brightness and a linear scale length intermediate between those typically inferred for $\mathrm{iE} / \mathrm{nE}$ BCDs and dIs/dEs.

2. Spectral synthesis modeling of the starburst region and the main body implies the presence of an older population with a most probable age $\lesssim 2$ Gyr for the underlying host galaxies of Mkn 59 and Mkn 71. Ages up to 4 Gyr for Mkn 59 and 3 Gyr for Mkn 71 are tenable within the model uncertainties, but higher values are unlikely.

3. The average oxygen abundances were determined to be $12+\log (\mathrm{O} / \mathrm{H})=8.0(1 / 8$ $\left.Z_{\odot}\right)$ for $\mathrm{Mkn} 59$ and $12+\log (\mathrm{O} / \mathrm{H})=7.8\left(1 / 14 Z_{\odot}\right)$ for $\mathrm{Mkn} 71$, which are typical among BCDs. In addition, the metallicity distribution as derived for various elements in the vicinity of the starburst regions and along the major axis of the LSB-body shows only small scatter $(\sim 0.2$ dex $)$, suggesting that mixing of heavy elements has been efficient.

The similarity of the two objects with respect to the ages and structural properties of their LSB components motivated a search for published data on other cometary BCDs. We found that five objects for which the required data are available share similar properties with Mkn 59 and Mkn 71 with respect to the results (1) - (2). These findings suggest that the specific starburst morphology observed in iI,C BCDs comes along with distinct physical properties of their LSB host galaxies, i.e. is not attributable to stochastic processes only. Hypotheses which we consider worth investigating are:

(i) Cometary BCDs are relatively young objects, with ages not exceeding a few Gyr, thus systematically younger than "classical" BCDs of type $\mathrm{iE} / \mathrm{nE}$. If true, the development of iI,C morphology may represent a late evolutionary stage of an i0 BCD before it gradually assumes $\mathrm{iE} / \mathrm{nE}$-characteristics.

(ii) The underlying host galaxies of iI,C BCDs with an age $\gtrsim 1$ Gyr are moderately compact, in the sense that they show central surface brightnesses and exponential scale lengths intermediate between those typically derived for $\mathrm{iE} / \mathrm{nE}$ BCDs and dIs/dEs.

The strongly extranuclear location of the starburst regions and the signatures of propagating star-forming activity along the main stellar body of an iI,C BCD suggest 
that the surface density distribution of cold H I gas in these systems resembles the optical morphology; published H I maps support this assumption. Spatially resolved interferometric studies will be of major importance for assessing the intrinsic processes regulating SF in iI,C BCDs and exploring possible evolutionary links to "classical" $\mathrm{iE} / \mathrm{nE}$ BCDs.

Acknowledgement. N.G.G. and Y.I.I. thank the Universitäts-Sternwarte of Göttingen for warm hospitality. We acknowledge the support of Volkswagen Foundation Grant No. I/72919. Research by K.G.N, P.P. and K.J.F. has been supported by Deutsche Forschungsgemeinschaft (DFG) grant FR 325/50-1, Deutsche Agentur für Raumfahrtangelegenheiten (DARA) GmbH grants 50 OR 94076 and 50 OR 9907 7. We thank the referee, Dr. R.C. Dohm-Palmer, for helpful comments and suggestions and C. Möller for providing us with a calibration spectrum. P. Papaderos thanks K. Bischoff for his assistance during the observations at Calar Alto. T.X. Thuan thanks the partial financial support of NSF grant AST-9616863.

\section{References}

Aller, L. H. 1984, Physics of Thermal Gaseous Nebulae (Dordrecht: Reidel)

Anders, E., Grevesse, N. 1989, Geochim. Cosmochim. Acta 53, 197

Aparicio, A., Cepa, J., Gallart, C., et al., 1995, AJ 110, 212

Babul, A., Rees, M.J., 1992, MNRAS 255, 346

Barth, C.S., Cepa, J., Vilchez, J.M., and Dottori, H.M., 1994, AJ 108, 2069

Bertelli, G, Bressan, A., Chiosi, C., Fagotto, F., Nasi, E., 1994, A\&AS 106, 275

Bessell, M.S., 1990, PASP 102, 1181

Bessell, M.S., Castelli, F., Plez, B., 1998, A\&A 333, 231

Binggeli, B., Cameron, L.M., 1991, A\&A 252, 27

Binggeli, B., Cameron, L.M., 1993, A\&AS 98, 297

Binggeli, B., Sandage, A., Tarenghi, M., 1984, AJ 89, 64

Bomans, D.J., Chu, Y.-H., Hopp, U., 1997, AJ 113, 1678

Bothun, G.D., Impey, C.D., Malin, D.F., 1991, ApJ 376, 404

Brinks, E., Walter, F., 1998, in "The Magellanic Clouds and Other dwarf Galaxies", Braun, J.M., Richtler, T. (eds.). Bonn: Shaker Verlag, p.1

Brocklehurst, M. 1971, MNRAS 153, 471

Cairós, L.M., Vílchez, J.M., González-Pérez, J.N., Iglesias-Páramo, J., 2000, submitted

Caldwell, N., Bothun, G.D., 1987, AJ 94, 1126

Calzetti, D., 1997, in "The Ultraviolet Universe at Low and High Redshift: Probing the Progress of Galaxy Evolution", W.H. Waller et al. (eds.), AIP conference proceedings, v. 408, 403

Campos-Aguilar, A., Moles, M., Masegosa, J., 1993, AJ, 106, 1784

Carignan, C., Beaulieu, S., 1989, ApJ 347, 760

Christian, C.A., Adams, M., Barnes, J.V., et al., 1985, PASP 97, 363

Davies, J.I., Phillips, S., 1988, MNRAS, 23, 553

De Young, D.S., Gallagher, J.S., 1990, ApJ 356, L15

De Young, D.S., Heckman, T.M., 1994, ApJ 431, 598

Dickinson, M., 1996, in "HST and the High Redshift Universe", N. Tanvir, A. Aragon-

Salamanca, J.V. Wall (eds.), World Scientific, London (astro-ph/9612178)

Dottori, H.M., Cepa, J., Vilchez, J.M., and Barth, C.S., 1994, A\&A 283, 753 
Doublier, V., Comte, G., Petrosian, A., et al., 1997, A\&AS 124, 405

Drinkwater, M., Hardy, E., 1991, AJ 101, 94

Drissen, L., Roy, J.R., Robert, C., Devost, D., Doyon, R., 2000, AJ 119, 688

Edmunds, M. G., Pagel, B. E. J. 1984, MNRAS 211, 507

Elmegreen, B.G., Elemgreen, D.M., Chromey, F.R., Hasselbacher, D.A., Bissel, B.A., 1996,

AJ 111, 2233

Ferland, G. J., 1980, PASP 92, 596

Freeman, K.C., 1970, ApJ 160, 811

Fricke, K.J., Papaderos, P., Izotov, Y.I., Thuan, T.X., 2000, in prep.

Garnett, D. R. 1992, AJ 103, 1330

Gil de Paz, A., Zamorano, J., Gallego, J., 1999, MNRAS 306, 975

González-Delgado, R.M., Pérez, E., Tenorio-Tagle, G., et al., 1994, ApJ 437, 239

Guseva, N.G., Izotov, Y.I., Thuan, T.X., 1998, Kinematics and Physics of Celestial Bodies $14, \mathrm{~N} 1,41$

Guseva, N.G., Izotov, Y.I., Thuan, T.X., 2000, ApJ, in press

Guzmán, R., Jangren, A., Koo, D.C., Bershady, M.A., Simard, L., 1998, ApJ 495, L13

Heckman, T.M. et al. 1998, ApJ 503, 646

Holtzman, J., et al., 1995a, PASP 107, 156

Holtzman, J., et al., 1995b, PASP 107, 1065

Hopp. U., Schulte-Ladbeck, R.E., 1991, A\&A 248, 1

HST Data Handbook, Update version 3.1, March 1998, Space Telescope Science Institute, available at: http://www.stsci.edu/documents/data-handbook.html

Hunter, D.A., Elemegreen, B.G., Baker, A.L., 1998, ApJ 493, 595

Hunter, D.A., Gallagher, J.S. III, 1997, AJ 475, 65

Izotov, Y.I., Dyak, A.B., Chaffee, F.H., Foltz, C.B., Kniazev, A.Y., Lipovetsky, V.A., 1996, ApJ 458, 524

Izotov, Y.I., Guseva, N.G., Lipovetsky, V.A., Kniazev, A.Y., Stepanian, J.A., 1990, Nature 343, 238

Izotov, Y.I., Papaderos, P., Thuan, T.X., Fricke, K.J., Foltz, C.B., Guseva, N.G., 1999, preprint (astro-ph/9907082)

Izotov, Y.I., Thuan, T.X., 1999, ApJ 511, 639

Izotov, Y.I., Thuan, T.X., Lipovetsky, V.A. 1994, ApJ 435, 647

Izotov, Y.I., Thuan, T.X., Lipovetsky, V.A. 1997, ApJS 108, 1

James, P.A., 1994, MNRAS 269, 176

Kaler, J.B., 1983, ApJ 264, 594

Kennicutt, R., Balick, B., Heckman, T, 1980, PASP 92, 134

Kinman, T.D., Davidson, K., 1981, ApJ 243, 127

Koo, D.C., Guzman, R., Gallego, J., 1997, ApJ 478, L49

Krüger, H., 1992, PhD Thesis, Universität Göttingen

Krüger, H., Fritze-v.Alvensleben, 1994, A\&A 284, 793

Krüger, H., Fritze-v.Alvensleben, U., Loose, H.-H., 1995, A\&A 303, 41

Kunth, D., Maurogordato, S., Vigroux, L., 1988, A\&A 204, 10

Kunth, D., Sargent, W.L.W., 1986, ApJ 300, 496

Lejeune, T., Cuisinier, F., Buser, R., 1998, A\&AS 130, 65

Lipovetsky, V.A., Chaffee, F.H., Izotov, Y.I., et al., 1999, ApJ 519, 177

Lisenfeld, U., Ferrara, A., 1998, ApJ 496, 145

Loose, H.-H., Thuan, T.X., 1985, in "Star-Forming Dwarf Galaxies and Related Objects",

Kunth, D., Thuan, T.X., Van, T.T. (eds.). Paris: Editions Frontières, p.73 (LT85)

Loose, H.-H., Thuan, T.X., 1986, ApJ 309, 59 
Mac Low, M.-M., Ferrara, A., 1999, ApJ 513, 142

Marlowe, A.T., Heckman, T.M., Wyse, R.F.G., Schommer, R., 1995, ApJ 438, 563

Marlowe, A.T., Meurer, G.R., Heckman, T.M., 1999, ApJ 522, 183

Marlowe, A.T., Meurer, G.R., Heckman, T.M., Schommer, R., 1997, ApJS 112, 285

Mas-Hesse, J.M., Kunth, D., 1999, A\&A 349, 765

Méndez, D.I., Cairós, L.M., Esteban, C., Vílchez, J.M., 1999, AJ 117, 1688

Meurer, G.R., 1998, astro-ph/9806304

Murakami, I., Babul, A., 1999, MNRAS 309, 161

Noeske, K.G., 1999, Diploma Thesis, Universität Göttingen

Noeske, K.G., Cairós Barreto, L.M., Papaderos, P., Vílchez, J.M., Fricke, K.J., 1999a, in "The Evolution of Galaxies on Cosmological Timescales", J. Beckman and T. Mahoney (eds.), ApSS (in press)

Noeske, K.G., Papaderos, P., Fricke, K.J., Thuan, T.X., 1998, in "The Magellanic Clouds and Other dwarf Galaxies", Braun, J.M., Richtler, T. (eds.). Bonn: Shaker Verlag, p.279

Papaderos, P., 1998, PhD Thesis, Universität Göttingen

Papaderos, P., Fricke, K.J. 1998, in the proc. of "Highlights in X-ray Astronomy", Garching 1998, B. Aschenbach et al. (eds.), in press (astro-ph/9810101)

Papaderos, P., Izotov, Y.I., Fricke, K.J., Thuan, T.X., Guseva, N.G., 1998, A\&A 338, 43

Papaderos, P., Loose, H.-H., Fricke, K.J., Thuan, T.X., 1996b, A\&A 314, 59 (P96b)

Papaderos, P., Loose, H.-H., Thuan, T.X., Fricke, K.J., 1996a, A\&AS 120, 207 (P96a)

Patterson, R.J., Thuan, T.X., 1996, ApJS 107, 103

Pustilnik, S.A., Thuan, T.X., Brinks, E., Lipovetsky, V.A., Izotov, Y.I., 1999, in prep.

Richter, G.M., Lorenz, H., Bohm, P., Priebe, A., 1991, Astron.Nachr. 312, 345

Roy, J.R., Boulesteix, J., Joncas, G., Grundseth, B., 1991, ApJ 367, 141

Salzer, J.J., MacAlpine, G.M., Boroson, T.A., 1989, ApJS 70, 479

Salzer, J.J., Norton, S.A., 1998, in: IAU Coll. 171, in press (astro-ph/9810338)

Sandage, A., Tammann, G.A., 1976, ApJ 210, 7

Sargent, W.L.W., Searle, L., 1970, ApJ 162L, 155

Savage, B.D., Mathis, J.S., 1979, ARA\&A 17, 73

Schaerer, D., Vacca, W. D. 1998, ApJ 497, 618

Simpson, C.E., 1995, PhD Thesis, Univ. of Florida

Stasińska, G., 1990, A\&AS 83, 501

Strickland, D.K., Stevens, I.R., 1999, MNRAS 306, 43

Swaters, R., 1998, in 'Dwarf Galaxies and Cosmology', eds. T.X. Thuan, C. Balkowski, V. Cayatte, J. Tran Thanh Van, in press

Taylor, C.L., Brinks, E., Pogge, R.W., Skillman, E.D., 194, AJ 107, 971

Telles, E., 1995, PhD Thesis, Cambridge

Telles, E., Terlevich, R., 1997, MNRAS 286, 183

Terlevich, R., Melnick, J., Masegosa, J., Moles, M., Copetti, M.V.F., 1991, A\&AS 91, 285

Thuan, T.X., 1985, ApJ 299, 881

Thuan, T.X., Izotov, Y.I., 1997, ApJ 489, 623

Thuan, T.X., Izotov, Y.I., Foltz, C.B., 1999, ApJ 525, 105

Thuan, T. X., Izotov, Y. I., Lipovetsky, V. A., 1995, ApJ 445, 108

Thuan, T.X., Izotov, Y.I., Lipovetsky, V.A., 1997, ApJ 477, 661

Thuan, T.X., Williams, T.B., Malumuth, E., 1987, in "Starbursts and Galaxy Evolution", T.X. Thuan, T. Montmerle, J.T. Thanh Van (eds.), Editions Frontières

Tolstoy, E., Saha, A., Hoessel, J.G., McQuade, K., 1995, AJ 110, 1640

Tully, R.B., 1988, "Nearby galaxies catalog", Cambridge Univ. Press

Vader, J.P., 1986, ApJ 317, 128 
van Zee, L., Salzer, J.J., Haynes, M.P., O’Donoghue, A.A., Balonek, T.J., 1998a, AJ 116, 2805

van Zee, L., Skillman, E.D., Salzer, J.J., 1998b, AJ 116, 1186

van Zee, L., Westphal, D., Haynes, M.P., Salzer, J.J., 1998c, AJ 115, 1000

Vigroux, L., Thuan, T.X., Vader, J.P., Lachièze-Rey, M., 1986, AJ 91, 70

Wevers, B.M.H.R., van der kruit, P.C., Allen, R.J., 1986, A\&AS 66, 502

Wilcots, E.M., Lehman, C., Miller, B., 1996, AJ 111, 1575 



\title{
The Environment of Star-Forming Dwarf Galaxies ${ }^{0}$ The Role Of Dwarf Companion Objects
}

\author{
K.G. Noeske,J. Iglesias-Páramo, J.M. Vílchez, K. J. Fricke \\ ${ }^{1}$ Universitäts-Sternwarte Göttingen \\ ${ }^{2}$ Instituto de Astrofísica de Canarias, Tenerife, Spain \\ ${ }^{3}$ Summer Research Student, Instituto de Astrofísica de Canarias
}

Summary. We present a new analysis of the close environment of star-forming dwarf galaxies and its effects on their star-forming activity. For a sample of 142 thoroughly studied objects, we search the NASA Extragalactic Database for possible companion galaxies of any apparent luminosity and angular size. From a first analysis of a part of our sample, we find only about 50\% of our objects to be isolated in the sense that no companion object was detected within a redshift difference of $500 \mathrm{kms}^{-1}$ and an angular separation corresponding to a projected distance of $1 \mathrm{Mpc}$, assuming $\mathrm{H}_{0}=75 \mathrm{kms}^{-1} \mathrm{Mpc}^{-1}$. By splitting the sample into different redshift intervals, we demonstrate an observational bias against low-luminosity companion objects which implies that a large fraction of star-forming dwarf galaxies, although lacking bright companions, might possess dwarf companion objects.

Analogous to other authors, we find no evidence for a difference of the star-forming activity due to the presence or absence of possible companions.

\section{V.1 Introduction}

Violent star formation in dwarf galaxies is among the physical processes still waiting to be explained by a commonly accepted model. Dwarf galaxies undergoing star formation (SF) at high rates are blue, gas-rich low-mass objects, usually classified as HII-galaxies (Terlevich et al. 1991), or Blue Compact Dwarf Galaxies (BCDs) (Thuan $\&$ Martin 1981). To investigate the phenomenon as a whole rather than restricting ourselves to one classification differing from another by slightly different criteria, we will consider the entire class of objects, referring to them as star-forming dwarf galaxies (SFDGs, cf. Vílchez 1995).

Being the principal driver of galaxy evolution, SF in dwarf galaxies has been the topic of much preceding work, focusing on the unknown mechanisms of its triggering and regulation, as well as its impact on the hosting dwarf galaxy. Among different suggestions, interaction with the SFDG's environment has been put forward to explain the massive SF. Consequently, samples of SFDGs were searched for possible companion

\footnotetext{
${ }^{0}$ This paper has been published in this form in Astrophysics \& Space Science, Volume 276, p. 577
} 
objects (Campos-Aguilar \& Moles 1991, Campos-Aguilar et al. 1993, Telles \& Terlevich 1995, see Vílchez 1997 for a review). The general results showed at least 2/3 of all SFDGs to be isolated, while only a small percentage of the objects had a massive companion in their vicinity. Objects displaying an irregular morphology and higher SF rates, properties generally considered to be signs of interaction, were surprisingly found to be devoid of any bright companions. The authors concluded that the triggering and regulation mechanisms of massive SF are solely based on the SFDG's internal processes, usually not depending on the presence of a luminous galaxy in the vicinity.

Vílchez $(1995,1997)$ compared spectroscopic properties of samples of SFDGs chosen from extremely high- and low-density environments. Objects in low-density environments showed higher star formation indicators than the ones from high-density environments. It seems, hence, that the SF activity can be lowered by environmentinduced effects, such as tidal shaking or stripping, or by ram-pressure stripping, processes expected to occur in environments with an enhanced density of galaxies and intergalactic medium, i.e. clusters or groups of galaxies.

Taylor et al. $(1995,1996)$ found 12 out of 21 observed HII-galaxies to possess lowmass HI-companion objects possibly capable of triggering SF. In a fraction of SFDGs lacking a bright companion, massive SF could therefore alternatively be induced by tidal distortions of close low-mass companion objects.

An analysis of the spatial distribution of galaxies in voids by Lindner et al. (1996) further supports the assumption of a considerable number of dwarf companion objects near SFDGs without bright companions. The results showed that practically all BCDs have close neighbors among dwarf galaxies, as long as the objects are at observable distances. Lindner et al. (1996) concluded that the observation of some distant BCDs which are apparently extremely isolated results from an observational bias effect against low-luminosity companion objects at large distances. BCDs were found to be either members of loose groups of dwarf galaxies, or to be situated in the peripheral regions of groups of bright galaxies.

Due to their low optical luminosity, dwarf companions are unlikely to be found by previous companion object searches. The CfA redshift catalog used by CamposAguilar et al. (1993) is limited at $13.5 \mathrm{mag}$, so at $20 \mathrm{Mpc}$ distance, an object with M> -18 mag will no longer be included. Telles \& Terlevich (1995) searched the NASA Extragalactic Database (NED) for bright $\left(\mathrm{M}_{B}<-19 \mathrm{mag}\right)$ companions only.

This work therefore aims at the study of a possible influence of objects with low optical luminosities on the SF in SFDGs, as well as a better understanding of the bias effects affecting such a study at large distances.

\section{V.2 Sample Selection}

To obtain a large sample of galaxies with uniformly available data, we included all dwarf emission-line galaxies from the University of Michigan Survey (UM) lists IV and V, for which spectra and BV CCD images were analyzed by Salzer et al. (1989a). To identify the SFDGs, we rejected all objects that were not classified as a 
dwarf or HIIH (HII-hotspot) galaxy by Salzer et al. (1989b). We furthermore took into account that our objects are currently undergoing strong SF, which raises the Bluminosity, e.g. by an average 0.75 mag for BCDs (Papaderos et al. 1996) compared to the quiescent state. Therefore, we extended the generally accepted brightness limit of $\mathbf{M}_{B}>-18$ mag for dwarf galaxies to $\mathbf{M}_{B}>-19$ mag to include objects which would possibly appear as a lower-luminosity galaxy during a quiescent interburst phase ( $\mathrm{Pa}-$ paderos et al. 1996).

More than $80 \%$ of these UM galaxies lie at redshifts $>2000 \mathrm{kms}^{-1}$, so we included a number of well-observed SFDGs from the Markarian, Zwicky, Haro, UGC and DDO catalogs to reach a better coverage of smaller object distances. Altogether, our sample contains 142 objects.

\section{V.2.1 The Long-Distance Sample}

To compare our results with the results of preceding work, which was mostly based on galaxies from the UM lists IV and V and therefore contains to a large part objects at redshifts $>2000 \mathrm{kms}^{-1}$, we selected a long-distance sample (LD-sample), consisting of 90 galaxies with redshifts $\geq 2000 \mathrm{kms}^{-1}$.

The short-distance sample, containing the remaining galaxies with redshifts $<2000 \mathrm{kms}^{-1}$, is currently being processed and compared to the long-distance one; the complete results, especially in terms of observational biases against low-luminosity companions, will be presented in a subsequent paper.

\section{V.3 Companion Search, Criteria for Isolation and Interaction}

We searched the NASA Extragalactic Database (NED) for possible companions of our sample objects. This database is frequently updated using newly published data and hence provides one of the most complete online-searchable lists of extragalactic objects detected at any wavelength.

To exclude possible tidal interactions with companion objects, we followed the estimations presented by Campos-Aguilar et al. (1993). Therefore, we considered a galaxy isolated if no objects were found within a redshift difference $\Delta v_{r}=500 \mathrm{kms}^{-1}$ and an angular distance corresponding to a projected separation of $d_{p}=1 \mathrm{Mpc}$, calculated from the redshift assuming a Hubble constant of $\mathrm{H}_{0}=75 \mathrm{kms}^{-1} \mathrm{Mpc}^{-1}$. Generally, all objects found within these limits were considered companion objects.

On the other hand, a galaxy was considered to be interacting if at least one companion within $d_{p}=0.1 \mathrm{Mpc}$ and $\Delta v_{r}=250 \mathrm{kms}^{-1}$ was present, separations typically found in the literature for pairs of galaxies (Laurikainen et al. 1998).

All companions with $\mathrm{M}_{B}>-18$ mag were classified as dwarf companions. To be able to compare our search results with previous work, we used the luminosity limit generally applied to dwarf galaxies instead of the extended one $\left(\mathrm{M}_{B}>-18 \mathrm{mag}\right)$ we describe in sect. 2 . 


\section{V.4 First Results}

According to the information found in the the NED, $80 \%$ of the LD-sample $\left(v_{r} \geq\right.$ $2000 \mathrm{kms}^{-1}$ ) was not found to belong to a pair, group or cluster. Our preliminary analysis showed that, according to our criteria, $48 \%$ of the LD-sample objects are isolated, while only $9 \%$ are likely to be a part of an interacting system.

The results of the companion search are listed in Table V.1.

The "reduced" subsamples 1-3 were extracted from the non-isolated subsample to do a first check for possible biasing effects against dwarf companions with rising distance, and contains the LD-sample galaxies within upper redshift limits of 8000 , 6000 and $4000 \mathrm{kms}^{-1}$, respectively. As can be seen from Table V.1, the fraction of dwarf galaxies among the found companions rises for subsamples that are restricted to smaller limiting redshifts.

Fig. V. 1 shows the distribution of $(\mathrm{B}-\mathrm{V})$-colors and $\mathrm{H} \beta$ equivalent widths among the galaxies of the LD-sample, to apply a first test whether the presence of a companion object can possibly influence the star-forming activity of a SFDG. Wilcoxon rank-sum tests to compare the distribution means of the different subsamples revealed no significant differences. The systematically bluer (B-V)-colors of the interacting galaxies are not significant because of the low number of objects contributing to the statistics.

\section{V.5 Discussion}

\section{V.5.1 Environment and Bias Effects against Dwarf Companions}

About $50 \%$ of our LD-sample is composed of candidate isolated galaxies. This value is smaller than the one deduced by Campos-Aguilar et al. (1993), although our LDsample is restricted to large redshifts. This is probably a result of the different search catalogs applied, with the NED generally reaching to fainter apparent magnitudes than the CfA catalog (Telles \& Terlevich 1995). Despite the varying completeness limits of the galaxy catalogs included in the NED, it can easily be argued that most of the dwarf

\begin{tabular}{lccc}
\hline & $\begin{array}{c}\text { Number of } \\
\text { galaxies }\end{array}$ & $\begin{array}{c}\text { Total } \\
\text { companions }\end{array}$ & $\begin{array}{c}\text { \% dwarf } \\
\text { companions }\end{array}$ \\
\hline Non-isolated subsample & 43 & 106 & 21.5 \\
$\begin{array}{l}\text { Reduced sample 1 } \\
\left(z<8000 \mathrm{kms}^{-1}\right)\end{array}$ & 41 & 104 & 22 \\
$\begin{array}{l}\text { Reduced sample 2 } \\
\left(z<6000 \mathrm{kms}^{-1}\right)\end{array}$ & 24 & 65 & 26 \\
$\begin{array}{l}\text { Red. sample 3 } 3 \\
\left(z<4000 \mathrm{kms}^{-1}\right)\end{array}$ & 10 & 33 & 36.5 \\
\hline Interacting subsample & 8 & 25 & 20 \\
\hline
\end{tabular}

Table V.1. Companion search for the long-distance sample 

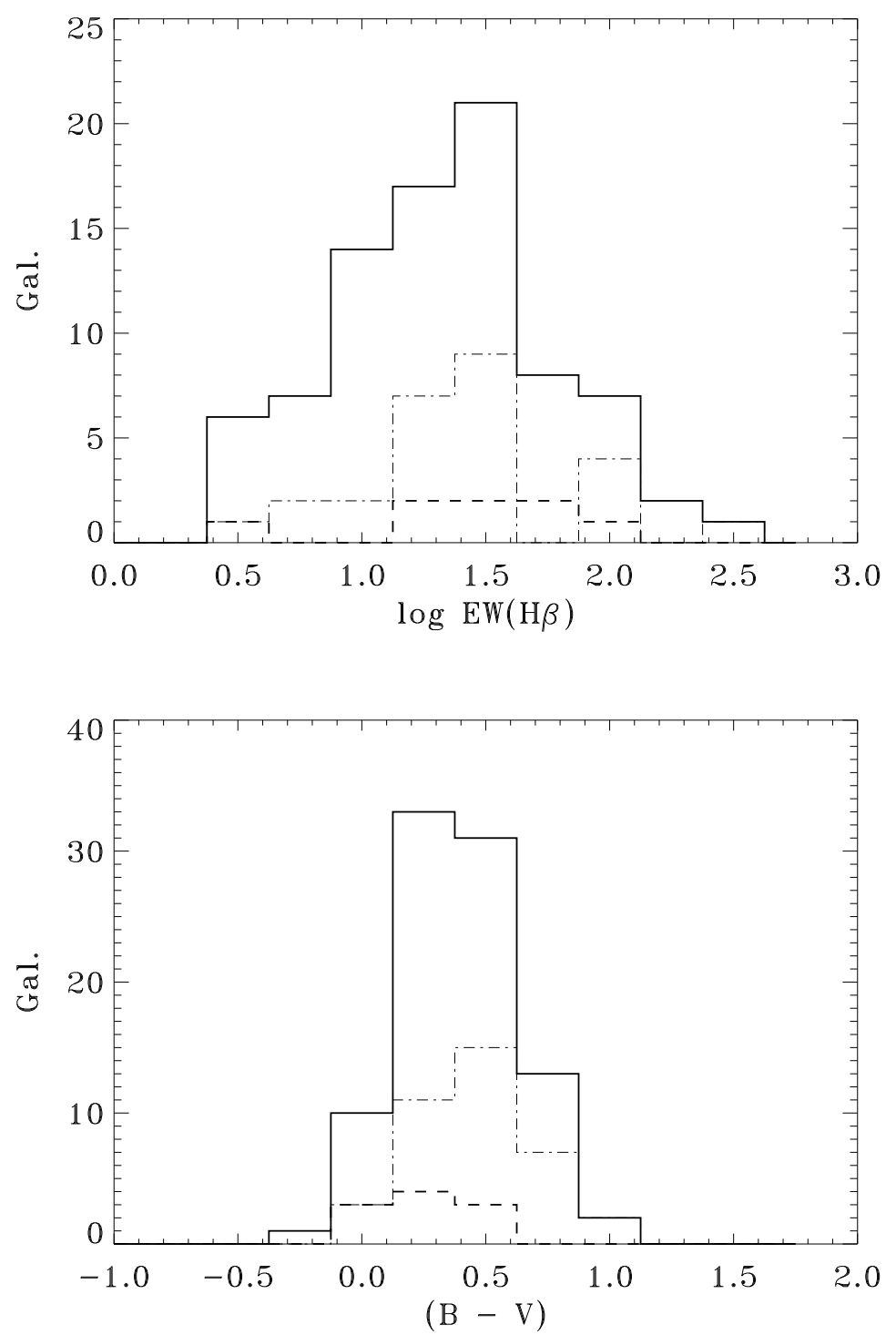

Fig. V.1. Histograms for the distributions of the $\mathrm{H} \beta$ equivalent widths (top) and (B-V) colors, corrected for galactic foreground extinction (bottom) of the long-distance sample galaxies. Continuous lines: total long-distance sample, dashed lines: interacting galaxies, dot-dashed lines: isolated galaxies.

companion objects have been missed. If a catalog's limiting magnitude is $16 \mathrm{~B}$ mag, an object at $40 \mathrm{Mpc}$ distance must be brighter than $-17 \mathrm{~B}$ mag to be included, whereas the luminosities of typical dwarf galaxies reach down to -12 B mag. As our search criteria require not only the detection in a photometric survey, but also a known redshift to regard a galaxy as a possible companion, the probability to find a low-luminosity, low-surface brightness galaxy with a small spatial extent is lowered even further. It is therefore justified to assume that considerably more than $50 \%$ of the SFDGs are probably not isolated according to our criteria, but possess low-luminosity companions. 
The bias effect shown in Table V.1 will be further studied using the data of the short-distance galaxy sample.

\section{V.5.2 Environmental Effects on Star Formation}

Analogous to the results of other authors, our data yield no evidence for a change in the SF activity in the presence or absence of a possible companion galaxy. This further supports the hypothesis that triggering and regulation of SF in SFDGs is mostly based on internal processes rather than interaction, although for some cases interactions are known to be the main agents that trigger SF (Iglesias-Páramo \& Vílchez 1997).

We note that the intrinsic scatter of spectrophotometric properties among SFDGs is quite large (cf. Salzer et al. 1989a, 1989b). The detection of small systematic differences between different subsamples of galaxies is therefore rendered difficult.

The $\mathrm{H} \beta$ equivalent width, for example, may not be the most appropriate tracer for the general influence of one or more near possible companions on the SF activity. Once triggered, SF in dwarf galaxies generally takes place in short events of $\lesssim 10^{7} \mathrm{yr}$ (Elmegreen et al. 1996), followed by longer quiescent phases (cf. Thuan 1991). The $\mathrm{H} \beta$-equivalent width should therefore be enhanced on comparatively short time scales only, due to the short lifetimes of the newly-formed ionizing stars (cf. the models by Leitherer \& Heckman 1995), hence depending not only on possible environmental influences, but also strongly on the age of the last event of SF. To overcome this dependence, statistics with very large samples are necessary.

Our future work will include the investigation of the short-distance sample, where a greater fraction of the dwarf companions should be detected, to further study a possible influence of low-luminosity galaxies on a SFDG's SF activity.

Acknowledgement. We thank P. Papaderos for helpful suggestions. This work received financial support from the IAC Summer Research Program 1998. We have made use of the NASA/IPAC Extragalactic Database (NED) which is operated by the Jet Propulsion Laboratory, CALTECH, under contract with the National Aeronautic and Space Administration.

\section{References}

Campos-Aguilar, A., Moles, M., 1991, A\&A, 241, 358

Campos-Aguilar, A., Moles, M., Masegosa, J., 1993, AJ, 106, 1748

Dultzin-Hacyan, D., Masegosa, J., Moles, M., 1990, A\&A, 238, 28

Elmegreen, B.G., Elmegreen, D.M., Salzer, J.S., Mann, H., 1996, ApJ, 467, 579

Iglesias-Páramo, J., Vílchez, J.M., 1997, ApJ, 479, 190

Laurikainen, E.,. Salo, H., Aparicio, A., 1998, A\&AS, 129, 517

Leitherer, C., Heckman, T.M., 1995, ApJS 96, 9

Lindner, U., Einasto, M., Einasto, J., Freudling, W., Fricke, K.J., Lipovetsky, V., Pustilnik, S., Izotov, Y., Richter, G., 1996, A\&A, 314, 1

Papaderos, P., Loose, H.-H., Fricke, K.J., and Thuan, T.X., 1996, A\&A, 314, 59

Salzer, J.J., MacAlpine, G.M., Boronson, T.A., 1989a, ApJS, 70, 447

Salzer, J.J., MacAlpine, G.M., Boronson, T.A., 1989b, ApJS, 70, 479 
Taylor, C.L., Brinks, E., Grashius, R.M., Skillman, E.D., 1995, ApJS, 99, 427

Taylor, C.L., Brinks, E., Grashius, R.M., Skillman, E.D., 1996, ApJS, 102, 189

Telles, E., Terlevich, R., 1995, MNRAS, 275, 1

Terlevich, R., Melnick, J., Masegosa, J., Moles, M., Copetti, M.V.F., 1991, A\&AS, 91, 885

Thuan, T.X., 1991, Massive Stars in Starbursts, eds. C. Leitherer, N.R. Walborn, T.M. Heckman, C.A. Norman, Cambridge University Press, 183

Thuan, T.X., Martin, G.E., 1981, ApJ, 243, 823

Vílchez, J.M., 1995, AJ, 110, 1090

Vílchez, J.M., 1997, RevMexAA (Serie de Conferencias), 6, 30 



\title{
On faint companions in the close environment of star-forming dwarf galaxies ${ }^{0}$ Possible external star formation triggers?
}

\author{
K.G. Noeske ${ }^{1,4}$, J. Iglesias-Páramo ${ }^{2}$, J.M. Vílchez ${ }^{3}$, P. Papaderos ${ }^{1}$, K.J. Fricke ${ }^{1}$ \\ ${ }^{1}$ Universitäts-Sternwarte Göttingen, D-37083 Göttingen, Germany \\ ${ }^{2}$ Instituto de Astrofísica de Canarias, 38200 La Laguna, S/C de Tenerife, Spain \\ ${ }^{3}$ Instituto de Astrofísica de Andalucía (CSIC), 18080 Granada, Spain \\ ${ }^{4}$ Summer research student 1998, Instituto de Astrofísica de Canarias
}

Summary. We have searched for companion galaxies in the close environment of 98 starforming dwarf galaxies (SFDGs) from field and low density environments, using the NASA Extragalactic Database. Most of the companions are dwarf galaxies which due to observational selection effects were previously disregarded in environmental studies of SFDGs. A subsample at low redshift, $c z<2000 \mathrm{~km} \mathrm{~s}^{-1}$, was chosen to partially eliminate the observational bias against distant dwarf companions. We find companion candidates for approximately $30 \%$ of the objects within a projected linear separation $s_{p}<100 \mathrm{kpc}$ and a redshift difference $\Delta c z<500 \mathrm{~km} \mathrm{~s}^{-1}$. The limited completeness of the available data sets, together with the nonnegligible frequency of H I clouds in the vicinity of SFDGs indicated by recent radio surveys, suggest that a considerably larger fraction of these galaxies may be accompanied by low-mass systems. This casts doubt on the hypothesis that the majority of them can be considered truly isolated. The velocity differences between companion candidates and sample SFDGs amount typically to $\lesssim 250 \mathrm{~km} \mathrm{~s}^{-1}$, and show a rising distribution towards lower $\Delta c z$. This is similarly found for dwarf satellites of spiral galaxies, suggesting a physical association between the companion candidates and the sample SFDGs. SFDGs with a close companion do not show significant differences in their $\mathrm{H} \beta$ equivalent widths and $B-V$ colours as compared to isolated ones. However, the available data do not allow us to rule out that interactions with close dwarf companions can influence the star formation activity in SFDGs.

\section{VI.1 Introduction}

Dwarf galaxies are considered important contributors to the baryonic mass of the Universe and to the star formation rate (SFR) density at higher redshifts (see. e.g. Guzmán et al. 1998). Some of them may even be similar to low-mass building blocks of normal galaxies in a bottom-up cosmological model. A better understanding of the processes that drive their evolution is therefore central to cosmological studies, and to the understanding of galaxy formation and evolution.

\footnotetext{
${ }^{0}$ This paper has been published in this form in Astronomy \& Astrophysics, Volume 371, p. 756
} 
Starbursts, i.e. brief episodes of strongly enhanced star formation (SF) activity, are thought to occur frequently during the lifetime of a gas-rich dwarf galaxy. A number of internal processes has been put forward to explain their origin and transient nature, such as Stochastic Self-Propagating Star Formation (Gerola, Seiden \& Schulmann 1980, see Thuan 1991) or a cyclic process of gas infall onto and expulsion from an older stellar host (e.g. Davies \& Phillips 1988, Papaderos et al. 1996).

Alternatively, environmental influences have been suggested to induce starburst activity in star-forming dwarf galaxies (SFDGs; see Section VI.2). Based on the hypothesis that dwarf galaxies are affected in a similar way to luminous disk galaxies (e.g. Kennicutt et al. 1987), mainly tidal interactions with stellar or gaseous companions have been treated by former studies.

Information on the presence and frequency of such external perturbors has been assembled from environmental studies of emission line galaxies (ELGs), performed mainly in the context of large scale structure formation and biased formation and evolution of galaxies in different environments. ELGs were found to smoothly follow the structures delineated by luminous galaxies, although they are more fuzzily distributed (Rosenberg et al. 1994), generally populating lower-density environments (Salzer 1989, Telles \& Terlevich 1995, hereafter TT95), and are less strongly clustered than less active luminous galaxies (Loveday et al. 1999, Telles \& Maddox 1999, Lee et al. 2000). As for SFDGs, they were found to be concentrated towards void boundaries (Lindner et al. 1996, hereafter L96), with a fraction $(\approx 20 \%)$ of them inside voids (Pustilnik et al. 1995), arranged in loose groups which do not contain any bright galaxies (L96). Interactions with luminous companions were therefore considered too rare to be the generic triggering agent of starbursts in SFDGs, given that luminous galaxies are rare in the close neighbourhood of SFDGs.

Whether interactions with companion galaxies can significantly influence SF activity and evolution of SFDGs has been investigated in earlier work (Campos-Aguilar et al. 1991, 1993; TT95). The spectrophotometric properties of the studied objects showed no significant dependence on the presence or absence of a companion galaxy, and both SF activity and metallicity seemed unrelated to distance or absolute magnitude of putative companions. Campos-Aguilar et al. chose their samples from the spectrophotometric catalogue of H II galaxies (SCHG, Terlevich et al. 1991), which mostly covers redshifts $c z \gtrsim 2000 \mathrm{kms}^{-1}$. Taking into account the magnitude cutoff of the CfA catalogue used for the companion search (13.5 mag), these studies were practically restricted to luminous companions ( $\mathrm{M} \lesssim-18 \mathrm{mag}$ ), typically not particularly close to SFDGs. TT95, on their part, explicitly focus on the possible influence of luminous companions $(\mathrm{M}<-19 \mathrm{mag})$. Also, the opposite conclusion by Grogin \& Geller (2000), that ELGs in low-density environments show enhanced SF activity in the presence of a companion, is mostly drawn from studies of luminous ELGs and companions.

Observational evidence of a different kind points towards a correspondence between the membership of a SFDG in a given environment and its spectrophotometric properties. A comparative study of SF activity in SFDGs populating different environments by Vílchez $(1995,1997)$ suggests that objects in low density environments have 
higher SF activity than those located in high density regions. The results of Hashimoto et al. (1998) point in the same direction. Popescu, Hopp and Rosa (1999) reported no conspicuous difference in present and past SF activity for ELGs in field and void environments. On the other hand, Vennik, Hopp and Popescu (2000) found that the low surface brightness hosts of non-isolated ELGs tend to be more compact than those of isolated ones.

The mixed evidence for an influence of the environment on the SF activity of a SFDG calls for further studies, extending previous ones to larger samples and fainter magnitudes. This seems particularly important in view of the conjecture by L96, who attributed the apparent isolation of a fraction of BCDs to an observational bias against their low-luminosity companions.

Assuming that companions are capable of inducing SF activity in SFDGs, the mechanism and the prime parameters controlling this process remain unclear. Tidal forces are, however, considered to be of major importance. If $D_{c}$ and $M_{c}$ denote respectively the distance and mass of a companion, the tidal forces acting on a SFDG scale as

$$
F_{t i d} \propto M_{c} \times D_{c}^{-3}
$$

so that a nearby low-mass, i.e. faint, perturbor can affect a SFDG to the degree a more distant giant galaxy does (cf. Campos-Aguilar et al. 1991). Observational data (Wilcots et al. 1996) and numerical models (Hensler et al. 1999, Pilyugin 2000) further suggest that, in addition to tidal interactions, low-mass stellar or gaseous companions may trigger and fuel starbursts by infalling onto a SFDG.

Although Telles \& Maddox (1999), from an analysis of APM catalogues, found no excess of dwarf companions for $\mathrm{H}$ II galaxies down to $\approx-14.5 \mathrm{~B}$ mag and $\sim 10^{8}$ $\mathrm{M}_{\odot}$, numerous results point to the presence of objects of even lower masses and luminosities. Non-catalogued objects of low optical luminosities and surface brightnesses were found for most one-armed magellanic irregulars (Odewahn 1994), and around many BCDs (Pustilnik et al. 1997, Walter et al. 1997, Doublier et al. 1999, Méndez \& Esteban 1999).

Likewise, H I observations of the environment of magellanic irregulars (Wilcots et al. 1996) and H II galaxies (Taylor et al. 1994, 1995, 1996) revealed H I companions for the majority of them, down to intergalactic $\mathrm{H}$ I clouds with masses of $\sim 10^{7} \mathrm{M}_{\odot}$ for some of which no optical counterpart was found.

The presence of still undetected objects of low optical and H I luminosity appears also likely, keeping in mind that the LF steepens towards the low-luminosity end for SFDGs (Loveday et al. 1999), and in view of e.g. the high space density and the masses of local Ly $\alpha$ absorbers (Shull et al. 1996). Grogin \& Geller (2000) found for luminous ELGs that the frequency of companions at low redshift differences is widely independent of the local galaxy density. Therefore, faint companions may not be rare even in regions of low density of luminous galaxies.

The aim of this study is to investigate the incidence of close optical companions of SFDGs and whether such close companions strongly affect a SFDG's SF activity. Particular attention is attached to faint close companions, the presence and influence of which was generally not assessed in previous work. In order to address the problem of 
observational bias against low-luminosity sources, we investigate separately a subset of nearby SFDGs with recession velocities $<2000 \mathrm{kms}^{-1}$. For a sample of SFDGs, we compiled photometric and spectroscopic properties at different wavelengths and corrected distance dependent observables for the Virgocentric infall.

The paper is organized as follows: In Section VI.2 we describe our galaxy sample and the data processing. In Section VI.3, we analyse our data sets by applying different statistical methods and list the results, which are further discussed in Section VI.4. In Section VI.5, we summarize our work and conclusions. We assume $H_{0}=75 \mathrm{~km} \mathrm{~s}^{-1} \mathrm{Mpc}^{-1}$ throughout.

\section{VI.2 The Data}

\section{VI.2.1 Sample selection}

Various criteria were applied to select samples of dwarf galaxies with current or recent strong SF, which resulted in catalogues of Blue Compact Dwarf Galaxies (BCDs, e.g. Thuan \& Martin 1981) and H II galaxies (e.g. SCHG, Terlevich et al. 1991). Despite the different classification schemes, it has been subsequently shown that the underlying stellar hosts of starbursting dwarf galaxies have generally a compact structure (Papaderos et al. 1996, Marlowe et al. 1997, Salzer \& Norton 1998). Also comparative studies of dwarf H II galaxies, BCDs and dwarf amorphous galaxies with ongoing SF showed them to widely share the colours and structural properties of their host galaxies, relative starburst luminosities and $E W(\mathrm{H} \alpha)$, suggesting that they basically form one and the same class of extragalactic objects (Papaderos et al. 1996, Telles et al. 1997, Marlowe et al. 1999). We will therefore unify both BCDs and H II galaxies using the term "star-forming dwarf galaxies" (SFDGs), following Vílchez (1995, 1997).

In order to study a sufficiently large SFDG sample for which comparable observational data are available, we decided to use lists IV and V of the sample of ELGs from the University of Michigan (UM) Survey (McAlpine et al. 1977a, 1977b, 1977c; McAlpine \& Lewis 1978; McAlpine \& Williams 1981), systematically investigated in Salzer et al. (1989a, 1989b) by means of spectroscopy and CCD imaging in $B$ and $V$. Among the different types of ELGs, all objects classified as $\mathrm{H}$ II galaxies were selected; the magnitude limit of $\mathrm{M}_{B}>-18 \mathrm{mag}$, commonly adopted to select dwarf galaxies, was applied after the distance correction (Section VI.2.3) had been performed. More than $80 \%$ of these UM galaxies are located at redshifts $c z>2000 \mathrm{~km} \mathrm{~s}^{-1}$.

Our sample further includes SFDGs studied by Cairós and Noeske (cf. Cairós \& Vílchez 1998, Cairós et al. 2000, Noeske et al. 1998a, 1998b, Noeske 1999). These are, for the most part, nearby objects $\left(c z<2000 \mathrm{~km} \mathrm{~s}^{-1}\right)$ which allow an environmental search that is less biased against faint objects than the quite distant UM sample. None of our sample galaxies are members of galaxy clusters. Those which are known or likely members of groups (cf. NED and Garcia 1993) were rejected (Mkn 35, Mkn 71 and Mkn 527). Only 3 galaxies, UM 454, UM 455 and UM 513, have been reported to lie inside voids (Salzer 1989). The resulting sample consists of 98 dwarf galaxies in typical field- and low density environments and is listed in Table VI.1. 
Table VI.1. Sample list: (1) name; (2) recession velocity in $\mathrm{km} \mathrm{s}^{-1}$, corrected for peculiar velocities introduced by Virgo Cluster perturbations (see Section VI.2.3); (3) integral $B-V$ colour; (4) $\mathrm{H} \beta$ equivalent width (in emission); (5) number of close companion candidates found in the NED within the limits described in Section VI.3.1.1.

\begin{tabular}{lcccc}
\hline Name & $\begin{array}{c}c z^{\text {corr. }} \\
\mathrm{km} \mathrm{s}^{-1}\end{array}$ & $\begin{array}{c}B-V \\
\text { mag }\end{array}$ & $\begin{array}{c}E W(\mathrm{H} \beta) \\
\AA\end{array}$ & \# Comp. \\
& $(2)$ & $(3)$ & $(4)$ & $(5)$ \\
\hline UM 306 & 4985 & 0.42 & 26.4 & 0 \\
UM 92 & 6949 & 0.33 & 38.3 & 0 \\
UM 323 & 1998 & 0.38 & 25.9 & 0 \\
UM 330 & 5141 & 0.57 & 18.8 & 0 \\
UM 334 & 4924 & 0.32 & 11.4 & 0 \\
UM 335 & 4984 & 0.13 & - & 0 \\
UM 336 & 5816 & 0.24 & 54.9 & 0 \\
UM 345 & 5754 & 0.57 & 25.7 & 0 \\
UM 351 & 7487 & 0.55 & 44.5 & 0 \\
UM 369 & 5812 & 0.40 & 58.2 & 0 \\
UM 371 & 5536 & 0.49 & 6.5 & 0 \\
UM 372 & 1752 & 0.31 & 92.7 & 0 \\
UM 374 & 5735 & 0.59 & 34.6 & 0 \\
UM 379 & 8227 & 0.78 & 26.6 & 0 \\
UM 151 & 4890 & 0.46 & 9.8 & 0 \\
UM 382 & 3786 & 0.39 & 124.2 & 0 \\
UM 396 & 6208 & 1.00 & 123.5 & 0 \\
UM 404 & 3712 & 0.45 & 74.1 & 1 \\
UM 406 & 11251 & 0.92 & 104.1 & 0 \\
UM 408 & 3637 & 0.53 & 46.8 & 0 \\
UM 410N & 6960 & 0.62 & 35.4 & 1 \\
UM 411 & 11748 & 0.78 & 480.5 & 0 \\
UM 417 & 2846 & 0.19 & 63.6 & 0 \\
UM 422 & 2017 & 0.21 & 328.6 & 0 \\
UM 439 & 949 & 0.27 & 41.2 & 0 \\
UM 442 & 7707 & 0.41 & 24.8 & 0 \\
UM 444 & 6332 & 0.65 & 36.2 & 0 \\
UM 446 & 1418 & 0.45 & 34.8 & 0 \\
UM 452 & 1219 & 0.63 & 13.5 & 0 \\
UM 454 & 3592 & 0.42 & 21.4 & 0 \\
UM 455 & 3657 & 0.36 & 59.7 & 0 \\
UM 456 & 1518 & 0.34 & 42.7 & 0 \\
UM 461 & 735 & 0.43 & 241.2 & 1 \\
UM 462 & 855 & 0.44 & 72.6 & 1 \\
UM 463 & 1131 & 0.45 & 98.5 & 0 \\
UM 465 & 1155 & 0.59 & 15.7 & 1 \\
UM 471 & 10185 & 0.76 & 41.8 & 0 \\
UM 483 & 2099 & 0.92 & 27.7 & 0 \\
UM 487 & 14419 & 0.50 & 6.6 & 0 \\
UM 490 & 5376 & -0.15 & 113.0 & 0 \\
UM 491 & 1753 & 0.42 & 14.6 & 0 \\
UM 495 & 7477 & 0.52 & 47.7 & 0 \\
UM 496 & 11479 & 0.73 & 110.9 & 0 \\
\hline & & & &
\end{tabular}


Table VI.1. (continued)

\begin{tabular}{|c|c|c|c|c|}
\hline Name & $\begin{array}{c}c z^{\text {corr. }} \\
\mathrm{km} \mathrm{s}^{-1} \\
(2) \\
\end{array}$ & $\begin{array}{c}B-V \\
\operatorname{mag} \\
(3) \\
\end{array}$ & $\begin{array}{c}E W(\mathrm{H} \beta) \\
\AA \\
(4) \\
\end{array}$ & $\begin{array}{c}\text { \# Comp. } \\
\text { (5) }\end{array}$ \\
\hline UM 500 & 1805 & 0.15 & 117.8 & 1 \\
\hline UM 501 & 1745 & 0.39 & 184.5 & 1 \\
\hline UM 504 & 1867 & 0.50 & 17.9 & 0 \\
\hline UM 507 & 6094 & 0.58 & 94.2 & 0 \\
\hline UM 512 & 4488 & 0.51 & 13.8 & 0 \\
\hline UM 513 & 3457 & 0.82 & 7.3 & 0 \\
\hline UM 523 & 1620 & 0.51 & 16.9 & 1 \\
\hline UM 533 & 737 & 0.35 & 206.5 & 1 \\
\hline UM 538 & 855 & 0.56 & 41.2 & 0 \\
\hline UM 539 & 6065 & 0.60 & 56.5 & 0 \\
\hline UM 549 & 5642 & 0.36 & 15.5 & 0 \\
\hline UM 552 & 7190 & 0.48 & 20.9 & 0 \\
\hline UM 559 & 1037 & 0.18 & 261.7 & 0 \\
\hline UM 562 & 5290 & 0.91 & 199.5 & 0 \\
\hline UM 564 & 13668 & 0.86 & 101.1 & 0 \\
\hline UM 570 & 6543 & 1.18 & 199.2 & 0 \\
\hline UM 588 & 3581 & 0.47 & 18.6 & 1 \\
\hline UM 591 & 15642 & 0.61 & 38.6 & 0 \\
\hline UM 597 & 6441 & 0.46 & 22.7 & 0 \\
\hline UM 605 & 4956 & 0.59 & 6.1 & 0 \\
\hline UM 612 & 4414 & 0.54 & 19.1 & 0 \\
\hline UM 618 & 4188 & 0.39 & 39.5 & 0 \\
\hline UM 619 & 4455 & 0.63 & 10.4 & 0 \\
\hline UM 626 & 3335 & 0.57 & 5.1 & 0 \\
\hline UM 628 & 7052 & 0.15 & 32.3 & 0 \\
\hline UM 635 & 7192 & 0.47 & 25.9 & 0 \\
\hline UM 648 & 9715 & 0.78 & 4.4 & 0 \\
\hline UM 649 & 7554 & 0.58 & 81.7 & 0 \\
\hline UGC 47 & 873 & 0.34 & - & 1 \\
\hline TOL 0127-397 & 4797 & 0.59 & 38.0 & 0 \\
\hline DDO 19 & 735 & 1.21 & - & 0 \\
\hline MKN 370 & 840 & 0.63 & 10.0 & 0 \\
\hline MKN 600 & 945 & 0.46 & - & 0 \\
\hline UGC 2432 & 764 & 0.89 & - & 0 \\
\hline UGC 2482 & 2646 & 0.61 & - & 0 \\
\hline UGC 2809 & 1239 & 0.49 & - & 0 \\
\hline c0341-4045 & 4497 & 0.46 & - & 0 \\
\hline DDO 34 & 615 & -0.40 & - & 0 \\
\hline MKN 5 & 792 & 0.50 & 150.0 & 0 \\
\hline UGC 3516 & 1287 & 0.65 & - & 0 \\
\hline UGC 3658 & 1267 & 0.29 & - & 0 \\
\hline MKN 86 & 675 & 0.66 & 6.2 & 2 \\
\hline I Zw 18 & 1072 & 0.19 & 69.0 & 0 \\
\hline MKN 36 & 517 & 0.46 & 70.0 & 1 \\
\hline VII ZW 403 & 105 & - & - & 0 \\
\hline
\end{tabular}


Table VI.1. (continued)

\begin{tabular}{lcccc}
\hline Name & $\begin{array}{c}c z^{\text {corr. }} \\
\mathrm{km} \mathrm{s}^{-1} \\
(2)\end{array}$ & $\begin{array}{c}B-V \\
\mathrm{mag} \\
(3)\end{array}$ & $\begin{array}{c}E W(\mathrm{H} \beta) \\
\AA\end{array}$ & \# Comp. \\
& $(4)$ & $(5)$ \\
\hline NGC 4670 & 825 & 0.39 & - & 0 \\
MKN 209 & 352 & 0.53 & 60.3 & 2 \\
II Zw 70 & 1732 & 0.52 & 49.0 & 1 \\
II Zw 71 & 1785 & 1.23 & - & 1 \\
PHL 293 B & 1762 & 0.77 & 110.0 & 0 \\
MKN 324 & 1635 & 0.60 & 3.8 & 0 \\
MKN 67 & 1680 & 0.31 & 105.0 & 0 \\
MKN 169 & 1777 & 0.51 & 21.9 & 0 \\
I Zw 123 & 1125 & 0.60 & 144.5 & 0 \\
HARO 38 & 1327 & - & - & 0 \\
\hline
\end{tabular}

\section{VI.2.2 Search for companion objects}

Our companion search catalogue was the NASA Extragalactic Database ${ }^{1}$ (NED).

Most of our objects have declinations $\delta>-3^{\circ} 30^{\prime}$, i.e. lie within the sky region covered by the CGCG (Zwicky 1961), which is included into the NED (cf. TT95). We can therefore attribute the completeness limit of the CGCG, $\mathrm{m}_{B}^{\text {lim }} \approx \mathrm{m}_{\text {phot }}^{\text {lim }} \approx 15.5 \mathrm{mag}$, to our companion search. As the NED includes numerous other literature sources, considerably fainter objects should partly be detectable, which makes NED a suitable catalogue to find as many known faint sources as possible. The non-uniform completeness of this database does, on the other hand, not constitute a drawback since a meaningful extrapolation beyond even a well-defined completeness limit is at present not possible (see Section VI.4.1).

In an initial selection procedure, we considered any type of extragalactic source a possible companion. Statistical analyses of spatial companion frequencies from excess source densities around SFDGs have been previously done (Telles \& Maddox 1999). We hence restricted ourselves to sources for which redshift data was available. Thereby some companions are missed, but information on individual companions' properties such as luminosity etc. can be analyzed.

Any object within a projected separation $s_{p} \leq 0.1 \mathrm{Mpc}$ from our sample galaxies was included into a tentative list of companions. The corresponding angular search radius was calculated from the redshift $c z$ given in the NED, assuming a pure Hubble flow. We allowed, in the first place, a maximum separation in velocity space of $\Delta c z \leq$ $2000 \mathrm{~km} \mathrm{~s}^{-1}$ between sample object and companion. The final selection criterion below which we finally considered an object a companion was, however, $\Delta c z \leq 500 \mathrm{~km} \mathrm{~s}^{-1}$ (cf. Section VI.3.1.1).

\footnotetext{
${ }^{1}$ The NASA/IPAC Extragalactic Database (NED) is operated by the Jet Propulsion Laboratory, California Institute of Technology, under contract with the National Aeronautics and Space Administration.
} 


\section{VI.2.3 Distances and correction for Virgo Cluster infall}

The choice of a less distant subsample of SFDGs with $c z<2000 \mathrm{~km} \mathrm{~s}^{-1}$ does not justify the assumption of a pure Hubble flow throughout to determine galaxy distances, but requires a correction for the peculiar velocity field in the environment of the Virgo Cluster (VC). For this purpose, we adopted the distances listed in the Nearby Galaxies Catalog (Tully 1988, hereafter T88). These are based on a Virgocentric infall model, which uses redshift - independent distance determinations, morphology and neighbourhood considerations to treat those galaxies affected by the triple value problem (see Tully \& Shaya 1984).

For all objects not listed in T88, the distance was directly calculated from their redshift given in the NED. Galaxies within the redshift range covered by T88, $c z<3000 \mathrm{~km} \mathrm{~s}^{-1}$, which are not listed in the catalogue, were found to be at least 1.5 mag fainter than the dwarf - non dwarf separation limit of $\mathrm{M}_{B}>-18$ mag, i.e. can be considered dwarfs despite their possibly larger distance insecurities. For galaxies outside the redshift range of T88, the deviation of the true distance from the one obtained assuming a pure Hubble Flow can be estimated from Kraan-Korteweg (1986, cf. their Fig. 3b) to $\$ 30 \%$ due to VC infall effects. A comparable deviation was obtained by Marinoni et al. (1998, cf. their Fig. 14) who have adopted in their study a multiattractor model. The above mentioned dispersion translates in the worst case into an uncertainty of $\lesssim 0.6$ mag in the distance modulus which may then slightly affect the luminosity-based distinction between dwarf and luminous galaxies. This does not affect the distance-independent quantities $B-V$ and $E W(\mathrm{H} \beta)$ involved in our study.

For the companion candidates, we assumed the same distances as for the sample galaxies around which they were found. Given that the peculiar velocity field in the VC vicinity is not expected to change dramatically on scales of few tens of kpc, and that the companions show signs of physical association with the sample galaxies (cf. Section VI.3.1.1), the above assumption appears justified. For these distances, projected separations $s_{p}$ between sample objects and candidate companions were calculated from their angular separation.

\section{VI.2.4 Photometric and spectroscopic data}

Aperture photometry in optical broadbands was taken from the NED i.e. listing mostly RC3 data, from Salzer (1989a, 1989b), Cairós et al. (2000), Noeske (1999), and Noeske et al. (1998a, 1998b). H $\beta$ equivalent widths were compiled from French (1980), Thuan \& Martin (1981), the SCHG (Terlevich et al. 1991) and Salzer (1989a, 1989b).

Adopting the distances obtained as described in Section VI.2.3, apparent magnitudes were transformed to absolute values. A correction for galactic foreground dust extinction was applied to all photometric data using the $B$ band extinction given in the NED for each galaxy, and applying the standard reddening curve by Savage \& Mathis (1979). 
Table VI.2. Companion search results for subsamples within different redshift intervals

\begin{tabular}{|c|c|c|c|c|}
\hline $\begin{array}{l}c z^{a} \\
\text { range } \\
\text { (1) } \\
\end{array}$ & $\begin{array}{c}\text { sample } \\
\text { size } \\
(2) \\
\end{array}$ & $\begin{array}{c}\text { non } \\
\text { isol. } \\
(3)\end{array}$ & $\begin{array}{c}\text { no. of } \\
\text { comps. } \\
(4)\end{array}$ & $\begin{array}{c}\text { dwarf } \\
\text { comps. } \\
(5)\end{array}$ \\
\hline unconstrained & 98 & 16 & 18 & 15 \\
\hline$c z<2000$ & 42 & 13 & 15 & 14 \\
\hline $2000 \leq c z<4000$ & 12 & 2 & 2 & 1 \\
\hline $4000 \leq c z<6000$ & 20 & 0 & 0 & 0 \\
\hline $6000 \leq c z<8000$ & 15 & 1 & 1 & 0 \\
\hline$c z \geq 8000$ & 9 & 0 & 0 & 0 \\
\hline
\end{tabular}

${ }^{a}$ redshifts are corrected for Virgo infall by multiplying the corrected distances from Section VI.2.3 by $H_{0}\left(75 \mathrm{~km} \mathrm{~s}^{-1} \mathrm{Mpc}^{-1}\right)$.

(1) redshift interval (in $\mathrm{km} \mathrm{s}^{-1}$ ) of respective subsample

(2) number of sample galaxies in subsample

(3) number of galaxies in the subsample for which at least one possible companion was found

(4) total number of possible companions found for the respective subsample

(5) number of dwarf galaxies among the possible companions

\section{VI.3 Results}

\section{VI.3.1 The close environment}

\section{VI.3.1.1 Distribution of the companions and companion selection criteria}

It is conceivable that the recession velocity difference, $\Delta c z$, and the projected separation, $s_{p}$, strongly influence the dynamical response and spectrophotometric evolution of dwarf galaxies in the presence of a companion.

We assumed tidal forces to have significant dynamical influence within the range $\Delta c z<500 \mathrm{~km} \mathrm{~s}^{-1}$ and $s_{p}<0.1 \mathrm{Mpc}$. These criteria follow the estimates by CamposAguilar et al. (1993), and are comparable to those by Pustilnik et al. (2001). They are further compatible with observed values of $s_{p}$ and $\Delta c z$ for e.g. the BCD II Zw 33 and its companion II Zw 33 B, which are probably bound (Walter et al. 1997) and likely to be influenced by interaction (Méndez et al. 1999). For M51-like close pairs of galaxies, which show signs of perturbation due to interaction, similar values for $\Delta c z$ are observed (Laurikainen et al. 1998).

The distribution of our SFDG companion candidates on the $\Delta c z$ vs. $s_{p}$ plane (Figure VI.1, filled circles) peaks towards small redshift differences. This is also illustrated in Figure VI.2, which shows the frequency of the companions to be rising towards smaller $\Delta c z$. If this trend reflects a physical association between sample SFDG and companion (see Section VI.4), then our cutoff, $\Delta c z<500 \mathrm{~km} \mathrm{~s}^{-1}$, should mainly select true companions, i.e. objects likely to act as perturbers repeatedly or on longer timescales, rather than randomly passing interlopers.

Table VI.2 (first row) shows the companion statistics obtained by the latter search criteria. 


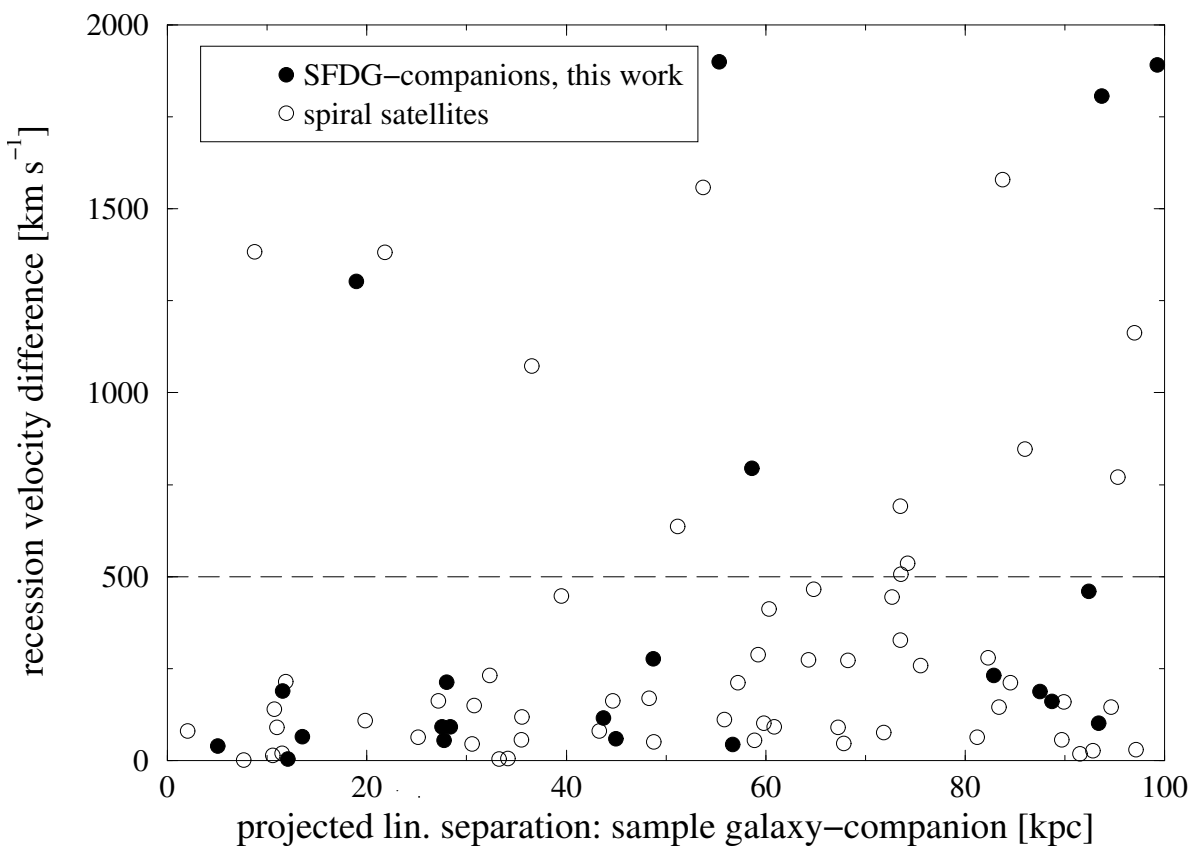

Fig. VI.1. Recession velocity difference vs. projected linear separation of the SFDGs' putative companions (filled symbols). For comparison, we show by open circles the distribution of all companions (not restricted to dwarf galaxies) we found around field spiral galaxies from the sample of Kennicutt \& Kent (1983). The dashed line represents the maximum recession velocity difference between SFDG and companion we adopt.

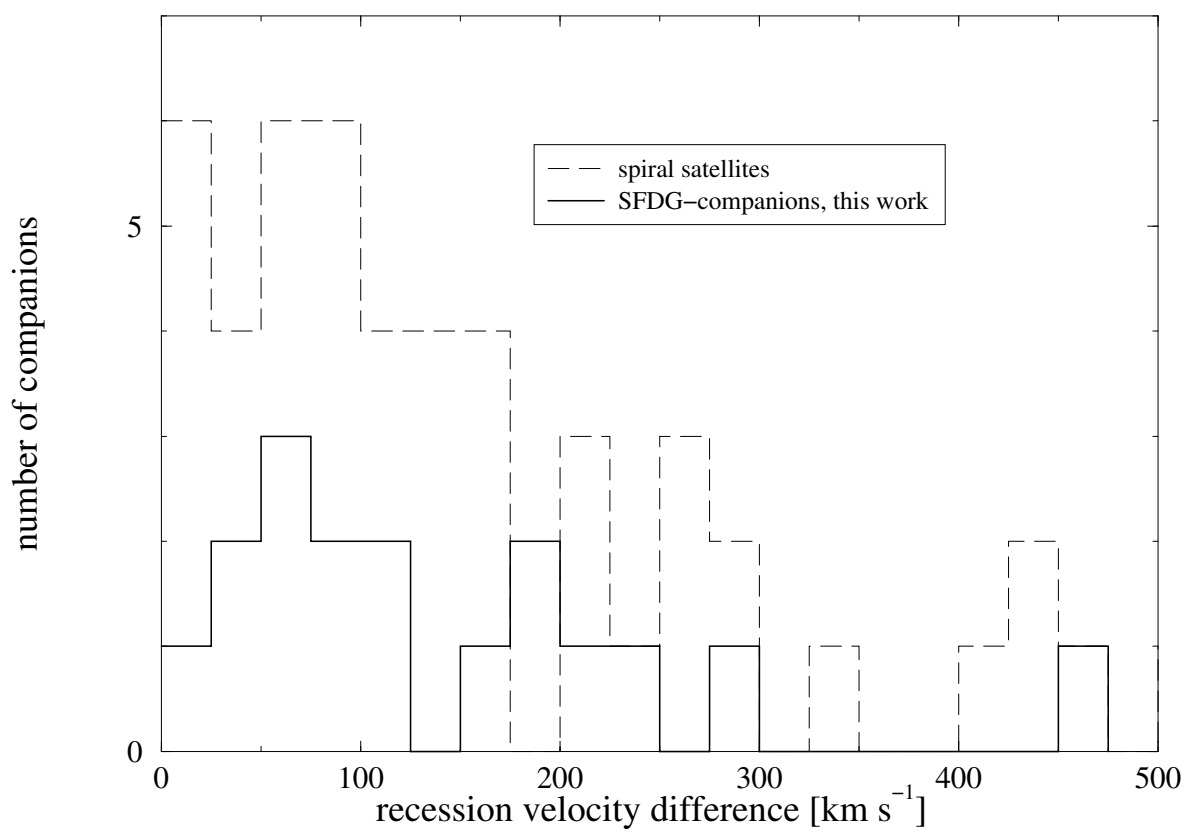

Fig. VI.2. Distribution of the recession velocity differences between the sample SFDGs and the putative companions. The dashed histogram is built from the spiral companions shown in Figure VI.1. 
We will in the following refer to the galaxies with at least one possible companion within the limits stated above as to the non-isolated sample and to those for which no companion candidates were found as to the isolated galaxies. This is for the sake of simplicity; one has to keep in mind that the isolation criteria described above are empirical, being based on assumptions and observational clues.

\section{VI.3.1.2 Companion properties; bias against faint companions}

The first row of Table VI.2 shows that the majority ( $\gtrsim 80 \%$ ) of the possible close companions are dwarf galaxies $\left(\mathrm{M}_{B}>-18 \mathrm{mag}\right)$; this is also illustrated in Figure VI.3. Their mean $B-V$ colour $(0.44 \pm 0.11 \mathrm{mag})$ is nearly equal to the average value for the sample galaxies (cf. Table VI.3), pointing to recent or ongoing SF activity. The differences between the B luminosities of each sample galaxy and its companions are shown in Figure VI.4. This distribution shows a large scatter around a median of 0.72 mag, which suggests that we tend to find primarily the brightest companion galaxies (cf. Section VI.4).

The observational bias against low-luminosity companions, i.e. against the majority of close companions of SFDGs, is also evident from Table VI.2. The rough completeness limit of the NED of $\sim 15.5 \mathrm{~B}$ mag (cf. Section VI.2), translates, by the $H_{0}$ adopted herein (Section VI.1) and assuming a pure Hubble flow, to a maximum distance of $50 \mathrm{Mpc}\left(c z \approx 3760 \mathrm{~km} \mathrm{~s}^{-1}\right)$ for a dwarf companion $\left(\mathrm{M}_{B}>-18 \mathrm{mag}\right)$ to be listed in the NED. Indeed, for $c z \geq 4000 \mathrm{~km} \mathrm{~s}^{-1}$, no dwarf companions were found. In the determination of the fraction of SFDGs which have a possible dwarf companion, one is therefore forced to stick to small redshifts. This is further demonstrated by the fact that for the subsample at $c z \leq 2000 \mathrm{~km} \mathrm{~s}^{-1}$, the fraction of non-isolated galaxies is considerably higher $(31 \%)$ than for the total sample $(16 \%)$. We will further comment on this point in Section VI.4.

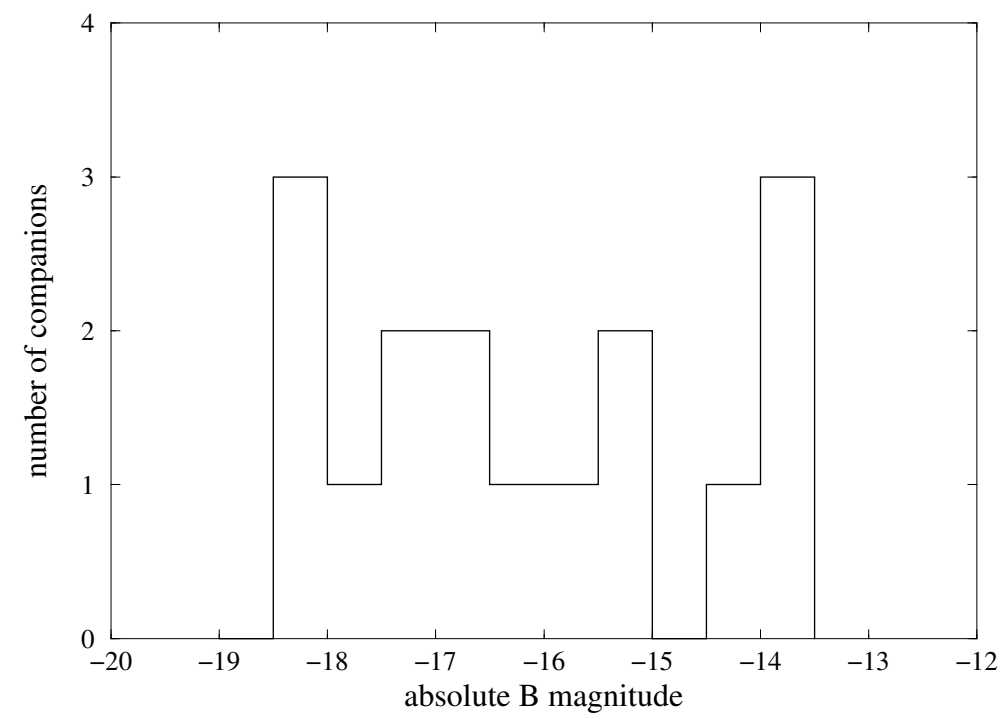

Fig. VI.3. Distribution of the B magnitudes of the SFDG companion candidates. 


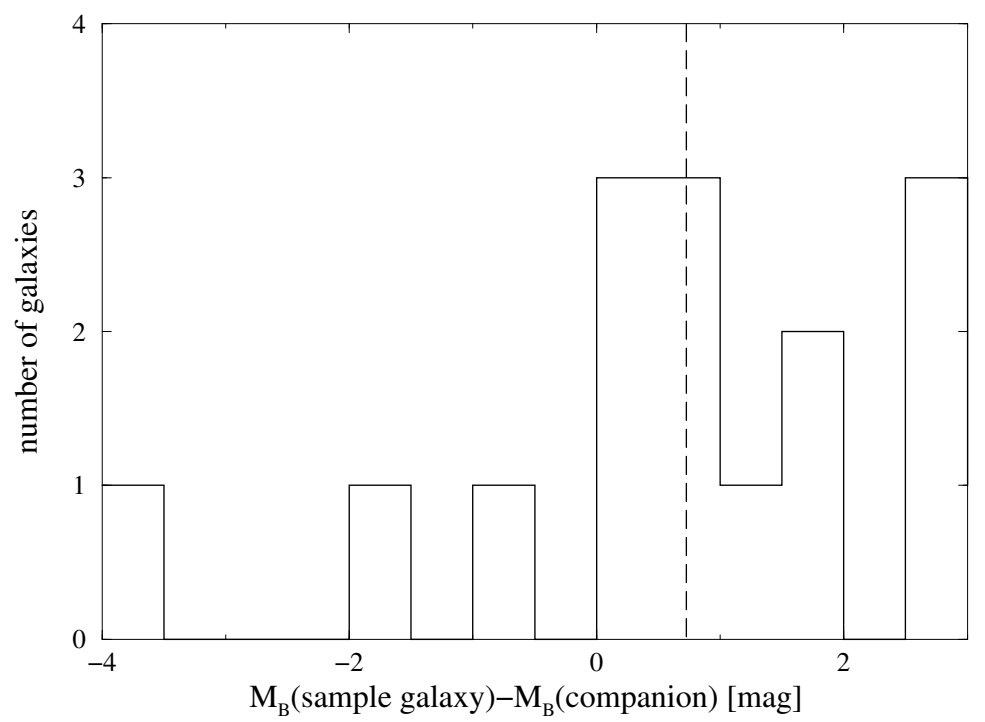

Fig. VI.4. Distribution of differences between the B luminosity of each SFDG and its found companion candidate(s). The median value of the distribution, $0.72 \mathrm{mag}$, is marked by the dashed line.

\section{VI.3.2 Star Formation and Environment}

Table VI.3. Comparison of the SF activity indicators for different subsamples.

\begin{tabular}{lccc}
\hline \hline SF indicator & isolated & non-isol. & K.-S. \\
$(1)$ & $(2)$ & $(3)$ & $(4)$ \\
\hline$E W(\mathrm{H} \beta)[\AA]$ & $68.4 \pm 15.1$ & $94.6 \pm 24.7$ & 0.45 \\
\# data points & 19 & 11 & \\
\hline$(B-V)[\mathrm{mag}]$ & $0.43 \pm 0.07$ & $0.48 \pm 0.06$ & 0.90 \\
\# data points & 27 & 13 & \\
\hline \hline
\end{tabular}

(2) Mean of the respective SF activity indicator for the 'isolated' subsample with standard deviation about the mean.

(3) Like (2), but for the 'non-isolated' subsample.

(4) Result of a Kolmogorov-Smirnov test, applied to the distribution of the respective SF activity indicator for the 'non-isolated' and 'isolated' subsamples. The probability of the hypothesis that both distributions are drawn from the same parent distribution is shown; small values suggest that the distributions are different from each other.

To assess whether the SF activity of a SFDG can be influenced by its close environment, we chose those commonly used indicators which were available for most of our sample galaxies and trace the relative strength of the SF activity on different timescales (see e.g. Schaerer 1999 for a review):

(i) The $H \beta$ equivalent width $E W(\mathrm{H} \beta)$, approximately relating the flux of Lyman continuum photons, i.e. the actual or very recent ( $\tau_{\text {burst }} \lesssim 10$ Myr) SFR, to the continuum 

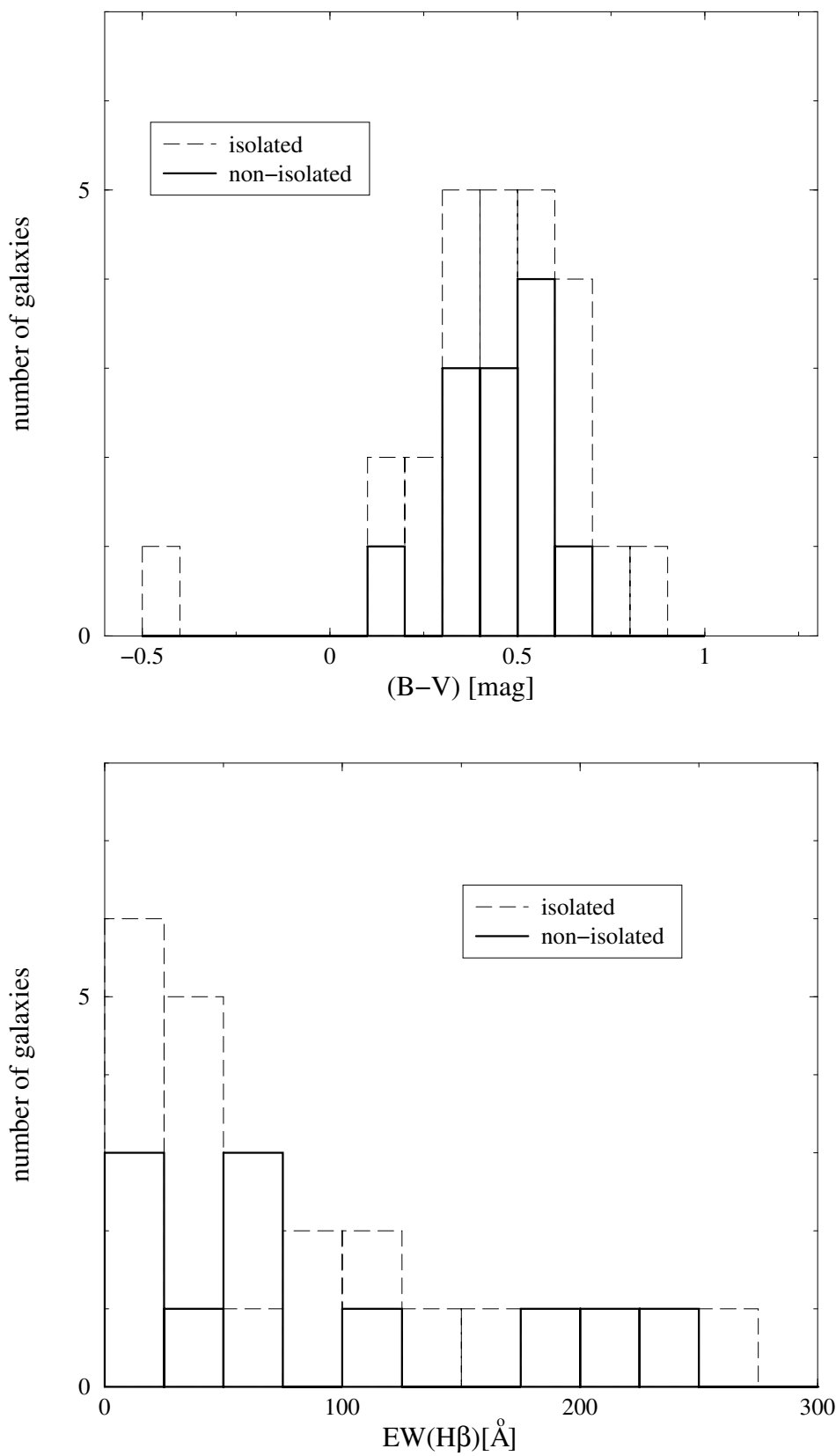

Fig. VI.5. Histograms of the top: $B-V$ colours and bottom: $\mathrm{H} \beta$ equivalent widths $E W(\mathrm{H} \beta)$ for the isolated (dashed line) and the non-isolated (solid line) subsample of SFDGs. The comparison is restricted to the sample at close distances, i.e. at redshifts $c z<2000 \mathrm{~km} \mathrm{~s}^{-1}$. 
flux in the B band, which is usually dominated by stellar emission.

(ii) The integral $B-V$ colour of the galaxies which is an indicator of the past SF activity on timescales of $\sim 10^{8} \mathrm{yr}$ (see e.g. the models by Krüger 1992 for different burst parameters). We refrain from a discussion of IRAS fluxes, originally collected and analyzed for the sample as well, as they are available for few SFDGs only, and possibly biased towards metal-rich dwarfs (see Dultzin-Hacyan et al. 1990).

In view of the bias against faint companions (Section VI.3.1.2) we stick here to the SFDGs with redshifts $c z<2000 \mathrm{~km} \mathrm{~s}^{-1}$. The above mentioned SF indicators for the isolated and non-isolated samples are compared in Figure VI.5. The corresponding statistics are listed in Table VI.3. Column (4) lists a comparison of the sample distributions by means of Kolmogorov-Smirnov tests. Both the $E W(\mathrm{H} \beta)$ and $B-V$ colours of the isolated and non-isolated subsamples are compatible with the hypothesis that they are drawn from equal parent distributions. Similarly, the sample means of $E W(\mathrm{H} \beta)$ and $B-V$ are compatible for the isolated and non-isolated subsamples within their respective sample standard deviations. Hence, from the available data, we do not confirm the higher mean $E W(\mathrm{H} \beta)$ of the 'non-isolated' sample.

\section{VI.4 Discussion}

\section{VI.4.1 Frequency, distribution and properties of the companion candidates}

The present investigation reveals that a significant fraction of SFDGs possesses possible close companions which are in almost all cases dwarf galaxies. However, the selection of the nearby SFDG subsample at $c z<2000 \mathrm{~km} \mathrm{~s}^{-1}$ does not eliminate, but merely moderates, the degree of incompleteness, which is illustrated by the distribution of the B magnitudes of the companion candidates (Figure VI.3): unlike that expected for a complete sample, it shows no increase towards fainter luminosities. In this respect, the median of the magnitude differences between a sample galaxy and its found companion candidate(s) ( $\sim 0.7$ B mag, Figure VI.4) should be interpreted as due to selection effects: the companions of SFDGs are not necessarily brighter than the SFDGs themselves, but only the brightest among the companions are listed in NED. Nevertheless, even these brightest companions qualify as dwarfs. The integrated colours of these companions are similar to those of the sample SFDGs (cf. Section VI.3.1.2), being consistent with a similar recent SF history in both sample SFDG and found companions. While this could be interpreted as a result of mutual interaction, it might again reflect the abovementioned selection effect: faint companions are most likely to be detected in emission line surveys (cf. Salzer et al. 1989b), which, of course, list preferably actively star-forming galaxies.

The quantification of the true frequency of dwarf companions suffers from the principal drawback that both the low-luminosity end of the galaxy LF and the frequency of H I companions without any optical counterpart (Taylor et al. 1994, 1995, 1996) are not yet well-constrained. Therefore, even a uniform and well-defined homogeneous completeness limit for the NED would to date not allow any meaningful extrapolation. Instead, we emphasize that the value of $\approx 30 \%$ inferred in Section VI.3.1.2 
must be considered a lower limit to the true fraction of SFDGs with close optical or purely gaseous (dwarf) companions within $\Delta c z<500 \mathrm{~km} \mathrm{~s}^{-1}$ and $s_{p}<0.1 \mathrm{Mpc}$. It must be noted that this work and the one by Pustilnik et al. (2001), performed independently and using different approaches and data, find almost equal frequencies of low-luminosity companions of SFDGs/BCDs. Furthermore, Pustilnik et al. (2001) also report evidence for a considerable number of even fainter, but yet uncatalogued companions. Altogether, this lends further support to the results by L96, who attributed the apparent extreme isolation of some BCDs to an observational bias against distant, intrinsically faint companions.

The small redshift differences between SFDGs and companions, the distribution of which rises towards smaller values, are comparable to what is found for binary galaxies (Schneider \& Salpeter 1992). In particular, a similar behaviour is reported for dwarf satellites of field spirals (Zaritsky et al. 1997). This is illustrated in Figures VI.1 and VI.2, where we plot our results of a near-object search (not restricted to dwarf galaxies) in the NED around field spirals from Kennicutt \& Kent (1983). Analogous to spiral companions, there is no evident correlation between $\Delta c z$ and $s_{p}$ for the SFDG companions. A plausible explanation of this issue was offered in the framework of a CDM scenario, taking into account a number of further dynamical arguments, by Zaritsky et al. (1997). They consider the spiral satellites to be associated with an extended massive DM halo of the mother galaxy, which strongly influences the system's dynamics, so that $\Delta c z$ is widely independent of $s_{p}$. Figure VI.1 suggests that $\Delta c z$ and $s_{p}$ are also uncorrelated for the SFDG-companion systems. If this can be corroborated by a larger dataset, then a hypothesis worth investigating for compatibility with CMD models is that SFDGs and their companions share common DM haloes of similar mass and structure.

At least, the data strongly suggest some physical association between SFDGs and their companions. In combination with our considerations on the frequency of dwarf companions, this leads to the assumption that SFDGs are frequently associated with systems of two or more dwarf galaxies. This is reminiscent of the dwarf galaxy groups L96 found in voids, and may offer important clues to dwarf galaxy formation.

\section{VI.4.2 Close dwarf companions as starburst triggers?}

The similarity of the SF tracers for the isolated and non-isolated subsamples may suggest that the observed starburst activity is regulated by solely internal processes. This hypothesis can, on the other hand, not be proven, and neither can an influence of the presence or absence of companions be disproven. Indeed, this latter hypothesis may receive some support from the marginal trend in $E W(\mathrm{H} \beta)$ that the data shows. The large scatter of the analyzed SF diagnostics is not surprising, for a number of reasons:

(i) The severe incompleteness of the available data aggravates the separation of galaxies with and without close companions.

(ii) Balmer emission lines, i.e. Lyman continuum tracers, correspond to short-term variations in the SFR. The latter are particularly strong in SFDGs, where SF generally 
occurs in short bursts of $\lesssim 10^{7} \mathrm{yr}$ (Elmegreen et al. 1996). Considerable variations with time also occur for the $B-V$ colours after the onset of a starburst (see e.g. Krüger 1992). Whatever triggers a starburst, the time window during which strongly enhanced SF indicators are observable is narrow. In addition, such SFR tracers also depend on evolutionary parameters - metallicity, age and stellar continuum contribution - of the SFDG itself.

The hypothesis of interaction-induced starburst activity in SFDGs might be more reliably assessed when a large sample of SFDGs with sufficiently deeply observed environments is available. However, the abovementioned intrinsic sources of scatter certainly limit the usefulness of a comparison that is restricted to integral SFR tracers.

This research emphasizes the need for a systematic effort on the part of theory to advance the understanding of conditions required for inducing and sustaining starburst activity in dwarf galaxies, with particular emphasis on dwarf galaxy interactions.

\section{VI.5 Summary and Conclusions}

We have searched the close environment of a sample of 98 star-forming dwarf galaxies (SFDGs) in field and low-density environments, taken from different catalogues, for possible companion galaxies. To supplement previous work, which had mostly dealt with luminous companions, a subsample of nearby SFDGs was chosen to moderate the effect of observational bias against low luminosity companions. Distances were obtained from the recession velocity of the sample galaxies assuming a pure Hubble flow and have been corrected for Virgocentric infall. Using the Nasa Extragalactic Database (NED) as a search catalogue, objects with a projected linear separation of $<100 \mathrm{kpc}$ and a recession velocity difference $<2000 \mathrm{~km} \mathrm{~s}^{-1}$ were catalogued as possible companions of a SFDG. Most of them differ in their recession velocity by $<500 \mathrm{~km} \mathrm{~s}^{-1}$ from the sample SFDGs, which, along with a number of other considerations, led us to adopt this value as a limit to identify possible companions. For both SFDGs and companion candidates, spectrophotometric data were compiled from the NED and a number of literature sources.

We studied the frequency, redshift difference distribution, and photometric properties of companion candidates, as well as their possible influence on the star formation (SF) activity of the sample SFDGs. Our results can be summarized as follows:

(i) A substantial fraction of SFDGs possess companion galaxies within its close environment. The overwhelming majority of these companions are dwarfs $\left(\mathrm{M}_{\mathrm{B}}>-18\right)$, which renders their detection at large distances difficult. The fraction of SFDGs for which we detect dwarf companion candidates, $\approx 30 \%$, must be considered a lower limit to the true value, given the increasing incompleteness of the available data set for systems fainter than $m_{B} \sim 15.5 \mathrm{mag}$. A meaningful extrapolation to fainter magnitudes is precluded by the poorly constrained frequency of H I companions with no optical counterpart, and by the uncertain faint end of the galaxy luminosity function.

(ii) The recession velocity differences between SFDGs and identified companions amount typically to $\$ 250 \mathrm{~km} \mathrm{~s}^{-1}$. Their frequency rises towards lower differences. 
This is similarly reported for dwarf companions of spirals, and suggests that the SFDG companions are physically associated with the sample galaxies.

(iii) Both the $B-V$ colours and the $\mathrm{H} \beta$ equivalent widths appear compatible for objects with and without a possible perturber. The significance level of the data is too low to prove the hypothesis of a solely internal regulation of the SF activity, whereas external influence cannot be disproven either. The considerable intrinsic scatter inherent to statistical studies based on the abovementioned SF diagnostics, together with the insufficiently complete companion search databases, blurs the picture.

The identification of companions in the close environment of SFDGs readdresses the question of whether gravitational interactions are partly responsible for the onset of SF activity in gas-rich dwarfs. Whereas interaction-induced SF activity was dismissed for most SFDGs due to the absence of luminous companions, the presence of faint dwarfs in the close vicinity of SFDGs provides again support to this hypothesis. While deep radio and spectrophotometric surveys may yield more conclusive answers, it will equally be important to theoretically model the effects of interactions on dwarf galaxies.

Acknowledgement. KGN gratefully acknowledges financial support from the German Research Foundation (DFG) grant FR325/50-1, and from the IAC Summer Research Programme 1998. This study was partly financed by the Spanish DGES (Dirección General de Enseñanza Superior) (grant PB97-0158). PP received support from Deutsche Agentur für Raumfahrtangelegenheiten (DARA) GmbH grant 50 OR 9907 7. This Research has made use of the NASA/IPAC Extragalactic Database (NED) which is operated by the Jet Propulsion Laboratory, CALTECH, under contract with the National Aeronautic and Space Administration. We have made use of the Lyon-Meudon Extragalactic Database (LEDA) supplied by the LEDA team at the CRALObservatoire de Lyon (France). We thank S.A. Pustilnik and A. Kniazev for fruitful discussions and helpful comments on this paper. KGN wishes to thank L.M. Cairós, J. Iglesias, J. Vílchez and the IAC staff for their hospitality. We thank the referee, Dr. Metcalfe, for his helpful comments.

\section{References}

Cairós, L.M., Vílchez, J.M. 1998, in: The Magellanic Clouds and other Dwarf Galaxies, eds. Richtler, T., Braun, J.M., Shaker Verlag, p.235

Cairós, L.M., Vílchez, J.M., González-Pérez, J.N., et al. 2000, ApJ, in press

Campos-Aguilar, A., Moles, M. 1991, A\&A, 241, 358

Campos-Aguilar, A., Moles, M., Masegosa, J. 1993, AJ, 106, 1784

Davies, J.I. \& Phillipps, S. 1988, MNRAS, 23, 553

Doublier, V., Caulet, A., Comte, G., 1999 A\&AS, 138, 213

Dultzin-Hacyan, D., Masegosa, J., Moles, M. 1990, A\&A, 238, 28

Elmegreen, B.G., Elmegreen, D.M., Salzer, J.J., Mann, H. 1996, ApJ, 467, 579

French, H.B. 1980, ApJ, 240, 41

Garcia, A.M. 1993, A\&AS, 100, 47

Gerola, H., Seiden, P., Schulmann, L. 1980, ApJ, 242, 517

Grogin, N.A. \& Geller, M.J. 2000, AJ, 119, 32

Guzmán, R., Jangren, A., Koo, D.C., Bershady, M.A., Simard, L. 1998, ApJ, 495, L13 
Hashimoto, Y., Oemler, A., Lin, H., Tucker, D.L. 1998, ApJ, 499, 589

Hensler, G., Rieschick, A., Köppen, J. 1999, in "The Evolution of Galaxies on Cosmological Timescales", J. Beckman \& T. Mahoney (eds.), Ap\&SS, in press (astro-ph/9908242)

Kennicutt, R.C. \& Kent, S.M. 1983, AJ, 88, 1094

Kennicutt, R.C., Roettiger, K.A., Keel, W.C., van der Hulst, J.M., Hummel, E. 1987, AJ, 93, 1011

Kraan-Korteweg, R.C. 1986, A\&AS, 66, 255

Krüger, H. 1992, PhD Thesis, Universitäts-Sternwarte Göttingen

Laurikainen, E., Salo, H., Aparicio, A. 1998, A\&AS, 129, 517

Lee, J.C., Salzer, J.J., Law, D.A., Rosenberg, J.L. 2000, ApJ, 536, 606

Lindner, U., Einasto, M., Einasto, J., Freudling, W., Fricke, K., Lipovetsky, V., Pustilnik, S., Izotov, Y., Richter, G. 1996, A\&A, 314, 1 (L96)

Loveday, J., Tresse, L., Maddox, S. 1999, MNRAS, 310, 281

Marinoni, C., Monaco, P., Giuricin, G., Costantini, B. 1998, ApJ, 505, 484

Marlowe, A.T., Meurer, G.R., Heckman, T.M. 1999, ApJ, 522, 183

McAlpine, G.M., Lewis, D.W., Smith, S.B. 1977a, ApJS, 35, 203

McAlpine, G.M., Smith, S.B., Lewis, D.W. 1977b, ApJS, 34, 95

McAlpine, G.M., Smith, S.B., Lewis, D.W. 1977c, ApJS, 35, 197

McAlpine, G.M. \& Lewis, D.W. 1978, ApJS, 36, 587

McAlpine, G.M. \& Williams, G.A. 1981, ApJS, 36, 587

Méndez, D.I., Cairós, L.M., Esteban, C., Vílchez, J.M. 1999, AJ, 117, 1688

Méndez, D.I. \& Esteban, C. 1999, AJ, 118, 2723

Noeske, K.G. 1999, Diploma Thesis, Universitäts-Sternwarte Göttingen

Noeske, K.G., Cairós, L.M., Papaderos, P., Vílchez, J.M., Fricke, K.J. 1998, in ”The Evolution of Galaxies on Cosmological Timescales", J. Beckman \& T. Mahoney (eds.), Ap\&SS, in press

Noeske, K.G., Papaderos, P., Fricke, K.J., Thuan, T.X. 1998, in: The Magellanic Clouds and other Dwarf Galaxies, eds. Richtler T., Braun J.M., Shaker Verlag, p.279

Odewahn, S.C. 1994, AJ, 107, 1320

Papaderos, P., Loose, H.-H., Fricke, K.J., Thuan, T.X. 1996, A\&A, 314, 59

Pilyugin, L. 2000, A\&A, 354, 874

Popescu, C.C., Hopp, U., Rosa, R. 1999, A\&A, 350, 414

Pustilnik, S.A., Kniazev, A.Y., Ugryumov, A.V. 1997, IAUJD, 2E, 60

Pustilnik, S.A., Kniazev, A.Y., Ugryumov, A.V. 2001, A\&A, 373, 24

Pustilnik, S.A., Ugryumov, A.V., Lipovetsky, V.A., Thuan, T.X., Guseva, N. 1995, ApJ, 443, 499

Rosenberg, J.L. \& Salzer, J.J. 1994, AJ, 108, 1557

Salzer, J.J. 1989, ApJ, 347, 152

Salzer, J.J., McAlpine, G.M., Boroson, T.A. 1989a, ApJS, 70, 447

Salzer, J.J., McAlpine, G.M., Boroson, T.A. 1989b, ApJS, 70, 479

Salzer, J.J \& Norton, S.A. 1998, IAUC, 171

Savage, B.D. \& Mathis, J.S. 1979, ARA\&A, 17, 73

Schaerer, D. 1999, in "Building the Galaxies: from the Primordial Universe to the Present", XIXth Moriond astrophysics meeting, F. Hammer et al. (eds.), World Scientific, p.389

Schneider, S.E. \& Salpeter, E.E. 1992, ApJ, 385, 32

Shull, J.M., Stocke, J.T., Penton, S. 1996, AJ, 111, 72

Taylor, C.L., Brinks, E., Podge, R.W., Skillman, E.D. 1994, AJ, 107, 971

Taylor, C.L., Brinks, E., Grashius, R.M., Skillman, E.D. 1995, ApJS, 102, 189

Taylor, C.L., Thomas, D.L., Brinks, E., Skillman, E.D. 1996, ApJS, 107, 143 
Telles, E. \& Terlevich, R. 1995, MNRAS, 275, 1 (TT95)

Telles, E. \& Maddox, S. 1999, MNRAS, 311, 307

Terlevich, R., Melnick, J., Masegosa, J., Moles, M., Copetti, M.V.F. 1991, A\&AS, 91, 885 (SCHG)

Thuan, T.X. 1985, ApJ, 299, 881

Thuan, T.X. 1991, in: Massive Stars in Starbursts", eds. C. Leitherer, N.R. Walborn, T.M. Heckman, C.A. Norman, Cambridge University Press, p.183

Thuan, T.X. \& Martin, G. 1981, ApJ, 247, 823

Tully, R.B. 1988, Nearby Galaxies Catalog, Cambridge University Press

Tully, R.B. \& Shaya, E.J. 1984, ApJ, 281, 31

Vennik, J., Hopp, U., Popescu, C.C. 2000, A\&AS, 142, 399

Vílchez, J.M. 1995, AJ, 110, 1090

Vílchez, J.M. 1997, RMxAA (Serie de Conferencias), 6, 30

Walter, F., Brinks, E., Duric, N., Klein, U. 1997, AJ, 113, 2031

Wilcots, E.M., Lehman, C., Miller, B. 1996, AJ, 111, 1575

Zaritsky, D., Smith, R., Frenk, C., White, S.D.M. 1997, ApJ, 478, 39

Zwicky, F. 1961, Catalogue of Galaxies and Clusters of Galaxies (CGCG) 



\section{Lebenslauf}

Ich wurde am 23. März 1973 in Darmstadt als Sohn von Olaf Rüdiger Noeske und Isolde Luise Noeske (geb. Lein) geboren. Ich bin deutscher Staatsbürger.

Von 1979 bis 1983 besuchte ich die Grundschule Ulrichstein. Während der darauffolgenden zwei Jahre war ich Schüler der Förderstufe der Eichbergschule Lauterbach/Hessen. Von 1985 an besuchte ich das Alexander-von-Humboldt Gymnasium in Lauterbach, wo ich im Sommer 1992 mein Abitur erwarb.

Von 1992 bis 1993 leistete ich Zivildienst als Rettungssanitäter beim Rettungsdienst des Deutschen Roten Kreuzes in Lauterbach.

Im Oktober 1993 immatrikulierte ich mich an der Georg-August-Universität Göttingen im Fachbereich Physik. Im Oktober 1995 legte ich die Diplomvorprüfung ab. 1997 begann ich mit der Anfertigung meiner Diplomarbeit an der Universitäts-Sternwarte Göttingen unter der Betreuung von Prof. Dr. Klaus Fricke, die ich im Februar 1999 mit der Diplomprüfung abschloss.

Meine Promotion zum Thema "Optical and Near Infrared studies of the photometric structure and starburst activity of Blue Compact Dwarf Galaxies" begann ich im März 1999 an der Universitäts-Sternwarte Göttingen, ebenfalls unter der Betreuung von Prof. Dr. Fricke.

Während meines Hauptstudiums war ich wiederholt als studentische Hilfskraft beschäftigt, von 1995-1997 mehrmals am Max-Plack-Institut für Strömungsforschung in Göttingen, und von 1997-1999 an der Universitäts-Sternwarte Göttingen. 1999 war ich teils als wissenschaftliche Hilfskraft, teils als wissenschaftlicher Mitarbeiter im FORS-Projekt, an der Universitäts-Sternwarte angestellt. In den Jahren 2000-2002 war ich durchgehend als wissenschaftlicher Mitarbeiter im DFG-Projekt "Optische und Nahinfrarot-Beobachtungen zur Struktur und Entwicklung Blauer Kompakter Zwerggalaxien" beschäftigt.

1998 arbeitete ich drei Monate lang als Stipendiat des "IAC Summer Research Program" am Instituto de Astrofísica de Canarias, Teneriffa/Spanien. Von 1999 bis 2003 verbrachte ich mehrere Gastaufenthalte am IAC/Teneriffa und am IAA/Granada, sowie kürzere Aufenthalte an verschiedenen Instituten in den USA (University of Florida/Gainesville, University of Pittsburgh, University of Virginia/Charlottesville, Lick Observatory/Santa Cruz, CALTECH/Pasadena). Im selben Zeitraum unternahm ich mehrere Beobachtungsreisen zu den Calar Alto- und Teide-Observatorien in Spanien, sowie zur Europäischen Südsternwarte nach Chile. 